\title{
NUMERICAL SIMULATION OF LASER FUSION PLASMAS USING A REDUCED DESCRIPTION FORMALISM
}

Rickey James Faehl

(Ph.D. Thesis)

jecember 1976

Prepared for U.S. Energy Research \& Development

Administration under contract No. W-7405-Eng-48

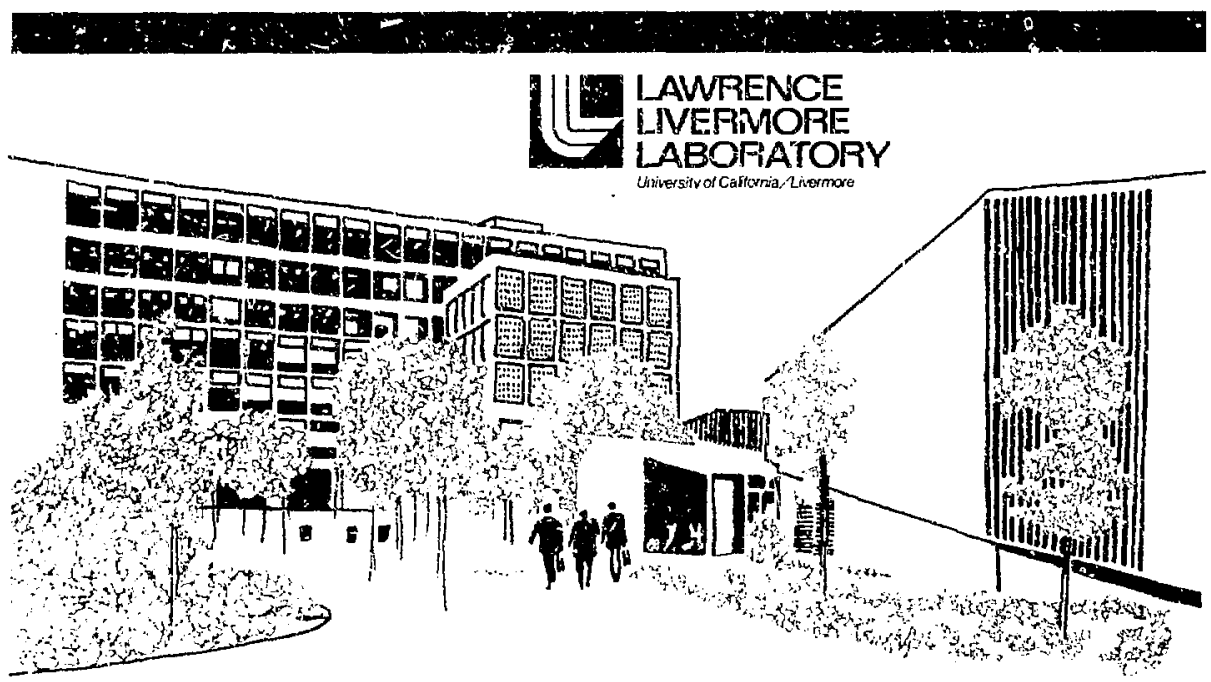




\section{NaTIC:}

This repust was prepared as an accotenl of wolk sponsored by the United Stales (iutermenth. Neither the United States nor the United Siales Entergy Rescarch \& Deveiopment Administation. nor any of theis employees, nur any of their cuntracturs, subsontractuss. oi theit emrloyees, makes any wartanty. express or implied, of assumes suy lega! liabidity or :esponsibility for the accuracy, completertess or uselulacse of ally information, apparatus, product or process diselosed, or represents that its use would not inftinge privately-owned rights.

\section{NOTICE}

Reference to a company or product num tous nut imply approval or secommendation of the produst by the Universiiy of Calitornia or the U.S. Energy Reseasth \& Development Adninistration to the exclusion of others that may be suitable.

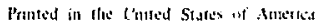

Avislahk irm!t

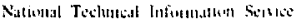

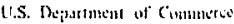

S2S5 Purt Ruyal Ruad

Springintld. VA 221G1

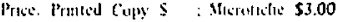

\begin{tabular}{|c|c|c|c|c|c|}
\hline Page & Pange & $\begin{array}{c}\text { Domestic } \\
\text { Price }\end{array}$ & Page & fange & $\begin{array}{c}\text { Domestic } \\
\text { Price }\end{array}$ \\
\hline 001 & 025 & $S .3 .50$ & $\$ 26$ & 3.50 & 10.00 \\
\hline 036 & 050 & 4.00 & 351 & 5 & 10.50 \\
\hline 051 & 075 & \pm .50 & $\$ 76$ & +100 & 10.75 \\
\hline 076 & 100 & 5,00 & 401 & $+\therefore$ & $1: 00$ \\
\hline 101 & 125 & 5.50 & 436 & 150 & 11.75 \\
\hline 120. & 150 & 6.00 & 451 & 475 & 12.00 \\
\hline$|5|$ & -175 & 6.75 & 476 & soo & 12.50 \\
\hline 76 & 200 & 7.50 & sol & 525 & $1: 75$ \\
\hline 201 & -225 & 7.35 & 526 & 550 & 1.3 .20 \\
\hline 226 & -250 & $\$ .00$ & آis & 375 & 13.50 \\
\hline 251 & 275 & 9.00 & $57 \mathrm{f}$ & $(300)$ & 1.75 \\
\hline 276 & 300 & 9.25 & 601 & a. & $*$ \\
\hline 303 & 325 & 9.75 & & & \\
\hline
\end{tabular}

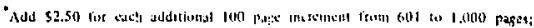

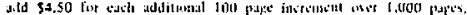




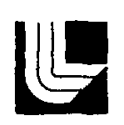

\title{
I_AWRENCE I.IVERMORE I_ABORATORY
}

Unwersityol Catoma Livermure Callorna 94550

\section{NUMERICAL SIMULATION OF LASER FUSION PLASMAS USING A REDUCED DESCRIPTION FORMALISM}

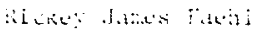

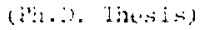 \\ i)ewher I4,"in
}

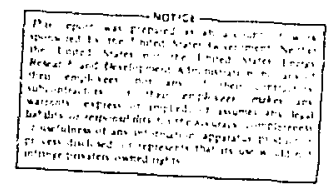




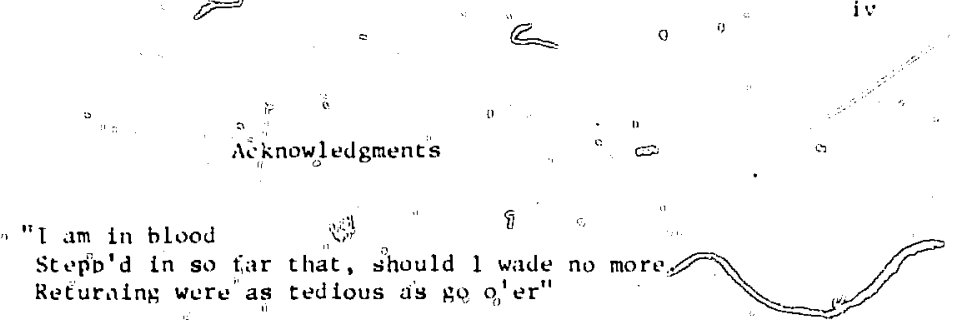

Macbeth Act III, Scenc IV

It is not false modesty to state that this study could not have bean completed without the aid andosupport of mathy people. 1 wish to acknowledge my deepest appreciation here to the Tull's, Estabrook's, ialeo's, liruer's, and Thumson's, without whose frtendship and hospitdity I should have had a mush more trying time commuting between

Livermore and Albuquerque: Kent Estabrook, Ernest Valeo, Jeff Thomson, Bruce" Langdon, and, Barbara Lasinski have also provided, aside trona a spare bed on occasion, valuable discussions, ctiticism, and/or support for this project.

This largely numerical study would have been impossible without the extended computational support I received from $\mathrm{X}$-Croup, both as an Air Force Resecrch Assuciate at livermore, and later when" 1 was assigned to A.F.W.I. L hope that at least some of the results of this" work may prove useful to the laser fusion prontam.

The difficult job of preparing this manuscript has been superbly accomflished by Mrs. Fsther Caster.

Norman koderick deserves a special thanks." As my supervisor and friend "in the Simulation Branch, his" encuuragement to finish what I came to regardas a monstrosity was truly invaliable.

John Decroot is my first mestor it p Pasma physics. If I have 

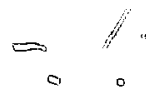

learned anything of the simulation of plasmas, I cmust credit John for redirecting my experamentalist inclinations toward that end. He taught me not imply a collection of sundry techniques, buf the inituitics for model1ng. DThS, I regard as much more subtle and difficult to transmt. Finally, Dr. W1lilam Kruer has takèn "me from a "callow graduate student to, physiciat. My understand1 $\hat{f}_{\mathrm{e}}$ of the phyeical world and appreciation of 1ta mystery and excitement have been immeasurably enhanced by him. He has taught we to be surprised, to expect the unexpected. "I Ereely acknowledge profound gratitude for this. And throughout, Allyson has hot contributed in any direct way

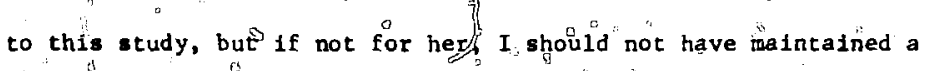
semblance of sanity as long as I have.

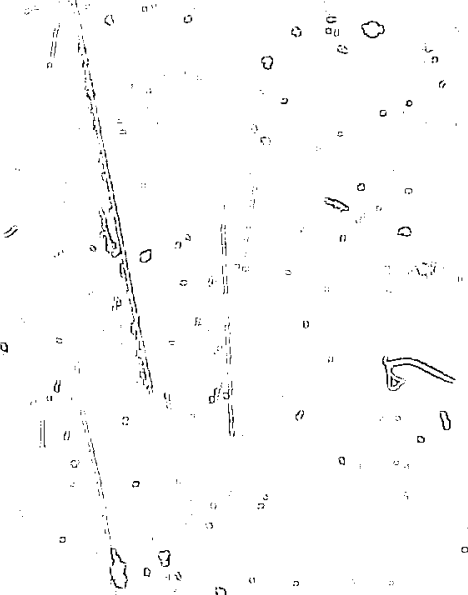




\section{Táble of Concents}

abstract.................................. Tii

Acknowledgments............................. iv

Table of Contents. $\ldots \ldots \ldots \ldots \ldots \ldots \ldots \ldots \ldots \ldots \ldots \ldots \ldots \ldots$

Chapter 1: Introduction. .......................

Chapter 2: The Reduced Description of Laser Plagna...........

I: Description of the Reduced Description Formal1sm: (RDF). 9

II: Factors Affecting the Swelling of Electromagnetic

Waves Neat the Crftical Density:................ 13

${ }_{B}$ III : Self-Steepening of Density Profifes dear the Critical

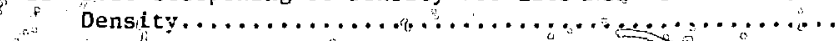

Chapter 3: Stimulated Brillouin Scatteting and Mode Cuupling: - 0 . Nonlinear Evolution of a Parametric Instability....

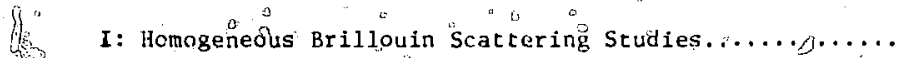

II Inhomogeneous Brillouing Scatteing studies......... 51

"II: Applications of Inhomogeneous Calculations to a

we, \#

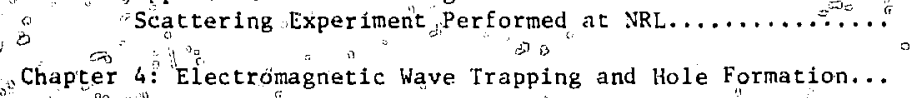

"I: Wave Trapping/Hole Formation in" a Homogeneous Plasima..

II: "Wave Trapping/Hole Formation in Gan Inthomogenous

III : Discussion of Wave/7rapping/Hole Formation Results....:

II: Direct Intensity Dependence in Collisional Absorption. : 136 
oIII: Enhangement of Laser Absorption wth" Short Wavelength Ion

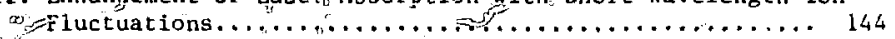

Chapter 6: Conclusions and Recomendations............... 170

Appendix A: The Reduced Description Equations $\ldots \ldots \ldots \ldots$

Appendix B': Linear Theory of the "Stimulated Brillouin/

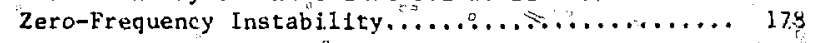

Appendx c: Derivation of Coupled Wave Equations From the

Reduced Description Equations................ 193

Appendix D: Derfvarton of the Ion Denstty Fluctuation

Induced Dissipation Rate.................... 195

Appendix E: Application of Inhomogeneous Calculations to a

Scaccering experiment Performed at NRL..., . $\ldots, 197$

Appendix F: Derivation of Jacobean Wave Function........... 205

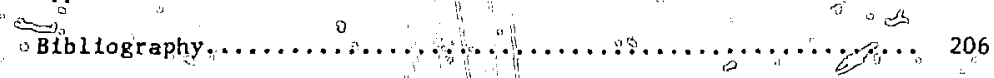

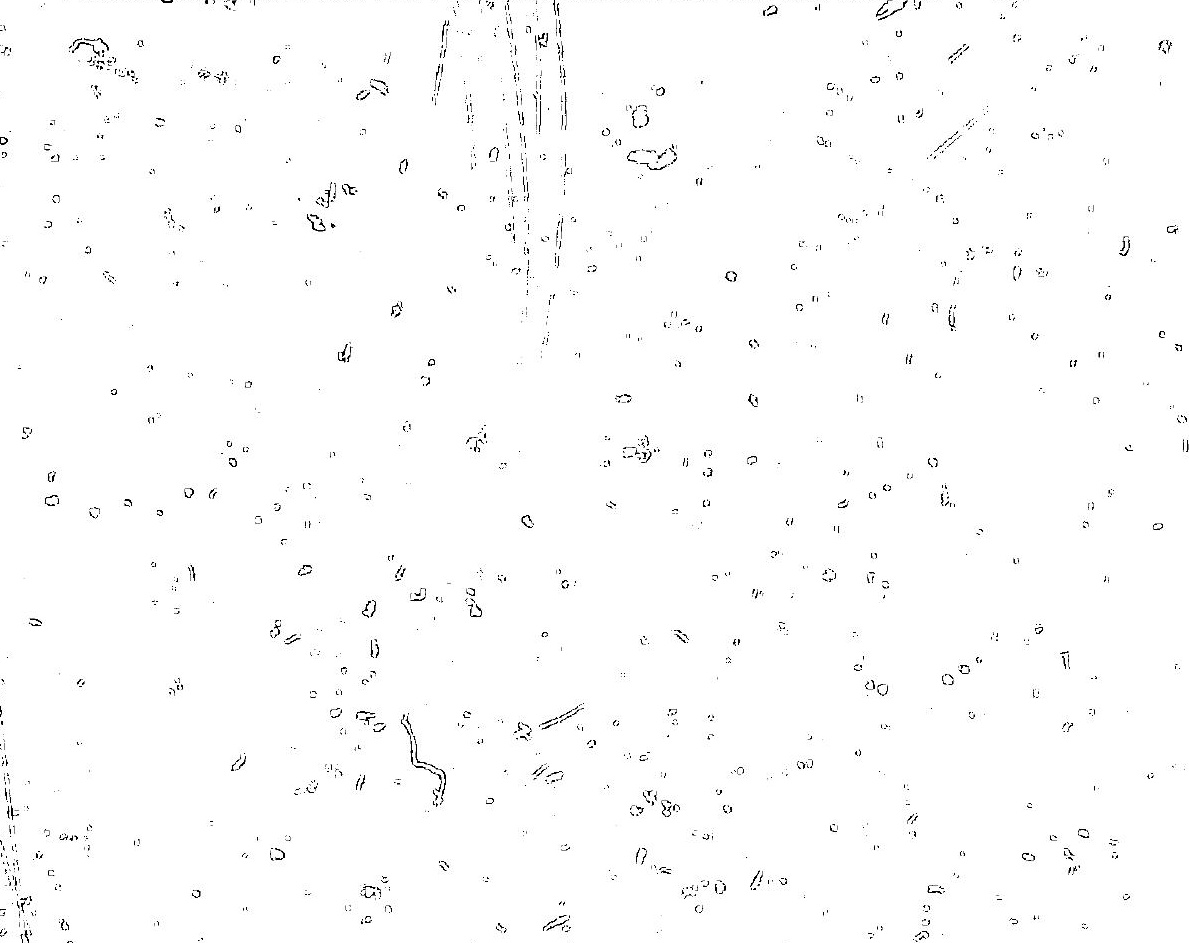



but the nere fact that such features nake an cbservable contribution suggests that the simulations may have uissed other interesting Thenomena.

One reason particle simulations may not licive detected such effects is that, while they represent a close approximation to actual coilisionless plasmas, they have certain profound limitations. Anong these are the high level of numerical noise and the slow speed. These lead to a tendency to follow systems which are too small for: too shori a time. In conseculence, an effect sesn in a careful simulation is probility real, but it is not necessariny the one which will persist Long tnough to register on an $\mathrm{ex}_{\mathrm{p}}=\mathrm{rimentalist} \mathrm{s}$ meter.

A flexíble numerical tool, involving a reduced description of the plasma, was developed to complement the simulation technique and to circtmvent the above restrictions. The tro-fluid plasma equations together with Maxwell's equations vere found to provide a powerful tool for the description of laser-illuminated plasmas, inciuding wese propagation, hydrodynamics, laser absorption, and scattering instability evolution. The dramatically reduced calculation speed facilitated long duration, quiet studies of the self consistent oneaimensional laser/plasma interaction problen.

A reduced description of the plasma is discussed in Chapter 2. Stimulated reflection coefficients are calculated with this method for a linear density grafient plasma, and these are compared with corresponding simulation values. The good agreement, up to levels then trapping dominates, confimed the validity of this approach. 
The inverse brensstrahlung absorpticn coefficient is a crucial paramet:r in laser fusion schemes, yet it is rot contained within the particle simulation technique. It was a trivial factor to include in the reduced description. Comparisons with theory veritsed the calibration. Another linear problem concerns the magnitude of the iaser field in the vicinity of the critical surface. Analytic ther: gives a standing Airy patcern in a linear density pratiant. The exact steady pattern calculated here was compared with that solution, and was also done for nonlinear density gratients, varjous liagnitudes of collisional absorption, and finite laser bandwidth. The resulting intensity potterns were related to the Airy value through approximate analytic mocels or simple heuristic expressions. Firally, Kidje: predicted that the zeroth oruer ponderomotive force from the standing laser wave near the critical density would steepen the densfty profiie. This was numerically confirmed.

In Chapter 3 , the reduced plasma description was used to study the post-saturation evolution of the scattering instabilities. A common feature of any laser plasma scattering experiants is the enhancea width of the scattered spectrum. The stimulated scattering should, however, narrow the spectrum, at least within the linear theory framework. Homogeneous, periodic calculations wore conducted first, to eliminate the complication of end-effects. It was found convenient to spatially Fourier analyze the reduced equatuons first, and to follow the evolution of the spectrum directly. Two distinct features were found, harmonlc generation driven by large amplitude 
acoustic waves, and repeated scattering. This latter has been observed experimentally in gases and liquids, so there are grounds for expecting tt in long scale length plasmas. The implied long propagation paths are inconsistent with backscattering, bur may be plausible for sidescattering. Finite system calculations verified the existence of these scattering mechanism: in locally hitrogenesws systems, but they were severely weakened 1 . ir:onogeneous ones. The absence of spectral btoadening in the backscatter direction, for scale lengths tpical of recent experiments, while broadening is still occurring in a siaescatter mode, is consistent with the neasurements reported iy an NRL group.

Chapter 4 dea]s with the trapping of intense electromagnetic signals in seIf-consistent longitudinal density fluctuations. This phenomenon was apparently First suggested by Gurevich and Karpman, though not verified. Paricle simulations and reduce, description calculations show the existence of such structures near, but below, the critical density when the field is above a threshold value. The spectral evolution of the field and the density perturbations were followed in the fluid calculatiois. The final k-spectrum has a nearly exponential form, reminiscent of a solitary wave. Periodic calculations show that mode coupling leads toward this resuit as weil. End effects play a role in the establishment of the stable structures, but the effect depends primarily on the losal value of the rield, Formation is not greatly inhibited by gradient effects, as Iong as the density scale length is significantly greater than the width of the hole. Bandwidth also has little effect on either 
fomation or stability of the structures. The one-dimensiona] cajeulations are finally discussod witl respect to uther, higher dimensional calculations performed by Valeo and Estabrook and by ctheas. In Chapter 5, various absorption related topics are stucied with the reduced description. The first section deals with reductions in classical absorption induced by density modifications. Stinialated Brillouin scattering as expected is found to have a highly deleterious effect on collisional absorption. Self-steepening rends to be dominated by SBS as an absorption reducer, eycept in very sten gradien: profiles. In that case, steepening is found to reduce absorption efficiency by factors on the order of fifty percent. In the next section, the intensity dependence of the inverse bremsstrahlung crosesection, which is usually neglected, is treated. For intensities comparable to those being considered for stimularej processes, a standing wave pattern in moderate length system is found to induce reduction factors of 2-3 in total abscrption. The effect depends pri marily on the local magnitude of the field, so a standing wave is nore effective in inhioiting absorption than a traveling wave. rinally, in the last section, a new absorption mecharism, based on scattering oscillating electrons from finte density fluctuations, is discussed. Calculatiuns siow that this mechanism has the desirable properties that it is relatively insensitive to censity modulations near the critical density, extends over a wide density range, and produces reasonably soft heated electron distributions. Various sources for the density fluctuarions are discussed. 
The conclusions of this study are sumerized in the last chapter. The relevance of the results to laser fusion experimants is briefly discussed, and extensions of this work to future "ludies are presented. 


\section{CHAPTER 2}

The Reduced Description Model of Laser Plasma

There are many ways to describe a plasma syster. The complete classical description consists of specifying the position and monentur of each particle and the fields at each position. A plasma is rot meaningful, however, un] ess the physical dimensions are greatcr than several Debye lengths. Such a volume contains too many particles to permit practical applications of a complete description. Plasma sinulation techniques ${ }^{1,2}$ follow the evolution of a system by moving quasiparticles in their self-consistent fields. Tr.e fields are s?liconsistert because their sources, density and current density are just the local averages over the positions ard velocities of tire particles. This procedure is virtually ecuivalent to solving Liouville's equation for a given set of initial conitions. The technique closely models the behavior of actual collisionless plasmas, but: it seems improbable that all problems of interest require such detailed information. In particular, the high noise levels and expense involved with such techniques iadicate that ar: accurate, reduced description of the plasma would have many applications. One such reduced description, which couples the high frequency field equations with the two-fluid plasma equations, has baen derived here. The body of this work is devoter to deternining the extent to which this description can be applied to laser plasma 
systems. In this chapcer, the formatism is first described, and chert Eested aginst a variety of conditions for which kwow solutions, or approximace soluticns, exist. 
SECTION I

Description of the Reduced Description Formalist? (RDF)

Ti.e collective processes which characterize the fast time scale bebavior of a plasma require a wave description. The reduced equations must. therefore contaln at least enough information to follow waves. The first two fluld equations of a species with charge, $q$, and mass, m, $i$ e., for particle density, $n$, and flux, $\bar{\Gamma}=n \vec{v}$,

$$
\begin{aligned}
& \frac{\partial n}{\partial t}=-\nabla \cdot \bar{T} \\
& \frac{\partial \bar{\Gamma}}{\partial t}=-\nabla\left(\frac{\Gamma^{2}}{n}\right)+\frac{q}{m} \bar{E}+\frac{q \bar{\Gamma}}{m c} \times(\nabla \times A)-\frac{1}{m} \nabla \cdot \vec{p}-v \bar{\Gamma}
\end{aligned}
$$

can easily be shown to yleld such a wave erjation. In these equations $\bar{E}=-\nabla \phi-\frac{1}{c} \frac{\partial \vec{A}}{\partial t}$ is the longitudinal electric field, $\bar{A}$ is the vector potentlaI and $v$ a heuristic damping term, which can be regarded as playing the role of either Landau and/or collisionai damping. Viscosity terms, whetiner real or artificial, were not included jnitially. These two equations are only the first in an infinite sequence of moment equations. The sequence is truncated here by assuming a scalar pressurc, either isothermal $P_{\mathbf{j}}=n_{j} \epsilon_{j}$ or adiabatic, $P_{j} \propto n_{j}$. Two equations such as $(2-1)$ and (2-2) are included for each piasma species. The fields which appear in (2-1) and (2-2) are the macroscopic ones given by Maxwell's equations. In the transverse gauge, these can be derived from the potential equations 


$$
\begin{aligned}
\nabla^{2} \phi & =-4 \pi e\left(n_{i}-n_{e}\right) \\
\frac{\partial^{2} \bar{A}}{\partial t^{2}}-2 \nu_{e m} \frac{\partial \bar{A}}{\partial t}-c^{2} \nabla^{2} \bar{A} & =-4 \pi c \bar{J}_{t r}
\end{aligned}
$$

where $\bar{J}_{t r}$ is the component of the current density transverse to the direction of propagation, and $v_{e m}$ the electromagnetic wave damping 1ate. The macroscopic quantisies which are the sources of $(2-3)$ and (2-4) are just tine ones calculated in (2-1) and (2-2), so t'a set $(2-1)-(2-4)$ comprise a closed description. These are the equations which will be hereafter referred to as the "reduced description formalism," or RDF for short. The derivation and differencing of the eq̨uations is discussed in Appendix A.

The above model was tested against the simulation SBS coefficients of Kruer, Estabrook, and $\operatorname{Sinz}{ }^{3}$. This was felt to provide a stringent test of the technique, for the correct steady state reflection coefficient required proper treatment of a variety of diverse phenomena, such as electromagnetic wave propagation and damping in a plasma, stimulated scattering from self-consistent plasma waves, saturation of the instability, and the hydrodynamic response of the plasna. Figure (2-1) shows the simulation values as solid circles and the reduced description values fit with the solid line, for an ion damping tus of about one-quarter the acoustic frequency. The reflection coefficients are remarkably consistent up to values on the order of fifty percent. Since the ion trapping observed in the 
simulations for the strong scattering cases enhanced the ion camping rate, the discrepancies do not seem at ald unreasonable. The field strength above wiich the reduced descriftion model predicts too much sc:3ttering serves to delineate the regime of validity of the technique, for a given linear damping rate. Inclusion of reflection dependent damping coefficients is an intriguing but untested idea. Lacking such physics, the code is probably accurate so long as trapping has not begun.

The collisional absorption appears as a damping term $(2-4)$. For a fixed, linear density gradient the total absorption coefficient in the geometrical optics limit can be evaluated in closed form, as was done by Dawson, Kaw, and Green ${ }^{4}$. Their expression is

$$
A_{A b s o r b}=\left(1-\exp \left(-\frac{32}{15} \frac{L}{c \tau}\right)\right)
$$

where $\mathrm{I}$ is the density scale length in centimeters, c the vacuum speed of light and $\mathrm{T}=7.59 \times 10^{8} \mathrm{e} / \mathrm{e} \mathrm{cr}, \epsilon_{\mathrm{e}}$ the electron temperat:re in $\mathrm{KeV}$, and $\mathrm{n}_{\mathrm{cr}}$ the critical density in $\mathrm{cm}^{-3}$. The absorption coefficients calculated with the reduced model equations agreed with (2-5) within a few percent over absorption between one to ninety percent (c.f. Table 5-1). This is excellent agreement, especially since the analytic therry neglected the absorption of the evanescent wave. Simulations have iiscovered several regions in which collective processes can efficiently absorb the lascr energy. Strong plasma turbulence can be excited near the critical density by either the 
Electrostatic instabilities or resonance abscrption, and near onequarter critical density by either Raman scattering or the two plasmon ( $\left(u_{\mathrm{p}}\right)$ instability. This absorption is driven by waves tranverse to the propagation direction by trapping in large amplitude Langmuir waves, or by nonnormal incidence, none of which can be expected to be represented by the model equations in a slab geometry. Such absorption may be able to be included by adding locally large coefficients to the damping terms, but it is not purported that the calculations give then self-consistently. 
SECTION II

Factors Affecting the Swelliag of Electromagnetic Waves Waves Near the Critical Density

The propagation of elertromagnetic waves in inhomegeneous plasmas has been previously studied, especially in connection with ionospheric radio wave propagation. A large numner of analytic solutions have been found for steady, unpercurbed conditions ( $: f$. Ginaburg ${ }^{5}$ ). In laser fusion plasmas the radiation intensity is of ten great enough to drive perturbations unstable, so the unpertarbed state has not elicited interest comparable to the "turbulent" plaswa state. The latter requires knowledge of the local field energy density, however, and near threshold or near the transition point for different nonlinearities, this quantity must be known with considerable precision. The complication then is that the local energy density near the critical density can be an order of magnicude greater than the vacuum traveling wave value even in steady, unperturbed plasmas. When effects such as collistonal absorption, nonlinear density profiles, and laser incoherency are considered, the situation becomes more realistle but the uncertainties about the local field density increase proportionately.

The reduced description technique proved an Inexpensive, simple tool for quantitativa study of unperturbed intensity patterns under a variety of condictons. The Alry equation is derivable from (2-4) by neglecting damping, assuming the potential varieg sinusoldally in 
time with frequency $\omega_{0}$, and the density linearly with position,

$$
\frac{\partial^{2}}{\partial p^{2}} \pm \rho A=0
$$

where $n(x)=(1-x / L)_{c r}$, and $p=\left(a_{o} L / c\right)^{2 / 3} x / L$. The solutions for (2-6) are Airy functions, and the intensity pattern for these functions is tabulated in many mathentatical raferences. The RDF calculations in a fixed linear density profile confim this Airy pattern. Figures (2-2) and (2-3) show a typical gradjent and intensity pattarn. Lnder realistic conditions the density gradient cannot always be expected to be linear, thoigh one hopes that it can be approximated as such in certain regions. The above analytic solutions do not carry over to gradients of the form $n_{n=n_{c r}}\left(1-x / L_{1}\right)^{p}, p \neq 1$. (This is an unproved assertion, but the fact that the power reries solutions are in terms of three point recurrence relations implies that closed form solutions are impossible.) An approximate analytic expression is suggested, however. Since $(1-\mathrm{x} / \mathrm{L})^{\mathrm{P}}$ approaches $1-\mathrm{px} / \mathrm{L}$ near the critical density (i e., as $x$ goes to zero) it is plausible that the pattern can be described by an Airy function with an effective scale length, L/P. This renomalization is reasonable only near the critical density, but this is the region of most of the swelling.

The steady intensity patterns were calculated in fixed profiles with $1 \leq \mathrm{p} \leq 6$ using the $\mathrm{RDF}$ equations. The parameter most perinent here was felt to be the peak intensity of the standing wave pattern normalized to the vacuum standing wave peaks. The calculated values 
of this ratio aro shown in Figure (2-4) plottes as circles, with tine solid I ine representing the peak values predicted on the basis of the effective scale length liry patcern. The simple theory can be seen to be consistently within ten percent of the observed valurs. This agreement is feit to be quite satisfactory. (It should be noted that the ratic show in the figure is net nectessid. the rust natural one from an experimental viewpoint, where one would normaly: measure the wis traveling intensity of the vacuum wave. This latter is a factor of', smaller than the peak standing wave va?ue, so comparison with the traveling value gives a swelling ratio on the order ten to twenty instead of three to four.)

Dissipation was meglected in deriving the Airy equation, but for laser fusion to be effective, absorption must be nonnegligible. This is net a serious coersight, however, for Ginsburg shows that dissipation with a linear position dependence can be included in such a manner that (2-6) again results, although $\rho$ is then a complex variable. For large absorption coefficients, the complex nature of $(2-6)$ should differ qualitatively from the reai analogous equation. Weak absorption, however, can be expected to give Airy-like patterns, with reduced amplitudes.

The collisional damping rate is quadratic in density, sc that it is not lin: ar in the spatial coordinate even for a linear density gradient. It is no longer possible to transform (2-4) into an Airy equation via a simple change of variables. The conclusions of the previous paragraph, therefore, need no longer be true. Physically, 
one might conjecture that the quadratic dependence of the danping would lead to most dissipation in the region most critical to the swelling, i e., near the critical density, so that again it is not obvious what the steady pattern would be.

The effect of damping was studied through the use of a damping term derived from Dawson, et.al 4 .

$$
2 v_{\mathrm{e}}=\frac{7.03 \times 10^{-4}\left(\frac{\mathrm{n}}{n_{\mathrm{cr}}}\right)^{2} z}{\theta_{e}^{3 / 2}}
$$

where $\mathrm{n}_{\mathrm{cr}}=10^{21} \mathrm{~cm}^{-3}$ has been assumed, $z$ is the average conizution state, taken as unity hère. For a given scale length $\mathrm{L}$ and gradient power-law, the only avallable "free" parameter is the electron temperature. The electron temperature is not actually iree, since it is detininec by the balarice between energy gained from the lasex and energy lost hrough condaction, radiatior, and expansion. Nevertheiess, if it is kept in mind that the actual temperature must be calculated" self-consistently, it seems reasonable to use this parameter to vary the tetal zmount of absorption.

A series of calculations were perfonned in fixed linear profiles which had a scale length of 10 vacuum wavelengths. The swelling factor was again measured as the ratio of the first peak below criticaldensity to the yacuum peaks. This swelling factor was found to be very closely the same as the firy factor, although the field was reduced in overall magnitude. The percentage reduction in the "ritensity at the first peak is plotted versus the percentage absorbed in 
Higure (2-5). The calculated values have beer least squares fitted to give an empirical expression for the peak,

$$
I_{\text {eff }}=\left(.44-.56 e^{-K_{\mathrm{T}}}\right) \mathrm{I}_{\mathrm{o}}
$$

where $k_{T}$ is the argument of the exponential in $(2-5)$. The peak intensity depends roughly linearly on the reflection coefficient for absorption less than fifty percent. For larger absorption the peaks fall progressively below (2-8), because the reduced back signal is no longer large enough to form a complete standine wave. The pattern is clearly not a standing wave when the absorption has reached ninety percent. Even with near total absorption, however, thr peak is rediced by only twenty percent from its vacuun standing wave value. Swelling near the critical density partiaily compensated for the attenuation. These are also Ginzburg's conolusions for the highiy absoroing case, in which he proposes a mirror surface to estimate the structure of the field in the plasma. The expression derived there agrees moderately well with the calculated values when the absorption is high. The shape invarjance of the intensity pattern has also been confirmed by Coldman, Dubois, and McKinnis ${ }^{6}$, using a different numerical technigue.

Because actual density profiles are frimed by the interaction of the expanding plasma with the laser, the profile need not be Iinear over any significant distance. To model this effect, calculations were also perfonied in nonlinear power law profiles $(p=2,4)$. Those 
results are also shown in Figure $(2-5)(p=2, \Delta$, and $p=4,0)$, where the reduction factor is plotted as a fluction of total absorpion. It is found that the factor is within about ten percent of the curve in the figures for a wide variety of scale lengths and profile shapes.

A more intrinsic factor which should affect the stelling of electromagnetic waves is the lack of coherence of the incident laser beam. This partial coherence should "smear" the standing wave nattern tc some extent. Since the average energy from the laser is not alcered by finite bandwidth, the smearing should decrease the peaks and increase the nodes on the average. Figure (2-6) shows a calculated average bandwidth which exhibits just these features [c.f. Figure (2-2)]. The uncertainty about the pattern then is the quantitative dependence of the peaks on the bandwidth.

This problem can be modeled by averaging an ensemble of Airy functions. The distribution of Airy functions can be roughly related to the frequency spectrum as follows. Define the random variable $\tilde{w}$ such that $\omega=w_{0}+\tilde{\omega}$. For convenience suppose that the incident signal has a gaussian line shape, so the frequency distribution is

$$
\sigma(\omega)=\frac{1}{\sqrt{2 \pi} \Delta \omega} e^{-\omega^{2} / 2(\Delta \omega)^{2}}
$$

norialized to unity. Now the Airy function scales according to the normalized length 


$$
a(w)=\left(\frac{c^{2} L}{\omega^{2}}\right)^{\frac{1}{3}}
$$

If this farmeter is separated into mean and fluctuating imponents, one can easily identify

$$
\begin{aligned}
& a=a_{0} i a, \\
& a_{0}=\left(\frac{c^{2} L}{\omega_{0}^{2}}\right)^{\frac{1}{3}} \\
& \tilde{a}=\frac{2}{3}\left(\frac{c^{2} I}{\omega_{0}^{2}}\right)^{\frac{1}{3}} \frac{\tilde{\omega}}{\omega_{0}}
\end{aligned}
$$

provided $\sqrt{\left\langle a^{2}\right\rangle} \ll a_{0}$. The ensemble average of Airy functions, $\left\langle A i^{2}\left(X_{0}\right)\right\rangle$, with differing scale lengths, evaluated at $x_{0}$ is therefore

$$
\left\langle A i^{2}\left(x_{0}, a_{0}\right\rangle\right\rangle=\int_{-\infty}^{\infty} \operatorname{daff}(\tilde{a} ; \Delta a) A i^{2}\left(x_{0}, a_{0}+\widetilde{a}\right)
$$

where $f(a ; \Delta a)=\frac{1}{\sqrt{2 \pi} \Delta a} e^{-a^{2} / 2(\Delta a)^{2}}$ and $(\Delta a)^{2}=\frac{4}{9}\left(\frac{c^{2} L}{\omega_{o}^{2}}\right)^{\frac{2}{3}}\left(\frac{\Delta \omega}{\omega}\right)^{2}$.

The time averaged peak amplitude squared for $L=10 \lambda$ and $\mathrm{p}=1$ is shown in Figure $(2-7)$ as a function of relaive bandwidth $\Delta \omega / \omega_{0}$. The bars are due to the fact that temporal fluctuations in the stochastic model for the laser bandwidth lead to dispersion around the 


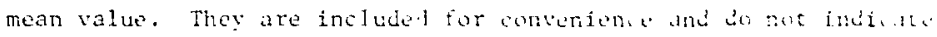
any uncertainty in measurement. The solid line in the finger is the

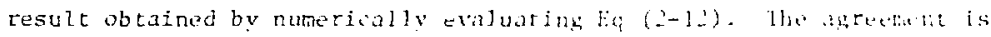

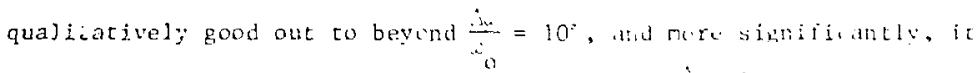
is in good quantitative agreement in the regime $\cdots .5 \%$. High purror lasers needed in laser fusion may not be able w" "poralte with wa large a bandwidth. Consequenty, ive perent bancidel is probuly close to the upper limit for prastical applicalioms.

The agreement between the model and calculation encingaged us to study the scaling of the swelling factor with sysers iength. The results are shown in Figurs $(2-8)$, for different binciwidts. The r.odel predicts significant reduction in the pe?k amjitude at rooust bandwidths for the longer systems. The redued deteription citluittions agreed moderately woll. As an example, it $I=42$, the model

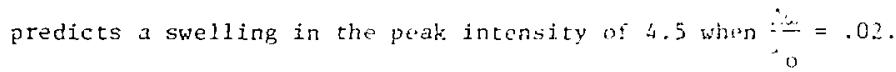
The measured swelling factor was $4.8 \pm .3$. Agreement to vitlin turi percent certainly constitutes satisfactory accuracy for a moded ats simple as this. The fact that it ives roughly the correct scaling with both bandwidth and dinsity scale length indicates that the results shown in Figurt (2-8) are probably a useful measure of the reduction in swelling with bandwidh in realistic systems.

It should be noted that the magnitude reduction hers amounted only to a factor of about fifty percent. The effect of bandwidts on parametric instability thresholds, however, tends to be order of 


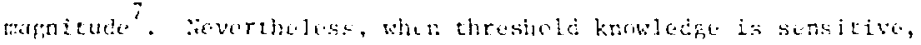
bandwidth reducton in the incersiay may noet co be consictered. 


\section{SECTION III}

Self-Steepening of Density Profiles Near the Critical Density

Kicker has proposed that the plasma profile will steepen at the Eitical densicy because of the zero-order radiation pressure. This problem is especially anenable to study chrough the reduced description, since "unwanted" physics can be omitted to isolate a specific effect. In fact, the reduced description methodology is so attractive for this case that lee, et a1. 9 have used similar techniques already to investigate steepening. Kidder derived a simple analycic expression for the density jump. This, along with Lee's results, indicates that there is ample data to which these calculations can be comparad. Kidder balanced radiation sressure against the plasma pressure at the classical turning point of the electromagnetic field. He assumed that the plasma flows through this point at the ion acoustic speed, $c_{s}$. When a laser impinges on this surface, however, it is reflected. Conservation of momentum suggests that the plasna must take up the excoss momentum, so that the net flow is inhibited.* The resulting density jump is then given by

$$
\frac{\Delta n}{n_{c r}}=\frac{a+\sqrt{\alpha(4+a)}}{2}, \quad \alpha=\left(\frac{v_{o s}}{v_{t h}}\right)^{2}
$$

\footnotetext{
Fonservation of flux indicates that the lowered density below the critical density should be supersonic. Such a velocity profile will lead to a larger tensity jump titan is given by (2-13).
} 
Dissipation near the critical density, of course, will reduce the amount of reflaction, but this effect can amount to no more inan a factor of 2 in the radiation momentum exchange.

The RDF calculations showed definite steepening at the critical density, but also revealed certain numerical problens. Figure (2-9) shows the upper and lover density "shelves" as a function of vacuur. field strength, $\left(V_{o s} / V_{t h}\right)$. The upper levels are entirely consistent with those reported by lee. For sufficiently low ion damping, indicated by $\Phi$, the lower density was also in adequate agreement vil: Lee's pubished data. At larger ion damping magnitudes, however, the lower density was consistenty too high. The reason for this is related to the differencing of the ion flux equation, (2-2), and the form used for damping.

The simple theory derived by kidder assumed inicially that the plasma flowed sonically. This is only true for free expansion, but in the calculations, it was often subsonic by an early point, and decelerating. This is clearly nonphysical but readily understandabla if one examines the leading terms of the differencing used in (2-2),

$\Gamma_{j}^{t} \cdot \Delta t=\Gamma \frac{t}{j}\left\{\frac{1-.5 \Delta t v}{1+.5 \Delta t v}\right\}-\frac{\Delta t}{(1+.5 \Delta t v)}\left\{\left.\frac{\Gamma^{2}}{n}\right|_{j+\frac{1}{2}}-\left.\frac{\Gamma^{2}}{\Gamma}\right|_{j-\frac{1}{2}}\right\}^{t+\frac{\Delta t}{2}}$

where only the convective term is shown. At low enough density, the second term on the RHS of (2-14) can be arbitrarily small. This is primarily the term that drives the low density expansion. Then since 
the time step is fixed and finite, it is possinlo for the fixed decrease in the ilux, i a, the first term on tine Ris, tu wexed the comvective increase. The not result is that the tux at that pisition suffers a net decrease. It is deceloratod. Inhibition of tho bow censity ilow cuentually propagates throughoul the entire espansion, slowing the expansion to nonphysically low sneteds. For subsonde speeds. Kidder's derivation would predict a smaller density jum, Hence, the lower density sheli is too high when $2=.0025$ os while at $v=.0002 \omega_{0}$, the expansion is always at least sonic, and the predicted density jump is observed.

Figure (2-10) shows a typical steepened density ior $\left(v_{o s} / v_{t h}\right)=0.5$ and $v=.0002 \omega_{0}$. The resolution of the density itup is degraded by the modest at ount of expansion which has occurred ar the time of this "snapshot" Infortunately, the greatly reduced ion damping allowed stimulated Brillouin scattering at even this 1ow purp level. The time averaged field pattern shows this unambiguously at later times, though not at the time shown in the figure. Whenever. Brillouin scattering did not interfere with steepening, however, the density jump at the critical density was given approximately by (2-13). Figure (2-11) shows Eq (2-1.3) compared with the medsured density jump. The agreement is good, despite the fact that (2-13) should be no better than a lower bound on the density jump.

The reduced description techrique overall performed adequately in studying profile steepening at the critical density. Certain model dependent characteristics were found which could seriously alter the 
results. Although these werc jdentifjed and avol bed, they illestrabed that this tecindege, flenible though it is, cruld ate be applied natvely to any problem. As with any numerica retetod, roliable results wert obtainable only af ter the technique ilself was understoria encurgh that improper solutions could be recopnized and discarceci. 


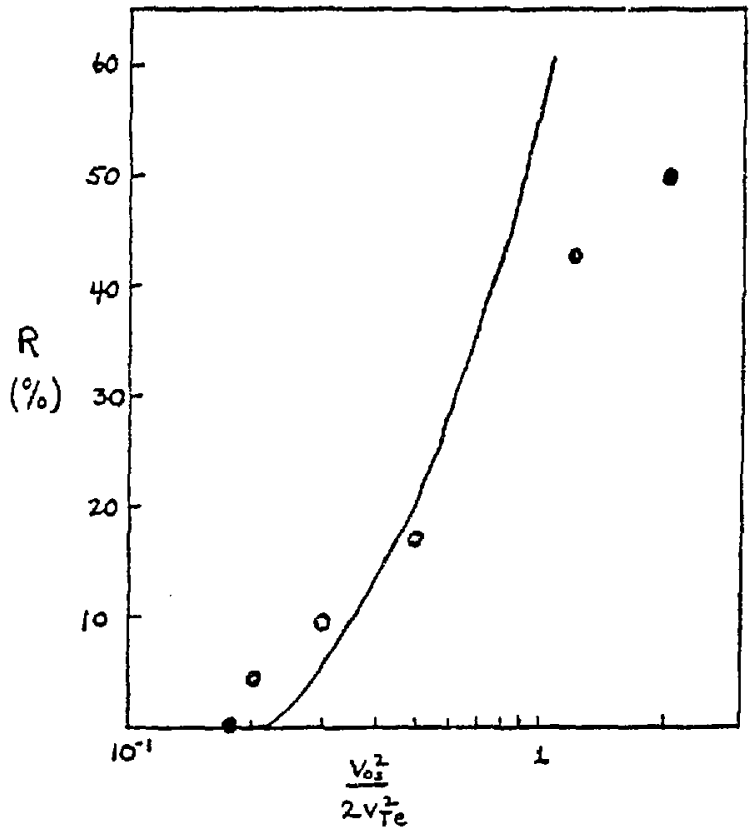

Figure 2-1 Stimulated reflection coefficients as a function of normalized field strength. Points are taken from Ref 3, solid line shows RDF coefficients for similar parameters. 


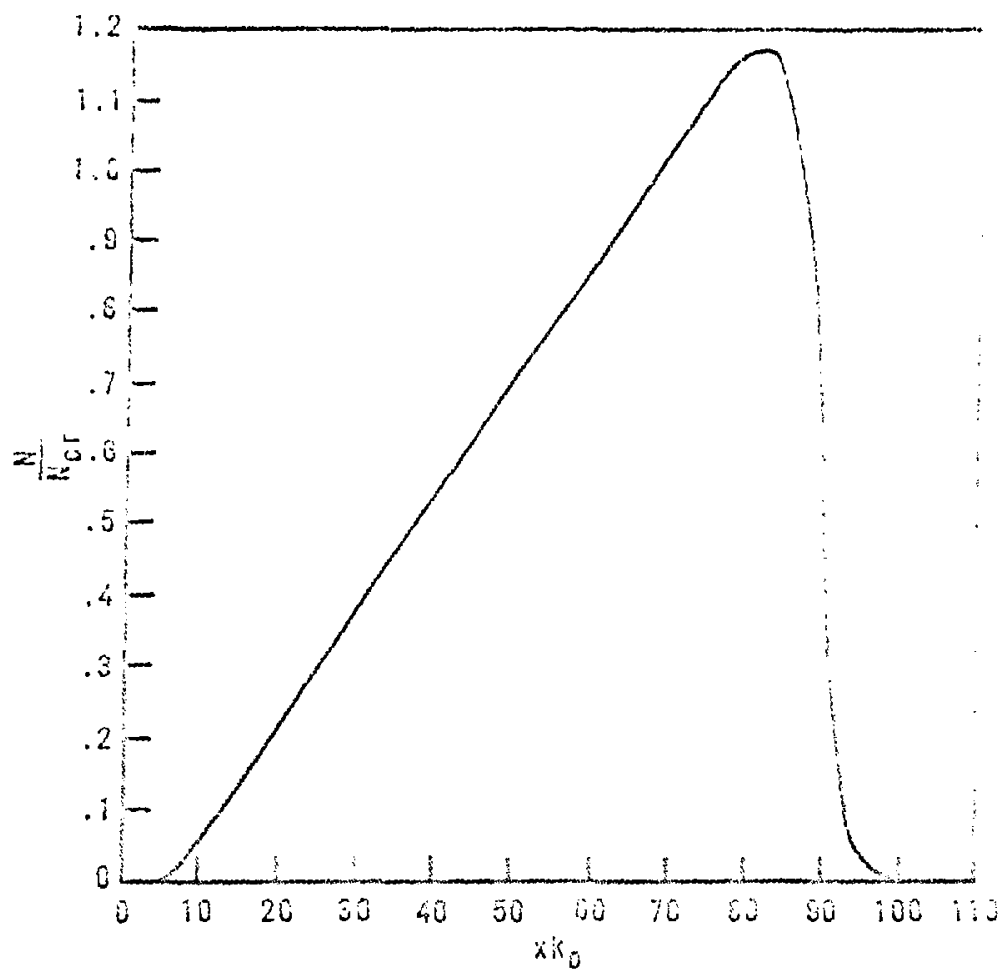

FIGURE 2-2 Typical density profile used in swelling calculations, $m_{\mathrm{e}} / M_{i}=0.0$. 


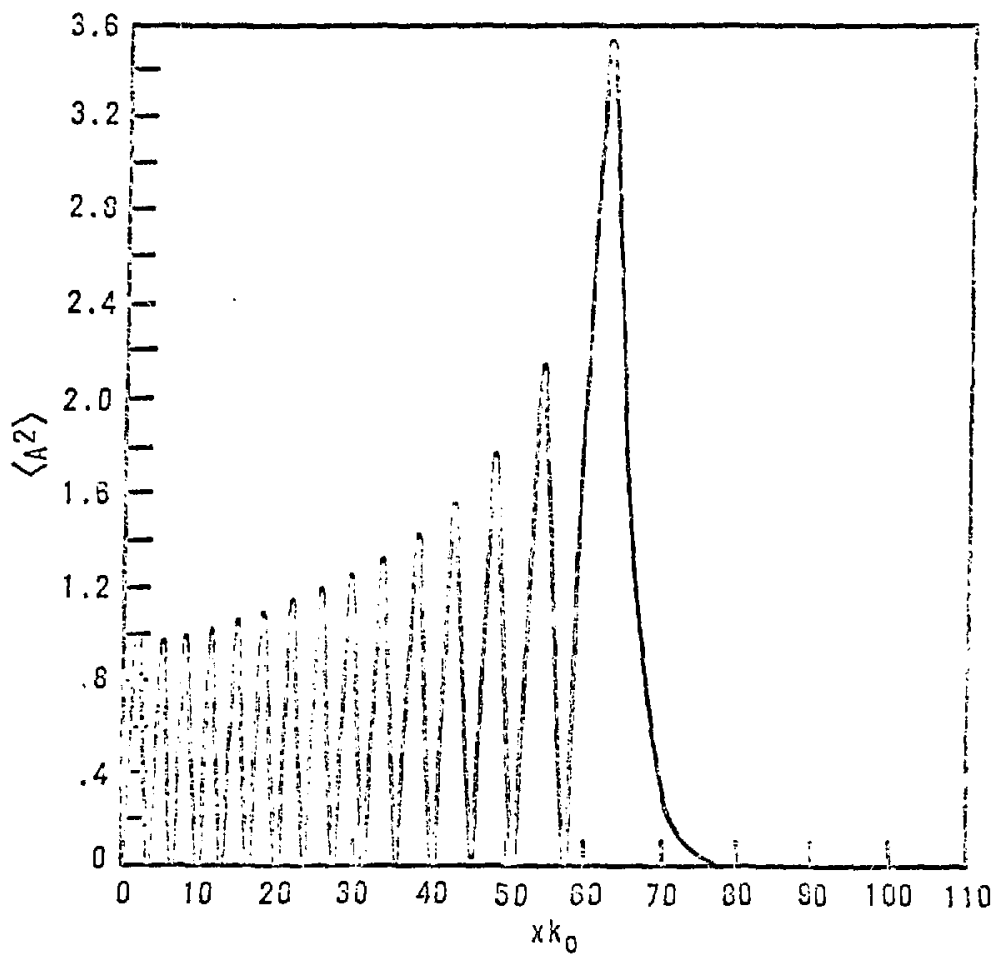

FIGURE 2-3 Time-averaged field pattern as a function of position. $\left(v_{o s} / v_{t h}\right)^{2}=0.5$. 


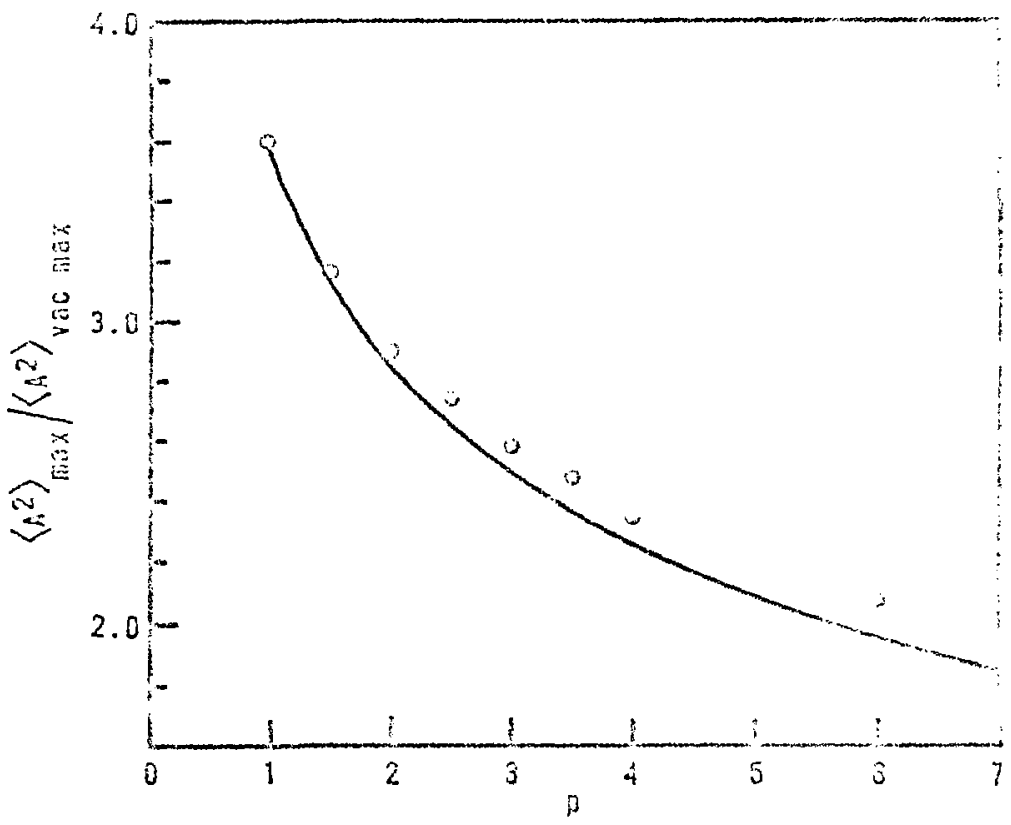

FIGURE 2-4 Sweliing factor (o) as a funciion of powerlav density profile, $n \propto(x / L) p, L=10 \lambda$. Solid line indicates Airy-sweliing for effective Scalelength L/p. 


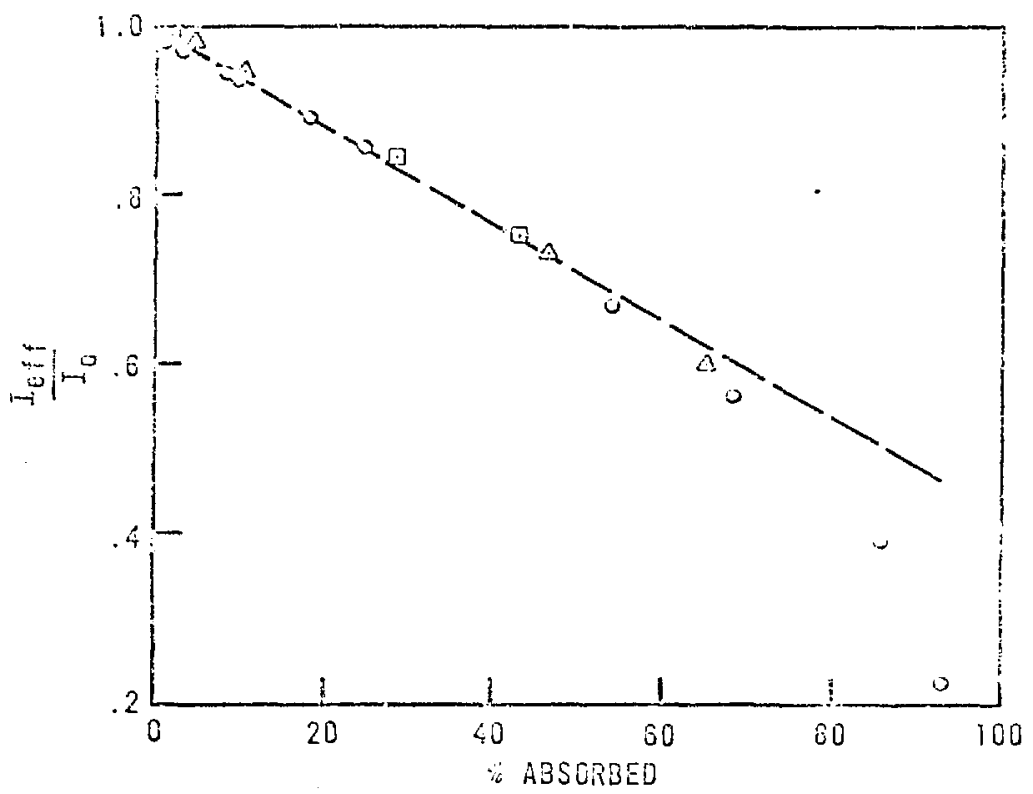

FlGLRE 2-5 Normalized standing wave incensity as a tunctin of collisional absorption (inverse buenstrahl(in:?), $p=i(\bullet), p=2(i), p=i(\because)$. 


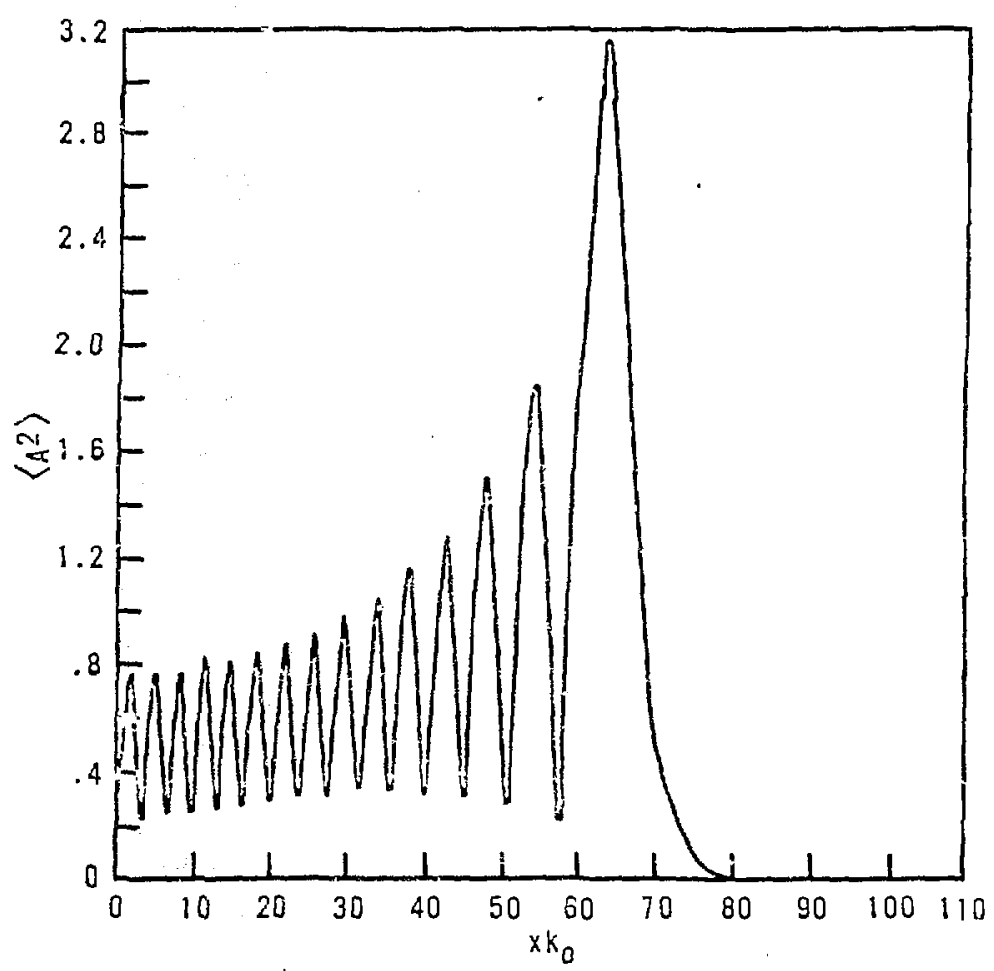

FIGURE 2-6 Typical intensity pattern for a slighily incoherent pump (time-averaged), as a function of position. $i w / u=0.02$. 


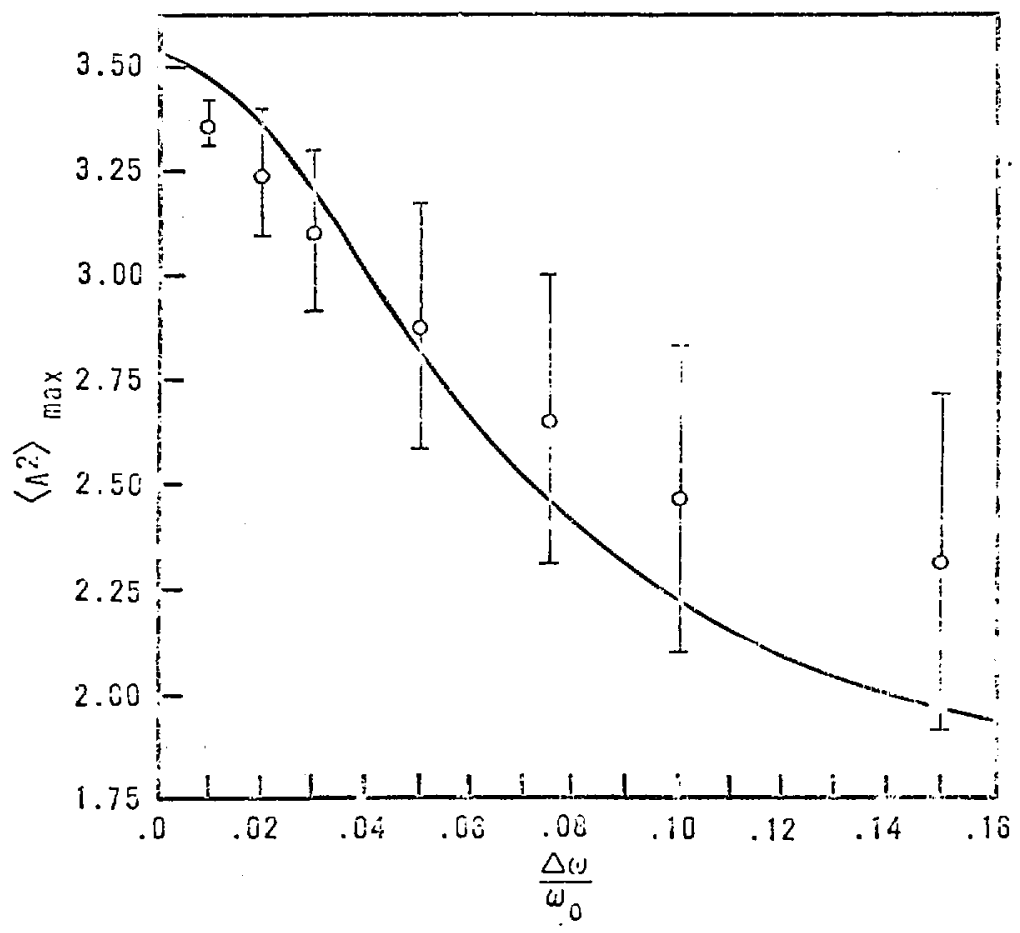

FIGURE 2-j Swel1ing factor as a function of laser bandwidth, $\quad\left(\mathrm{V}_{\mathrm{Os}} / \mathrm{V}_{\mathrm{th}}\right)^{2}=0.5, \quad \mathrm{~L}=10 \lambda_{\mathrm{o}}$. 


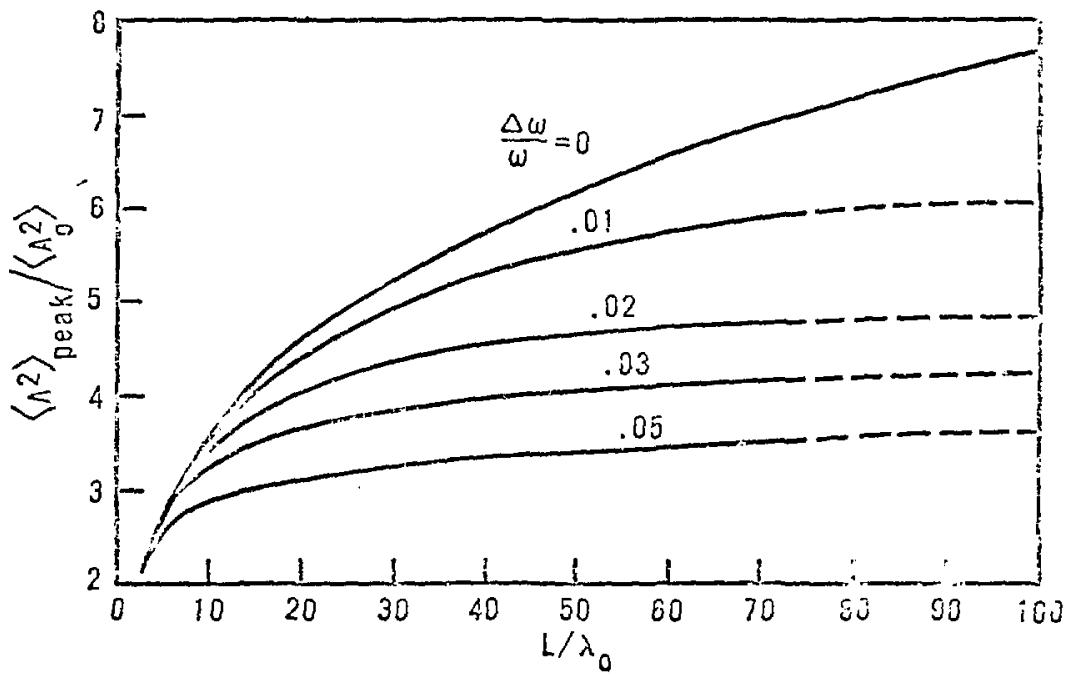

FIGliRE 2-3 Swelling factor as a function of linear density profile length, for $\Delta \omega / \omega_{0}=0.00,0.01,0.02,0.03,0.05$. 


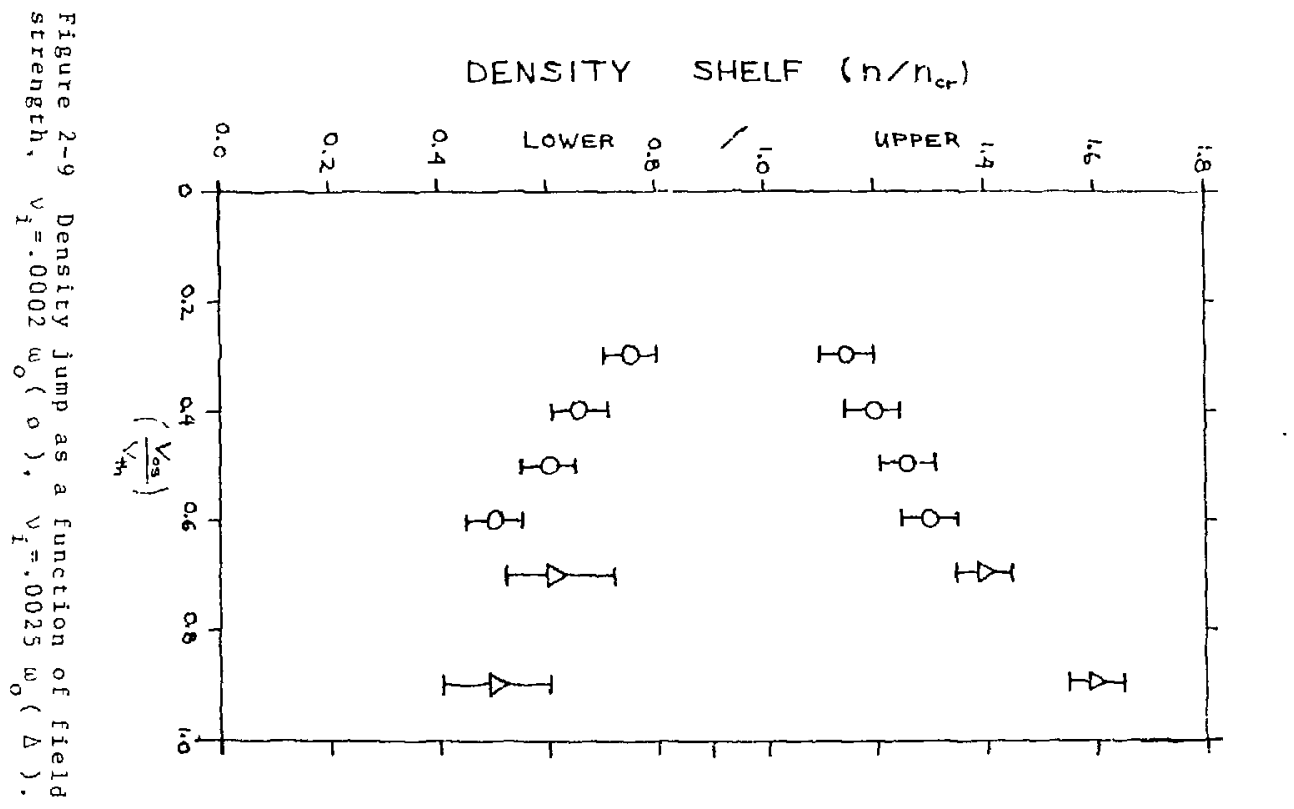

w 


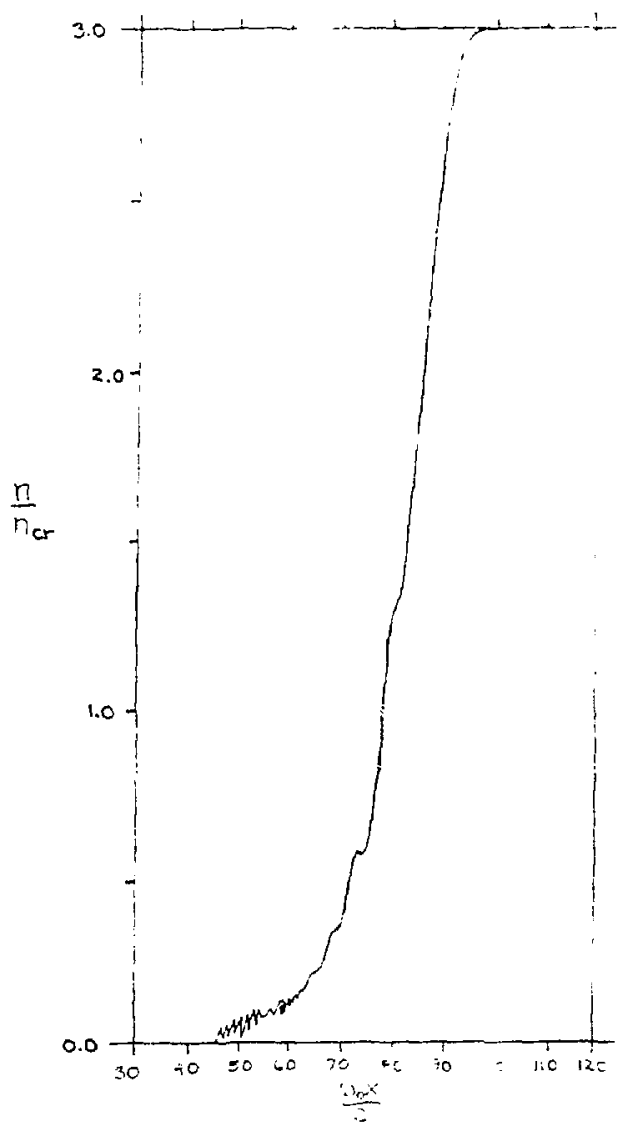

Figure 2-10 Density profile at w $t=200 \%$. $\left(V_{o s} / V_{i h}\right)=0.7, \quad v_{i}=.0002 \quad a_{0}^{2}, m_{e} / R l_{i}=0.0:$ 


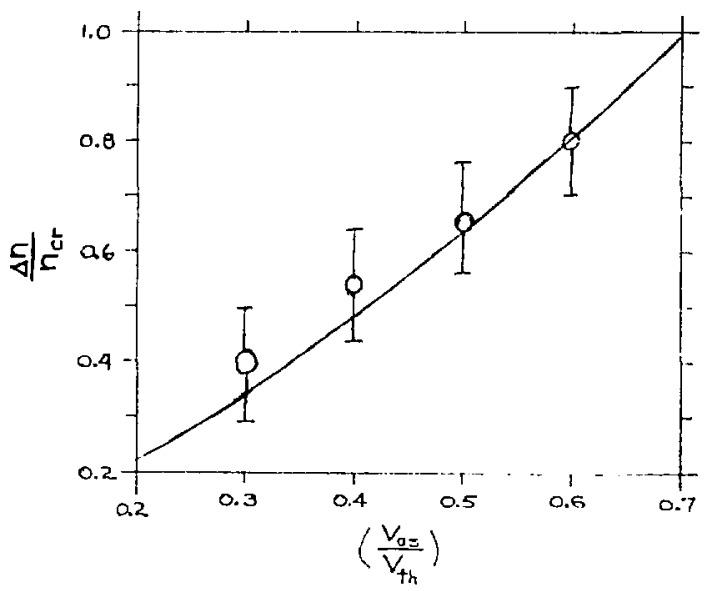

Figure 2-1l Calculated density jump as a function of field strength, $m / M_{j}=0.01$. Solfd line is Eq(2-13). The error hars reflect the considerable uncertainty In determining upper and lower shelves from computer generated graphics. 


\section{CHAPTER 3}

Stimulated Brillouin Scattering and Mode Coupling:

Nonlinear Evolution of a Parametric Instability Process

Stimulated Brillowin node coupling effects will be studied in this chapter. This does not refer to the linear instability phase of the process 10,11 , which is certainly a node coupling effect, but Inctead to the interaction of the large amplitude waves cre ited by the scattering. Since the exponential growth is not of primary interest, attention will be focused on the post-saturation, nonlinear evolution of the waves. The recuced plasma formalism is felt to possess several distinct advantages over simulations for such a study. Among these are much shorter calculation times, much lower noise levels, and the ability to isolate particular physical processes.

The rationale for such an investigation are (1) there is ample evidence from laser plasma scattering experiments that some mechantsm(s) broaden the electromagnetic wave spectrum $12,13,14,15$, (2) to the best of our knowledge, SBS mode coupling has not been previously Investigated, and (3) related to the above, understanding the source of the broadening may provide instghts into the wicrodynamics inside the plasma, which are otherwise inaccessible. Clearly, simulations and analytic theory have shed conslderable Iight ${ }^{*}$ on the Brillouin scattering process, usually whth regard to the level of reflection. Nevertheless, mode coupling studies of other parametric instablifties

\footnotetext{
Piease note pun.
} 
(i e. the parametric decay instability) have shown that they are rich in wave-wave interactions. There is every intuitive reason to believe that conditions can be found in which they are also propitious in Brillouin scattering. Stfmulated Raman and Brillouin studies in solid state media moreover have long been acconpanied by complex spectra (c.f. Bloembergen ${ }^{16}$ ). The fact that the claracteristic spectra, asymetrically oroadened toward longer wave lengths, are reported in virtually all laser plasma experiments (in which the spectrum was measured) suggests similar mechanisms may be occurring.

The chapter is ordered as follows: In Section I, a wave coupling version of the recuced description formalism (RDF' is described and used to identify the qualitative characteristics of Brillowin wave coupling. Some of the quantitative wave field features are examined near threshold and the extrapolation to higher intensities is inferred. This section involves considerable detail since it is the basis for evaluating more realistic, inhomogeneous calculations later. Still, the topic is somewhat esoteric, and the applicationsminded reader may wish to pass over this section lightly. In Section II, the infinite medium assumption is relaxed to study the effect of finite length on the mode-coupling. Inhomogeneous density profiles aro also examined. Finally, in Section III, the possible applications of these calculations are illustrated by concentrating on a series of scattering experiments conducted by an NRL group, Ripin, et al. 15 . 


\section{SECTION I}

Homogeneous Brillouin Scattering Studies

Before proceeding, a historical tootnote seems in order. Solid medium laser experiments were the first to see the pronounced asymmetrical broadening of scattered spectra ${ }^{16,17,18}$. Investigations of SBS in solids, liquids, and gases have all observed such spectra for sufficiently high intensities. The high intensity is apparintly crucial*, for the spectrum changes from a series of discrete Stokes lines at moderate intensities into a diffuse, broadened spectrum at the greater intensities. Although such phenomenon have been studied for nearly a decade within the framework of nonlinear optics (i e., cubic field nonlinearities), it is felt that the properties of a dense plasma are sufficiently unique that the earlier results are not directly applicable. They are still useful insofar as they indicate that sfectral broadening can be the nonlinear result of a known linear process, that is stimulated process.

The first ROF calculations were corducted in homogeneous, periodic systems, Although end effects were omitted, the improvei spertral resolution was an enormous advantage in following the modecoupling. It was convenient in this situation to Fourier analyze the RDF model equations, and so observe the evolution of the spectrum

\footnotetext{
${ }^{*}$ Early experimental observations of the spectral broadening occurred during inadvertent selfffocusing and filamentation.
} 
direct]y. The equations $(2-1)-(2-4)$ were thus reduced to tho sets of coupled wave equations, though each mode was intrinsically coupled to all other modes. The algebra in deriving the sets is straight fortard but tedious and so has been delerred to ar appendix. The final equations for the electromagnetic and ion density modes are

$$
\begin{aligned}
& \ddot{A}_{i}+2 v_{e k} \dot{A}_{k}+\omega_{k}^{2} A_{k}=-\omega_{0}^{2} \sum_{\hat{k}^{\prime}} n_{k} A_{k}-k^{\prime} \\
& \ddot{n}_{k}+2 v_{i k} \dot{n}_{k}+\omega_{i k k}^{2}=-\frac{1}{2} n_{0}\left(\frac{v_{0}}{v_{t h}}\right)^{2} \omega_{i k}^{2} \sum_{k} A_{k} A_{k}-k^{\prime}
\end{aligned}
$$

where $w_{k}^{2}=\omega_{p e}^{2}+k^{2} c^{2}, w_{i k}^{2}=\frac{6}{M_{i}} k^{2}$. It should be noted that this technique retains phase, as well as amplitude infoimation, and so should be quite general ${ }^{19}$.

To isolate the dominant wave-coupling mechanisms, a series of calculations were done in which the intermode k-spacing, the number of modes, the ambient density, and the pump fieli strength, $\frac{V_{\text {os }}}{v_{\text {th }}}$ were varied. Changing system length due to plasma exponsion or too large an intermode spacing (i e., too short a system length) may mask such interactions in finite simulations. It is possible that particle simulations are suitable for this type of study, but the point is moot until such an attempt is made. Using the set (3-1a) and (3-1b), two distinct types of mode-mode interactions were observed to be particularly strong in the saturated state of the SBS instability. 
The first mechanisn is simply hamonic generation, driver by the large amplitude waves. For sufficiently strong pump, and density close to critical, the coupled wave calculations show strong spatial harmonic generation at $3 \mathrm{~K}_{0}, 5 \mathrm{~K}_{0}, \ldots$ for the high frequency waves, and $4 K_{0}, 6 K_{0}, 8 K_{0}, \ldots$ for the ion waves. This prucess can be explained as a nonresonant, for.ed oscillation. Vieved strictly in k-space the SLS instability transfers energy from a large amplitude lectromagnetic wave at $k_{0}$ to another electromagentic one at $-k_{0}$ and an ion accustic wave at $2 \mathrm{~K}_{\mathrm{O}}$ ( $\mathrm{i}$ e., forward traveling with respect to the pump), with the latter also carrying off the bulk of the momentum. This proress is resonant, in that it not onjy satisfies $\overline{\mathrm{K}}_{0}=\dddot{\mathrm{k}}_{\mathrm{en}}+\overrightarrow{\mathrm{k}}_{i a}$, but also $\omega_{0}=\omega_{\text {em }}+\omega_{1 . a}$. Once the decay products have reached large amplitude, however, one should consider the possibility of nonresonant coupling to new waves. For the present case, quasiresonant is a more descripLive term, since we will. still seek triplets such that $\sum_{i=1}^{3} \vec{k}_{i}=0$. though frequency mismatches may be allowed.

Let us exarine the $3 K_{0} \in$ tectronagretic wave as a typical example. Since it is the density fluctuations which couple the electromagnetic waves, and since a large amplitude lon wave does exist at $2 K_{0}$ (by assumption), the most obvious triplet is the coupling of the forrard traveling pump and the forward traveling ion acoustic wave to a $3 K_{0} \mathrm{EM}^{\mathrm{k}}$ wave. Except in a physically insignificant region near the critical density, we find $w_{3 \mathrm{~K}_{0}} \neq \mathrm{u}_{\mathrm{a}}+\omega_{\mathrm{ia}}$. The process involves the pumping oi a wave whose natual frequency, is $\omega_{3 k_{0}}=\left(\omega_{p}^{2}+\left(3 k_{c}\right)^{2} c^{2}\right)^{1 / 2}$, with a pump whose effective frequency is $\omega_{0}+\omega_{i a}$, and effective amplitude is 
$\omega_{n}^{2} n_{i k} A_{0}$. A forced trarmonic oscillation such as this will eventually drive the $3 \mathrm{k}$, wave to a steady amplitude, which is easily calculable. Before we insert observed values to actually rest this model, we should consider one other possible triplet that could lead to a $3 \mathrm{~K}_{0}$ wave.

It is well known that a large amplitude ion acoustic wave can effectively dec..y into its higher harmonics, as long as $k \lambda_{\mathrm{b}}$ < 1 . As $k_{\mathrm{D}} \approx\left(\frac{\mathrm{V}_{\mathrm{ch}}}{\mathrm{c}}\right)\left(\frac{\mathrm{kc}}{\omega_{0}}\right)\left(\frac{\mathrm{n}_{\mathrm{cr}}}{\mathrm{n}_{\mathrm{o}}}\right)^{1 / 2}$, the inequality is very well satisfied near the critical de: sity. The ion acoustic wave will, therefore, expend its energy at a given rate, depending on its amplitude, in generating higher harmonic ion waves, independent of any coupling with the high frequency wave. Once an appreciable amplitude is attained by the second harmonic wave, that is $4 \mathrm{~K}_{0}$, it can in principle couple a $-\mathrm{K}_{0}$ electromagnetic wave to a $3 k_{0}$ electromagnetic wave.

A typical spectrum showing harmonic generation is shown in Figure 3-1. The initia? density in this calculation was $.935 \mathrm{n}$, the initial pump $s$ ength was $\left(V_{o s} / V_{t h}\right)^{2}=.25$, with pump depletion allowed, and to minimize ion Lardau damping $\theta_{e} / e_{i}=100 . A$ small signal in this ase was even observable at $5 K_{0}$, though reduced in intensity by roughly 5 orders of magnitude. The relative importance of the two coup ing mechanisms can be judged by comparisons with the forced oscillation model. A straightforward derivation yields the steady RMS level of such an oscillation.

$$
A_{k}^{2}=\frac{\omega_{p e^{n} i}^{n}\left(K+k_{o}\right) A_{k}^{2}}{\left(\omega_{k}^{2}-\omega^{2}\right)^{2}+4 \nu_{e}^{2} \omega^{2}}
$$


TABI.E $3-1$

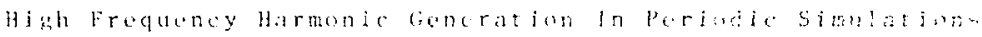

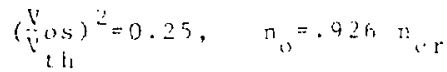

$$
\begin{aligned}
& { }_{2 k}^{2}=3.5 \cdot 5 \times 10^{-3}
\end{aligned}
$$

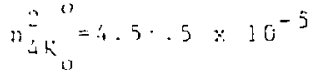

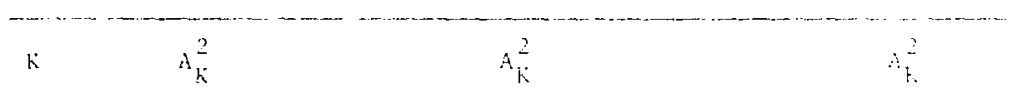

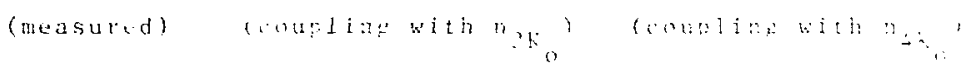

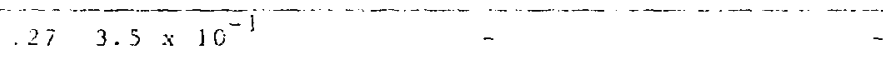

$.813 . \times 10^{-3} \quad 3.85 \times 10^{-3}$

$1.351 .2 \times 10^{-5} \quad 3.12 \times 10^{-6} \quad+.6 \times 10^{-6}$ 
where $\omega_{k}=\left(w_{p}^{2}+(k c)^{2}\right)^{1 ; 2}, \omega=\omega_{k}+w_{i k} \pm k_{0}$, and $v_{e}$ is the damping incremert on $A_{k}$. Lsing the observed values for $n_{i k}^{2}$, Table $3-1$ shows the agreement between the observed and predicted levels for these harmirnics, assuring that th coupling is between the $\underline{K}_{0}$ pump wave and $2 \mathrm{~K}$ ion wive. The lariest di crepancy in this limited sample occured for a case in wich the magni ude of the second harmonic ion wave vas within a factor of four o: the primary. This suggests that the second coup:irg scheme can b: quite effective if the density wave harmonics are powerfully driv $n$, though the lower order scheme would be the nore cotionly encountexs one.

The second mech: sm encointered in the calculations might be called repered scattering ${ }^{1 .}$. Once the pump has driven a backscattered wave tis a litge amblitude, the secondary wave can itself act : an SBS putap. The new electromagnetic wave (i e., secondary decay product) is propagating along with the original pump wave, but downsififed by approximately sice the Erequency of the acoustic wave*. This secondary beckszi: tering will, of course, also genterate backlard traveling ion waves, .. d once ion waves of comparable ragnitude hive been created in : oth directions, the possibility of quasirasonat couplings shouid b. considered. If one could naglect

\footnotetext{
"It should be noted that the fres ency shife is not necessarily that of a normal inn acoustic wave. 'l' deviation from the normal mode frequency may bi fither positive. regative depending on the exact wavenumber. The measured irequen shift is consequenty an unreliahlo indiator of the density or watenumite af the original scatering everte.
} 
all but the last hackscattering event, towter, it is flusible trat this wave, too, could drive SLS if it exceeded the aprrofrite thetsit old. There is no a prtovi reason why the other weres car be neglucted, but as will be cited helck, calculations indicate that the systor evolution is conststert with this hypothests. The procedute thon is that a series of consecutive backscatters, i $e$, in alternating directions, leads to a sequence of infes separates by twice the acoustic woxe frequency. The process can be entinuted eithe- by competing aude-mode interactions or when the dissipation rale inteEratec over theentire spectrum equals the rate at which energy is fec into the spectrum from an external source.

Stimulated rescattoring is cleariy evident in Figure $3-2$, the late time spectrum of a launched wave calculation for which ${ }_{0}=.5 \mathrm{n} \mathrm{cr}^{\circ}$ $\left(v_{c s} / V_{t h}\right)^{2}=.25, e_{c}=2 \mathrm{keV}$, and the syster. lergth, I. $=40 \lambda_{0}$. The spectrun is otserved to broaden in time in exactiy the namicr describec above. Although additional waves have also reached significant levels by this time, the contrast ieve] betwen the rescittered woves and the purp has been suffictently jepleted so that. ne further broacining uncurs, but the spectral widh has already attaired roughly ejght perce th of the original frequency $\left(A_{0} / \omega_{c} \sim 1 \%\right.$ for $\left.\mathrm{m}_{\mathrm{e}} / \mathrm{M}_{\mathrm{i}}=1 / 1826\right)$. Furthermore, somewlat later in the calcularion, off-rescnant cotiping, facilitated by the broad spoctrum, filled in the spectral "valleys." Thus, the occurrence of repeated scattering would not necessarily leac to measurement of a discrete Iine spectrum. 
Figure 3-3 shows both hamonic generation and repeated scattering in a calculation in which $\mathrm{n}_{\mathrm{o}}=.935,\left(\mathrm{v}_{\mathrm{os}} / \mathrm{V}_{\mathrm{th}}\right)^{2}=.5 \mathrm{n}$, aיd $\mathrm{L}=40 \lambda_{\mathrm{o}}$. Uarmonic generation occurred initially, but the slow broadening due to repoated scattering continued after the former had ceased. During the repeated scattering, the original pump wave was gradually depleted, as expected. The three wave coupling manifested itself in the fact that the newly driven waves only induced transient depletions in the waves directly involved in pumping. Since the pump waves were strongly coupled to their respeciive sources, they were quickly restored to their previous leve1. Consequently, the evolving spectrum was immune to large fluctuations. As Figure 3-4, a later spectrum from the same calculation, shows though, large and dramatic alterations could be induced. The spectral changes are correlated with the appearance of a band of up-shifted, higher-k waves, and these waves are clearly dravitis energy only from a narrow region of the spectrum, centered around the wavenumber of the original pump wave. The spectral broadening, moreover, assumes an entirely different character after this, diffusing at a rapid rate to the lowest wavenumbers in the calculation. Epshifted waves, up to three times the original wavenumber, are also evident. The role of the blue shifted waves in the broadening was not obvious within the contcrtof simple mode-coupling discussions, but can be clarified if they are identified with a purely growing branch of the SBS instability. Comparison of the spectra in Figure 3-4 with the growth curve for a standing wave pumped ion/electromagnetic instability (see Appendix Figure bl) shows marked sinilarities, for 
instance. The full discussion of this problem is better served in Chapter 4, however, in which the formation of localized fjeld/ density modulations is treated. Suffice it to say here, the spectrum broadens in precisely the fashion envisioned earlier in the section until the blue shifted waves reach large amplitude, and thereatter, it. changes explosively.

The energy content in the broadened spectrum is a1so interesting. To study this, however, an alternate calculational strategy was used. Instead of launching a wave with an initial, known energy density, $i$ e., $\left(\mathrm{V}_{\mathrm{os}}^{2} / \mathrm{v}_{\mathrm{tH}}^{2}\right.$ and fllowing it to deplete, the pump was initialized at a given level, and then maintained. Saturation at a steady level could only be accomplished through dissipation or scattering energy from unstable regions of $\hat{k}$-ipiace to stable ones, at a rate equal to that at which the instability coupled energy into the system. This certainly bears a resemblance to the weak turbulence picture of saturation of the parametric decay instability, though the analogy cannot be pushed too far.

Figure 3-5 shows the average energy density of electromagnetic waves in the saturated state as a function of pump intensity divided by threshold pump intensity*. This total Eield energy scaled Iinearly with the pump ratio, up to ratios of $8-10$. Even in this regime, it was qualitatively evident that the RMS fluctuations increased with

\footnotetext{
*Since the intensity is merely the enregy density in the mode times its group velocity, the pump strength ratio at a given density is the same for intensity and energy density.
} 
increasing pump strength. This is clear in Figures $3-6$ and $3-7$ which show the time history of the total energy density at $\left.\left(v_{\text {os }} / v_{\text {os }}\right)_{\text {thr }}\right)^{2}=2$ and 7 respectively. Neglecting the transient overshoot, both go to a steady state in which the total energy varies around a constant value. it the lower level, nejther the fluctuations ror the mean have any temporal structure. Nevertheless, even with the temporal avolutions at ( $\left.{ }_{\text {os }} / V_{\text {os }}\right)^{2}=7$, a recurring pattern is discernible over several thousand $\omega_{0} t$. The mean level of the fluctuations, as shown in Figure $3-5$ as a function of the pump ratin, increases roughly linearly With increasing pump strength. For pump strengths stronger than shorn in Figure 3-5, the saturation level either increases faster than linearly or a steady level is not reached at all, within the limitations of the corputational model.

To ottain a clearer picture of the temporal evolution, we have plotted the time histories of the modes surrounding the pump in Firure 3-8 for the case $\left(v_{\text {os }} / V_{\text {os }}\right)^{2}=7^{*}$. The traveling wave pump wis raintained at mode 28 , that is $\mathrm{K}_{0}=28 \frac{2 \pi}{\mathrm{L}_{\mathrm{o}}}$, fixed at a level corresponding to the mean energy density of a standing wave pump at the sime $\left(V_{o s} / V_{t h}\right)^{2}$. After rapid initial growth, the linearly most unstable wave in the system, $K=-27$, saturated at a level several timen that of the pump. It in turn immediately drove another backsiattert? way, $k=+27$, to high levels. This alternating direction astoll artinued until tha spectrum had settled down to a quasisteady

\footnotetext{
$\therefore$ fo se presise, wh plot the wavenumbers in both directions around the in, $i+\ldots$ the intervals $\left(k_{0}-3, k_{0}+2\right)$ and $\left(-k_{0}-2,-k_{0}+3\right)$.
} 
state. That state had been reached by about $u_{0} t=1500$ and contirued with enly minor changes until as $t \doteq 4000$. The exact timing of these events, of course, is not particularly relevant, since the ralculations employed an unphyslcally low electron to ion mass ratio $\left(m_{e} / M_{i}=.01\right)$. The timing of the significant phases of the spectrat evolution are mentioned only to fllustrate the relative stability of the spectrum over periods long compared with other time scales. For pumps nearer threshold, the stable phase persisted throughout the duration of the calculation $\left(\omega_{0} t=7500\right)$. For the present case, however, qualitative changes occurred after $\omega_{0} t=4000$. These again were associated with the appearance of blue shifted modes. The shorter wavelength waves saturated at amplitudes gxeater than that of the pump. Thereafter, the 1ntensity of the previously created red shifted lines are alst altered, and additfonal red lines such as $\pm\left(k_{0}-4\right)$ begin to be observable. 'Thi direct role played by the blue lines is not obvious, but it is clear in stronger pusp cases that their appearance stgnals the rapid diffusive spread of the spectrum to the longest wavelength modes allowed in the system. Again it is tempting to Identify these lines with the purely growing branch of the ion scatcering instabtlity found from linear theory. By $\omega_{0} t=4000$, though, the system is certainly nonlinear. It should be noted that the growth of the blue Ines shown in Figure $3-8$ is only approximately exponential, and any growth rate is ill-defined. Nevertheless, this is only further proof that the nonlinear state of the SBS instability is rich in wavewave interactions, which had not been previousiy discussed. 
Sumuarizing the conclusions of this section, a series of calculations were conducted in a k-space version of the reduced description model, for the express purpose st determining whether nonlinear wave interactions associated with the SBS process could influence the . spectrai characteristics. Two such mechanisms were ciearly isolated, harmonic electromagnetic wave generation and repeated scattering. The latter was able to broaden the electromagnetic wave spectrum extersively at moderately low pump strengths $\left(\mathrm{v}_{\mathrm{os}} / \mathrm{v}_{\mathrm{th}}\right)^{2}=.2-.4$. Furthermore, a powerful diffusive broadening mechanism was observed for sufficiently strong pumps, which was capable of driving waves at arbitrarily small wavenumbers. Unfortunately, it was not possible to determine what "sufficlently strong" meant in terms of real system parameters, since the system periodicity was so strongly built into the computational model. The effect of waves escaping from "the box", or any end effects in fact, could not be treated with this method. 
SECTION II

Inhomogeneous Brillouin Scattering Studies

A very suitable method for treating large scale inhomogneitius, finite system length, and end effects on the Brillouin scattering instability ras found to be, sjmply integrating the RDF equations, $(2-1)-(2-6)$, directly. Partic?e simulations might also have been useful, but the freedom from particle noise was a distinct advantage in resolving weak mode-coupling effects. Further, the reduction in computer time needed for a given case facilitated wic parameter studies that would have been prohibitively expensive with particle codes.

The first calculations treated a locally flat density profile, with the ends smoothed to reduce classical reflection. Several wavelengths of vacuum were retained on either end of the profile to facilitate the introduction or removal of electromagnetic waves. (Transmitting boundary conditions on electromagnetic waves are exact in vacuum, but only approximate in dispersive media.) The source of the waves, or "laser", was an antenna cell just within one boundary. If the pump was initiated smoothly, a wave could be grown, which propagated into the plasma. The propagation was an aspect of the scatcering problem totally neglected in the homogeneous, perlodic calculations. Strong scattering, however, at the "frcnt" of the plasma could significantly alter the instability evolution of the "interior". 
For the problem of SBS in a homogeneous, finite length system, unalytic expressions for the steady reflection coeffictent can be cerived in two Iimits. These are when the product of the ion damping rate $\frac{v_{i}}{u_{i}}$ and the interaction length, $b_{0} L$, is either much greater cr nuch less than unity. In the former case, the process is backward anplffication wile in the latter, the instability is absolute, growing exponentially at all points in space.

The expression for reflectivity when $\frac{v_{i}}{\omega_{i}}<\frac{1}{!_{0} L_{0}}$ is

$$
1=\sin \left(\theta L_{0}, C_{b}(0) / C_{f}(0)\right)
$$

where $\sin$ is a Jaccbian elliptic function, $c_{b}(0)=k_{b}^{1 / 2} A_{b}(0)$ is the normalized backtraveling anplitude at the incident bourdary, $x=C, C_{f}(0)$ the normalized forward amplitude, $i \in$. , the laser amplitude, and $\theta \approx-/ 4 k_{\text {vac }}\left(v_{o s}\left(v_{t l}\right)\left(2 n_{o} / n_{c r}\right)^{1 / 2}\right.$. Since the reflection coeffictent for SBS is essentially $\left(C_{b}(0) / C_{f}(0)\right)^{2}$, the conditions for apprectable stimulated reflection is $e_{L}>\frac{\pi}{2}$. At $\mathbf{n}_{0}=.5 n_{c r}$, this corresponds to $\left(v_{o s} / V_{t h}\right)^{2}>\left(L / h_{0}\right)^{-2}$. Since this expression is valid cnly in the short system $1 \mathrm{im} 1 \mathrm{t}$, we olay say that $L=10 \lambda_{0}$ is a typj.cal length, giving a threshold of $\left(\mathrm{v}_{\mathrm{cs}} / \mathrm{v}_{\mathrm{th}}\right)^{2}=.01$. Thls is orders of magnitude greater than the homogeneous threshold. Once the threshold is exceeded,however, the reflection very rapldly rises to unity.

The time requirec to establish the steady state is not given by (3-3) - but is related to the growth rate. Near the inhomogeneous threshold this may be longer than hydrodynamic expansion times. As 
ar example, an intensity twice the inhomogenenus threshoid is predicted to give sixty-five percent reflection. Even with a reduced ion/electron ratio $\left(m_{\mathrm{e}} / \mathrm{M}_{1}=.01\right)$, though, the periodic calculations showed that at this pump strength the time to exponentiate two crders of magnitude was roughly $\omega_{0} t=10^{4}$. Since the expression was valid only for fairly short systems, it is clear that hydrodynamic expansion will extensively alter the density prufile before the steady state is established. The effects of profile modification may in general be expected to limit the applicability of (3-3) to physically unrealizable conditions.

A series of cases were made to examine SBS in the weak dissipation/short system limit. For intensities above the inhomogeneous threshold by a significant factor (such as 10), the ion density fluctuations were observed to grow. Correlated with the increasing density fluctuations was the rise ir reflectivity. Regardless of the particular intensity, the growth of the ion waves and of the reflec.. tivity did not subside until virtually one hundred percent of the laser was being reflected. As the intensity was reduced, only the rate of growth was decreased (see Fig 3-9). Growth still continued until total reflection was established. Despite the qualitative agreement with the analytic theory, it was not possible to verify the quantitative aspects, especially near threshold where the theory needed to be tested. Even at ten times threshuld, the srowth had not saturated in typical calculational times $\left(2000-4000 \omega_{0} i\right)$. The near threshold regime, though, has little applicability in plasmas because 
the expansion time is comparable to the growth time. In the stronger pump cases, the calculations were consistent with the theory. The other limit, $\frac{v_{i}}{w_{i}}>\frac{1}{k_{o} L_{0}}$, is easily oblained for $k_{0} l \geq 10$ when $\theta_{e}$ is not too much greater than $\theta_{1}$. An analytic expression for the steady state reflection is also derivable in this case, (c.f. Kruer $^{21}$ )

$$
G_{b}(x)^{2}=C_{b}(0)^{2}\left(1-R /\left(\exp \left(\phi_{x}\right)-R\right)\right)
$$

where $\emptyset=1 / 4\left(m_{\mathrm{e}} \mathrm{M}_{i}\right)\left(\mathrm{V}_{\text {os }} / \mathrm{c}\right)^{2} \omega_{\mathrm{p}}^{2}\left(\omega_{i} \mathrm{v}_{i}\left(\mathrm{k}_{2}{ }^{2} /\left(\mathrm{k}_{2}-\mathrm{k}_{0}\right)\right)\right)(1-\mathrm{R})$. This expression predicts exponential amplification of a backscattered signal until it reaches the front edge of the plasma. Given an initial tntensity, $c_{b}\left(x_{o}\right)^{2}$, the steady state reflection can bc computer without excessive difficulty. Numerical calculatiors were conducted in a finite slab plasma to test the theory. For $v_{i} / w_{i} \doteq \frac{1}{4}$ the backward traveling signal does indeed increase exponentially as it propagates through the slab as shown in Figure 3-10. The observed gain coeffictent is a function of time, though. IL is smaller than $\emptyset$ during a transient phase of the instability, but rises toward il as a limiting value.

In the numerical model, the initial backscattered signa]. is due to classical reflection from the back edge of the plasma. For a given profile, the fraction reflected is fixed, so that $c_{b}(L)^{2} / C_{f}(0)^{2}$ is a constant, depending only on the profile shape. Equation (3-4) evaluated at $x=I$, dividied by $\mathrm{C}_{f}(0)^{2}$ shoujd also be a constant then. 
Within the accuracy with which the "steady" reflection level may be measured on the plots $(<5 \%)^{*}$, this is found to be the case. It should be mentioned that this "steady" level has only limited significance, since hydrodynamic expansion or the slab had created a shaj.10w ramp in front of the homogenevus region after about $\omega_{0} t=10^{3}$. Although the expansion was Enhanced by the reduced mass ratio, cases lasting between $2-4 \times 10^{3} \omega_{0} t$ are still very short compared to other laser fusior time scales. The pre-ramp could be expected to alter the "steady" state reflection, since strong SBS was evident in the ramp after a few thousand $\omega_{0} t$.

The early analytic theories, from which (3-3) and (3-4) were derived, were motivated by scattoring in crystalline media. Unfortunately, the static conditions of solid-state materials are nut obtainable in short-pulse laser flasmas. So long as the finite profile did not change much from its initial configuration, the calculations of heavily ion wave damped SBS were nevertheless in excellent agreement with the analytic results. Plasma expansion and profile modification Introduced facturs not inciuded in the theory, however, after which the computational results could diverge significantly from that predicted.

Harmonic satellite generation and repeated scattering were strongly modified in the finite slab geometry. For pump powers only

\footnotetext{
* To avoid ambiguity, note that the five percent uncertainty applies to the measurement of any reflection level, not that a noise level of five percent was present.
} 
slightly above the inhomogereous threshold ( $<<20 \%$ ) only the narrow S3S line is observed in the reflected spectrum, and only the pump line in the transmitted. Wher the pump is increased to the level of strong scattering ( $R \geq 50 \%$ ), much temporal evolution is observed. As an exampie, for $\left(\mathrm{V}_{\mathrm{os}} / \mathrm{v}_{\mathrm{th}}\right)^{2}=.81, \mathrm{n}_{\mathrm{o}}=.85 \mathrm{n}_{\mathrm{cr}}$, and $\mathrm{L}=10-14 \lambda_{\mathrm{o}}$, the average reflection coefficient reached seventy percent after $w_{0} t=2 \times 10^{3}$. Thereastel successive downshifted spectral lines appeared alternatively in the forward and backward dirsctions, clearly to 3rd order in the backward direction and 2nd in the forward. Figure 3-11 shows these spectrat at roughly their maximum width. Both the relative frequency shifts and the relative intensities are consistent with a repeated scattering model. Somethat later, the primary scattering had reached sufficient magnitude for pump depletion to occur. The reason this terminates re-scattering is that the backward wave is exponentially amplified urder these conditions. Mlthough exponentiation also persists throughout the transient phase of the instability, the rate is small enough that an intense backscattered signal has a long irteraction pat: in the plasm: The interaction length continualiy decroases as the process approaches the steady state, however. The net result is that the strong scatcering near the front "starves" the rescattering process, i e., there is ittle field over the full interaction path in the forward direction. Since the analytic theory predicts strong SBS when $\emptyset \mathrm{L}_{\mathrm{o}} \simeq 5-10$, ve expect this inhibition of repeated scattering to occur at even lower 
intensities in actual laser plasma experiments than is seen in he relatively short computational systems.

Lnder sirong SBS cunditions, harmonic generation mas also easily observabie. Spatial Fourier analysis of the field and dersity within the plasna ( $k$-spectrum) showed $3 K_{0}$ and $5 k_{0}^{*}$ harmonic content in the field and harmorics up to 5 th order in the density fluctuations. None of these lines appeared outside the plasma, though, as is expected from a forced oscillation. The hamonics of the high frequency wave are nonpropagating modes. They may contribute to the internal mode-coupling dynanics, or they may contain a significant fraction of the electromagnetic energy in the plasma, but they are not part of the measured spectra in laser plasna experimenti.

Calculations were also performed in inhomogeneous, linear density gradient plasmas. In light of the fact that rescattering is an inherently front/back symmetric process, it seems even less propitious in a density gradient than was seen before. Nevertheless, a marginal amount of rescattering was observed for strong scatteri:a cases. This was only transient, and so seems an unlikely candidate for experimental measurement. Higher parmonics of tha primary density wave were also seen, but the electromagnetic hamonics were relatively weaker, and again even the small $3 k_{0}$ concent was not manifested in any frequency components.

On the basis of these one-dimensional slab studies, certain conclusions can $b \geq$ drann. The analytic expressions for siany state SBS reflection, in the highly damped and highly undamped lirits, were 
consistent with the caiculations. Oniv indirect evidance was availahlo in the undamped case, but the general features of the highly dumped calculations agreed very reasonabiy with the analytic expression. These provide confidence in extrapolating a limited number of numizial results to a mort general parameter space. Fourier harmonies of the field were generated in the plasma interfor but were Found to be nonpropagiting. Re-scattering, on the other hand, clearly broadened the scattered (and transaitted) spectra during early phases of strong SBS. Pump idepletior tveatualiy destroyed this process. This late time behavior was preciselv the information we sought with the reduced plasma description. A poientially important process such as stimulated rescattering was shown to be throttled in the selfconsistent evolution of Briliouin backscattering.

These results do not rule out mode-coupling as a spectral broadening merhanism, iut they do mitigate against its importance in stimulated Rrillouin backscattering. The stinulated scattering is not intrinsically one-dimensional, as studied above, and it is clear that scatiaring in otler directions cas behave qualitatively differently. sidescattering, for instance, doplates the laser strongly in its orifinal trajectory, while purping uniformly along that of the scattered photons. Furtiermo'e, in an inhomogeneous plasma, refractory losses in the sidescatced direction tend to filter out ald but a narrow rone of anglos for effertive gain. Without pump depletion, rescatering can prosper. With a natural diretional salector, the 


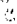

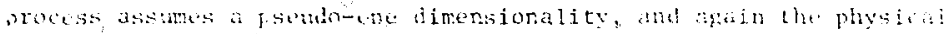

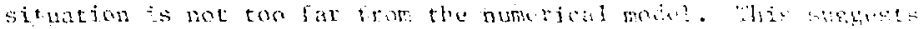

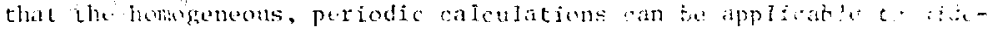
soitterignt 


\section{SECTION III}

\section{Appliration of Itahomogeneous Calculations te a} Scattering Experiment Performed at NRL

The homogeneous and inhomogeneous simulations of stimulated Brillouin scattering performed in the previous sections will now be applied to a typical scattering experiment to illustrate their utility. The experiment at NRL ${ }^{15}$ is espectally interesting since measurements ac difterent angles of laser incidence led to diff-rent backscattered spectra. The published measurements are therefore reproduced in Figure 3-12: Although the total energy collected at each argular position was about the same, the spectral characteristics varied considerably. Application of the results of the previous sections yialds a qualitative explanation for this varfation. The spectra measured for normal incidence was remarkably narrow. This was tentatively identified as stimulated Brillouin backscatter, and that hypothesis seems reasonable. The density scale length was estimated to be about 20 microns in this Nd-laser experiment. This is comparable with scales used in the inhomogeneous RDF calculations. In those, strong SBS was observed for field energy commensurate with the themal energy of the plasma. The laser power reported for the experiments seems wficient to easily satisfy these intensity requirements. Iittle spectral brodening was observed in the calculations, however, despite even strongly occurring SBS. This is consistent with the backscatter measurements. 
At the intermediate angular position $\left(\theta=24^{\circ}\right)$, some broadening of the spectrum was measured. It was an irregular spectrum, though, consisting of many distinguishable lines. Analysis of this measurement would involve too many details, which would tend to distract from the main points. We will thus proceed to the measurement at the largest angle.

At the $45^{\circ}$ position, the spectrum possesses a nearly continuous spectrum, albeit with structure, asymetrically broadened toward longer wavelengths. The total spectral width at this angle is an order of magnitude wider than the one measured at normal incidence. This type of spectra is similar to the calculated ones in which repeated scattering dominated. Although the focal lens could have collected some directly backscattered SBS, this source is no more likely to lead to broadening than it did for normal incidence. At the laser power of the experiment, however, Brillouin sidescatter is apparently above the inhomogeneous threshold ${ }^{22}$. These waves would presumably be generated parallel, to the density surface, but would then refract out of the plasma at some other angle. Since sidescattering is inherently multidimensional, the calculations in this chapter do not apply to the process directly. The magnitude of sidescatter can be estimated, though, from knowledge of experimental parameters and inhomogeneous theory. The trajectory of a wave can, furthermore, be calculated as a function of initial density and angular orientation. From this, one can infer coherence lengths for subsequent SBS backscatter ${ }^{23}$. 
The likelihood that repeate scattering is responsible for broadening at a given angle relative to the target nozmal can be evaluated. The cetai's of chis aralysis unfortumately are tedicus, and so have ticen fontponed until Appcnitix E. The key points, nevertheiess, can be brifoly sumarized. At the intensity reported in the MnL experinent, Loth Pri]louin back- at sicecatter are far above theoretical thresholds. For typical scale lengths, the coherence lengths were long encught for siciescattered waves cf reasonable magnitude to repeatedy tackscatter. The absence of broadening in the roxal direction, only sporadic broidening at $24^{\circ}$, and pronounced broadening at $45^{\circ}$ is consistent with the cccurrence of strong siclescatter, but only below about $n_{0}=.5 n_{c^{2}}$. Although djscrete lines ware not evident in the list spectra, there is apparently some structure at $10 \AA$ spacing. Assuring linear ojspersion relations and a hydrogenic plasmä, however, a ciscrete specing of $20-25 \hat{A}$ is experted. There are several possible explanations for the rieasured spacing which would nake them consistent with repeated SBS backscatter. Nonlinear frequency shifts coujd cause the frequency decreases to be lower than linear theory predicts. Wternatively, the Brillouin shift in the polynthylene targets could be more typical of carbonic tather than hycrogenic plasmas. This alone would account fol the observed spacing. Finally, although polyethylere, aluminum, and copper target ratexials were used in the experiments, the paper does net specify which type the broutener spectra examie was iron:. The discrepancy, moreover, is mly on order 2, so that repeaced srattering is not stonely arglied agatust on that basis. 


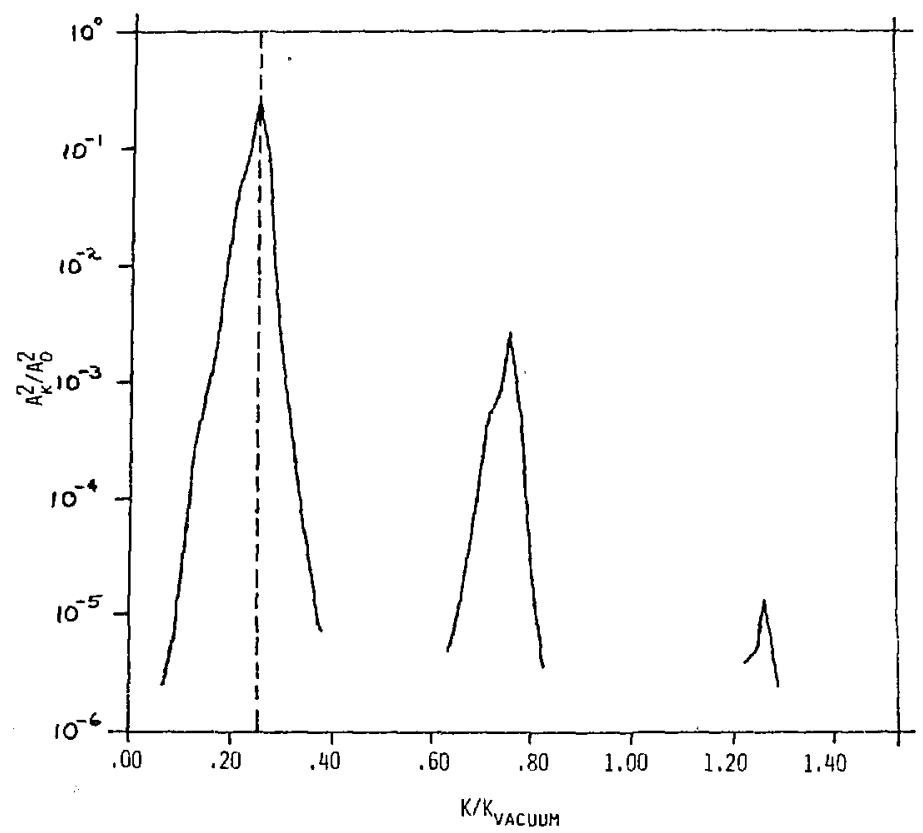

Figure 3-1 Electromagnetio field spectrum at $\omega_{0} t=1400$, pump depletion allowed, $n_{0}=.935 \mathrm{n}_{\mathrm{g}}$, L=40 $\lambda_{0}$, $\theta_{\mathrm{e}} / \theta_{1}=100, \quad \mathrm{~m}_{\mathrm{e}} / \mathrm{M}_{1}=0.01 . \quad\left(\mathrm{V}_{0 \mathrm{~s}} / \mathrm{v}_{\mathrm{th}}\right)^{2}=0.25$. 


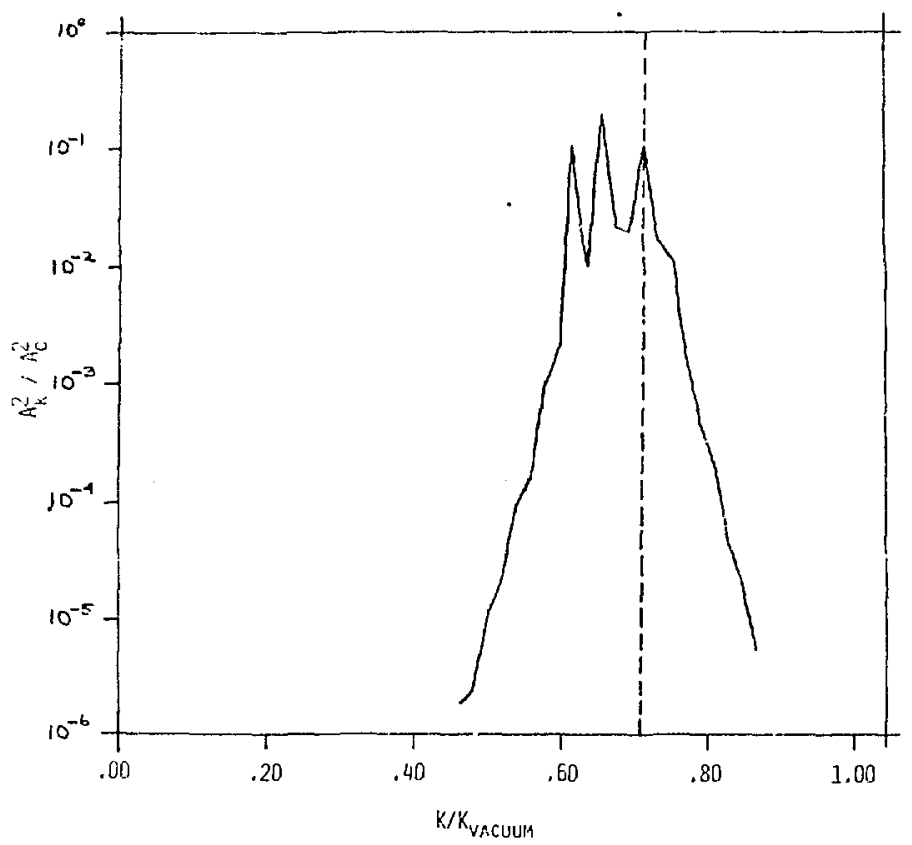

Finure $3-2$ Electromagnetic field spectrum at $w_{0} t=2800$., pump depletion allowed, $n_{0}=.5 n_{\mathrm{er}} \mathrm{l}=40 \lambda_{0}$. $H_{t:} / H_{i}=100, \quad m_{e} / M_{i}=0.01, \quad\left(v_{o s} / v_{t h}\right)^{2}=0.25$. 


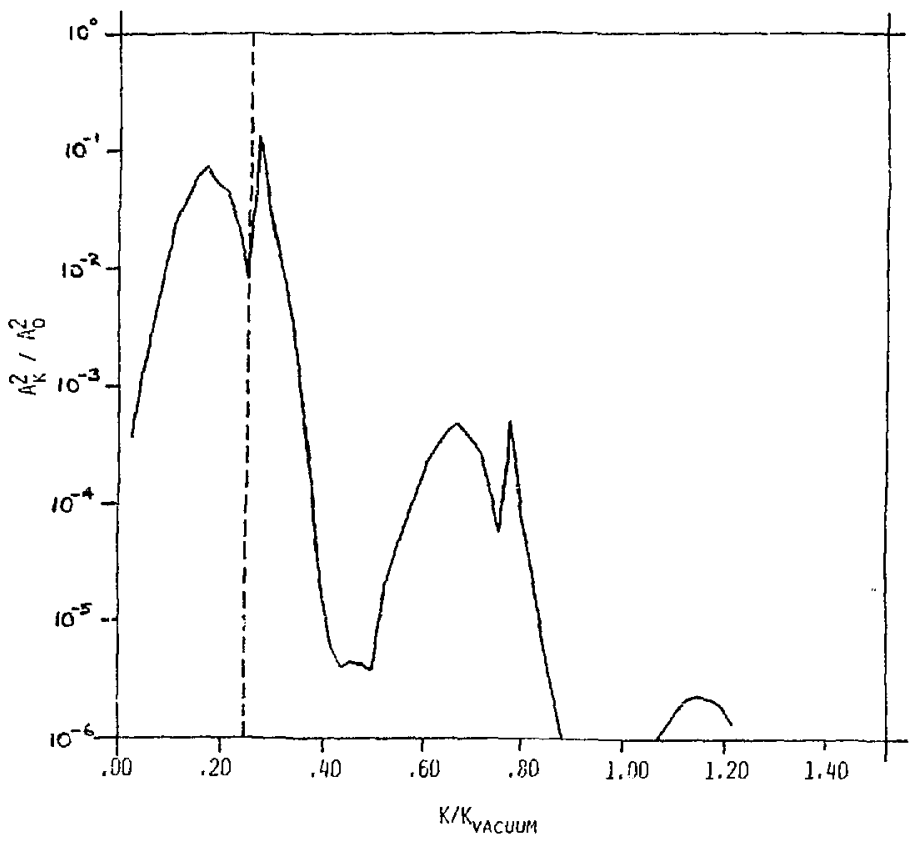

Figure 3-3 Electromagnetic field spectrum at $\omega_{0} t=1800$., same calculation as Figure $3-1$. 


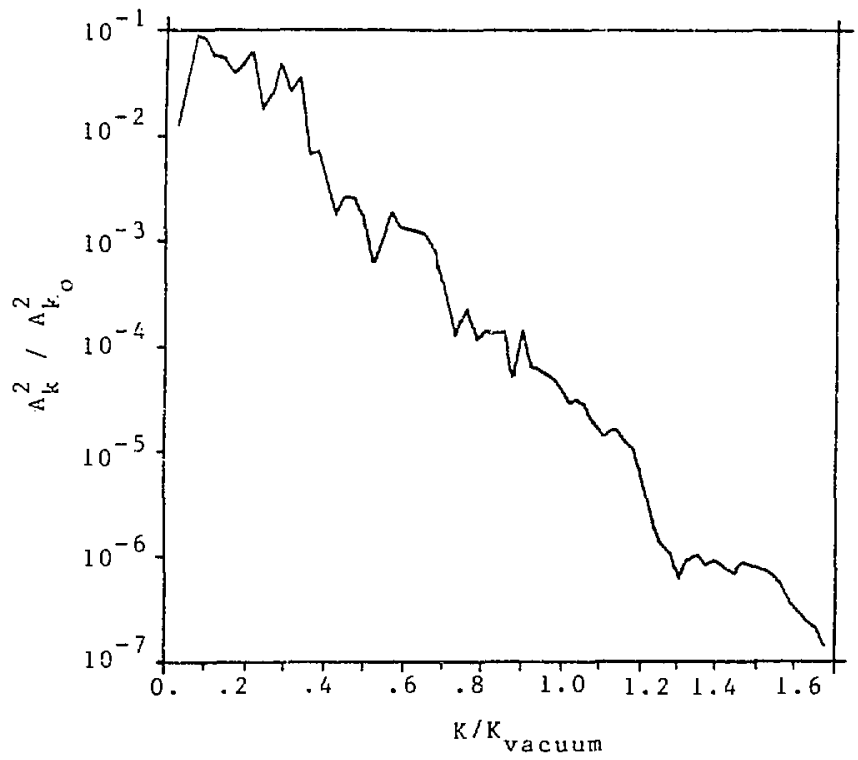

Figure 3-4 Electromagnetic field spectrum at $w_{0} t=2400$, same calculation as in Figure 3-1. 


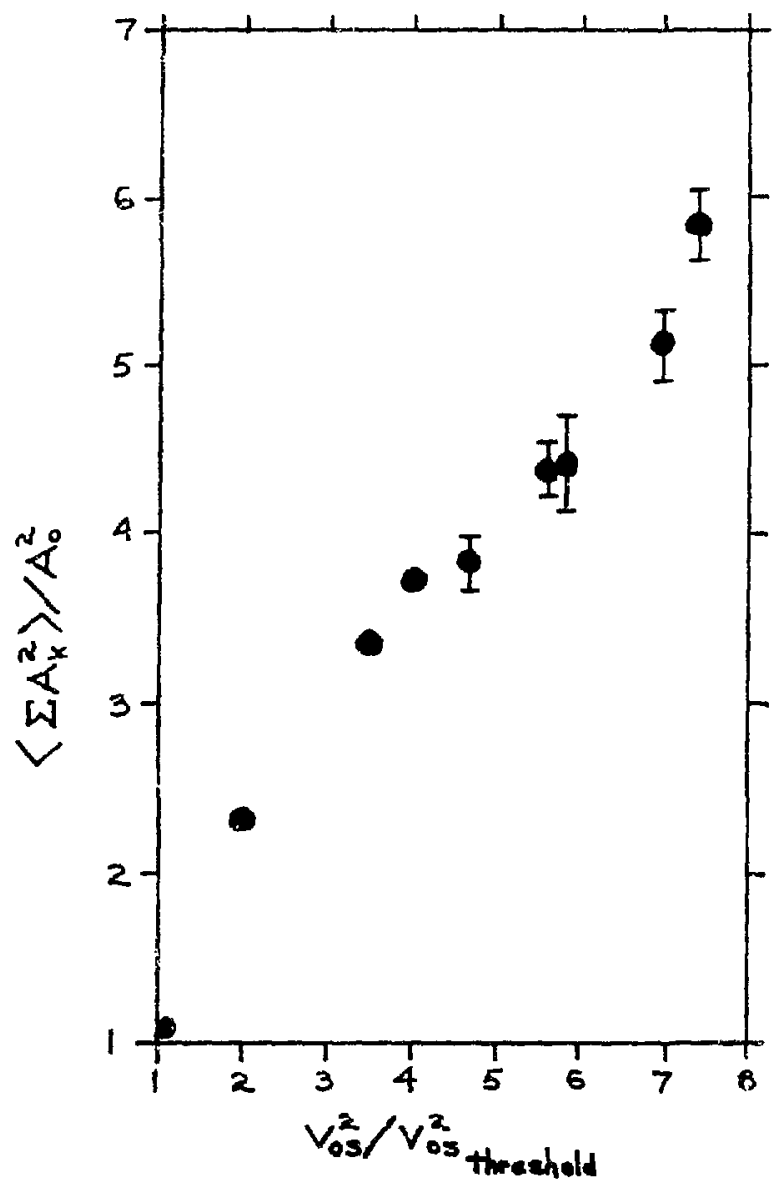

FIGURE 3-5 Averaged fleld energy density in the saturated state as a function of pump strength diyided by threshold pump strength, (Vos os toreshold 


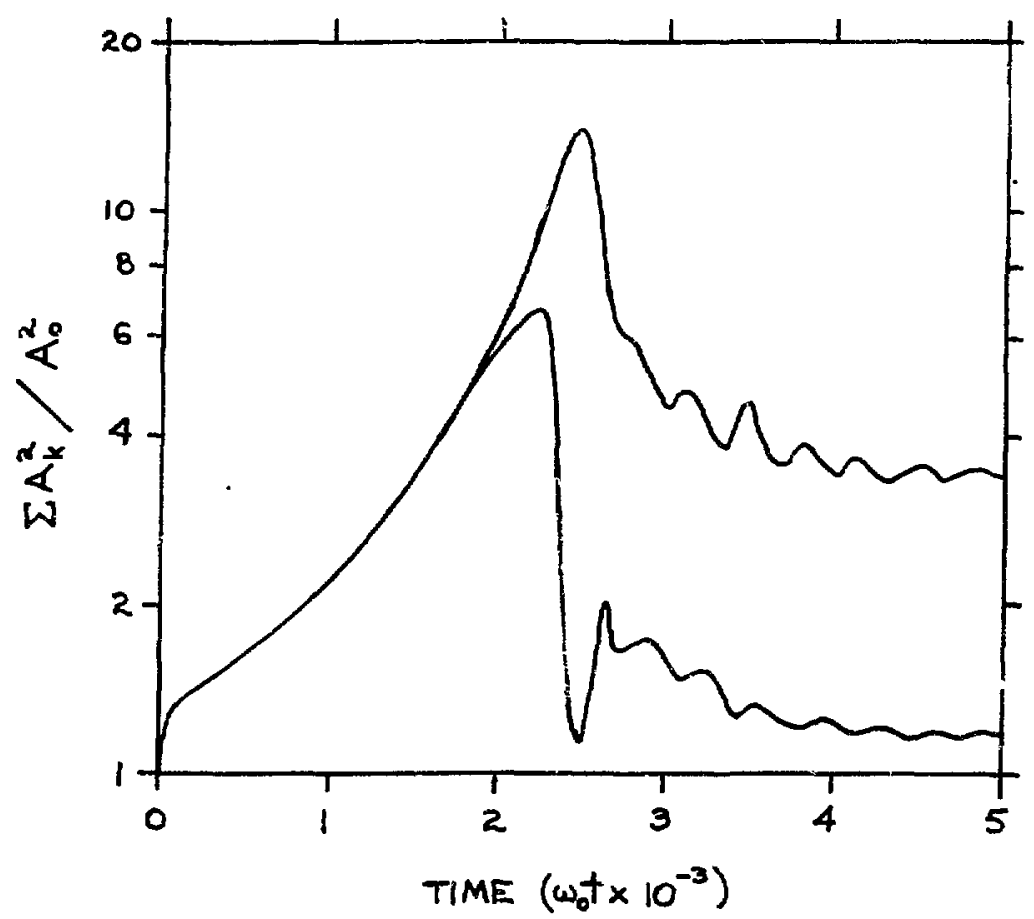

FIGURE 3-6 Time history of the field energy density, $\left(v_{o s} / V_{o s}\right)^{2}=2 \ldots, m_{e} / M_{i}=0.01, \quad L=40 \lambda_{0}, n_{0} / n_{c r}=.75$, fixed maghreshold 
69

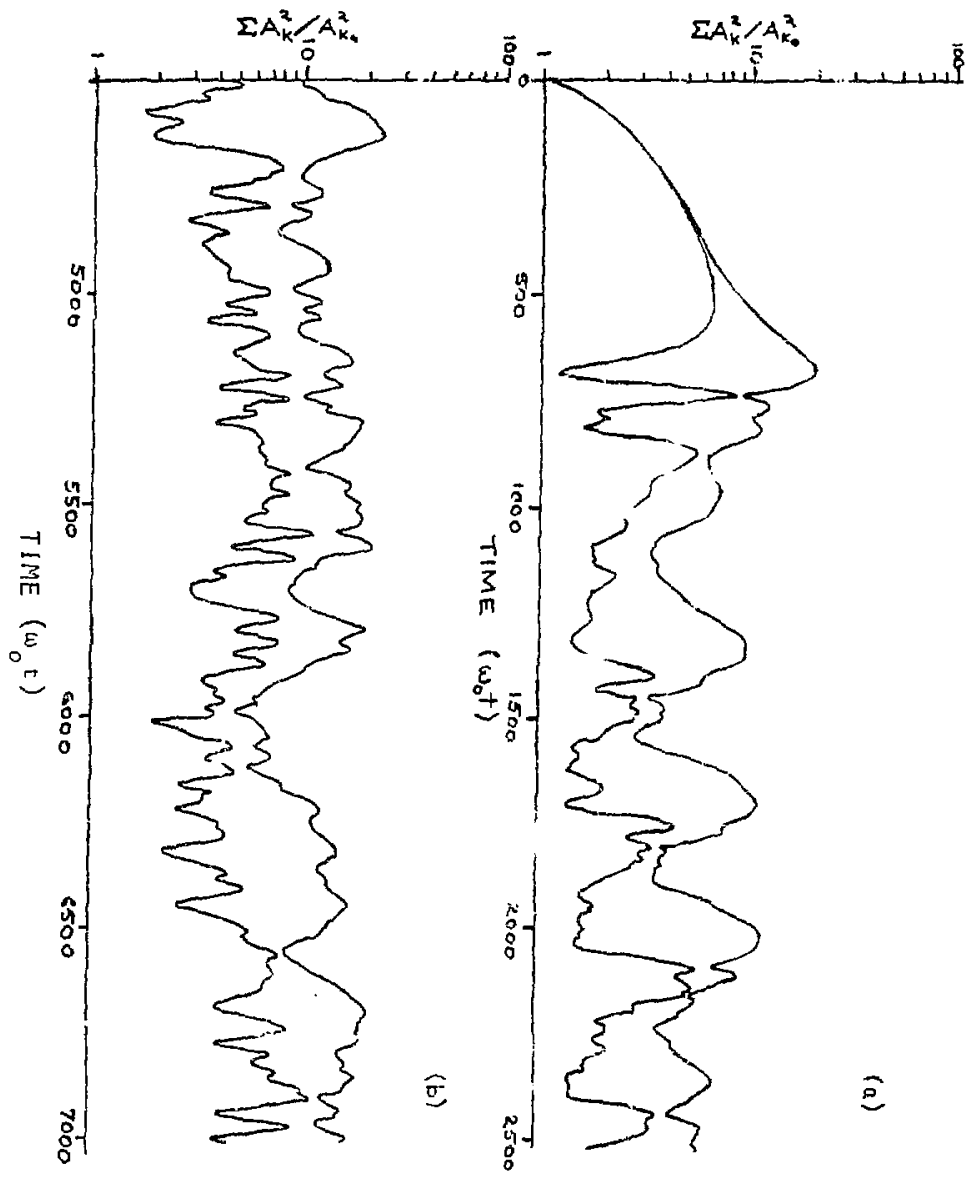

Figure 3-7 Time history. of the total field energy density, fixed, traveling wave pump, $\Omega_{0}=.75 n_{\mathrm{cr}}, L=40 \lambda_{\mathrm{G}}$. $\left(v_{\text {os }} / v_{\text {os }} \text { thresh }\right)^{2}=7$. 

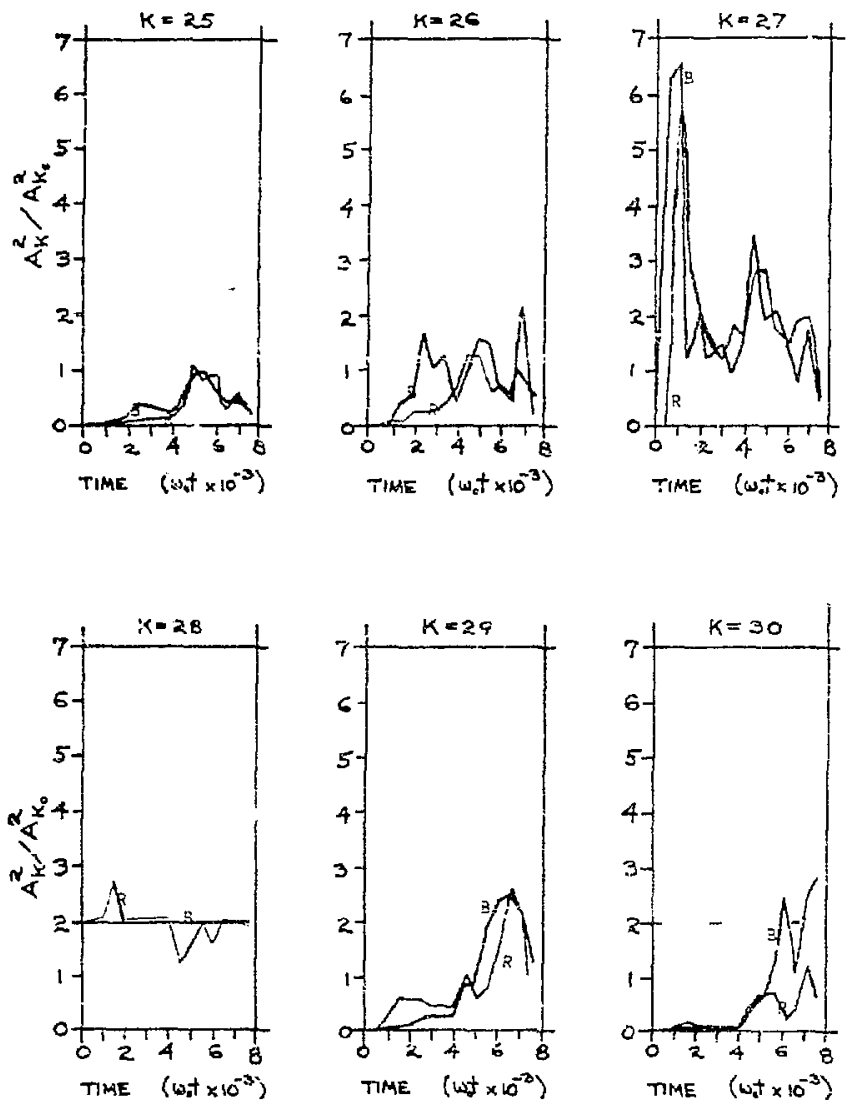

Fizure 3-8 Time histories of modes near the wavenumber of the pump, $\mathrm{K}=+28, \mathrm{n}_{\mathrm{o}}=.75 \mathrm{n}_{\mathrm{cr}}, \mathrm{L}=40 \lambda_{\mathrm{o}}$,

(vos $/ v$ s $)^{2}=7$. Waves in the + direction are labeled whthsh "B", while -. dirertion waves are labeled with "R". 


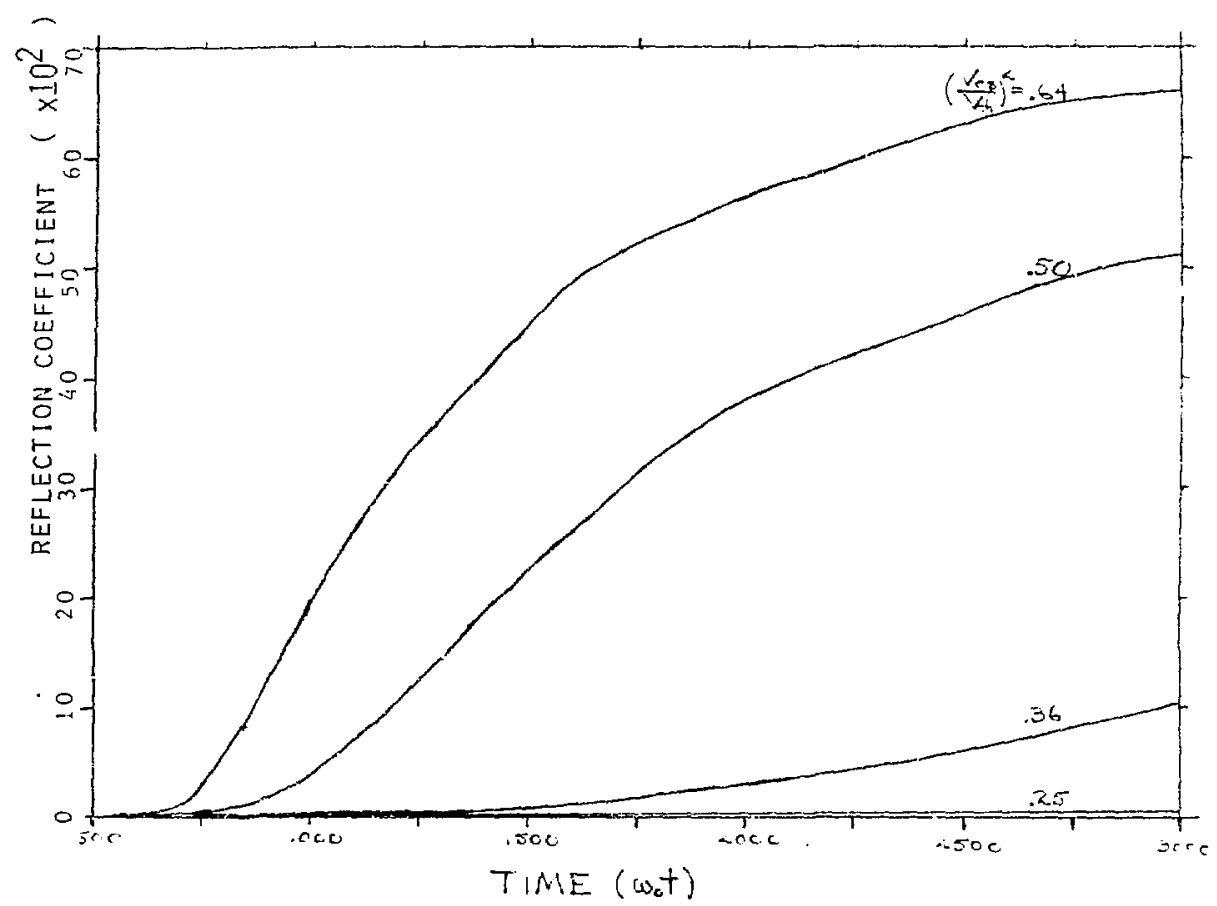

Figure 3-9 Reflection coeficient from a finite slab as a Eunction of time, for various field strengths, $n_{0}=.5 \mathrm{n} c \mathrm{r}$,
$\mathrm{L}=\mathrm{l} 1 \lambda_{0}, \theta_{\mathrm{e}}=5 \mathrm{keV}, v_{i}=0$. 

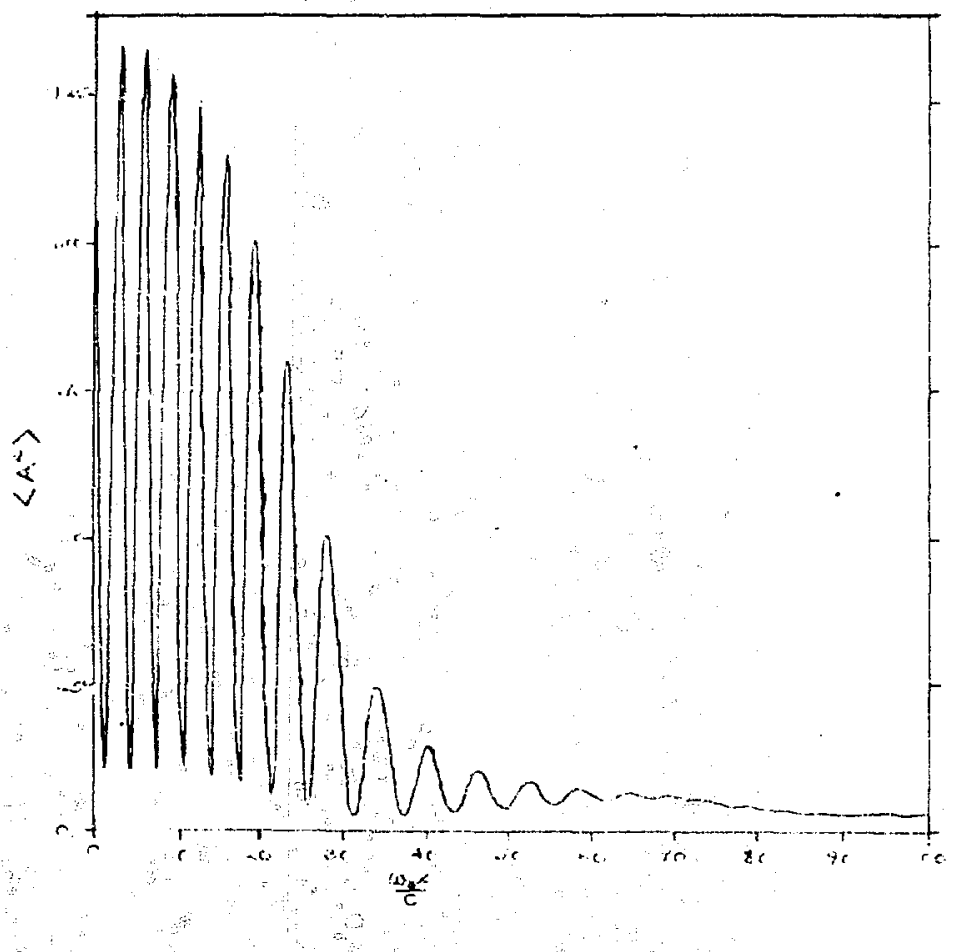

$\mathrm{s}$

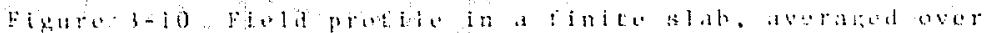

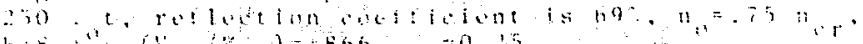

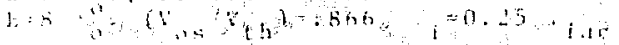


$\overrightarrow{3}$
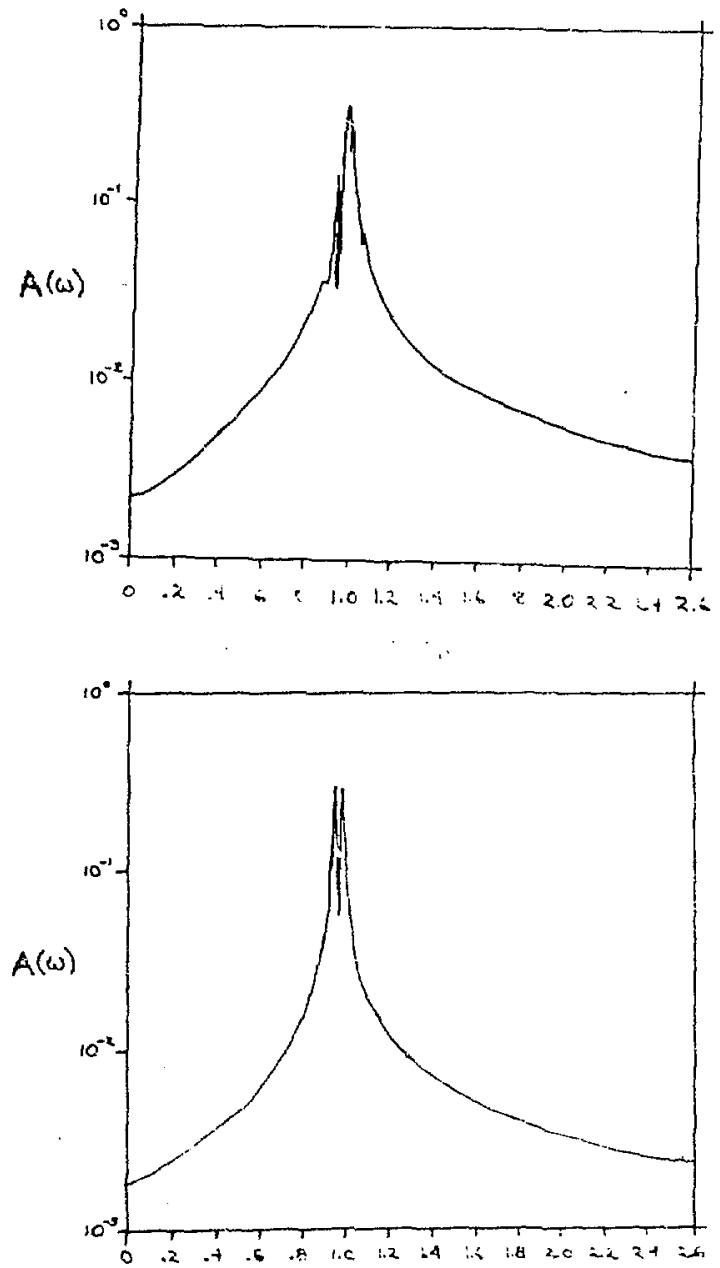

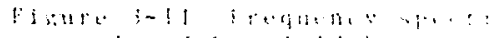

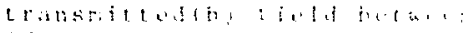

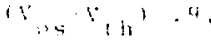

$n_{1}, 4+3 t-11 !$. 


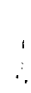

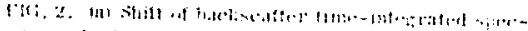
t

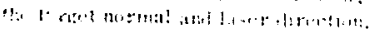

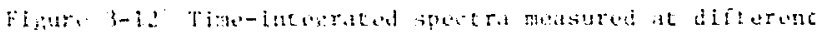

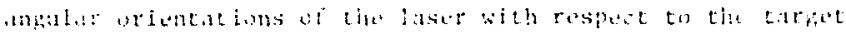
i.ting in: 13$)$. 


$$
\text { Cr: '.'TR } 4
$$

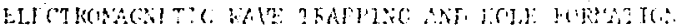

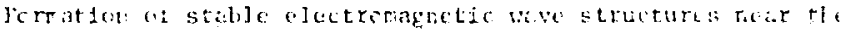

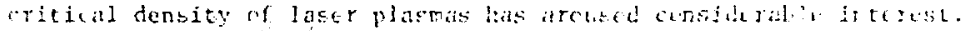

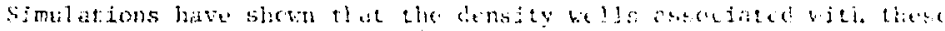

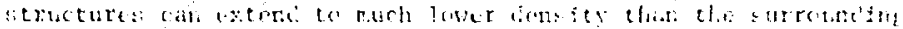

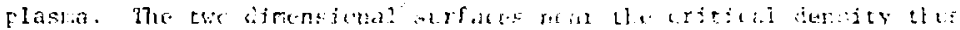

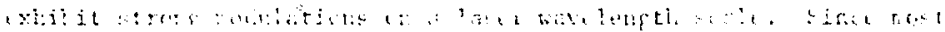

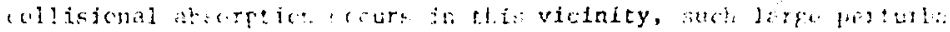

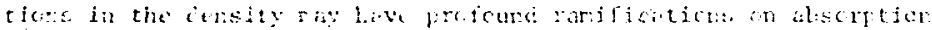

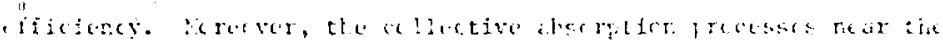

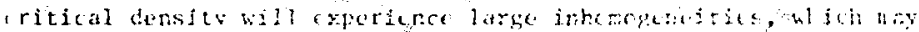

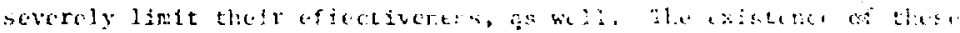

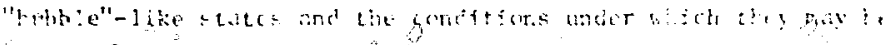

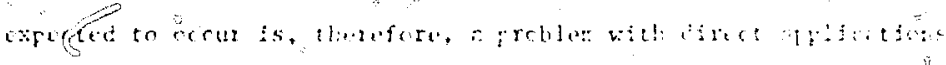

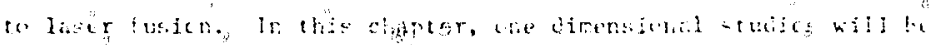

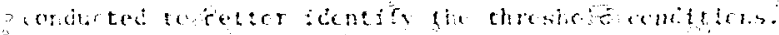

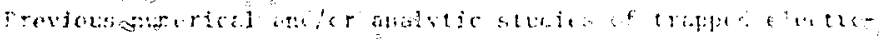

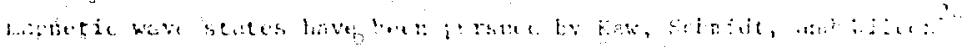

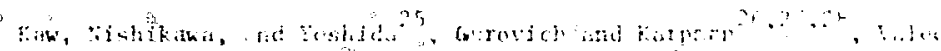

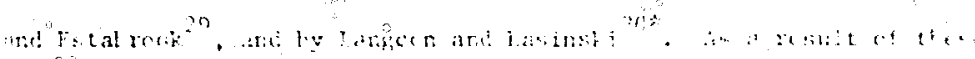

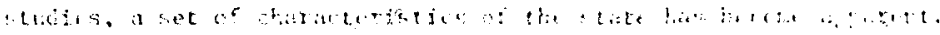

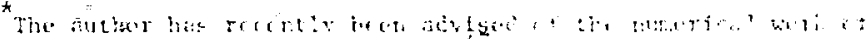

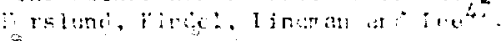


dif blbbles ete only cterved reasonally near the critical surface. A slat, or "sanrlkich" state isfound to be unstable to the growth of transverse perturbatens, of a sausage type. The bounded ellipsoidal otates that result from the break-uf suggest that the three dimenSLmal structures iculd approximate an olectromignetic bubble inbedied in the plasma. Cnce formed these are cbserved to be rejatively stable cver tiousinds of laser periods. This does not 1 mply: that they do rot affect rither processus, only that they do not partake disectly.

Al thcugh the work cited above has gone fin toward understanding the qualitativa nature of the trapped fave state, iftele wort: has been drected trward defining threshold conditions. It is true that whe phenomena orius only near the critical censity, but how near is net clear. Also, there are several ressons to believe that the field intensity tust te of urier $\left(\mathrm{v}_{\mathrm{es}} / \mathrm{N}_{\mathrm{t}}\right)^{2}-1$, but it would be useful to Gnow if the we cenditions thier which the state rould form under oss strongly gromencitions. In this chapter, the inproved understancinfest the flienomena dorfvec iror numerical stedies has per-

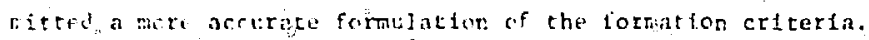
a!j the work in Wis clapter wil te limiled to one dimedston. oven throph ibe slab state is uretabie, the one-dimersional calcula-

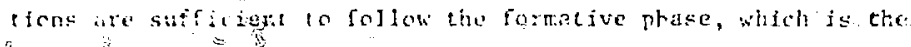

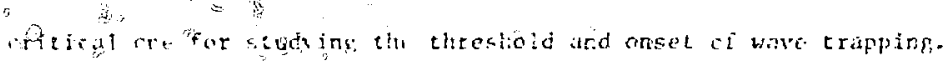

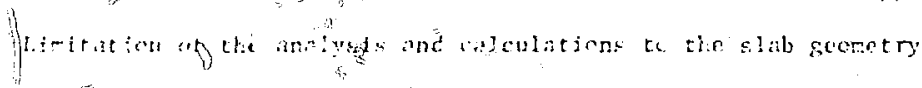

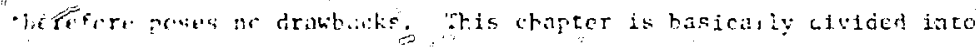


three parts (as is all gali). In the first part, nuderical and: analyt1c studies of wave trapping are performed in hom tercous dersit" profiles. These calculations are described firsit. In the process it analyzing these, several key concepts relative to the wave trappi. Fhenomena emerged. Anong these are that the nagnitucie of the . . . tivg. wave component is the stgniffcaut fraraeter and tiat paraitet it instabilities play no more than a seconcary role. A sjnplo r... i cn a cubic nonlinearity is very helpful in understanding the: tions. The understancing gained frem the homogenecus studies : : : applied to more realistic inhomogeneous plasnas ir the $64 n$ : ion. Wave trapping still occurred in Ifnear Eradient density frofice, lut only close to the critical surface. The models derlved for lomofirus plasmas were found to be eastly sodified for , t in inhomgentous plasmas. In paxticular, the midels provide a tear uncergiuriag of the effect of laser bandridth on the formation threshold. Firally, the results of the first two sections are discu: in light of previous fork, and in the erfect they fudicate "bubbles" have in actuai laser fusion schemes.

Befnre proceeding, it is wortl no. ing that both particle end riv sinulations were enplcyed ir. Llis work. The two techntques agree remarkably well on threshold and earjy dyromice of the wave-trapping, process. In fact, they were very complementary. The fDF calculations were free from portede noise and fast enough to concuct partatio studies. They showed a tenciencs to tecome wastoble, tockever, whet: larpe gradento wert present. The particie simulations rin the other 
fanc renuiles a nuch targer allocation of resources, but thes.r insensitivity to Jarse sradients and kinetic non]inearities permitted alcelatior into the fuljy ronlinter state. The RTP inability to

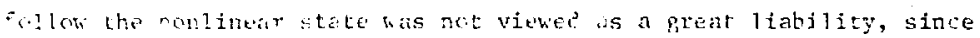
the rate tip. Whavior is intrinsiraly nultidfmersional, and since Whe forratice phase was the poiraty conern here. 
SECTION I

WAVE TRAPPING/HOLE FOWITION IN A HONOSLNEUIS PLASMA

A typical homogeneous plasnia calculation was conducted on a denstty profile such as may be seen in Figure 4-1. A vacuum space on efther side of the profile laciltated both expinsion of the prori?e and propagation of the electronegnetic kives. The pthy wes fintroduced as a traveling wave on the left toundary of the sintulation. When the wave was incident on the plasma with suffelenf intensity, censity fluctuations could be coupled with the elect:oragretic rield. Stimulated Brfllouln scattering is a fanilfar example of such coupling. Brillouin scatering was in fact observed over a vide ronfe of censities. Within roughly ten percent of the critical censity, hicrever, a qualitatively different type cf coupling cculd also be observed. Insteac of backscattering, the energy, the electronagretic fleld coalesced into highly localized structures, accompanied by déep density wells. 'The well could extenc to virtuclly vacuum at the bottom, w1th the expelled plasnia accunulating as cverderse walls. The field was initlally seen to possess nnly a local raxinumi: that is. the localized field had no nodes. Although such a strone vacuun state lasted with few qualitative changes for thausands of cycles, it dic evolve slowly, broadening the hole width. Thls is clearly seen in Figures 4-2 and 4-3. In Figure 4-2 the lenste prefile at $u_{0} t=500$ shows several density cavities just after the holeforration; ffer $\omega_{e} t 2000$, however. Figdre $4-3$ shows that the wirth has expoyded 
Arenatically. The field at the later time hes at least one noce within the cavity. The evolution from symmetric to antisjumetric state suggests a picture of ctomic ground and excited state wave funclicrs. The simulation, tholigh, showed a continuous evolution, as opposed to an abrupt jump, so the analegy should pertiaps not be overlabored. One of the more fascinating characteristics of this late time evolution was that the frequency of the trapped field decreased From $w_{0}$ to about $5-.6 h_{0}$. This is not germaine to the preseri. purposes, unfortunately, and so will not be further discussed. The incerested reader may find moxe information on this phenomena in Langdion and Lasinsk1 ${ }^{30}$.

The density regime in which the hole-forming dominated was not precisely specifled above, but a falrly shatp density separation tetween SBS and this process nevertheless was observed. As an example, two simulations, each 10 vacuuru wavelengths $1 \mathrm{mg}$, were conducter. loth were isradiated by a lasar of strength" $\left(v_{o s} / v_{t h}\right)^{2}=.81$ and the dissipation was the sane in both cases. Tigure $4-4$ shows, however. that the calculation at $r_{0}=.95 \mathrm{n}_{\mathrm{cr}}$ had developed nultiple vacuum holes by $L_{0} t=1200$. The other simulation, at $n_{0}=.85 n_{c r}$ is shown at the same the ta Figure 4-5. Strong sBs is observed, but there is r. tendency cither ac this tire or later for the fields to collapse into nonpropagating fensity welis. This is confirmed in both Wh and iarticlo simblators, eten when the intensity was strong enough of irciscats seventy to eighty percer nf the laser energy. In the parti, it irmatirns, serh strong scatering lod to density veves in 
which $\left(\frac{m}{n_{0}}\right)^{2} \sim .1$. This resulted in particle trapping and, thus, enhanced danping of that wave. The RDF calculation could not treat particle trapping, so the density fluctuations grew to physically unrealistic levels. It is significant, then, that even when the ion acoustic fluctuations were far in excess of the critical density, no wave trapping occurred at $\mathrm{n}_{\mathrm{o}}=.85 \mathrm{n}_{\mathrm{cr}}$. Thus, it can be Inferred that the simple presence of overdense density fluctuations is not sufficient in itself to guarantee hole formation.

Between $n_{0}=.95 \mathrm{n}_{\mathrm{cr}}$ and $.85 \mathrm{n}_{\mathrm{cr}}$ there 1 admitted1y a transitional regime, so the boundary between scattering ans trapping is not mathenatically sharp. The fntermediate density, however, is very useful for 113ustratiag the effect system length has on wave-trapping threshold. Becguse trapping is only marginal in this regime, one can see the wak effect due to system length. Two simulations were conducted at $n_{0}=.90 n_{c r}$, to study system length effects. Cne was 10 vacutur wavelengths long, the orher 20 . The fleld strength was chosen to be just above threshold for hole formation. In both cases, holes formed initlally. In the shorter system, however, the varuum depreasion eventually lost its field and the surrounding plasma expanded in to fill the yold. The longer systen not only exhlbited multiple holes, an Index of having far exceeded the threshold, but persisted strongly throughout the simulation duration, to $b_{0}=3000$. Altheugh 1 t can be inferred from this that system length telias docrease the wave trapping threshold, later analysis of the phenomena will show that the 
effect is actually due to the strength of the standing wave component present at a giver density.

The simultaneous localization of the field and density fluctuations suggest that the two are soupled. Brfllouln scattering is cne example of just such an interaction. The first order ponderometive potentlal couples with an ion acoustic wave there. For an fon acoustic wave one-half the wavelength of the punp wave, the potentfal and density perturbations move in priase. This resonance leads to expenential frowth of both at the expense of the pump. Given this known interaction, it is seductive to lock for another resonant Instability which would result in the localization. As the American College Dictionary defines "seduce", however, 1 e. to lead astray, analysis of the coupled wave equations dces not give any instability other than the SBS near the crftical density for a traveling wave. The analysis itself is rather lengthy and since it is not original, it has been Included as Appendix B. An example of the numerically dertved real anc: Imaginary freüuency components for a traveling wave pump is shown in Figure 4-6. Thys is quite significant since BDF calculations which exployed only the solution of those equations in real space produced waye sapplng in agreement with the other numerfcal techniques. Although difficult to rigorously prove, the qualitative differences between wave trapping and scattering indicate that SBS is not the mectanismi responsible for the localization. Since a triveling wave, furthermore, possesses no gradients In its time-averasied potential, there are no zero order forces which could give rise to the phenomenon. 
A standing wave pump leads to qualitatively different results. s.

First, zero order forces exist in this mode. These are periodic with twice the wave number of the electromagnetic wave, and could be expected to produce a density ripple with the same wavelength. coupled wave stability analysis around the homogeneous state moreover yields a different set of dispersion curves than a traveling wave, as play be seen by comparing Figures $4-6$ and $4-7$. The unstable waves are divided Into a branch with $k<k_{0}$, which possessas the same properties as SBS, and one at $k>k_{0}$. This latter 1s a parametric coupling of a purely growing density fluctuation to an electromagnetic disturbance at the pump frequency. The branches are analogous to the parametric decay/oscillating tho stream instabll1ttes which occur transverse to the iaser direction. In the presence of the standing wave, the plasma will also respond, to a zero-order furce with periodictty of $2 \mathrm{k}$. The instability predicted in che homogeneous plasma must conpete with andior be modiffed by the density ripples produced by the zero order force. Since the instability cccurs infirst order, 1t seems likely that the zero order force will dominate the system initially. The unstable waves though may still play a significant role in the Incalization process.

To clarlfy the relative importance of these two autonomous density perturbtations, coupled wave calculations were performed. The rave equations wexe Fourier transfotned here, and a subset of the onedimensional k-space was numertcally solved in a periodic system of finite length. Figure $4-8$ shows that the linear instability theory 
indicatel by the solid line for these conditions to confirmed, if the $2 \mathrm{k}_{\mathrm{o}}$ dens1ty ripple $1 \mathrm{~s}$ gupressed. This calculation was conciucted at $\mathrm{n}_{\mathrm{o}}=.85 \mathrm{n}_{\mathrm{cr}},\left(\mathrm{v}_{\mathrm{os}} / \mathrm{v}_{\mathrm{th}}\right)^{2}=.1, \theta_{\mathrm{e}}=.75 \mathrm{KeV}, \theta_{\mathrm{e}} / \theta_{1}=3.33$, and $L=40 \lambda_{0}$. The standing wave was fixed at a given level, so that pump depletion was forbidden. The relative cuarseness of the calculational k-space was an inevitable consequence of the system discreteness. A longer system would resilt in finer resolution. The length used 1s, however, repreaentative of resent experiments. The one wave with $k<k_{0}$ which exhlbited exponential growth also had a real frequency to excellent agreement with linear theory. All the waves with $k<k_{0}$ were purely growlng, again in agreement with theory. If the background modiffcatlons were ignored, linear theory was well satisfied.

If the density was permitted to evolve without constratrits the spectra showed that the $2 k_{0}$ density ripple almost 1mediately iominated the ion waves. Unstable growth st1]l vccurred, but it was drastically modifled from that predicted by linear theory. The measured growth rates in the presence of the zero order density ripple but with a flxed amplitude purp are shown as $(\Delta)$ in Figure 4-8. The linearly fastest growing waves can be seen to be severely weakered, while previously stable waves with $k<k_{0}$ have been destabilized. Furthermoze, all the measured growth rates possessed elther no osciliatory component or one which was so small as to be unresolvable over $1000 \omega_{0} t$. In 
nether this case nor the above; though, did the fields coalesce inco self-consistent density wells.

Wave trapping was observed only in the coupled wave simulations when both pump depletion and the $2 \mathrm{k}$ o density fluctuations were allowed. In the above runs, the punp wave had been flxed. When it wis allowed to deplete, the spectrum sipread rapidly and simultaneous wave trapping/hole formation occurred. Figure 4-9 show a typical protile of the time averaged fleld and density, for $n_{0}=.95 \mathrm{n}_{\mathrm{cr}}$, $\left(v_{o s} / v_{t h}\right)^{2}=.125$, and $L=40 \lambda_{0} \cdot$ Comparison w1th other simulations at the same denstty clearly shows the disinctive density wells containing intense localized tield packets. The field magnitude ias been normalized to $\left(\mathrm{V}_{\text {os }} / \mathrm{V}_{\mathrm{th}}\right)$, so that the pump components initially had ampliture of unity, and the time averaged ptaks were at 2 . The single most intense peak in the Figure 4-9 has a magnitude $\left\langle A^{2}\right\rangle=25 A_{k}^{2}$, despite the presence of dissipation and punp depletion. The field spectrum in all trapping cases evolved relastvely early irto a coritinuous exponential shape as showp in Figure 4-10. This was woriclated with the field localization first, while the depth of the density hole increased continuously toward a teady value. This is consistent withobservatioss in RDF calculations that intense field localization would relax $1 \mathrm{f}$ the bole had not formed in approxinately an ion acoustlc time scale. As useful as these calculations were in interpreting the formative phase of wave trapping, hey colid not follow the evolution Into the stable vacuum hole state. As Figure 
4-9 shows, the hole collapsed indefinitely and when the reconstructed density becabe regative, the formulation became numerically unstable. Analysis of the fluid equatione used in the RTF calculations indirate that density defcmation may be pressure statilized. To be precise, sready driftless conditions require $d\left(v_{i}\right) / d t=0$. Neglecting, dissipative terms, this is possible when the plasma pressure gradients balance the gradients in the ponderonotive pressurs. Though formally the condition for steacy density is the vanishing of the civert ance of the Elux $n \bar{v}$, we expect the cancellation of these two largest force contributors to be roughly equivalent for practical purposes.

The steady density distribution car be derived fron the assumption of isothemaI pressure balance, regardless of the niechanisn responsible for the derortation. Pressure balence car be expressed mathematicaliy as

$$
0=-\left(m_{e} / R_{1}\right) v_{o s}^{2} \nabla A^{2} / 2-\left(\left(\theta_{e}+\theta_{j}\right) / M_{1}\right) \nabla n_{i} / n_{i}
$$

where the potential has been nomalized with respect to the vacuum: amplitude. This is easily solved for the physically reasonable boundary conditions

$$
A^{2}\left(x_{0}\right)=A_{\max }^{2}, n_{i}\left(x_{0}\right)=n_{\min }
$$

The solution in terus of the unspecified ronstants $n_{\text {nin }}$ and $A_{\text {max }}^{2}$ is 


$$
n_{1}(x)=n_{\min } \exp \left(-n_{0}^{2}\left(A^{2}-A_{\square i x}^{2}\right) / 2\right)
$$

where $\eta_{0}^{2}=\left(v_{o s}^{2} / v_{\rho}^{2}\right)$ and $v_{0}$ contains both electron and ion energy, $i \epsilon$. $v_{\theta}^{2}=\left(\theta_{e}+\theta_{i}\right) / m_{e}$. The amplitude, though nomalized to the vacuum pump strength, contalns any swelling iactors. : can further define $n_{\text {min }}$ in terms of $\Lambda_{\text {min }}^{2}$ and $A_{\text {max }}^{2}$ by noting that th density niaxina, which is approximately $2 n_{0}-n_{m 1 n}$, occurs at the ield minima. This redefinition simplifies the definttion in case of a standing wave, for which $\frac{A_{\mathrm{min}}^{2}}{2}=0$. The new definition then is

$$
n_{\min }=2 n_{0} /\left(1+\exp \left(n_{0}^{2}\left(A_{\max }^{2}-A_{\min }^{2}\right) / 2\right)\right)
$$

We can now insert the spatially modulated density function into the wave equation for the electromagnetic wave, and seek self-consistent solutions.

The electromagnetic wave equation has the new form.

$$
\frac{\partial^{2} A}{\partial t^{2}}-c^{2} \nabla^{2} A+\omega p^{2} \frac{n_{i}(x)}{n_{0}} A=0
$$

which is the equation derived by Kaw, et al. ${ }^{24}$. For small fieid strength, or at least small standing wave component, the exponential may be expanded to give a cubic nonlinearity. This nonlinear wave equation is commonly used in nonlinear optics. It has approximate solutions of the form $A \propto c n(x, m)$, where $c n$ is a jacobean elliptic 
function, $m=-\frac{a}{2 \sqrt{1-\frac{n_{0}}{n_{c r}}}}\left(\frac{V_{0}}{\gamma_{e}}\right)^{2}, \alpha=\frac{2{ }_{c r}^{n_{0}}}{1+\exp \left(-n_{0}^{2} A_{m a x}^{2} i 2\right)}$, for a standing wave, and boundary condfitions have been applied stich that $A( \pm \infty)+0$, as $\mathbb{t}+1$. The derivation is given in detail in Appendix $F$. The facobean solution results from a weak fleld approximaticn, yet we find that it possesses many of the same qualitative features as observed in simulations of wave trapping. It is therefore worth examining mare c]osely.

In the limit that the wave amplitude goes to zero, the cn-function. becomes simply the cosine function, with wavelength giver by the linear dielectric function. The wavelength, given by $4 \mathrm{~K}(\mathrm{~m})$ ( $\mathrm{K}$ a complete elliptic integral), and the peak amplitude, $c n(k, m)$ increase as the field strength increases, however. The m-parameter goverss the characteristics of each, and it possesses a complicated dependence cn density and field atrength. Figure 4-11 shows the vacum field strength dependence of the wavelength, for various densities between .25 and $.95 \mathrm{n}_{\mathrm{cr}}$. For moderate intersity, the fleld strength dependence is clearly weak below $.5 \mathrm{n}_{\mathrm{cr}}$, but pronounced above about $.75 \mathrm{n}_{\mathrm{cr}}$. As $m+1$ in fact, the wavelength goes to infinity and the functional form to a hyberbolic secant (sech). Although this approximate solution is expected tc breakiowr for strong fields, it exhibits the same. qualitative chaiacteristics as hole fcrmation. Because the onset of lccaliation, $i$ e. $\lambda+\infty$, is sudden and the structure which results 1s similar to the holes, we will define hole forming threshold as the 


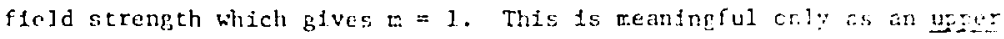
bound on the threshold, but it seill represents a corsiderahe improvement over previous trapplng criterla. Flgure $4-12$ shows the idpendeice of this threshold on density. Wote that the field strength, ( os it.' Is the value in the plasma not the vacuun one. These tho values are easily related by considering conservation of therg flux. Since $\left.v_{g r^{A}}\right|_{1}=\left.v_{g r^{2}}\right|_{2}$, we find $\left(v_{o s} / v_{t h}\right)^{2}=\left(v_{o s} j v_{t h}\right)^{2} v a c\left(1-n_{0} / n_{c r}\right)^{-i / 2}$. This is confirmed in RDF calcujations.

The above discussion concerned a linear swelling factor. As the wave pattern goes from a perlodic wave train to an isolated clectromagnetic pulse, the peak amplitude is expected to ce smplified. Te estlnate thls nonlinear steiling factor, note that the above rocicl is equivalent to $A^{2} /\left.\lambda\right|_{1}=\left.A^{2} i \lambda\right|_{2}$, for linear dispersion. It is not the purpose here to derlve a nonlineer dielectric function; but it is plauslble to extrapolate the sane fort to noninear condtions. This Is unproved, so its validity is judgeable by its success in predicting calculated field magnttudes. The nonlinear wavelength is given by $4 \mathrm{~K}(\mathrm{~m})$, a sample of which was displayed in Figure 4-11. Although this prescription is undefined at $\mathrm{m}=1$, the observed skelling factors are In reasonable agreement for fields such that $m$ is slightly less than untey.

Additional staulation characteristics can be uncerstocri as fell through this model. The observed hie width at half-deptin was Initially one-half vacuur wavelenrth, or $k_{j} j \mathrm{x}=3.14$. The width of the secant squared function at half-belght is $k_{0} \Delta x=2.7$. The difference 
Is of otier ten percent, whict: $:$ s about the uncertaincy in reading: icmputer generaced nlots. ilso, muletple holes kere soretimes

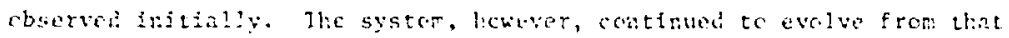
point, with the holes wentwa!ly coulescing*. This is certainly con-

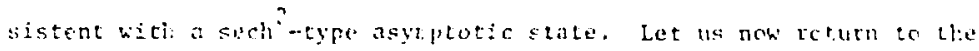
simalion results to seo if the relatively simple theory nids in cuartitativa understanding.

A series of pre calculaticns vere conducted at $r_{c}=.95 \mathrm{acr}$ in wich tho laser amplitude ras vaied to find the holo forming theshald in a finite sizec syster. In domping siss fucluced but for these rerposes there wist ne fon comperature. The borifortal axis in Figuro

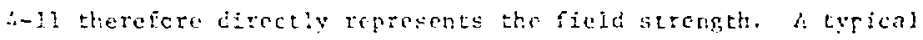

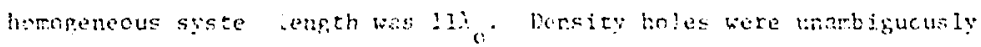
reserved for $\frac{2}{0} \geq 04$ unker these concitions. A field strength of $\eta_{c}^{2}=.5 n$ led to initial field localizaticr at the front edise of the piasm, but thes comvected ahiy as the front edge irefily expanded.

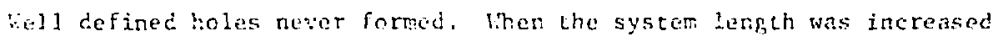
ic $14.3 \lambda_{0}$, however, all other conditions the same, a stable cavity of wictr. $51{ }_{c}$ and rinimur. density . n ${ }_{c r}$ formed at the erent edge. Clearly, a fleld strensti of $n_{0}^{2}=.50$ was in the transitional regime ire which leneth car play a role. at $r !^{2}=.36$ certain characteristics of the wave trapping frocess wer observed, but no holes ever developed.

\footnotetext{
${ }^{*} A$ discussion of the role of initial conditions on hole evolution is given in Section II.
} 


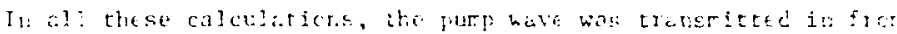

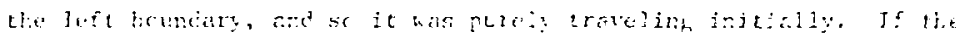

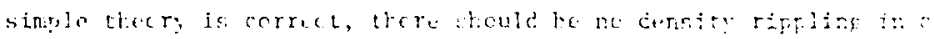

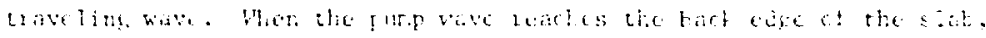

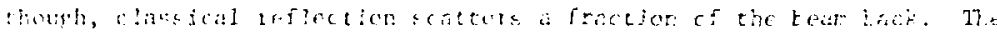

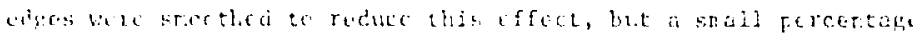

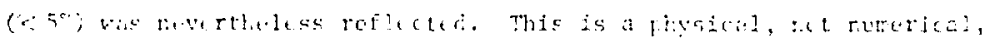

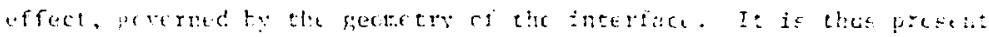

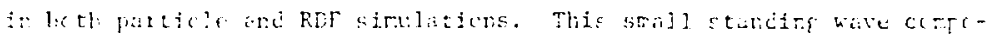

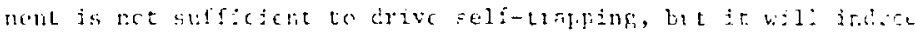

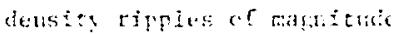

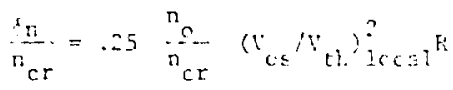

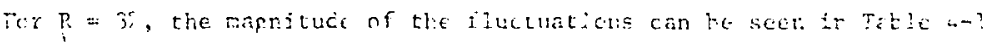

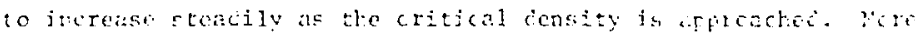

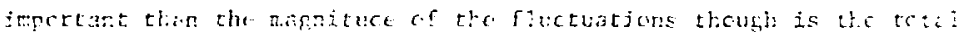

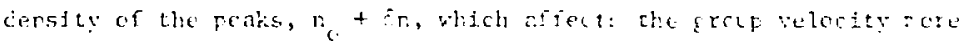

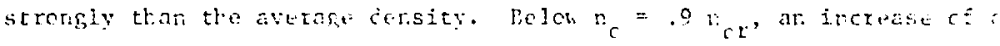

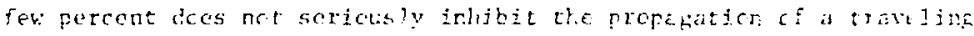

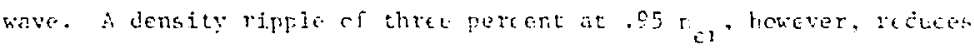

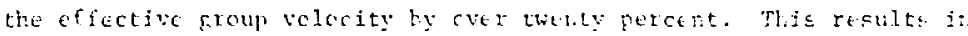
acciticral reflection, so thet further upstrear:* the stinding mave

\footnotetext{
We define unstream to be the directicli cpposite th that of the jaser, or rather points in space in tilat ditecticn. Dewestreari is hence in tie directien further alenf. tho lasel patl.
} 
Tabie 4-1. Fagnituce of Eensity rluctuations as a Function of Field Strongh ard Ambjent Cersits.

\begin{tabular}{|c|c|c|c|c|c|}
\hline 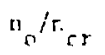 & .2 & .4 & .6 & .8 & 1.0 \\
\hline .75 & .0023 & .0045 & .0068 & .0090 & .0113 \\
\hline .80 & .0027 & .0054 & $.00 \varepsilon 1$ & .03 .07 & .0134 \\
\hline .85 & .0033 & .0066 & .0099 & .0132 & .0165 \\
\hline 90 & .0043 & .0085 & .0125 & .0171 & .0213 \\
\hline .95 & .0064 & .0127 & .C19i & .0255 & .0339 \\
\hline
\end{tabular}


component is tenhanced. In the fiedd of the streriser tatuding wuve component, deeper densily ripfles ore forned. One reeds orly a slight enhancement il: this marner to tave cue hurdrec fercent refaection upstrean. Once total reflection is achieved, Figure 4-1.1 was be used to estimatc the swellec field sirength and wavelengtr.

The total reflection results fron a boctstrup o.chenise. It depends on the amount of reflection at the back edge arre ch tho nagnitude of the denstly ripple which that reflection indices. The totai system lengch necded to establish totn? reflection thus increases as the pump strength accreases. At $n_{0}^{2}=.50$ the abuve analysis indjcales that a standing wave at full. plimp stronfth easily exceecs the fole formation threshold. The standing wave confonent hac ret reachec this threshold level by the Iront edge of sherter elat calculations. ihen the system length was ircreased by only therty-five fercent, though, the standing wave had been ijicreased to the poitit that hole threshold strength had been attannec. The sirulaticr at $r_{c}^{2}=.36$ inis not show self-trapping, but this intensity was apparently close to the threshold anyway. A much longer simulation might have resultec in trapping. The exact threshold for these unrealistic profiles, fonever, is secondary to the fact a rodel has been derived filch qualitatively and seni-quantitatively agrees with similations.

The analytic nociel for self-trapping, combined with the mechanics of standing wave formation, descrites the hole threshold near the critical denstty, but coes it also explain the simuition result; at lower density? In short, yes. The homogeneous simulations showed 
only SBS below about $.85 \mathrm{r}_{\mathrm{c} x}$, even when the vacultii stressth exceeded the andyzic threshold levels for hole fcmation. It the lower density, though, the starding wave could rot tootstrap efriciently. Consequently, only a strall fraction of the field was in a standing wave rore. The configuration of the simulatons favated the traveling wave rode; observation of waye-trapping resut tec onjy won a significant percentage of the wave could be conterted into stancing waves, i e. reer the critical density. The cnalytic rodel indinates that threshold for trapping loes decte. dramatically as the critical surface is approcched, tut the total nonchservation of wave trapping telow $.90 \mathrm{n} \mathrm{cr}$ was due to the traveling wave character of the laser. Understanding the phenonena better has therejore permitcec interptetation t.f what were otherwise baffling siculation results.

Before procedirg to the rore realistic inhomogeneous studies the resulas of this section should be sumarized. The key conclusion is that weve trapping, and hole fordiation, is a phenomena which nust be driven by a standing wave. Instable wave growth apgarently pIays a role during the formative phase, but this has not been identified with any linear instability. Such a linear perametric instability was discovered, but the self-consistert growth of the waves in the presence of a lineariy driven density ripple drastically modiefed the growth spectrum. The free response of the plasma to the electromagnetic field of sufficient intensity was required to observe the localized state. The field spectrum of this loculized state was - early exponential, leading to the supposition that the statle state was akin to a 
solltary wave. Such solitary waves may be regarled as nenlinear nomal modes of the [1]asna, in the presence of a standing wave. This is consistent with the steady-state analysis of the field-ciensity distribution. The field distribution which was reconetructed from a weak-field ipproximation was found to possess the trafped stata characterlstics seen in the simulations. Tinally, synthesis of these results led to the conclustion that the absence of holes below. $90 \mathrm{n}$ cr was due to 1nablity of the homogeneous slab to establish a standing have of suffictent aagnitude. Numerical threshold values were calculated analytically, but it is doubtful thet the homgeneous threshold is of much practical value. If holes are co be signif.cant in laser fusion it is necessary that their threshold in inhomogeneous plasmas be known. Nevertheless, the cubic nonifnearity model can be expected to provide an upper bound for t.ungeneous systems. 
Wave Trappingificle formation ir. Irhomogencous Plastar

Whenever a densiey profile extencis far above critadid denstey, some iraction of the energy will gererally te reflecter, biving a standirg wave comporent, ihile the standing have comorent rny lie at a significant levei in the iahorogennous plasma, the inhomogeneites thenselves may spoil the trapping. Numericas studies here therefore perfermed to understand inhorogereity effects or vave trapplng.

The homogeneous model for stif-trapping vas based on an exprnential ronlinearity in the field, abbejt truncated to first-cruer tems. This rodel can be easily extended to a linear density frofile plasma. The wave equation in a lineas profilt is the Airy equation discussed in Chapter 2. Larlfer in this chapter, we derived an expression for the steady censity distribution as a function of intensity, Fo $(L-3)$. Tf the ambient density in that expression is replaced wth a linear gradient density function, $n_{c}(x)=\left(1-\frac{x}{L}\right)_{n_{c r}}$, a type of nonlinear Airy equation is produced

$$
\frac{\partial^{2} A}{\partial x^{2}}+\frac{a_{o}^{2}}{c^{2}}\left(1-\frac{2 n_{c r}\left(1-\frac{x}{L}\right)}{\left(1+e^{-1 / 2} n_{0}^{2} \Lambda_{\max }^{2}\right)}\right) e^{-1 / 2 n_{c}^{2}\left(A^{2}-\Lambda_{\max }^{2}{ }^{2}\right.} A=0
$$

Analytic soiutions of this equation are unknown, so it was solved nuticically. The solution characteristics were found to be similar to those of the jacobian function. As the pump power increased, the field experienced both a swelling in amplitude and a dilation in wavelength. 
This letter strengly affected the inhomcgeneous solutien, since the swiling cepends on the number of wavelengths which fit or the profile. Ir conseguence, the norlinear swellirg of the fielc was atcenuated by ste rifertive reduction in the profile length. This simple frted, malvely inserireted, loes not represent the pisysics accurately at all, Since the nonlinenr evolution of the itrear profile is initjatec by an Aly-like intensity patern, ore would expoct the strongest and Carliest efferts to occur near the critical density. Orce the fierturbations reach signjficant levels, ficwever, they wijl tend to lock the adtoional swelding in thia field into the denstey rroughs. The artual norlinear state is thea ceterrined by the competition tetween the terdency of the field to increase in weveiength and inerta of the perturbed plasma which remembers its initial spatial distribution and atcerpts to resst walcheth dilation. These intuilive ideas were lised to refine the density nodulation model in $\mathrm{Eq}(4-7)$. The exporiential ronlinearfty was retained, but only the linear Airy function appeared in the nonlinearity. Despite the possibility that this could underestimate the nonlinear field effects, the computed intensity partern exhbited huge swelling factors in modest profile lengths at moderate field strengtirs. Figure 4-13, for instance, shows the mudel results for an $l=10 \lambda_{0}$ density profile when $\left(v_{o s} / v_{\theta}\right)^{2}=.3$. The linear swelling anounts to a factor of 3.6 ( $x$ 4 for comparisor with traveling waves) for such a scale length. The inclusion of density rodulations has amplified the peak intensity to cver 18 . The minirum density in such a field moreover is down to. $1 \mathrm{n}_{\mathrm{cr}}$ as show in 
Figure 4-14. The model calculatinn Lius show a hole forming threshold for this scale length of $\left(v_{o s} / v_{\theta}\right)^{2}=. ?-.3$.

The IInear swelling, In the Intensity pattern Increases with scale length. Since this model uses the Iinear pattem to evaluate the density perturbations, the hole threshold should decrease with increasing scaie length. Tie defintticn of threshold for a crntinucus process is somewhat arbitrary, but a plousible one 1s: that vacum field strength for which the minimum density first falls below $.01 \mathrm{n}_{\mathrm{cz}}$, Thon the model calculations confirm the decrease in thrashold. Figure 4-15 gives the calculated tole threshold as a function of linear scale length.

Particle aimulations were also perforne? to study self-trapping in inhomegeneous plasmas. These yielded consistently higher thresholds than did the model calculations discussed above. If allowance is made for the energy SBS is removing from the beam under these conditions, however, this result is not surprising. The rule of thumb for SBS Is that fif fy percent backscatter occurs whenever $\left(v_{o s} / v_{t h}\right)^{2} \frac{\lambda_{0}}{L_{0}}=5-10$, dependirg on the fon damping rates. As an example of the effect of such scattering, consider a simulation conducted on an 8 wavelength Iinear density profile, with electron to fon temperature ratio of 10 . Ion landau dampting is thus weak, and the lower linft on the SBS prescript on is probably applicable. One should thus expect strong SBS above $\left(v_{o s} / v_{t h}\right)^{2} \approx .6$. The adjusted field strength threshold for holes on this profile is $\left(v_{o s} / v_{t h} j^{2}=-5\right.$ 
$\left(\left(v_{n s} f v_{e}\right)^{2}=.45\right)$. Holes ded rot form, thowever, in a simulation for which $\left(V_{o s} / v_{t h}\right)^{2}=75$. Thaminimum density near the critical density renched onlv $3 \pi_{\mathrm{cr}}$. This minimum teat the critical density is consistent with the model calculations, if the energy reaching tine critical density were reduced by fifty to sixty perceni. Under the same conditions, a fleld strength of 1.1 resulted in stable varutur holes, as Figure 4-16 Indicates. Assuming that particle trapping limits the average scatteling factor, to say fifty to seventy percent, this calculation is also roughly consistent with the mogel derlved threshoids. RDF simulations in profiles up to 20 wavelengths lang behave in a similar fashion.

The auove examples indicate that beam modification in the underdense plasma rust be included in evaluating self-tzapping thresholds in ichomogeneous plasmas. The only stimulated effect aliowed in these one dimensional stmulations was Brillouin backscatter, and knowledge of its nonlinear behavior permfted fairly accurate estimation of its effects. Since SBS has inhomogenecus threshclds close to the wave trapping ones, it mist probably be considered whenever the latter threshold is being evaluated. There are other effects, however, which are also efficient at reduclig the energy flux to the critical density. Since wavetrapplng has its lowest threshold in that region, the local density near the critical surface is the parameter which deteruines whether holes will form or not. Raman back- and sidescatter and Brillouin stdescatter are exanples of flux reducing inechanjsms which can not be readily addressed in this study, but which pose considerable 
problems to hole formation. Likenise, any strong disipation mechanism would absorb the energy and hence Inhibit hole formatioi:. Although $1 \tau$ is not practical. to categorically 1 ist all the effects whtch could influence wave trapping, any effort aimed at providing more realistic threshold conditions must consider some. Brillouin scattering has already been discussed. As further iliistrations, classical absorption and finite bandwidth will be briefly discussed below.

As the 1lnear swelling studies in Chapter 2 showed, the primary effect collistonal damping exerts on the intensity pattern is a uniform decrease in its magnitude. Calculations over a wide range of scale lengths indicate that the curve in Figure $(2-5)$ is useful for estimating this magnitude, within roughly ten percent, as a function of total absorption. For moderately strom absorption the decrease in $\left\langle A^{2} ;\right.$ mean squared amplitude, can be nearly a factor of 2 . This reduced effective intensity is then the parameter to be used in evaluating hole thresholds.

Finite bandwidth effects on the intensity pattern were also studied in Chapter 2. For realistic bandwidths, the incoherence related reductions tended to be small, 1 . e. reductions of twenty to forty percent for scale lengths between $20-100 \lambda_{0}$. This factor must be cautfousiy interpreted. Tespite relatively minor attenuation in the intensity pattern, bancwidth also severely weakens rcsonant parametric instabilities, such as SBS. Inhibition of scattering Instabilftes in the underdense plasma will allow a greater fraction of the originai 
beam to reach the critical surface. The net result of these two opposing tendencies on wave trapping is not generally clear. To help resolve uncertainties, severai simulations were conducted in an S wavelength long density profile, with varying amour:s of b.andwidth. The incident field strength was $\left(V_{o s} / V_{t h}\right)^{2}=1.1$, sufficient to create vacuum hole: after $\omega_{0}^{1}=500-800$ with a nonochromatic wave, when $\frac{m_{e}}{M_{i}}=.01$. The results for bandwidths of $\Delta \omega / \omega_{0} \neq .03, .05$, or .10 were that virtually no djfference was observed in either hole formation or subsequent evolutiun. In fact, if there was any difference in the calculations it was that the hole seemed to form slightly earliar in the large bandwidth cases. These calculations do not form a definitive study but they Indicate that the net effect of bandridth under nonlinear conditions may be to increase the energy which actually reactes the critical surface.

It is of considerable interest to study the competition of the anomalous abscrption mechanisms near the critical surface with wave trapping. Development of strong holes will conceivably disrupt the critical surface to the extent that collective processes will be gradient stabilized. On the other band, if microturbulence is established first, the enhanced dissipation could lower the local field level below hole-forming threshold. To further complicate matters, resonance absorption may find the "Uumpy" surface a hospitable envizonment, while the otfar processes are spoiled. Unfortunately, only the self-consistent two-dimensionel study of these interacting 
processes will be able to provile answers tc these questions. The one dimensional calculations employed herein are not adequate for this problem.

Inhomogeneous thresholds have been studied in this section, and several useful guides have been derived. Nodel calculations were used to find thresholds as a function of density scale length. This threshold decreased with fncreasing scale length, due to the length dependence of the linear swelling factor. The model calculations relied on this swelling factor, which may have tended to cverestimate the thresholds. As an upper bound, though, the calcuiations, as represented in Figure 4-15, were consistent with simulations. Incertaintles were introduced when stimulated brillouin scattering was allowed in the calculations, for they reduced the energy fluy to the critical surface. Brillouin scattering covld be estimated from cther nonlinear studies, and allowance for stimulated reflection brought: simulations into closer agreement with the model predictions. SBS is representative of a body of phenomena which can occur in the underdense plasma, but which do not effect the critical surface reginn irectly. This led to the general rule, that or.ly the local energy density near the critical density was needed to evaluate hole thresholds. The question of competition between holes and anomalous abscrption In the same region, unfortunately, must await higher dimensional simulations to be resolved. It is finally appropriate to discuss the:e studies within the overall efforts which have been made to understanc wave trapping. 


\section{SECTION III}

Discussion of Wave Trapping/Hole Formation Results

Self-focusing and self-trapping of 1 thense laser beams in lispersive media were first investigated whthin the context of nonlinear optics (c.f. Ref 16). The saturable cubic nonlineartty used in those studies is also useful in plasmas, but the saturation phystcs is quite different than in dispersive 11quids or solids. The nonlinear optics work thus dices not possess direct applicabillty to laser fusion plasnas, and will hence not be considered further. Perhaps the first investlgation of condensed electromagnetic statrs in plasmas was conducted by volkov ${ }^{31}$. Surovich and Karpman ${ }^{27}$. however, were the first to phrase the problem of trapped electronagnetic energy in density wells properly. They discovered a number of solitary wave states depending on the ratio of the electrmagnetic to fon acoustic group velocities, and on whether the dielectric function was positive or negative. In particular, when the former ratio was Iess than unity, they found a traveling wave broke up into a solitary save with the characteristics found for holes, excent that the coupled density/field structure propagated at the field group velocity. For E $\cdot 0$, their analysis uncovered stationary solitons, similar to the one found in Section $I$. In the negative-E case, however, they linited themselves to small amplitude pumps. In subsequent papirs they studied the stability and effect of dissipation on this negative-E soliton. 
The analysis of negative- electromagnetic wave states tals teon futher treated by Narburger and Tooper ${ }^{32}$, who recognized the standing rave nature of such a sclution, but kept the ions tritotile, and by Lai ${ }^{33}$ who relaxed the ion inmobllity assumption and found one dimensfonal, hole-like solutions in the overdense plastra.

Kaw, Schmidt, and wilcox ${ }^{24}$ performed an elegant analys fs of the exponentlal saturating nonlinearity used in $\mathrm{Eq}$ (4-5). They derived both filarentation, or self-focusing, and self-trapping from this equation. The filamentation admitted of a legitimate stability analysis. Their analysis also rointed to an asymptotic waveguidelike solutions. Although they did not explicitly state the jmportance of standirg waves for the self-trapped state, their form for the exponential nonlinearity impled it. A stability analysis for such condictons was inappropriate, as demonstrated in Section I, but they did find eigerstates which possessed cavity-like properties. Threshold for this eigenstate was vague but the explicit exanples given were for $\left(v_{o s} / v_{e}\right)^{2} \sim 1$ and $\varepsilon<0$.

The condition of negative- $\varepsilon$ for self-trapped states has been generally translated into occurrence only in overdense plasmas. The homogeneous calculations in Section I show that this is overly restrictive, if not actually misleading. Localized wave states can easily evolve from profiles which are initially everywhere underdense. Even the suggestion by kaw, et al. that growing ion waves could trap the field did not differentiate between normal fon acoustic nodes, which did not trap the field, and zero frequency censity fluctuations driven by 
standing waves. Th:is is significant primarily in that it shows that the localized state is rot the usual erci product of SBS.

The soliton state which Gurovich and kirpmar ${ }^{27}$ derived for positive-e is limited to density close enough to the critical density that the ion acoustic speed is sreater than the field group velocity. This occurs only when $1-\frac{\ell}{m_{e} c^{2}} \frac{m_{e}}{M_{1}} \leq \frac{n_{0}}{n_{c r}} \leq 1$, which occupies an axcerdingly small vclume. Although this state could evolve fron: SRS, it $j$ s much less likely than the one eriver by a standing wave.

$\Lambda$ growing experimental anc theoretical literature is occurrine about localized electrostatic cavities, or "cavitors", rear the critical density. Since the forn of the coupled density/electrostatic field equations is simjar to that for electromagnetic waves, this is hardly surprising. If the electrotagnetic fieli localizes as a soliton, then the cavitons may be regarded as Langmutr soiitons. It is not necessary here to discuss the theoretical developnents in detail; it should be noted that the studies of Zaliharov ${ }^{34}$, valec and Kruer $^{35}$, Schmidt ${ }^{36}$, and Denavit, Pereira, and Sudan ${ }^{37}$ tend to cverlap with these wave trapping studies, except that the former adnits the possibility of direct wave-particle interactions.

The close relation between the two phenoriena is illustrated by the two and three dinenstonal studies of Denavit, Perelra, and Sudan 37 , and those of Valeo and Estahrock ${ }^{29}$. In the former case, the initially slab electrostatic state was found te be unstable tc sausege-type instabilities. The final state was fcund to be locilized, electrostatic "bubbles". Vajeo and Estabrcok founc alrost precisely the sance 
behaviot for the electromagnetic waves. The latter, however, exhibited no tenciency to dissipate late in time. Both anaiyses were confirmed with computer simulations. Langdon and Lasinski ${ }^{30}$ have also confimed the two dimensional behavior of the electromagnetic state, and have uncovered interesting characteristics of the trapped fleld.

Experimental studies of cavitons have been conducted with microvaves by Kim, Stenzel, and Wong ${ }^{38}$. Experiments in laser plasmas by Fidman and Sigel ${ }^{39}$ for Nd lasers and Donaldson and Spalding ${ }^{40}$ for $\mathrm{CO}_{2}$ lasere have reported curious structure near the critical surface. The $\mathrm{CO}_{2}$ experiments actually mapped an extensive cavity in the density profile, but the dimensions, $50 \mu \mathrm{m}$ by $200 \mu \mathrm{m}$, are much larger than any cavity seer: in simulations to date. This, however, may be due to the limited space or time covered in the simulations. Other experiments have tentatively cbserved extraordinary roughness of tie critical surface, which could be a signature of electromagnetic "tubbles". None of the experiments reported to date, though, can claim unambiguous detection of the lacalized state.

The conclusions fron! this study may be briefly sumarized. One dimensicnol thresholds for wave trapping/hole fornation near the critical density were found tc be competitive with those of strong Brillouin backscatter. Although extensive study of tine competition of this effect with other collective processes was not feasible within the cne dimensional framework, those effects may be estinated roughly by evaluating the extent by which they reduce the energy density in the region of tha critical surface. Finte bindwidth in particular 
seemed more effective in spoiling resanant collective processes, j. e. SEs, tian in inhibiting hole formation. 


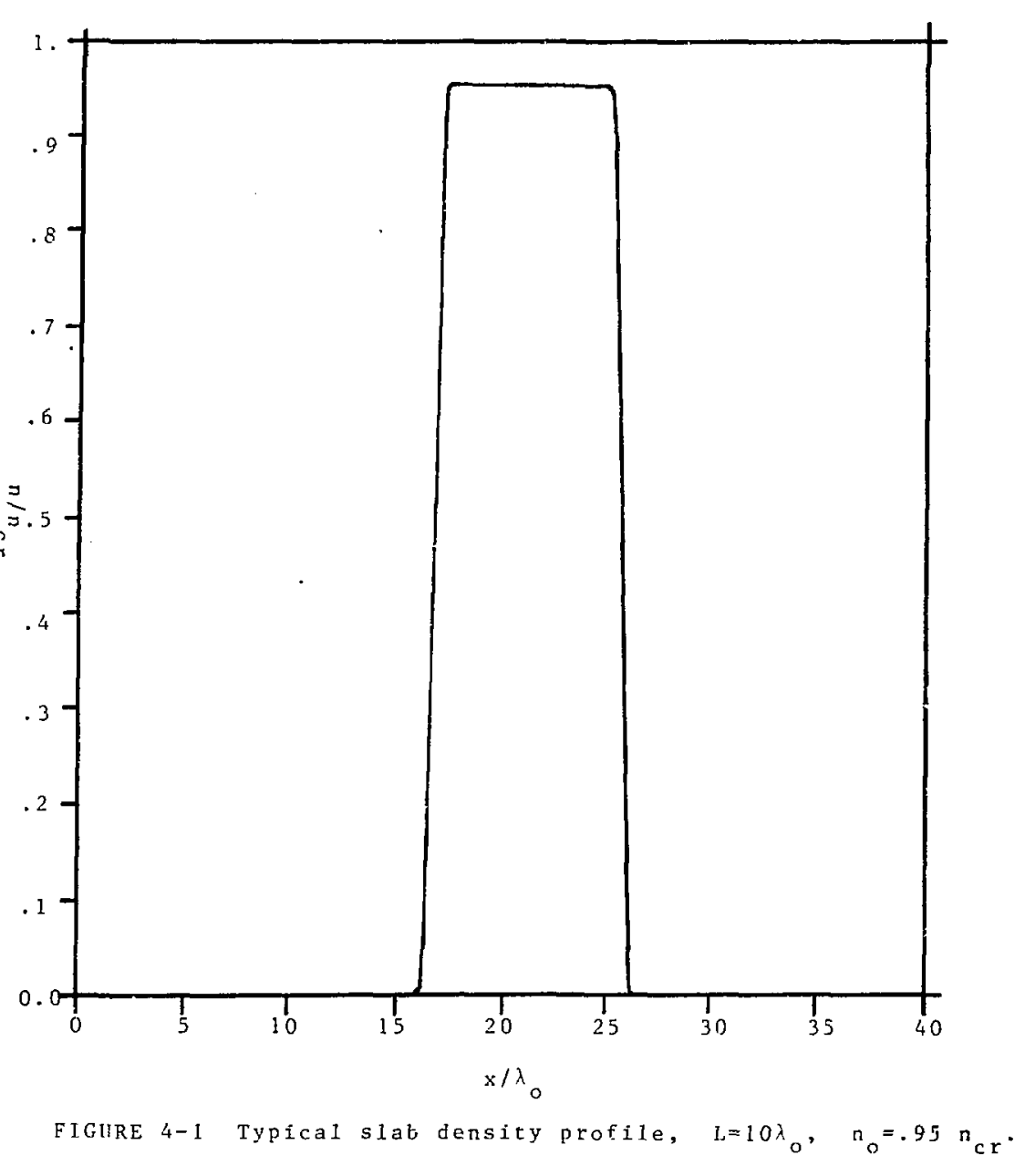




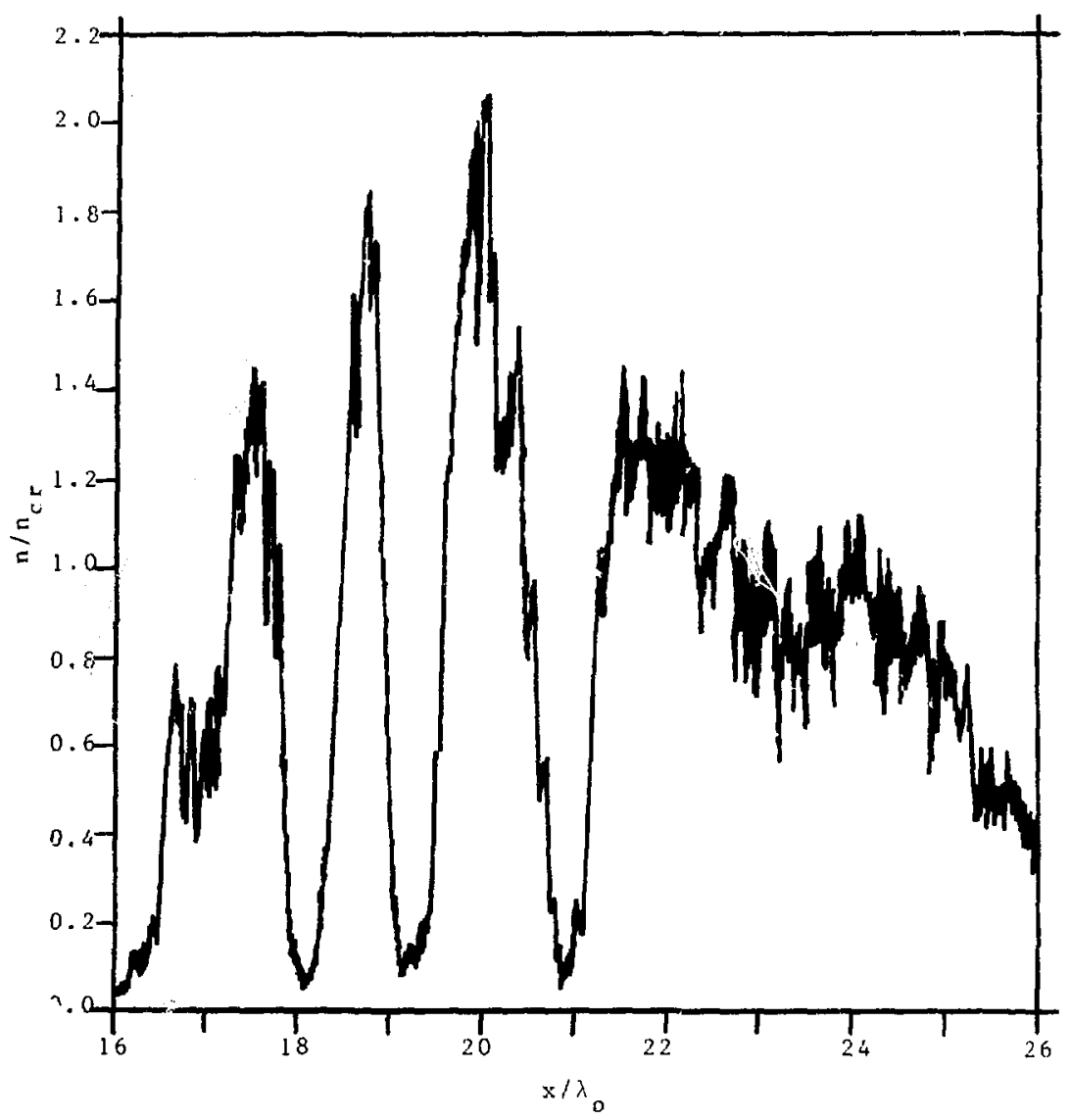

FIGURE 4-2 Densicy profile atwo $=500$; initial profile length, $L=10 \lambda, \quad n=.95 \mathrm{n}$, Well defined holes are cencered at $\times 9 i_{0}=18.0,19.2,20.8$. 


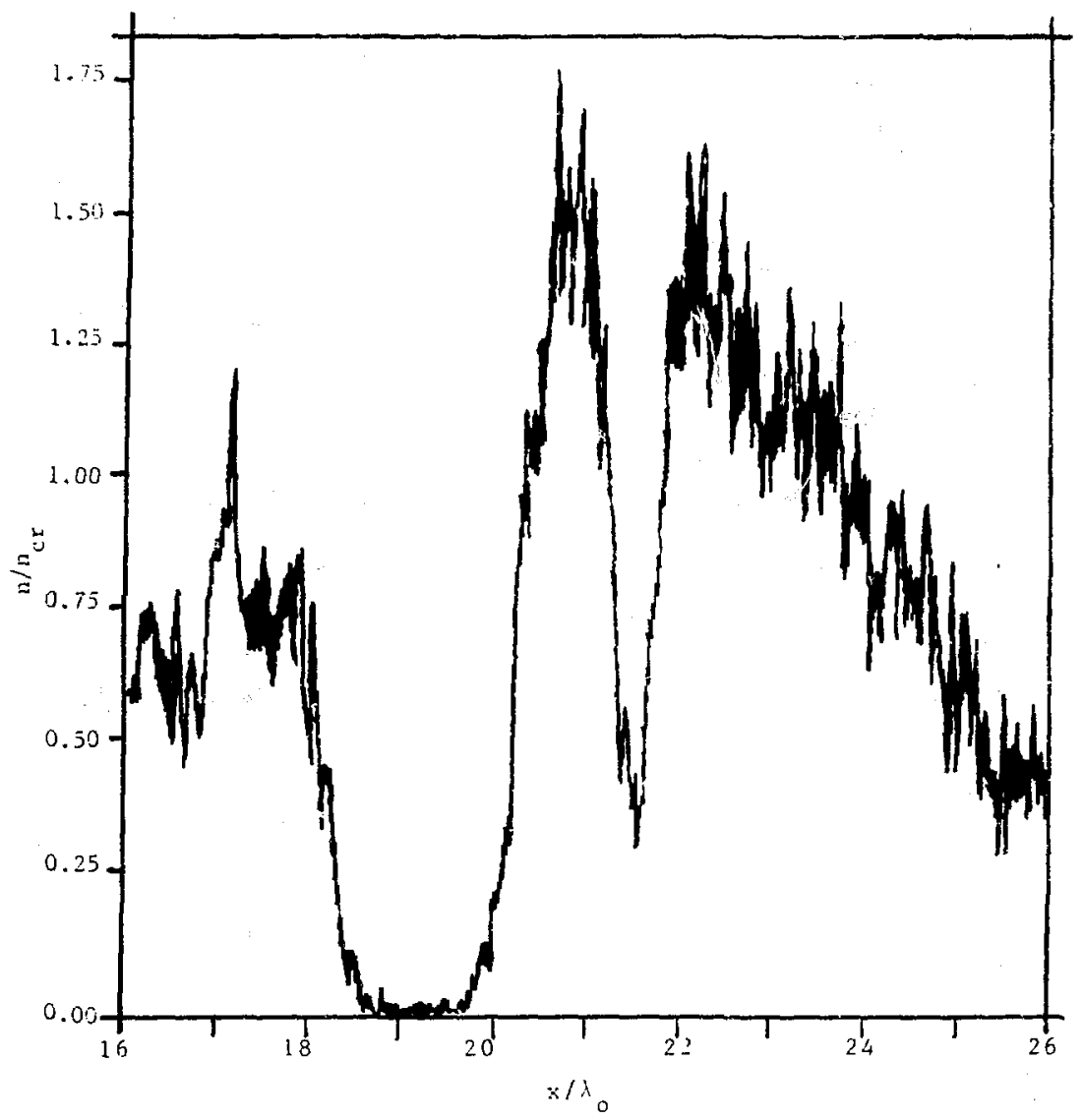

FiguiE 4-3 Density profile at $\omega_{0} t=2000$, same calculation as shown in Figure 4-2. Hole $a t^{\circ} x_{i} \lambda^{2}=19.2$. Nate under-

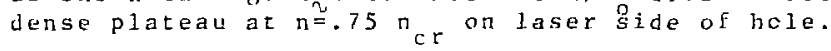



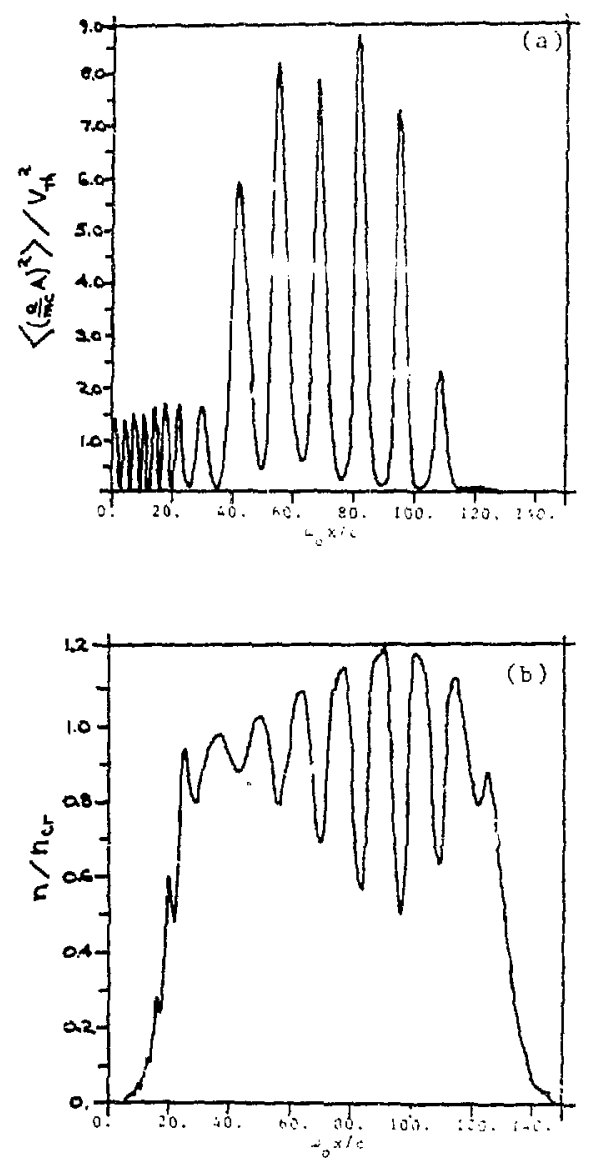

Figure 4-4 (a)Time-averaged field prosile at w $\mathbf{r}=1200$. Peaks are swelled by a factor of 2

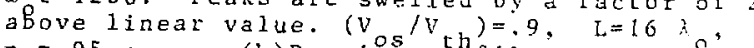
$n_{0}=.95 n_{c r}$ (b)Densiey profile at same Eime as (a). cr The beginnings of fiejd codlescence can be seen at $\omega_{0} x / c=56,70,82,96,109$. 

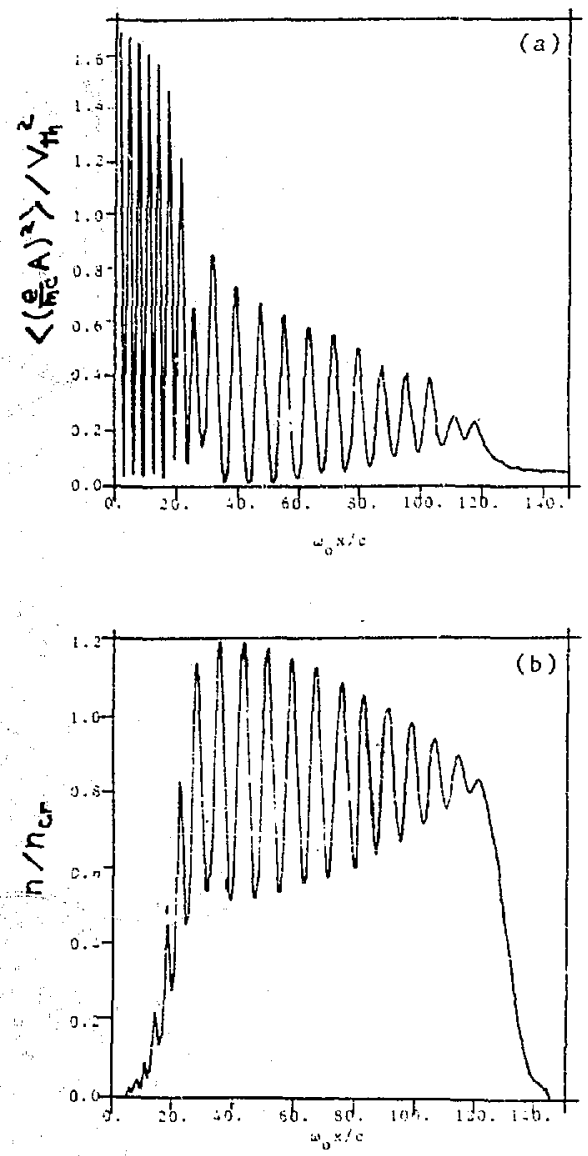

Figure 4-5 (a)Time-averaged Eield protile at $\therefore t=1200$. An exponentially back-amplified signal is evident, though peaks are smaller than linearly swelled standing iave. $\left(v_{o s} / v / h\right)=.9$, $\mathrm{L}=16 \mathrm{~A}^{\mathrm{r}} \mathrm{n}_{\mathrm{o}}=.85 \mathrm{n}_{\mathrm{cr}}$ (b) Density profile at the 

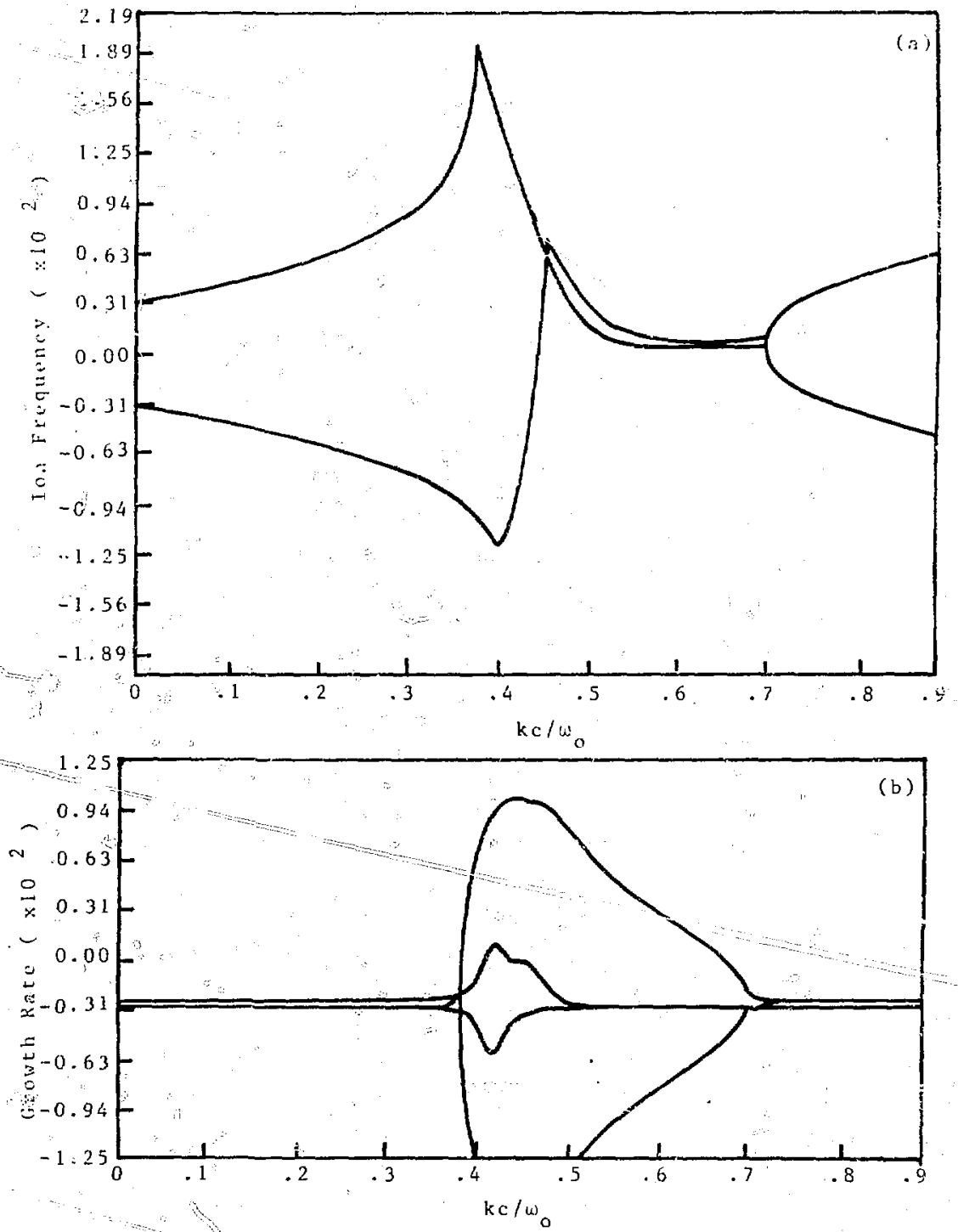

Figure 4-6 Numerical solution to dispersion relation for parametric coupling of electronagnatec waves and ion density fluctuations,

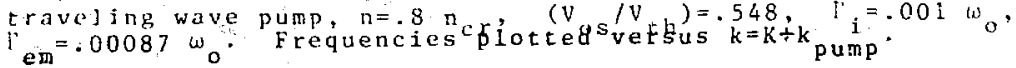



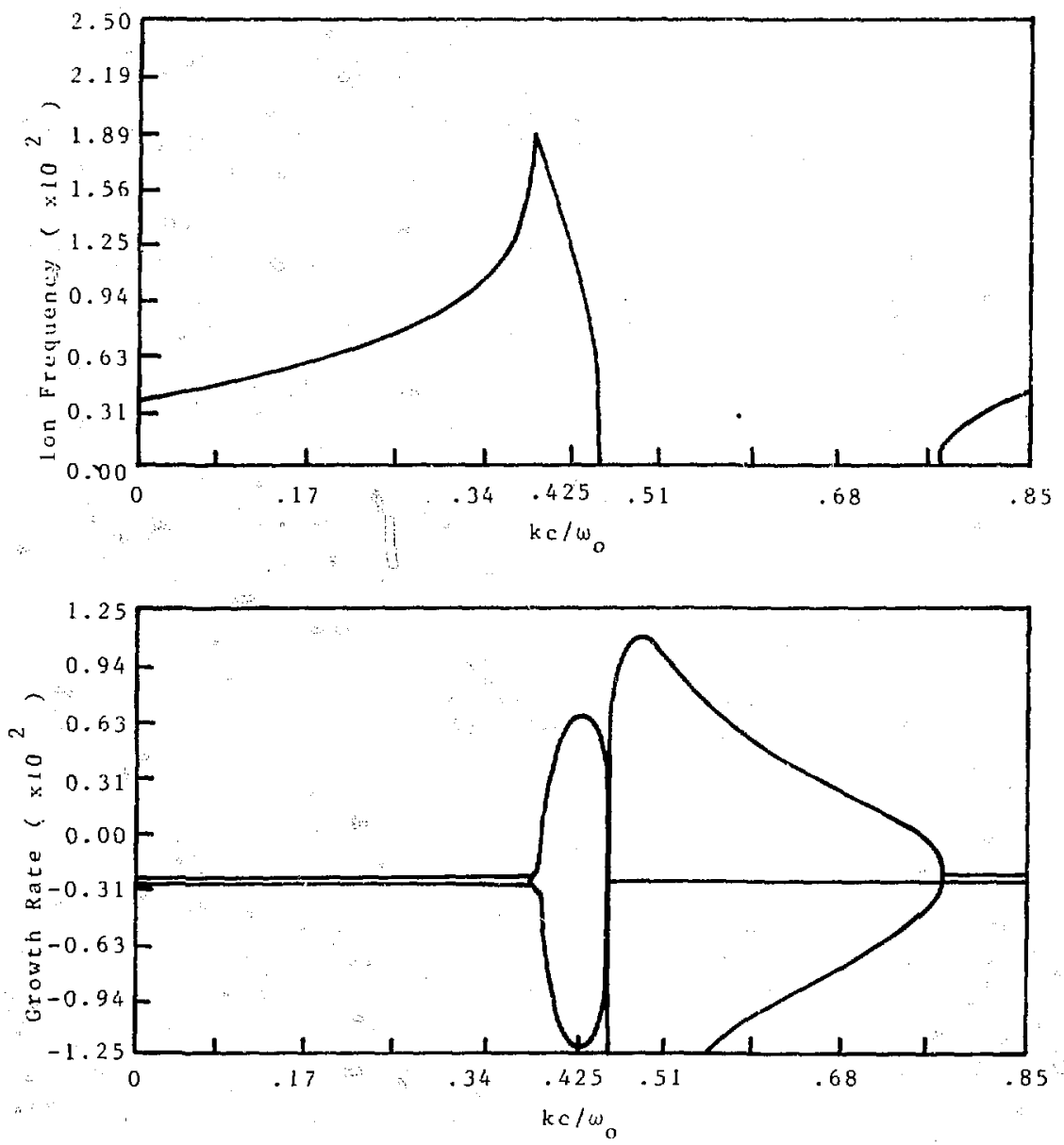

Figuie 4-7 Numerical solution to dispersion relation for parametric coupling of electromagretic waves to ion density fluctuations, standing wave putlp, $n=.8 \mathrm{ner}\left(\mathrm{V}_{\mathrm{OS}} / \mathrm{V}_{\mathrm{th}}\right)=.447, \Gamma_{i}=.001 \omega_{0}$, $r_{e m}=.00087 \mathrm{w}_{0}$. Wlotting convention the same th in Figureit-6. 


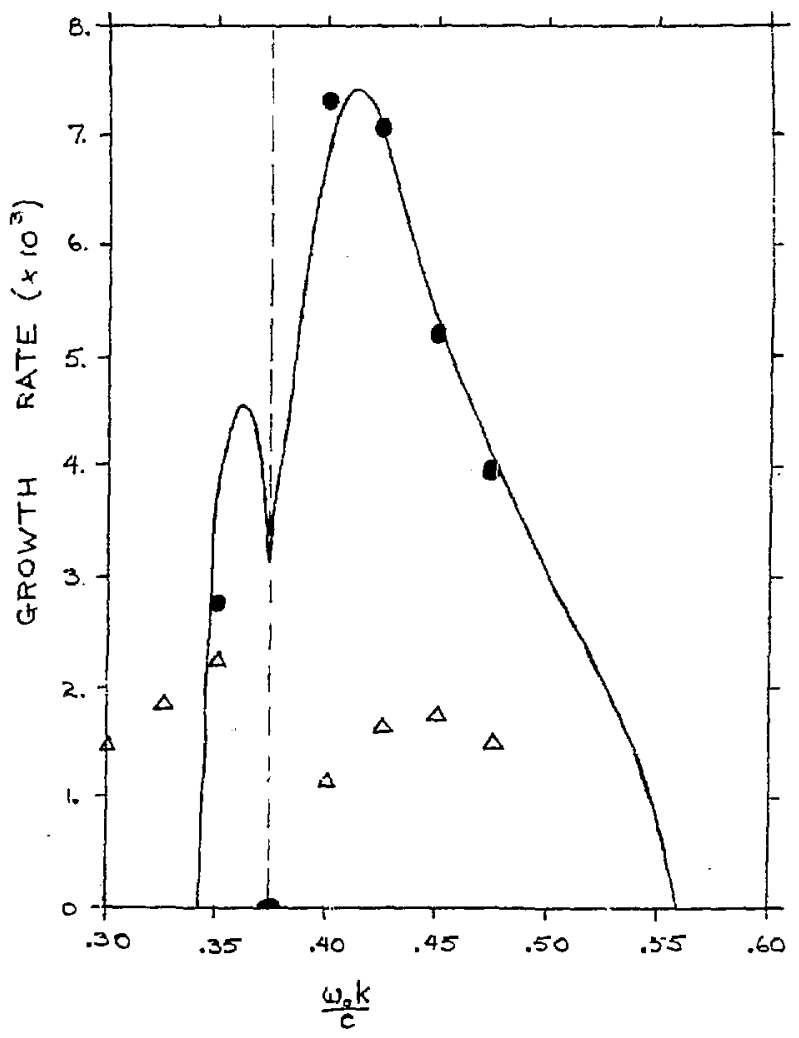

Figure 4-8 Growth rates measured in a coupled wave simulation, standing wave pump, $\left(\mathrm{V}_{\mathrm{os}} / \mathrm{V}_{\mathrm{th}}\right)=.316$, $\mathrm{n}=.85 \mathrm{n} \mathrm{c}^{*} \Gamma_{j}=.0025 \omega_{0}, \Gamma_{\mathrm{em}}=.001 \mathrm{gs}^{\mathrm{s}}$. th solid line is theoretical prediction for these conditions; solid circles( ) are measured growth rates when $2 k$ density ripple is artificially suppressed; triangles $(\Delta)$ show growth rate when density ripple allowed to grow. 


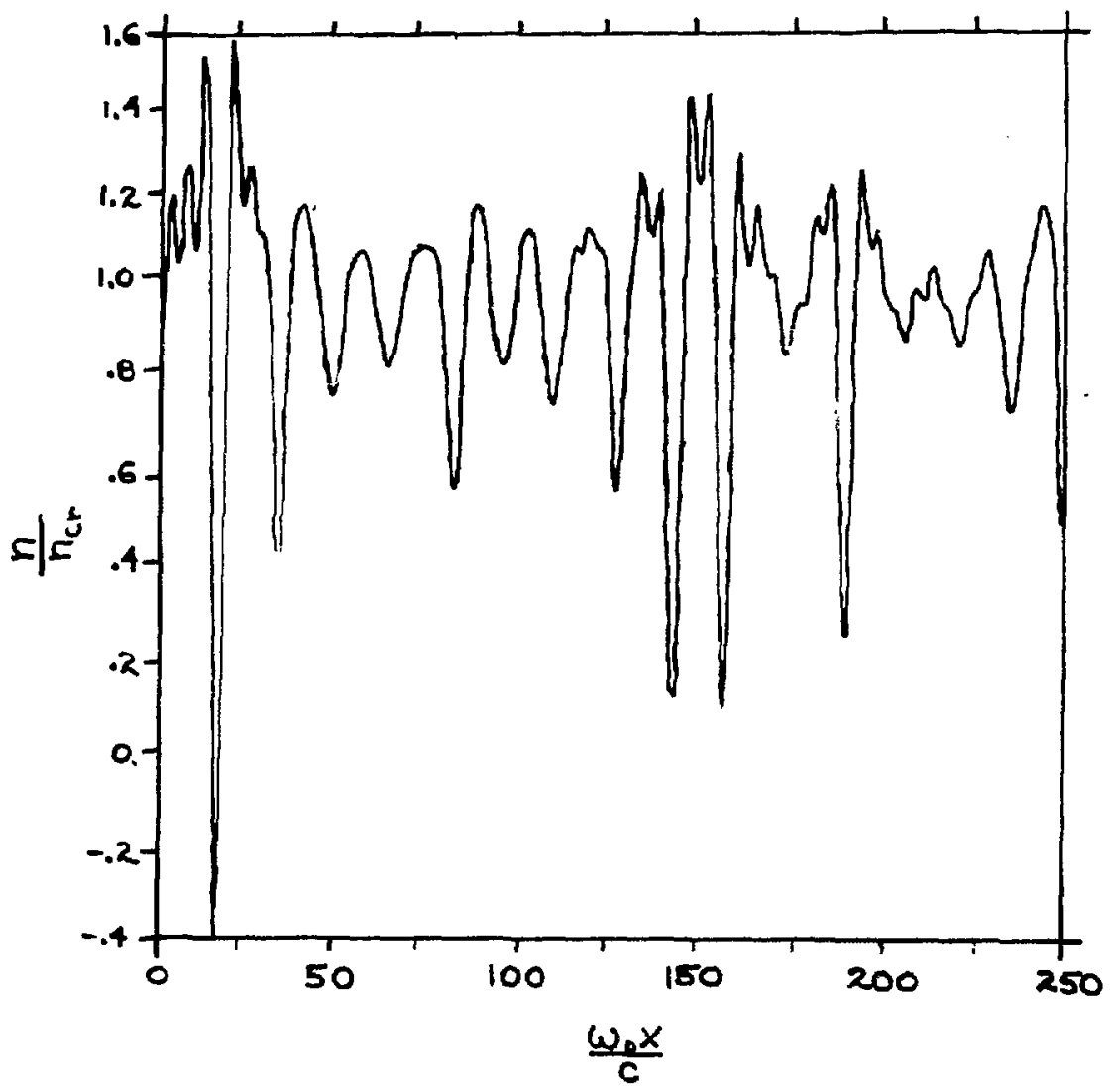

FIGURE 4-9(a) Reconstruzted density profile from a coupled wave simulation. ( $\mathrm{V} / \mathrm{V})^{2}=.125, \mathrm{~m} / \mathrm{M}=.01, \mathrm{n}=.95 \mathrm{n}$ cr.
$\mathrm{L}=40 \mathrm{~A}^{\circ}$. Note negative density trough at $\omega \mathrm{x} / \mathrm{c}=28$. Fourier codes do not prohibit such benavior but become rumerically unstable when it occurs. 


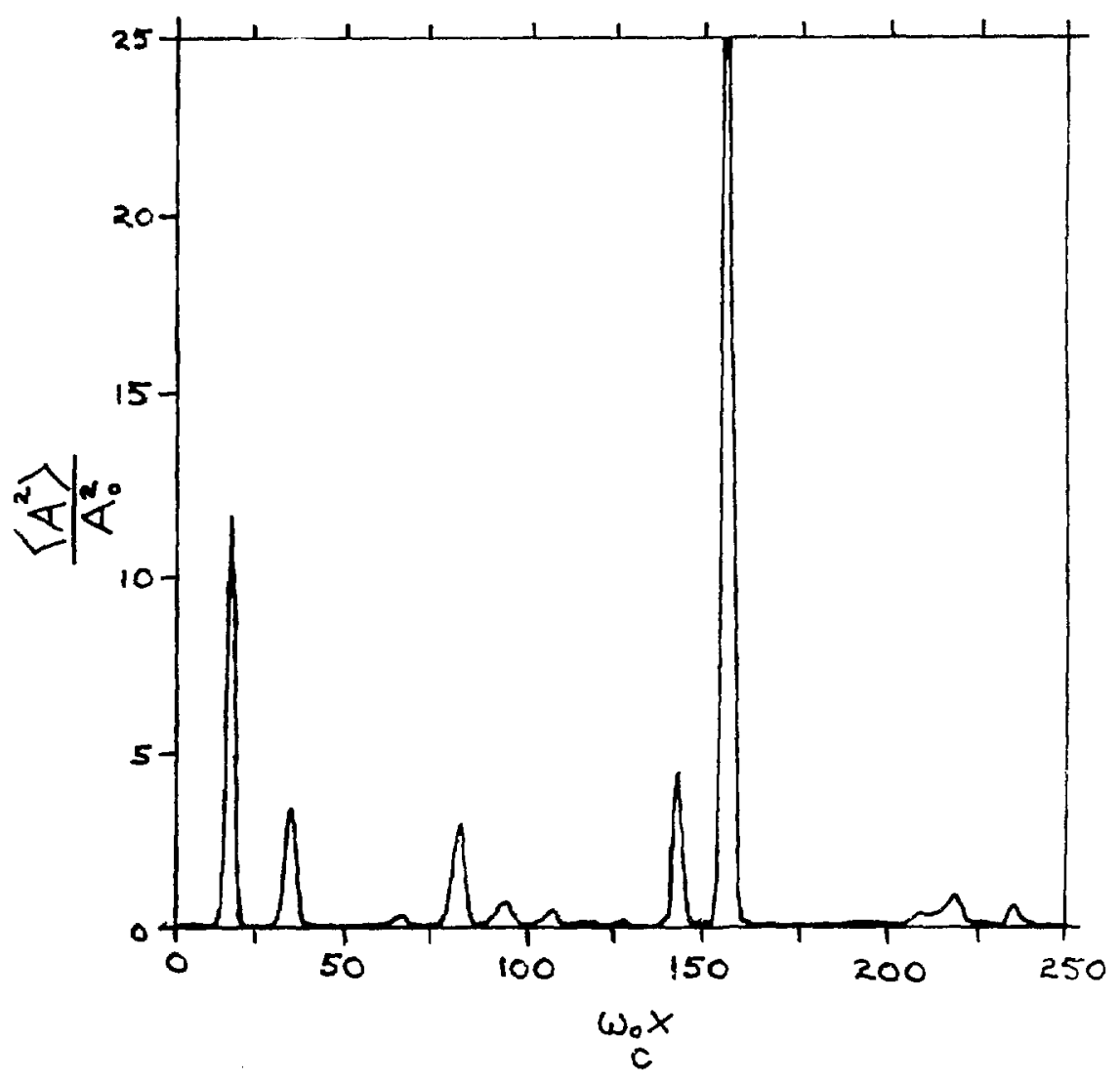

FIGURE 4-9(b) Reconstructed intensity profile at sing : :me as (a). Peak at $\omega_{0} x / c=160$ is $A^{2}=25, \ldots, y_{1} \mathrm{red}^{2}$ with $A=\ldots$ 


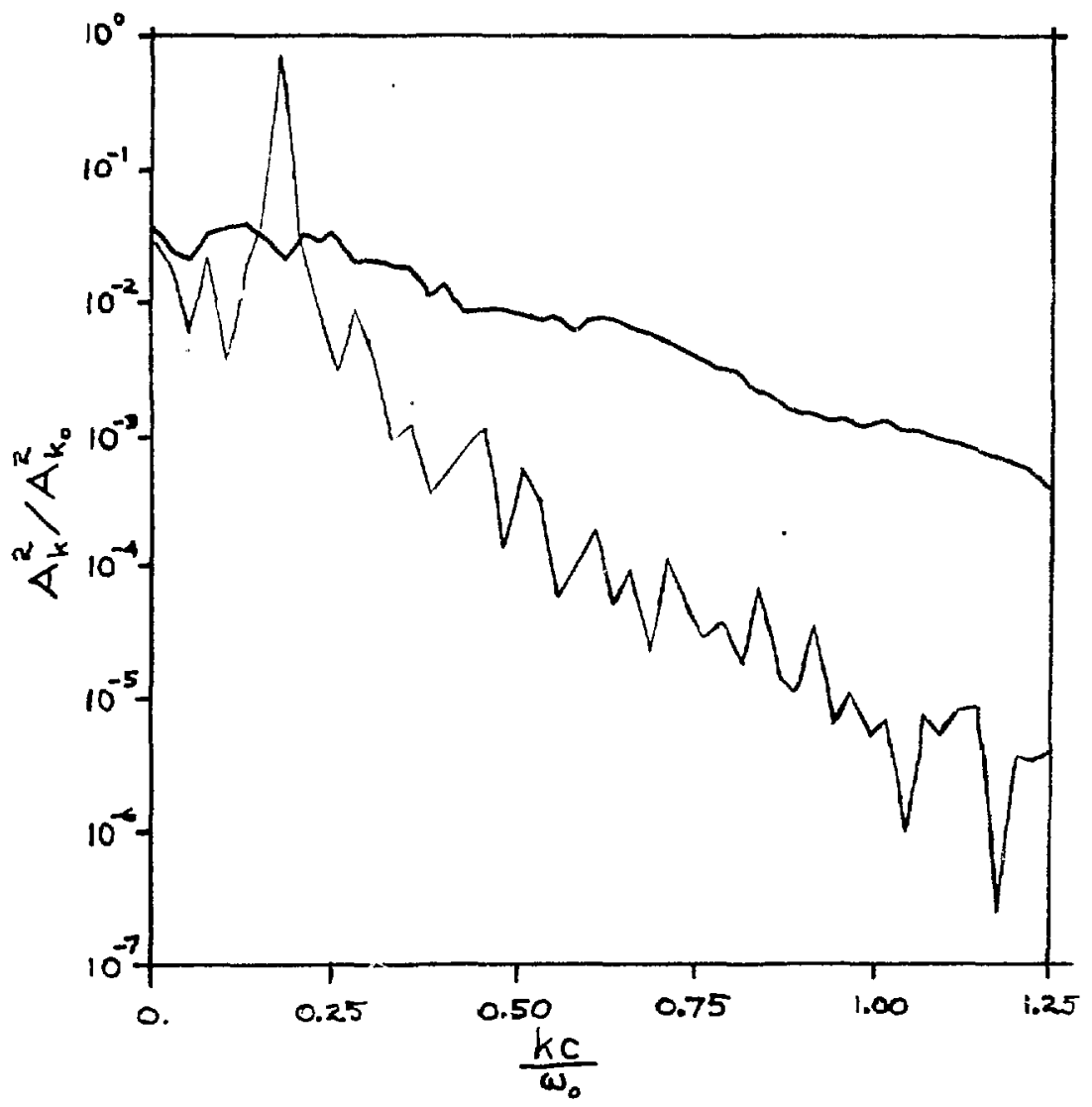

FIGURE 4-10 Time averaged field spectrum; initial spectruto is shown in red while final spectrum to the same scale is in black. 


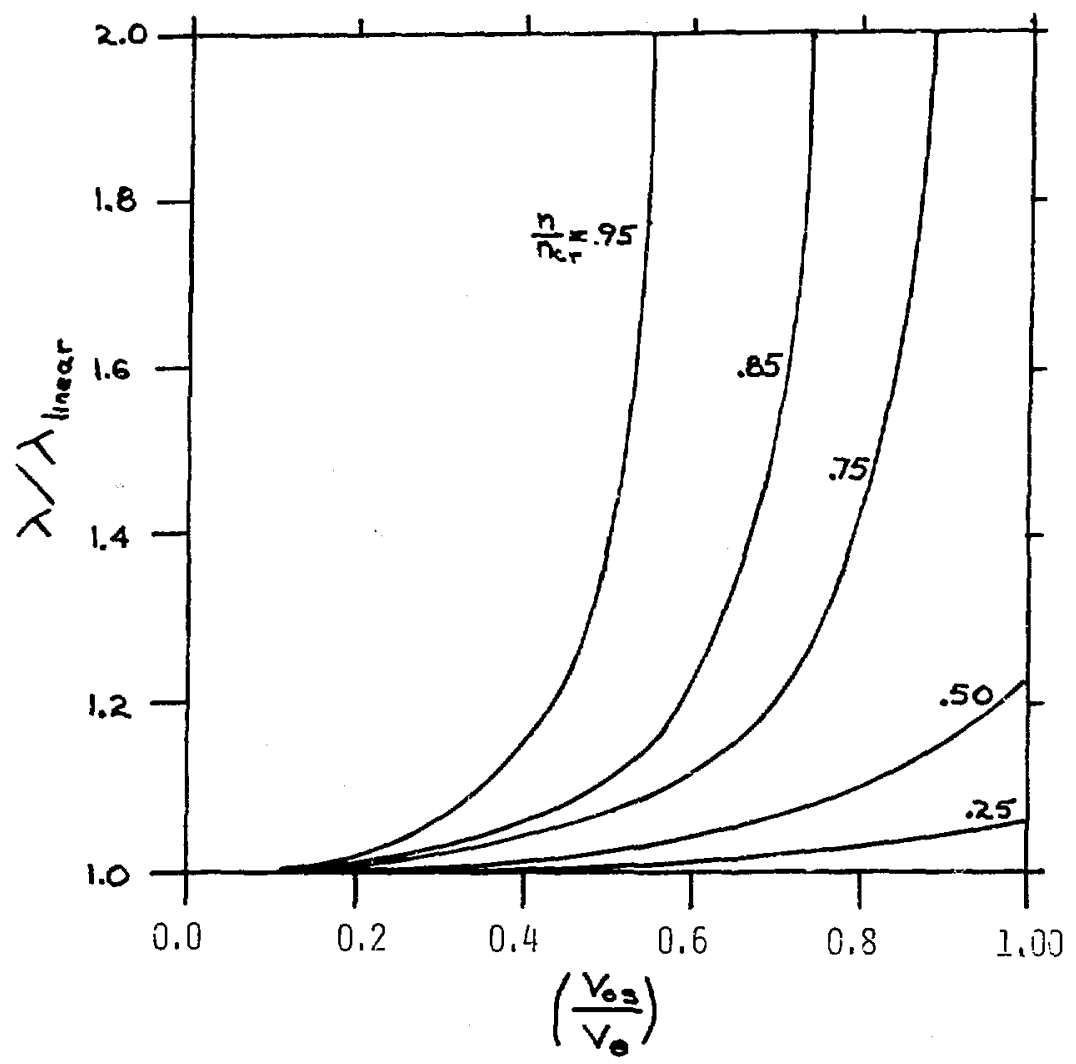

FIGURE 4-11 Wavslength of cn-function as a Eunction of vacuum field strength, for various densities $\mathrm{n}_{0}=.25, .50$, $.75, .85, .95 \mathrm{n}_{\mathrm{cr}}$. 


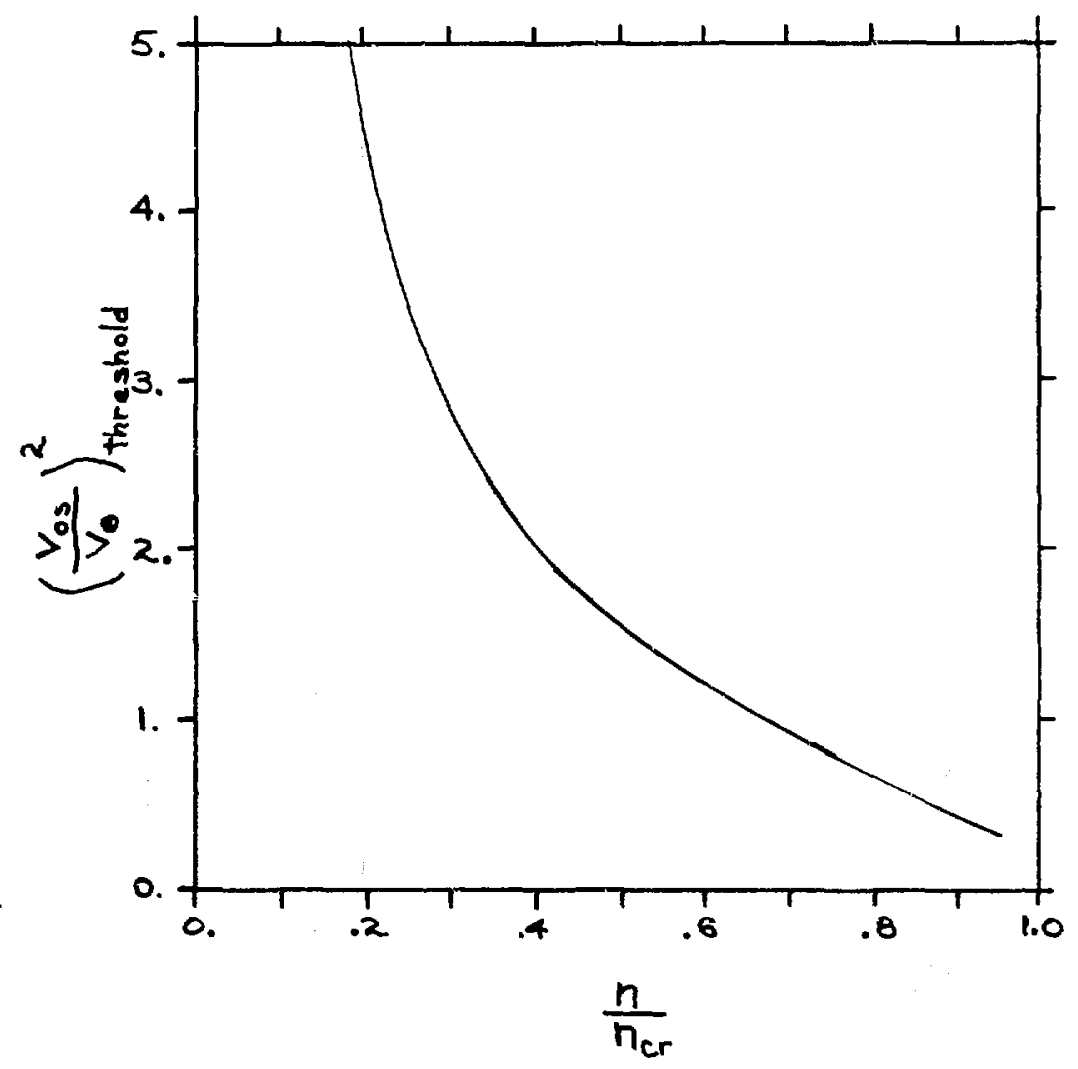

FIGURE 4-12 Threshold field strength For hole formation as a function of density. Threshold criterion is taken to be $m=1$, where $A^{\propto} \mathrm{cn}(x ; m)$. 


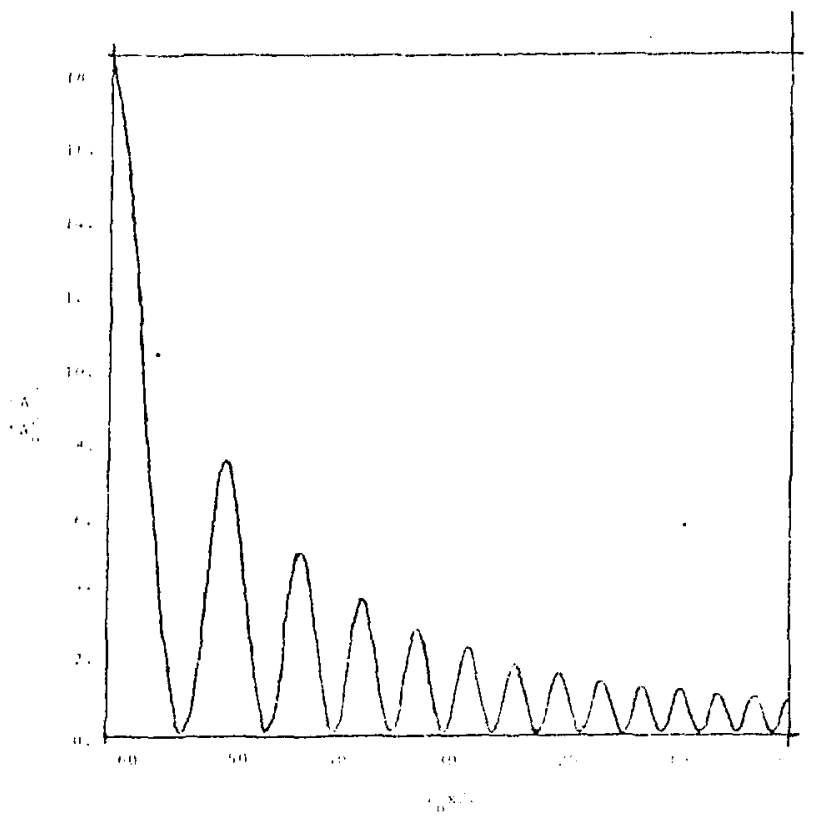

Figure 4-13 Field proite derived from model calculations, $t=100_{0,}\left(l_{H}\right)=548$. 


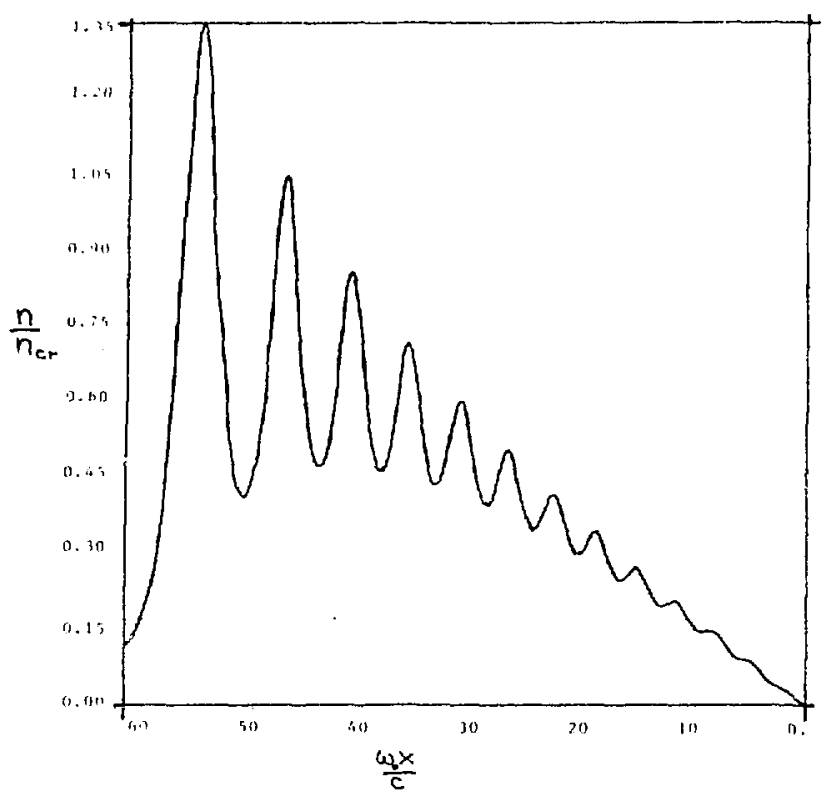

Figure 4-14 Density profile derived from model calrulations for same parameters as figure 4-13. 


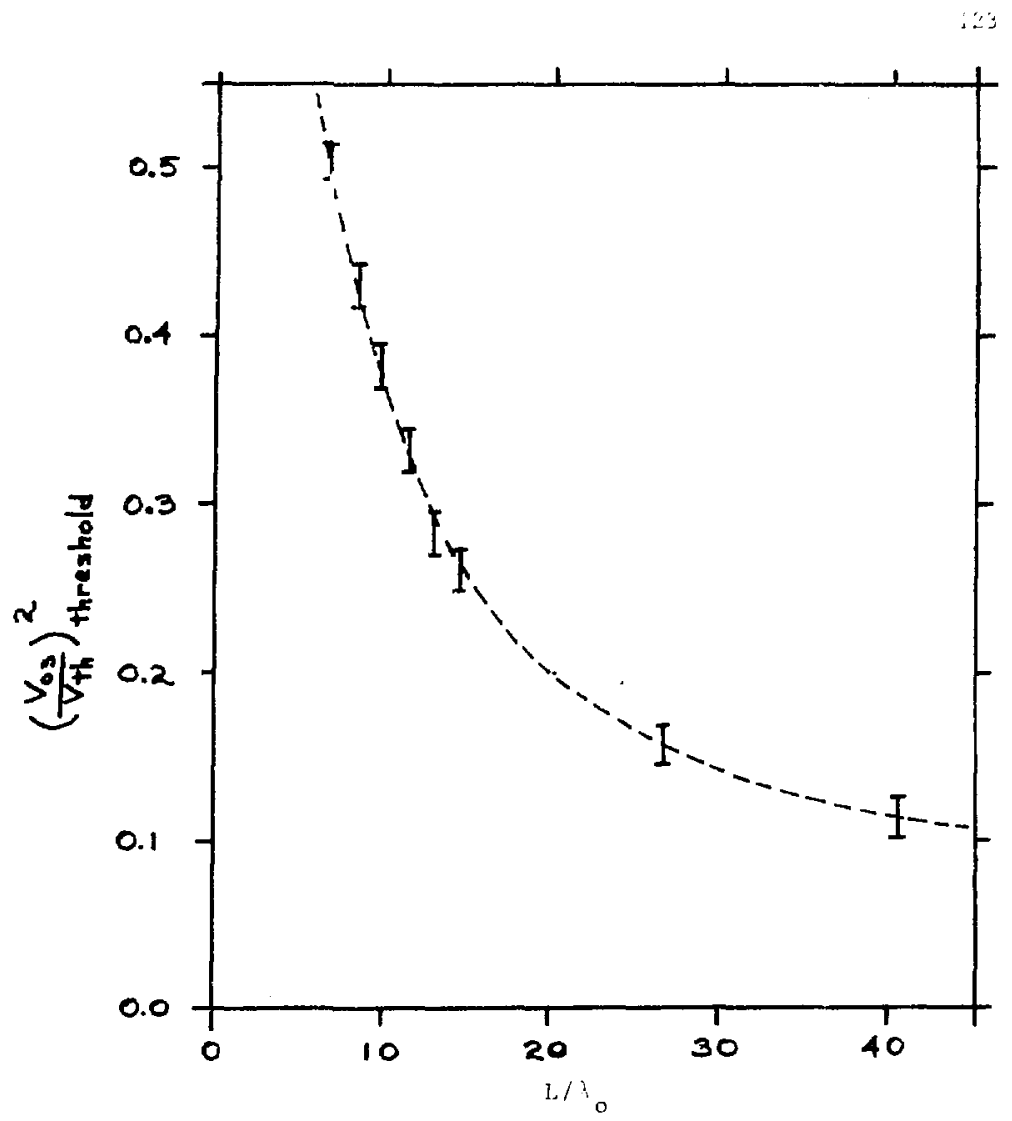

Figure 4-15 Intromogeneous threshold for vacuum hole formation as a function of system length, derived from model calculatiors. 


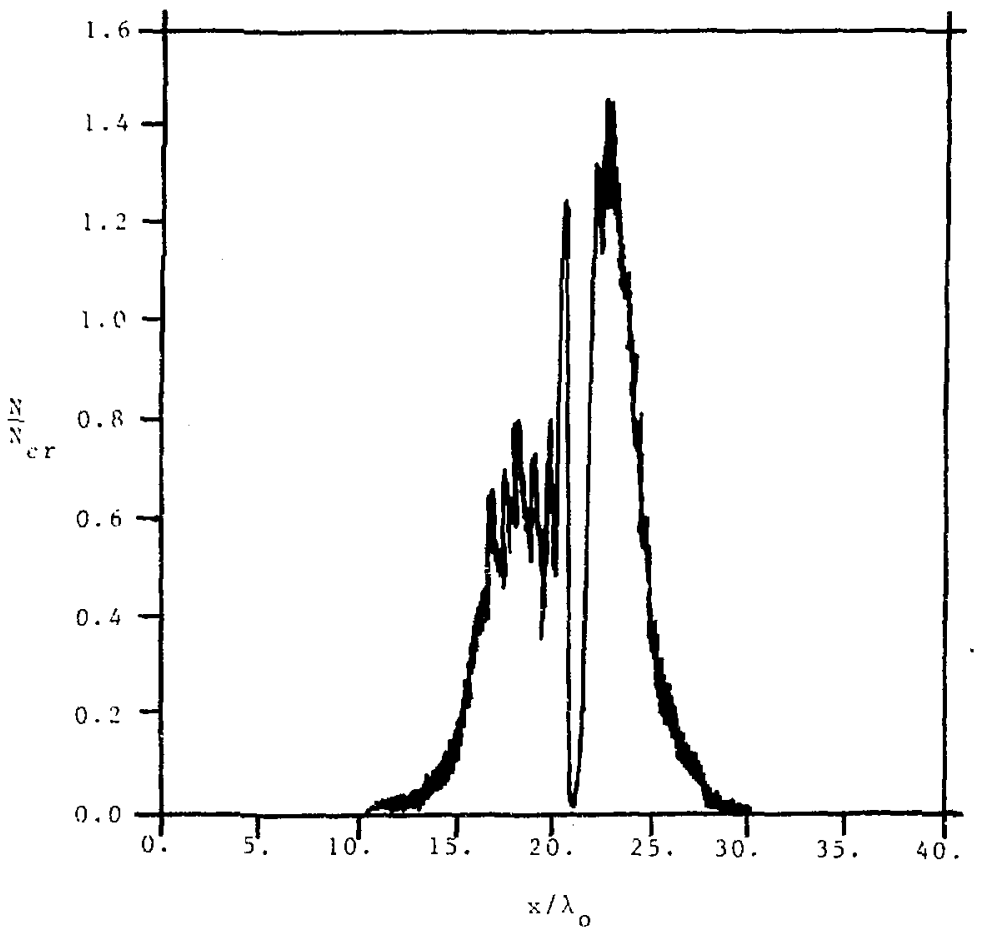

Figure 4-16 Density profile from particle simulation showing late time hole characteristics, $\mathrm{L}_{0}=8 \lambda_{0}$.
$\mathrm{n}^{2}=1.0, \mathrm{~m}_{\mathrm{O}} / \mathrm{M}_{i}=0.01$. 
CHAPTER 5

RDF Study of Absorptive Trocesses in Laser Plastias

In the preceding chapters, the reduced description foralisr. has been applied to stimulated scattering, steepening of the piasma near the critical dorsity, wave trapping, and linecr swelling, factors under a varfety of conditions. Discussion of dissipative processes has been conspicuousiy absent. Extensive tumerical investigations have already been conducted to understand collisionless dissipaticn. Moreover, the particle trapping hich characterizes the saturated. state of many of these mechanisms is not directly describable within the FDF context. There are aspects of the absorption problem to wich this method can be proficably applied. Some of these will be presented in this chapter.

The effect of density nodifications on collisicnal absorption is studied in the first section. The rotivation for considerirg this toplc stems from the fairly sensitive density cependence of the collistonal dissipation rate, $i$ e. as density squarea. Gross density modifications near the critical density, such as self-steepening, can significantly reduce the total absorption efficiency. Brillouin scattering can also induce large density modulations, but these teril to reduce absorption by scattering the energy out of the plasosa before it can be absorbed. Self-consistent evaluatinn of the reduction in efficiency must be approached numerically, and an RDF type code is almost ideal for this purpose. Large lagrangian MrD codes, for 
Erstance, are zoned tco coarsely tc resolve collective effects, and particle simulations do not treat collistonal phenomena accurately. In the second secticn, i tonlinear characteristic of fnverse bremsstrahlung jtote is examined. Quentut mechanical field-dependent corections (i e. nonlinea ) occur in the free-free transition rate. Thest conrespord to multphoton processes and they reduce the overall absorption rate. The llagnitude of the effect is studied by substituling field depondent damping rate for the usual collisional one in the FDF calsulations. The absorption coefficient is found to be significantly reduced for moderate fielo strength (Vos $/ V_{t r} \sim 1$ ), when snelling actors are froluded.

A iew abscrpticn mechonism js discussed in the third section. The tarson-oberan theory, developed in the early 1960's, indicaces that tissipatton occurs when electrons are scattered from finite ion censfly fuctuations, as wel] as from individual fons. Yuch study has been centerer on the resonance which accurs for long wavelength ion flewations. Short wavelengths, on the order of several Debye Lengths however, can alsc give a signiflcant enthancement over colliwefal absrption, espectally if the fluctuations occur between about car-hali crttical denstey and the ritical density itself. Although the strensth of the rectanism depends on the spatial distribution of the in waves, and thetr crientation relative to the field of the Laser efie potentidly groater volume avaizale for absorftion can leat to anconleusiy erficient dissipation. Conitions for the 
generation of such waves are discussed. Finelly, RnF calculations for various ion wave spectra and megnitudes are presented.

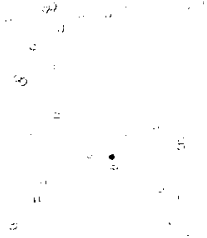




\section{SECTION I}

Keciction in Crilisional Absorption Due to Solf-Consistent Density Nodifications

In Chapter 2, the RDF calculations were used to confirm anclytic pxpressions for collisicnal abserption af laser energy in a linear density profile. Though the thecry was derived in the georetrical cetics Jimit, the exact wave treatreat in the calculations agreed with the theory over a wide rarge of conditions, Nodeling the plasna with a tniform density fratient is a reasonable approximation if the rield strength is much jess than the thermal energy. This corresponds to $\left(\mathrm{os}_{\mathrm{th}}\right)^{2}<\mathrm{l}$ At higher power, hovever, tho results of the previnus chapter indicate that self-consistent density modulatians rust be taken irtc acccunt. In this section we will examine some of the ways self-consistent density rodificaticns can reduce the classical. abscrption.

There are several merdanisrs. ty which density modffications reduce absorpticn. "Irst, thenever $\left(\mathrm{V}_{\mathrm{Cs}} / \mathrm{v}_{\mathrm{thi}}\right)^{2} \mathrm{~L} / \lambda_{\mathrm{o}}>5-10$, significant backscatterfng due to sBs occurs in the underdense refton ${ }^{21}$. The energy in this case ifs simply scatteres out of the plasma. It never reaches high enouph density to experience efficient collisional dissipation. This can be very effective in reducing absorption, since the classical damping rate scales as the square of the density. The group velocity of the light also decreases with increasing density, so the ilght spends more time in the regions of higher dissipation. A second 
mechanism which can lower thit absorption efficiency is self-steepering of the density profile at the critical density ${ }^{8,2}$. The ponderomotive pressure from the laser Irhibits plasma flow at the critical density, the classical turning point, causing an accumulation abcve the critical density and a shelf below it. A density fump there fore forms. Though this mechanism does not inhibit energy from. reaching the high density region, it finds less plasma there, and hence reduced absorption. Finally, propagation of the laser to the critical density and raflection from it leads to a standing wave structure. At low intensity, the pattern takes the form of an Airy function. As seen in the Chapter 4, however, the density ripples which accompany the standing wave can lead to wave trapping and orders of magnitude enhancement over the Airy swelling factor. The peaks of the standing wave pattern occur at the density troughs, though, and this complicates evaluation of the net effect on inverse bremsstrahlung abṣorption.

The reduced description formalism is aloost uniquely suited for such studies. Two dimensional MHD nodeling of laser fusion schemes, for instance, depend critically on energy deposition information. These rely on absorption cross-sections similar to the one derived by Dawson et al. ${ }^{4}$. It is usually prohibitively expensive to resolve any fine structure in the underdense plasma in these calculations, but even a finely zoned problem would miss the first and third mechanisms just discussed. The second, self-steepening, could be observed, since only Intensity magnitude is needed there. Even then, steepening 
due to electrostatic wave pressure vould not be observable. In the plasa:a time scale, farticle simulations can resolve self-consistent olasma effects, but collisjonal phemomena is not accurately represented In such codes. The PDF technique can incorporate realistic collistonal processes, whlle still following the plasma evolution on its own time scale. For the present study, then, it is the logical numerical tool.

$A$ series of calculations performed on a 7.3 wavelength linear protile iliustrate the trends. The electron temperature was not calculated self-consistently in these $r: 5$. The plasma was assumed to be 1scthermal, at $.5 \mathrm{KeV}$. For such short scale lengths, this temperature is in reasonable agreement with hydrcdynamic results. An ion darping rate has included at a level corresponding to a $3: 1$ electron to ion temperature ratio. The electron to ion mass ratio was further set to .01 , to reduce calculational time. Expansion, steepening, and stimulated Brillouin scattering were all 1ritrinsically included in the calculations. The reduction in classical absorption as a function of intensity can be seen in Figure 5-1. At low Intensity, the absorption was well described by Eq (2-5). W'ith increasing fleld strength, hovever, the steady absorption levels decreased monotonically. To show that the points on Figure 5-1, represent true steady absorption levels, the time resolved absorption curves for varlous intensities are plotted in Figure 5-2. Since the total absorption in short scale length calculations increased significantly 
as the plasma expanded, the constant lavels ate evldence tiat SES balances collisional absorption to $\mathrm{a}$ high cegree.

The above calculations shov substantial reduction fin al, : int: s a function of intensity. Since nultiple effects critzitat toward this end, however, further simulations were neces: :ry to evaluate the relative imfortance of each.

Steeprang was evident in , rtain of the above calculictions, though not to the extent predicted, c.f. Ch 2 , Sec III. Competing effects were responsible for at least part of that. As an to in the cases in which $\left(v_{o s} / v_{t h}\right)^{2} \sim .5$, steepening should be strurgly evident but relatively strong SBS under these ccnditions greatly reduced the electromagnetic flux which reached the critical dersity. Thus, both steepening and coliistonal absorpion are affected by SBS in the same qualjtative manner. These two processes can fortunately be separated on the basis of scale length. Erillowin scatrering can be stabilized by sufficiently large inhomogereities, white the steepening, being independent of scale length, should reach the same asymptotic state even in large density gradients. This led to a series of very short scale length calculations, to evaluate the effect of self-steepentng on collistonal absorption efficiency.

The denstty proftle was initialized by allowing an overdense slab to freely expant for several thousani - $t$. The resulting effective scale length was between $1-3 \lambda_{0}$. Although the density jump was measured directly, knowledge of the slope is important for determining the exact intensity structure in the vicinity of the critical 
density. This is clearly important in evaluating collisional

- absorption. Knowledge of the swelling pattern in nonlinear profiles derived in Chapter 2 allowed highly accurate determination of this effective scale length. This in turn was found to be virtually Identical to the slope of the profile at the critical density. The steepening of the profile could therefore be verifled by measuring the decrease in the swelling factor.

Figure 5-3 shows the absorption history curves for various intensities. The profile was initially flattopped at $\mathrm{n}=3.0 \mathrm{n} \mathrm{cr}$. The electron temperature was fixed at $.5 \mathrm{KeV}$, and the lon fluid damping constant was set at $v_{1}=0.0002 \omega_{0}$. As noted in Chapter 2 this form of Ion dissipation can limit free expansion to anomalously low values. The value used here was low enough to permft realistic expansion, but the loss in smoothing allowed too much low density noise. The region around the critical density is less affected. Since the plasma is continuously expanding, the total absorption, as shown in the figure, monotonically increases. The steepening due to even modest fields, however, results in large fractional reductions in the absorption. With the low Ion damping constant, SBS occurred, in even short scale lengths, above $\left(v_{o s} / v_{t h}\right)^{2} \simeq .25$. Monitoring the time averaged intensity pattern verified this unambiguously after $\omega_{0} t=2000$ at that intensity. Ffgure 2-10, at that time, though, shows that profile steepening had already occurred. At later times, the reduction was thus due to a combination of steepening and stimuiated scattering. It is 
in :eresting that a steady absorption level was establisted at higher intensities, despite the profile expansion.

To examine steepening effects at higher intensities, an order of magnitude larger damping constant was used. Although the expansion was nonphysically slow, the total absorption in the shorter system was lower, and the steepening more difficult to resolve, Figure 5-4 shows that the total absorption was still reduced by a significant factor by the density rodifications.

The results of these calculations do not admit ready generalizations. It would be useful to derive expressions for the reduction in classical absorption due to steepening, as a function of intensity. In an expanding profile, however, steepening alone is not sufficient to give a steady absaption level. Even the relative reduction is found to be a function of time. Steepening is clearly capable of causing a significant reduction in the absorption over that of a fixed profile, especially in short scale length plasmas. The quantitative amount of reduction, though, depends on the rate of expansion, the rate of absorption, and the degree of steepening at the critical density. In previous studies of steepening, employing both partfcle and hybrid (particles ions and fluid electrons) simulations ${ }^{41}$, much steeper critical density gradients were seen with the particle calculations. The present fluid calculations are ever more diffusive than the hybrid ones. The absorption coefficients measured here should therefore be regarded as upper bounds when steepening near the critical density is present. 
Finally, we consider reductions due to the standing wave component of the field in an inhomogeneous density profile. As shown in the last chapter, a standing wave leads to botil finjte density ripples and nonlinear field swelling. Since the field is peaked in the density depressions, the density squared dependence of fnverse bremsstrahlung could result in net absorption reductions. This must be partially balancred, however, by the enhanced field amplitude. To evaluate this mechonism, two sets of calculations vere performed. The first set consisted or ordinary RDF calculations with $\left(\frac{v_{\text {os }}}{v_{\text {th }}}\right)^{2}=.5, \mathrm{~m}_{\mathrm{e}} / \mathrm{M}_{1}=.01, \mathrm{~L}=10 \lambda_{\mathrm{o}}$, with the temperature varied formally to alter the amount of absorption. Stintulated scattering, self-steepening, and density rippling could therefore interact to lower the absorption coefficient. In the other set of calculations, the flufd equations were first reduced to a wave equation for the ion density fluctuations. The Inhomogeneous density was fritroduced as a fixed background condition, to describe the medium. Stimulated Brillouin scattering in this configuration is almost exactly the same as in the full RDF calculations. The SBS reductions were thus included correctly; no self-consistent density modifications were allowed. The absorption was computed using the fixed initial profile. The results are sumarized in Table 5-1. The discrepancy between the ful1 RDF computed absorption and the wave equation approach amounted to no more than - few percent over the entire range of absorption. We conclude that density rlppling is effectfvely cancelled out by the field swelifng. 
TABLE 5-1

ABSORPTION AS A FUNCTION OF EIECTRON TEMPERATURE

Linear Denstty Gradient

$\mathrm{L}=10 \lambda_{0}, \quad\left(v_{\mathrm{os}} / v_{\mathrm{th}}\right)^{2}=.5$

$m_{e^{i M}} \quad 0 . \rightarrow$ (Theory) $\quad 0.01$ (F1uid Eqs) 0.01 (Wave Eqs)

$\theta_{e}(\mathrm{KeV})$

0.10

$92.52(95.69)$

89.44

90.00

0.25

$54.00(54.87)$

27.78

29.20

0.50

$24.40(24.52)$

9.62

10.32

1.00

$9.46(9.47)$

4.11

4.79

2.00

$3.42(3.45)$

1.57 
SECTION II

Direct Intensity Dependence in Collisionas Absorption

Ic this point, the classical absorption was computed with an intensity Independent coefficient, $\nu_{\mathrm{em}}$. This is tantamount to considering enly single photon interactions. Several authors $42,43,44$, however, have shown that the correct quantum mechanical result includes both the themel and the nscillating components of the electron velocity. Silin ${ }^{45}$ has furthemore derivec the same result from purely classical arguents. Since the oscillating velocity is proportional to the field anplitcde, $v_{\text {os }}=\mathrm{eE} / \mathrm{rw}$, the local value of the fielc itself plays e direct role in the clessical absorptive process.

i.et us examine the magnitude of this intensity dependence. In the limit that $\left(v_{o s}\left(v_{t h}\right) \ll 1\right.$, the full expression reduces back to Eq $(2-i)$. In the other limit., $\left(v_{o s} / v_{t h}\right) \gg l$, silin gives

$$
v=v_{0} \frac{12}{\sqrt{2 \pi}\left(\frac{v_{o s}}{v_{t h}}\right)^{3}}\left\{\ln _{n}\left[\frac{1}{2}\left(\frac{v_{o s}}{v_{t h}}\right)\right]+1\right\}
$$

For calculational purposes, we have fit the exact expression with

$$
v=v_{0}\left(1+\frac{1}{3}\left(A^{2}\right\rangle\right)^{-\frac{3}{2}}
$$

In the weak field limit, this of course gives the usual result, and in the strong field linit, it is within ten percent of the expression 
given in Eq (5-1). Note that the tire averaged fieli, calculated, is used to evaluate $(5-2)$. For a traveling wave fielc, the everage velue of the field at a given density is approximately

$$
\sqrt{\Gamma^{2}}=\frac{1}{\sqrt{2}} E_{0}\left(1-\frac{n}{n c x}\right)^{-1 / 2}
$$

The intensity dependence is therefore a strctig effect crly for ficids such that: $\left(v_{o s} / V_{t h}\right)>1$. The picture is dranatically cifferent for the nore realistic starding wave node. Ther the tire averagec patter: is an Airy function, normalizec to $2^{1 / 2} \mathrm{r}_{\mathrm{os}} / \mathrm{r}_{\mathrm{th}}$. Fieli effects are localized, but at locations whele the field j.tself is largest. The Airy shelling factor, morecver, Jeads to proportionally lägest reductions in the ccllisional dissipation rate near the critical dersity. The reduction in collisional danping in a stending have pattem can be significant at Jower intensities then previously suspected. A series of salculations done in a fixec linear dersity profilet confirm the qualitative ideas advanced above. Figure 5-5 shows absorption history curves at varjous vacuum field strengths. The electron temperature was fixed at $.5 \mathrm{KeF}$, which gives forty-four percent absorption in a $20 \lambda_{0}$ scale length, for $\lambda_{0}=10^{-4} \mathrm{cn}$. The actlia! abscrption is reduced by a factor of 2 for $\left(\mathrm{v}_{\mathrm{os}} / \mathrm{v}_{\mathrm{th}}\right)^{2}=1.0$. For $\left(V_{\text {os }} / v_{t h}\right)^{2}+4$, the absorption talls bolow the classical. figure by a factor of $4-5$. Figure 5-6 shows the total absorption coefficient for these conditions as a function of field strergth. 
To evaluate the reduction in inverse bremstrahlung due to intensity cependence of the cross-section, consider the simpler model prebler of absorption cf a standing wave in a homogeveous plasma. If t) . steady part of the absorption coefficient is denoted by $\mathrm{r}_{0}$, the absorption integrated over one wavelexgth is

$$
\operatorname{ABS}=\frac{1}{k} \int_{0}^{2 \pi} c_{0} \sin ^{2} y d y
$$

vh: $=$ re $y=k x$, and $A B S=-\frac{\mathrm{dA}^{2} / \mathrm{dx}}{\mathrm{A}^{2}}$. The integral is easily evaluated to $g i: e$

$$
A B S=\pi C_{0} j k
$$

No: suppose o has the following spatial, $i \in$. intensity, dependence

$$
G=\sigma_{c} /\left(1+\frac{1}{3} A^{2} \sin ^{2} y\right)^{3 / 2}
$$

The integrated absorption then becomes

$$
\operatorname{ABS}_{1}=\frac{4}{k} \int_{0}^{\pi / 2} \sin ^{2} y d y /\left(1+\frac{k^{2}}{3} \sin ^{2} y\right)^{3 / 2}
$$

With a little help fror standard integral tables (c.E. Gradshteyn and Byzhik, Ens. 2.584 and 2.598), this integral can be expressed in terms of elliptic integrals, 


$$
A B S_{1}=4\left[\frac{A^{2}}{3}\left(1+\frac{A^{2}}{3}\right)^{1 / 2}\right]-1(K(a)-E(a)) G_{0} / K
$$
where $a=\operatorname{sir}^{-1}\left\{\frac{A / 3^{1 / 2}}{\left(1+A^{2} / 3\right)^{1 / 2}}\right\}$. In the linit that $A+0$. it cen $b E$ easiiy verified that $(5-7)$ reduces ton (5-5). On the ocher hanc, when $A \gg 0, A B S_{1} \alpha A^{-3}$, which can be a significant ridrtion. A reasurt of the reauction catn be gained by computing

$$
f=A B S_{1} / A B S=\frac{4}{\pi}\left[\frac{A^{2}}{3}\left(1+\frac{A^{2}}{3}\right)^{1 / 2}\right]^{-1}(K(a)-E(a)) .
$$

For a standing wive we will take $s^{2}=2 v_{o s}^{2} / v_{t h}^{2}$, although this should be mul tipliec by $\left(1-\frac{n_{0}}{n_{c r}}\right)^{-1 / 2}$ to account for swelling. These restilts are displayec in Table 5-2.

In an inhomogeneous piasna, both the field structure and the abscrption integral are more corplicated. He nay obtain an esticate of the exact reduction, however, by considering the swelling, in an Airy pattem. Th.e low amplitude absorption integral in a linear density gradient was evaluated in Chapter 2, Eq (2-5). Exactly the sace absorption would have cccurred in a homogenecus plesma if $G_{0}=\frac{32}{15}(\mathrm{ct})^{-1}$. The difference between the homcgenenus analysis and. abscrptior in an Airy pattem is that the reak vaiue of the fielc, $\hat{f}^{2}$, swells neaz the critical density. The effertive absorpticn therefore will be given by (5-7), for an averaged value of the peak fielc. Though sophisticated averages could be performed, a reasonahly accurate estrate results from simply averaging the largest and smaliest lobes of the Airy fattern. These f-factors are compared with 
TNBLE $5-2$

Prraentage Reduction in Inverse Bremsstrahlung Absorp:ion as A Function of Field Strength

$$
A^{2}
$$

$\sin (a)$

⿷

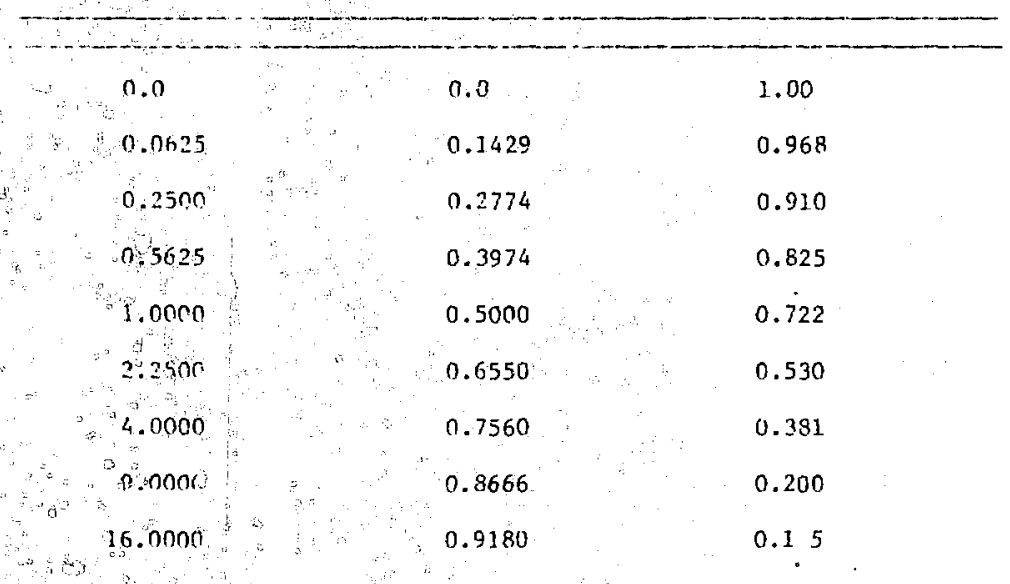


simulation results in Figure 5-?, for $L=4.8 \lambda_{0}, E_{e}=.5 \mathrm{KeV}$, and $\frac{m_{e}}{M_{I}}=0 .$, the low amplitude absoption is $12.5 \%(Z=1)$. This averaging procedure glves astontshingly good agreement.

For compartson purposes, a traveling wave fossesses no spatial structure, and a time averaged value of $A^{2}=.5\left(1-\frac{n_{0}}{n_{c r}}\right)^{-1 / 2}\left(v_{o s} / v_{t !}\right)^{2}$. Integrating the absorption cuer one wavelength gives

$$
A B S_{2}=\lambda_{0} G_{0} /\left(1+\frac{A^{2}}{3}\right)^{3 / 2}
$$

and a reduction factor of

$$
f=\left(1+\left(1-\frac{n_{o}}{n_{c r}}\right)^{-1 / 2}\left(v_{o s} / v_{t h}\right)^{2} / 6\right)^{-3 / 2}
$$

This f-factor is $1 \mathrm{n}$ very satisfactory agreement with simulations.

The fractional reduction in absorption, was observed to be insensi"Ife to density scale length. A serfes of calculations were performed with $\left(v_{o s} / v_{t h}\right)^{2}=1.0, \mathrm{e}_{\mathrm{e}}=0.5 \mathrm{KeV}$, and $\mathrm{m}_{\mathrm{e}} / \mathrm{M}_{\mathrm{i}}=0.0$. The following scale lengths were calculated: $L / \lambda_{0}=21.0,16.4,13.2$, 10. $3,8.6,6.5$, and 4.8 . Though the magnitude of absorption viaried by almost a factor of four over these parameters, the f-factor changed by only three percent, $1 \mathrm{e}$. from .43 to .40 . Figure $5-8$ shows the Intensity dependence of absorption in two profiles, $L=21 . \lambda_{0}$ and $L^{\prime}=4.8 \lambda_{0}$. There is clearly, little difference in the fractional reductions. 
Cn the bisis of the above results, we conclude that the total abscrption can be modeled satisfactorily by

$$
\Lambda=1-e^{-K_{T} f}
$$

where $k_{T}=\frac{32}{15} \frac{T}{c \tau}$, and $f$ is given by Eq (5-8), with $A^{2}$ appropriateiy weraged over the intensity pattern in the plasma. The significance of this simple theory is that it relates the intensity dependent abserption cuefficient to the linear Airy function, suitably modified by iactors such as were treated in chapter 2 .

The results of these two sections should be briefly sumarized. As expected SBS proved capable of reducing the collisonal absorption rate significantly. Self-steepentng was difficult to observe in mederate scale length plasmas due to the flux reduction caused by SBS, but in sufficiently steep gradients, absorption reductions on the order of 2 were measured ing RDF calculattons. Thêre was enough numerical uncértginty in these results that general prescriptions for the effect were Ampossible to derive. Numerical diffusion associated wth the Eulerian algorithm undcubtedly smoothed the profile more than it is realistically, however, so the calculated results probably overestimated the total absorption. There are indications from particle simulations that the real profile at the critical density has a steeper gradient than the Ror calculations. 
Finally, inclusion of another

effect fn the calculaticrs, Intensity dependent inverse bremstrahlung rate, jielded very significant reductions in the absorption at modest fleld strength. The factor is strong enough to swamp thermal effects for $\left(V_{c s} / v_{t h}\right) \gg 1$. The ramifications to the problent of cold plasma heating uay be enormous. It is conventionally assumed that a cold dense plasma absorbs laser radiation so efficiently that it rapidly heats to several hundred $\mathrm{eV}$, after whlch the plást. begins to thermally bleach. If the rise tfme of the laser f.s sufficiertly fast, however, $\left(\mathrm{V}_{\mathrm{os}} / \mathrm{v}_{\mathrm{th}}\right)^{2}$ can be very large number. Clearly, $(5-2)$ then predicte that the plasma temperature is irrelevant, and that the collisional absorption is reak. Only if the laser intensity is increased slowly enough so that $\left(V_{c s} / V_{t h}\right) \leq 1$ wll1 the converitional heating history be valid. of course, this does not imply that the plasma will remain unheated. With such a large value of $\left(V_{o s} / v_{t h}\right)$ and a relatively weak collisional absortion rate, parametric instabllitles may well fulfill the role of heating the plastia up to a few hundred electron volts. Shocking up of the critical surface could then spcil these processes for the duration of the laser pulse, but the turbulent heating is rapid enough that they would have already served a purfose. In any case, self-conslstent interaction of many presesses could well depend on the initial mode of heating.

In these sections, various processes were dischised which tended to decrease the inverse brensstrahlung efficlency. The next"section deals with a new method for increasing the overall efflciency. 


\section{SECTION I I I}

Enhancement of Laser Absorption with Short Wavelength Ion Fluctuations

Wost collective absorption processes are limited to the imaediate vicirity of the critical surface. Self-steepening, cavitons ${ }^{47}$, and bubbles, however, not only inhibit collisional absorption, they may kradient stabilize the collective processes. Uncertainty about the critical surface structure, therefore, has lead to renewed interest in wechanisms capable of absorbing the energy over a brcad density range. Collisionel or inverse brensstrahlung is one such mechanism, but in the previous section, it was shown how its efficiency can be severely legraded by self-consistent density or intensity effects. In this section, she th wavelength ion fluctuations will be examined, and shown to result in significant enhancements over collisional absorption.

Dawson, oberan, and Ron ${ }^{48-50}$ showed that finite density correlations can enhance the absorptivity of a plasna. Collisional absorpLion consists of the scattering of oscillating electrons from massive ions. The Dawson-Obertan theory predicts enhanced absorption when electrons are scattered off fon waves. Consicier a spectrum of ion flictuations such that $n_{s}(x)=\sum_{k} n_{k} e^{i k x}$. Transposition of the electrons from their equilibrium positions by an external electrlc field will result in a finite ejectrostatic field in the plasma. Fourier transformation of the continuity equation and Poisson's equation, for a unform external field, gives 


$$
\begin{gathered}
n_{s}(\omega)=-i \frac{\bar{V}_{o s}}{w} \cdot \nabla n \\
1 \bar{k} \cdot E_{L}(k, \omega) \bar{E}_{k, \omega}=-4 \pi e n_{s}(k, u)
\end{gathered}
$$

where the oscillating electron veloctty is given by

$$
v_{\text {osc }}=-\mathrm{eE}_{\text {laser }} / \mathrm{tr \omega} \cos (\omega t)
$$

where $\varepsilon_{L}(k, w)$ is the dielectric constant function. The average power dissipated is just given by $\bar{J} \cdot \bar{E}$. By comparing this with the laser fleld energy, one can then define an effective collision frequency. The details are included in Appendix D. A given density fluctuation spectrum at an angle $\theta_{k}$ to the laser E-field,

$$
\nu^{*}=\frac{\omega}{2}\left(\frac{\Delta n}{n_{c r}}\right)^{2} \sum_{k} \frac{\operatorname{Im}\left(\varepsilon_{L}\right)}{\varepsilon_{L}^{2}} \cos ^{2} \theta_{k}
$$

where $\Delta n \equiv\left(\sum_{k} n_{k}^{2}\right)^{1 / 2 *}$. Figure 5-10 shows the qualitative effect of spectral width for $\mathrm{k} \lambda_{\mathrm{D}}=.5$, when $\Delta \mathrm{n}=.07$.

The above discussion tacitly assumed that the ion fluctuations would be distributed over the entire density profile. Since the actual distribution depends on the mechanism which creates the waves, some discussion ó posstible wave sources is needed. One of the most attractIve, and simultaneously most daming, characteristics of short wavelength ton enhanced absorption ts that the waves themselves are not a * The fluctuation spectrum must be "flat-topped" for this form to be true. 
consequence of the abscrption. They are secondary laser plasma effects, created as a byproduct of more fundamental interactions. Within the category of "mcre fundamental" one should include free expansion and conduction of energy into the overdense plasma. As the following will show, both of these can become fion wave sources, though it is not clear that either will be useful in this context. High energy ions, cavitons, parametric instabilities, and streaming across self-generated magnetic fields are all more esoteric mechanisms, but under given conditions, these could be realist1c sources.

F1rst, conduction of heated electrons into an overdense plasma must be balanced by a cold return current. This can he unstable with respect to an ion-electron streaming instability. The associated ion acoustic waves can be broad in angle and at levels of $\left(\frac{\delta n}{n_{0}}\right)^{2} \sim 10-30 \%$, commensurate with trapping saturation. Uniortunately, simulations of this process indicate it occurs at considerably greater than critical density, 1 e. $\mathrm{n} \simeq 5 \times 10^{21}-10^{22} \mathrm{~cm}^{-3}$ for $\mathrm{n}_{\mathrm{cr}}=10^{21} \mathrm{~cm}^{-3}$. The density fluctuations must be in the underdense plasma before they can contribute to the absorption. Let us consider an example to test the plausibility of thts.

Suppose $\lambda_{\mathrm{o}}=1 \mu \mathrm{m}, \mathrm{L}=20 \mu \mathrm{m}, \mathrm{n} \alpha \mathrm{r}^{-3}, \theta_{\mathrm{e}}=2 \mathrm{KeV}, \theta_{\mathrm{e}} / \theta_{\mathrm{i}}=10$, and that the waves are generated at $n=5 \times 10^{21} \mathrm{~cm}^{-3}$. The wave source is therefore somewhat more than $10 \mu \mathrm{m}$ from the critical surface. If a hydrogenic plasma is assumed, the ion acoustic speed is $\mathrm{c}_{\mathrm{s}} \doteq .0015 \mathrm{c}$, and the time for such a wave to propagate to the critical surface is $\Delta t=2 \cdot 10^{-3} c_{s}$. Even a $10: 1$ temperature ratto w11l result in 
huge Jandau damping in this tine, however. For any vestige of the orfginal wave to reach the critical surface, the wave source must be within about 5 m of that point. Aside from casting doubt on the return current streaming instabizity as an appropriate ion wave source, this exercise also Indicates that ion waves are unlikely to propagate far from their generation location.

The last point is crucial in evaluating plasma expansion as a fluctuation source. In an electron-ion plasma, the more mobtle electrons set up an ambipolar potential which drags the icns along. If two ion species are present, each will receive an erergy $z_{i}$ e from the potential. A velocity differential can thus be established

$$
\Delta v=\sqrt{\frac{2 Z_{1} e_{\phi}}{M_{i}}}\left\{1-\sqrt{\frac{Z_{2} M_{i}}{Z_{1} M_{2}}}\right\}
$$

where $M_{1} \leq M_{2}$. These two "beams" will equilibrate in a time 51

$$
\tau_{1 i} \doteq \frac{M_{i}^{2}(\Delta V)^{3}}{4 \pi e n_{2} \ln \Lambda z_{1}^{2}\left(2+\frac{M_{i}}{n_{2}}\right)}
$$

If $\tau_{i i} \ll \frac{V_{1}+V_{2}}{2 i}$, the beans will be collisionally tied together; that is, they will not expand at different speeds. If $\Delta \mathrm{V}>\mathrm{v}_{\text {thi }}$, however, an Ion-ion streaning instability can occur. Although simulations show that the expansion potential can drive such an instability in a collisionless plasma, the process occurs primarily in the low density region of the blow-off. Unless the fon waves created in the 
low density region can propagate closer to the critical surface, they are unlikely to be useful in laser absorption.

Although the potential accompanying the expansion may not be useful for these purposes, the one found near the crttical surface due to efther self-steepening or resonant abscrption may be 1deal. In the first place, this potential can be much stronger than the ambipolar one. Second, it is ideally lacated, for instabllities which can generate the necessary fluctuations are al ready at the desired densties. Finally, streaming instabilities which orfglnate near the critical density, drivan by an outward propagating bean, could concelvably generate short wavelength fluctuations throughout the profile. This mechanism has already been observed in the microwave experiments of Wong, et al. ${ }^{52}$, in which fluctuations of $\frac{\delta n}{n_{c}}=5 \%$ were seen. Since 1 t was the fast ion bunch which generated the waves, and since sL-h bunches are often neasured in high power laser/plasma experiments, it is at least plausible that short wavelength ion waves are present in such experiments. Other mechanisms, such as streaming across self-generated magnetic fields, could also be discussed, but the above discussion is suggestive enough to show how ion waves may be created in laser fusion plasmas.

Once the ion spectra exists, it is proper to inquire how it. affects absorption. The magnitude of $v^{*}$ is 2-5 times greater than the collisional damplng rate in the low intensity limtt, depending on the electron temperature. As Figure 5-11 shows, it possesses a significant increment over a wide censity range, down to roughly 
$.5 n_{c r}$ for $k \lambda_{D} \doteq .5$. The exact magnitude, however, can vary widely in a real experiment, due to the cosine squared factor in Eq (5-14) which depends on the angle between the fon waves and the laser electrlc fleld vector. For lack of accurate distribution information, we have averaged over a presumed isotropic fluctuation spectrum. This reduces the magnitude by a factor of between 1.5 and 3 , depending on the laser polarization.

Using an angle averaged reduction factor of .5, Eq 5-14 was evaluated for a 20 wavelength linear density profile, and Included in a series of RDF calculations. Collistonal absorption was also included. The absorption from this classical effect alone was eighteen to ninteen percent for $\epsilon_{\mathrm{e}}=1 \mathrm{KeV}$. With a uniform distribution of ten percent density fluctuations, the absorption rose to over ninety percent, for this scale length. The large entiancement is due to a modest increase in the local dissipation rate, but extended over thany wavelengths.

Intensity dependence was included only through SBS. The stimu:lated scattering, however, reduced the classical absorption to sevea to elght percent for $\mathrm{L}=20 \lambda_{0}$ and $\left(\mathrm{V}_{\mathrm{os}} / \mathrm{V}_{\mathrm{th}}\right)^{2}=.50$. Since Brillouin scattering is representative of scattering type instabilities and because its behavior is moderately well understood in one dimension, it is illustrative to consider its competition with the enhanced absorption. The extended character of the fluctuation induced mechanisu is in contrast to other absorptive processes, such as those near the critical density. Brillouin scattering occurs at lower density 
than those frocesses, and so evolves independent $1 \mathrm{y}$ of then. For density fluctuation enhancements in the absorption, a large density overlap exists with SBS. The effect of the latter can therofore be evaluated by performing the self-consistent calculations. For a $20 \lambda_{0}$ scale length, the intensity dependence on absorption is indicated by Figure 5-12, for $\frac{\delta n}{n_{c r}}=7 \%$. The calculations were extended only up to $\left(V_{o s} / V_{t h}\right)^{2}=1,0$, for which very large SBS would be expected in these conditions. Even at this field level, however, almost thirty-five percent absorption occurred.

Another series of calculations exanined the effect of the magnituie of fluctuations. Figure 5-13 shows the absorption as a function of $\frac{\delta n}{n c r}$ for a $20 \lambda_{0}$ fixed density profile. The absorption due to collistons alone was again eighteen percent. When the profile was allowed to relax, $i$ e. finite ion mass $\left(m_{e} / M_{i}=.01\right)$, Erillouin scattering was again posstble. In the classical case this reduced absorptivity to less than eight percent. In Figure 5-13, though, the absorption can be seen to remain well above this level, even for $\frac{\delta n}{n_{c r}} \doteq 3 \%$. The fractional reduction is also reduced from the collisional case, as comparison with Figure 5-1 shows.

Finally, the fact that SBS scales roughly as $\left(\mathrm{v}_{\mathrm{os}} / \mathrm{v}_{\mathrm{th}}\right)^{2} \mathrm{~L} / \lambda_{0}$ indicates that scale length effects may cancel when the enhanced absorption mechanism is competing with stimulated scattering. Although conclustve results have not been sbtained, the scale length dependence of absorption 1 a series of simulations with $e_{e}=1 \mathrm{KeV}$, $\left(v_{o s} / v_{t h}\right)^{2}=0.5, m_{e} / M_{i}=.01$, and $\frac{\delta n}{n_{c r}}=.07$ at $k \lambda_{D}=.5$ indicate 
relative insensitivity to the length. Figure 5-14 shows these results. Since actual length effects are expected to be more cormplicated than the one dimensional calculations, it is not clear that Figure 5-14 has practical applications. Two dimensional simulations, RDF or otherwise, are more appropriate for addressing such problems.

The RDF simulations overall show that modest short wavelength ion fluctuation levels can result in twenty to fifty percent absorption efficiency. Once the fiuctuations are created, they give absorption which is relatively resistant to self-steepeni:z or SBS depredations. One dimensional electrostatic particle simulations with a fixed density spectrum, moreover, confirm the theoretical dissipation magnitude, Eq (5-14), and yield a "soft" heated electron distribution. Suprathermal taiis are conspicuousiy abseid. There are still many uncertainties in applying this mechanism to actual experienents. Neither the angular nor spatial distribution of density fluctuations can be astinated accurately. Even the spectrum and nagnitude are only crudely estimatle. The insensitivity to density modiffcations, softness of the heated electron spectrum, and relatively robust Ieve' of absorption, however, make fluctuation induced absorption very attractive for laser fuston. The calculations discussed in this section were based on rasonable estimates of che fluctuation dist:ibution. The promise of these calculations is sufficiently great that further studies are strongly suggested. Two- or three diuensional studies involving oblique and focused beams in arbitrary fluctu tion 
discributions, and two cimensiunal particles simulations to detemine realistic distributions, seem logical extensions of the present work. 


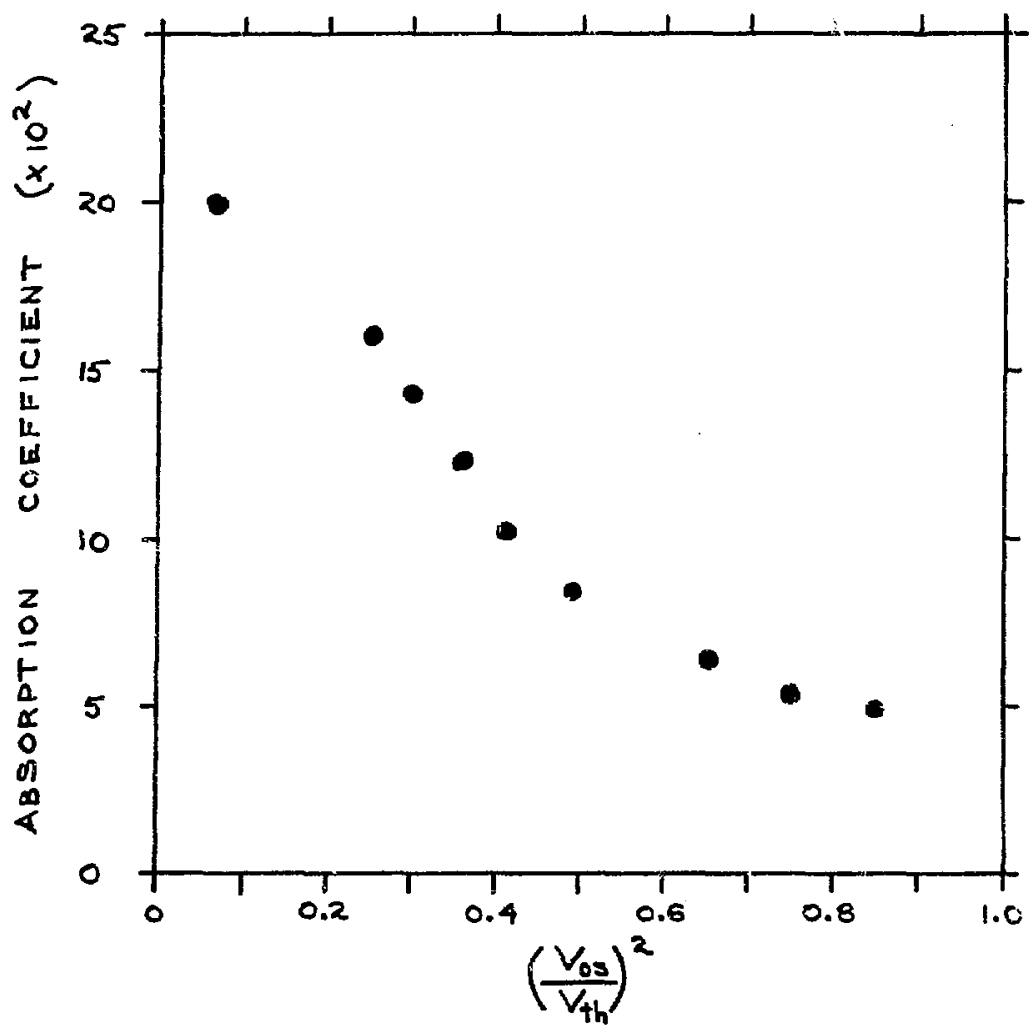

FIGURE 5-1 Collisional Absorption as a function of vacuum field strength. $\mathrm{me}_{\mathrm{e}} / \mathrm{M}_{\mathrm{i}}=01, \theta_{\mathrm{e}}=0.3 \mathrm{KeV}, \mathrm{L}=\mathrm{i} .5 \mathrm{a}:$ $y=.0025 \omega_{\mathrm{e}}$. 


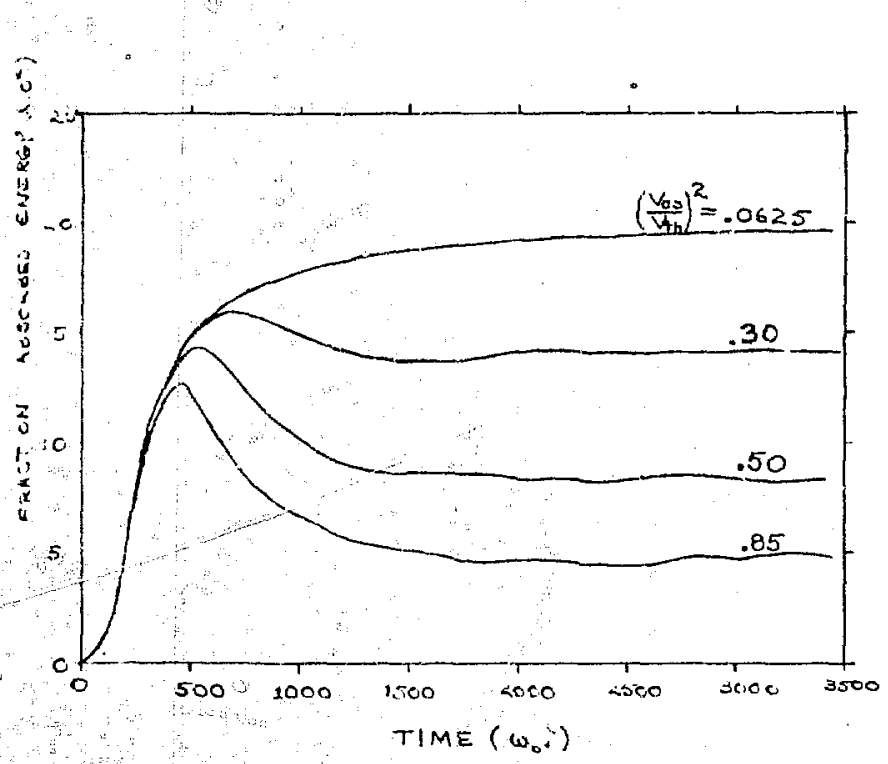

Figures-ansorption as a funetion of line for various iaste ficld strengths, same parameters as in Figure 5-1. 


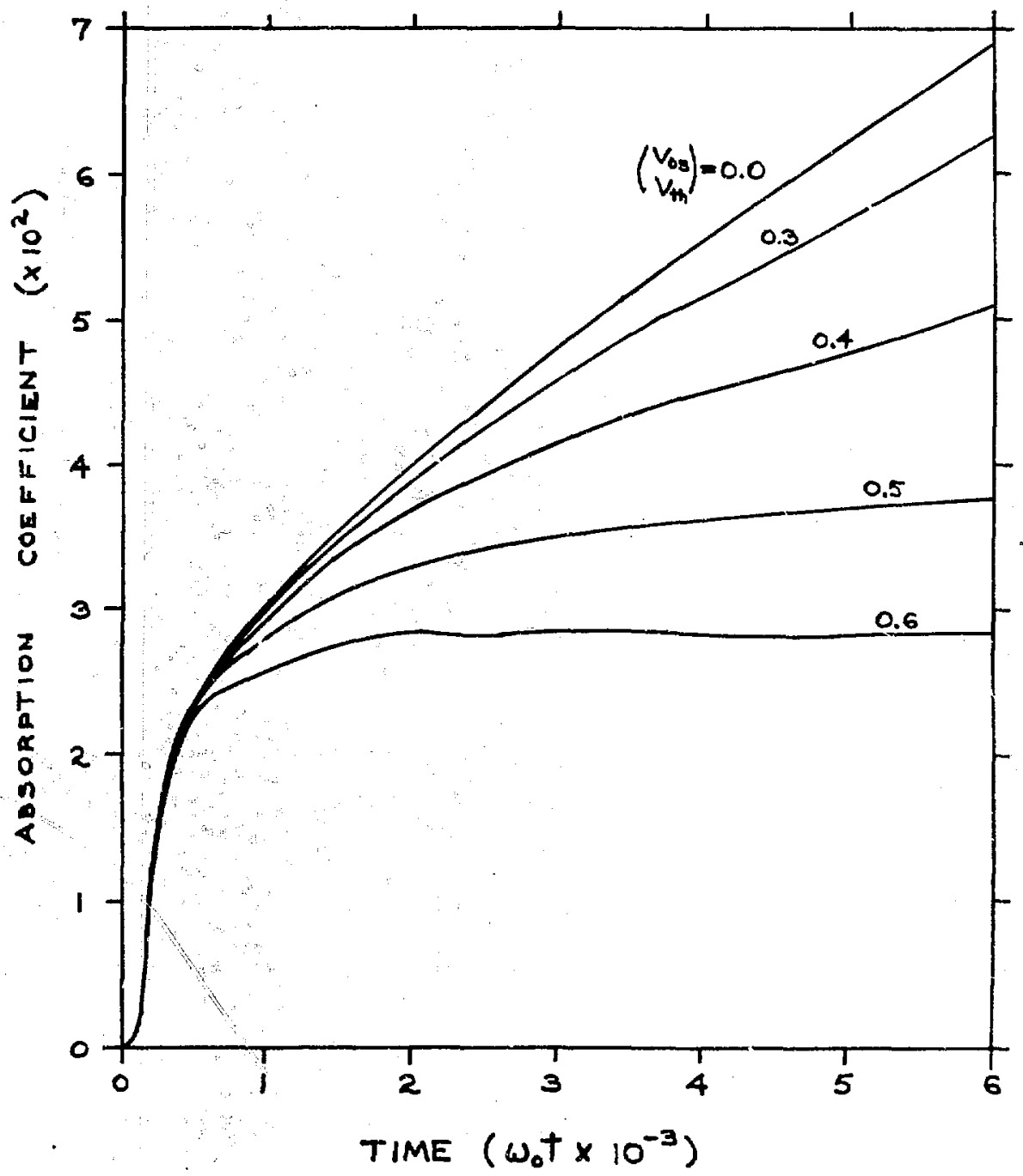

FIGURE 5-3. Absorption histories showing the efrect of seli-

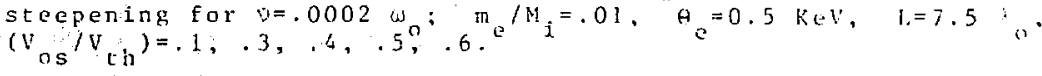




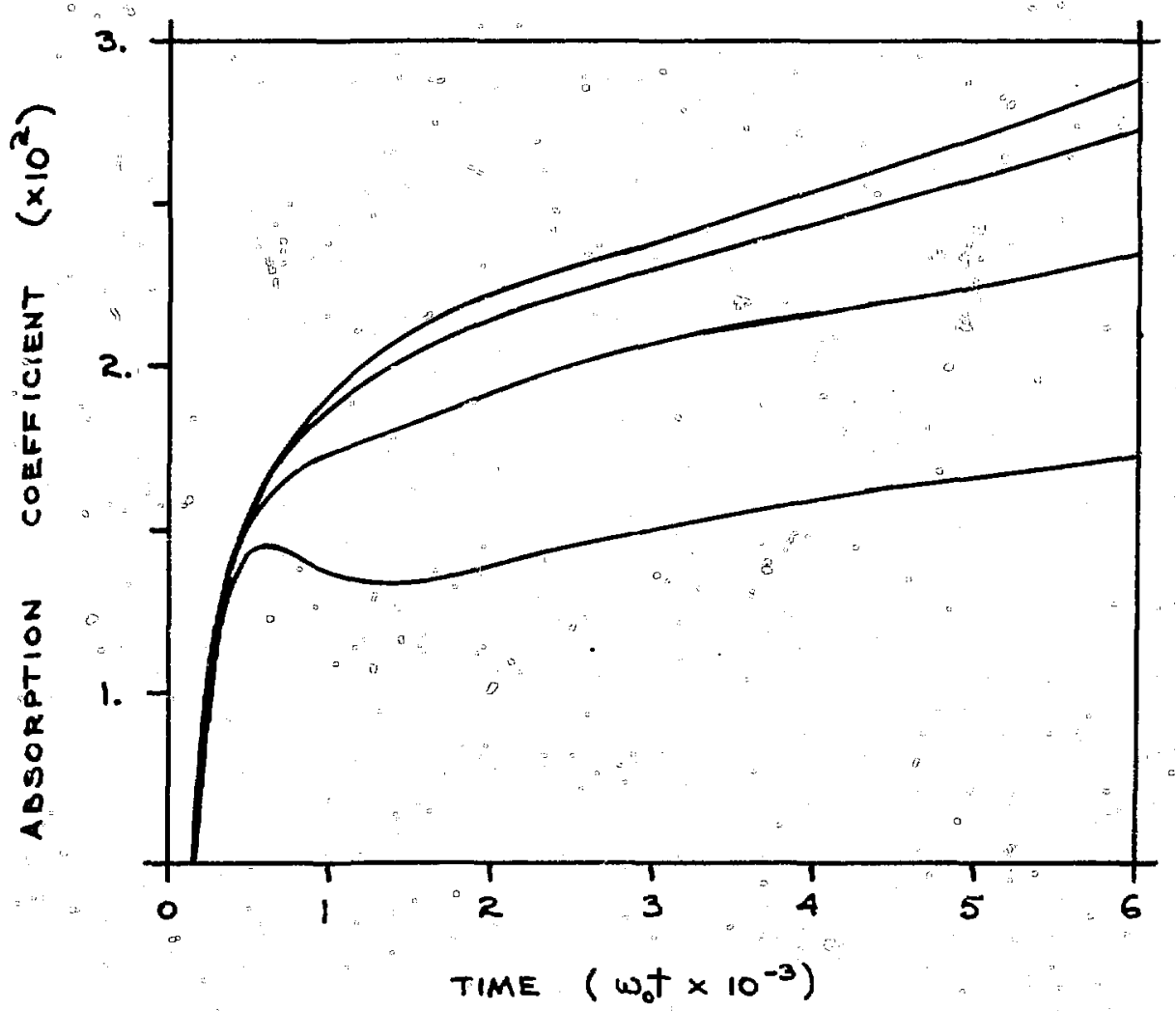

FICLRE :-4 Absorption histories showing the effect of selfsteepening for $=.0025 ; " \mathrm{~m} / \mathrm{M}_{\mathrm{i}}=.01, \theta_{\mathrm{e}}=0.5 \mathrm{KeV}$, $(V / V)=0.0,0.5,097,8.9$. The combination of steep groseteth and relatively large $v /$ i effectively suppressed sBs in these simulations. 
157

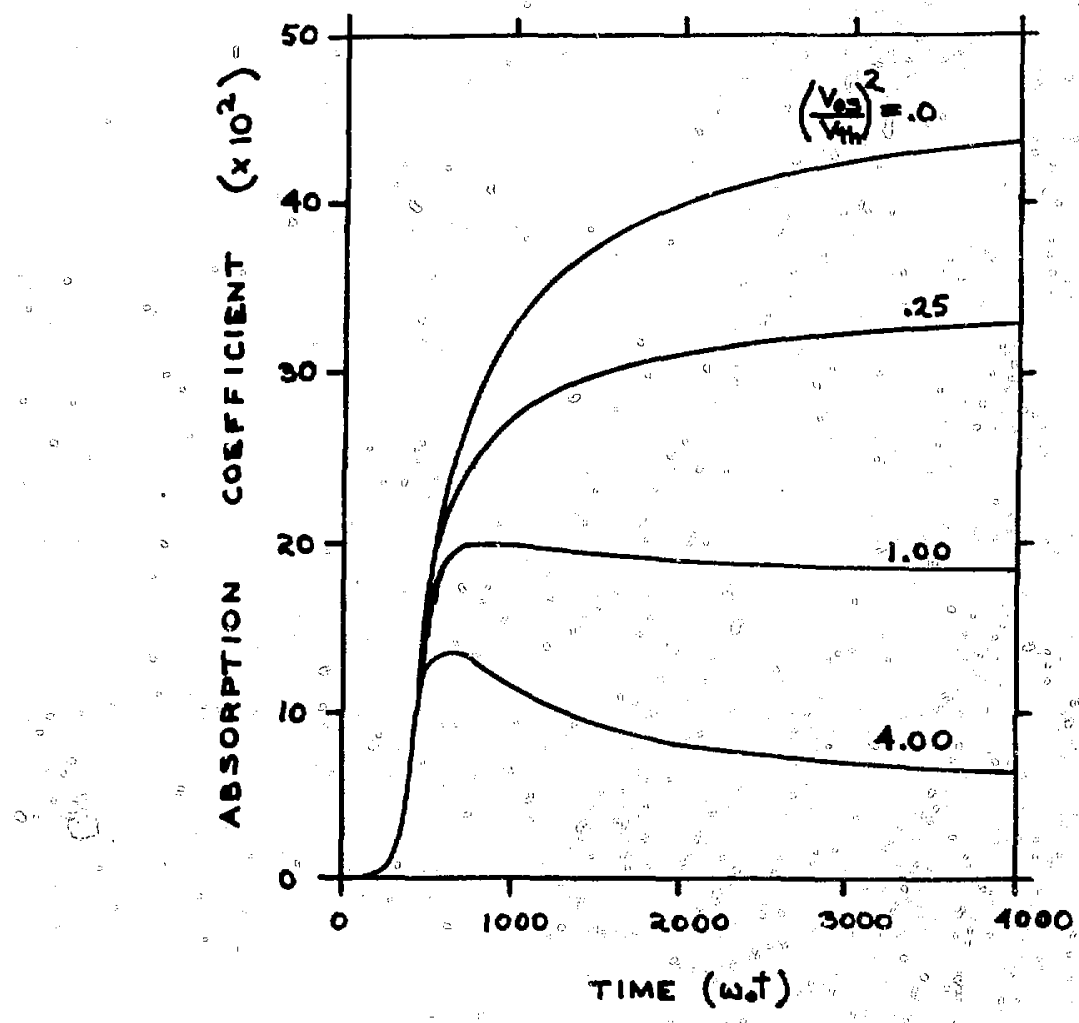

$$
\begin{aligned}
& \text { Figure } 5 \text { Absorption as a function of time }
\end{aligned}
$$

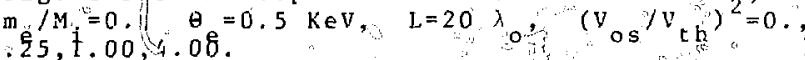




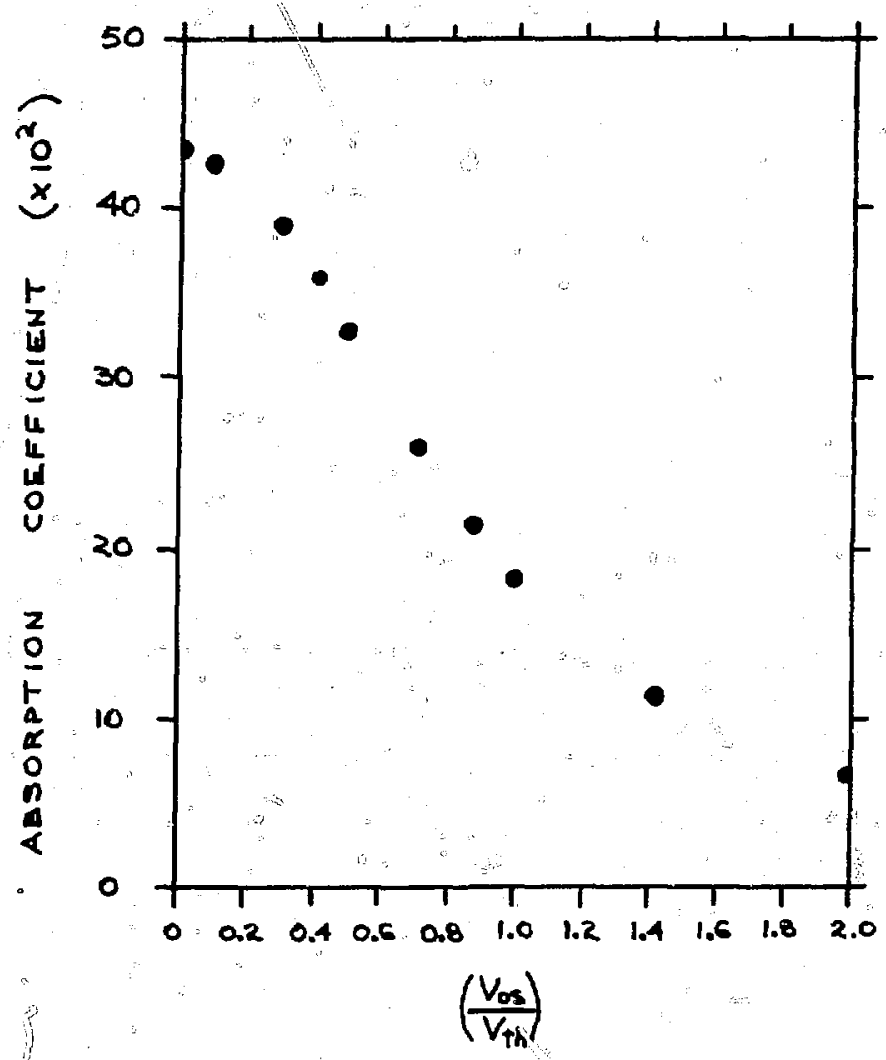

Figure 5-6 Absorption as a tunction of field strength, $m_{e} / M_{i}=0.0, \quad \theta_{e}=0.5 \mathrm{keV}, \quad l=20 \lambda_{0}, \quad z=1.0$. 
139

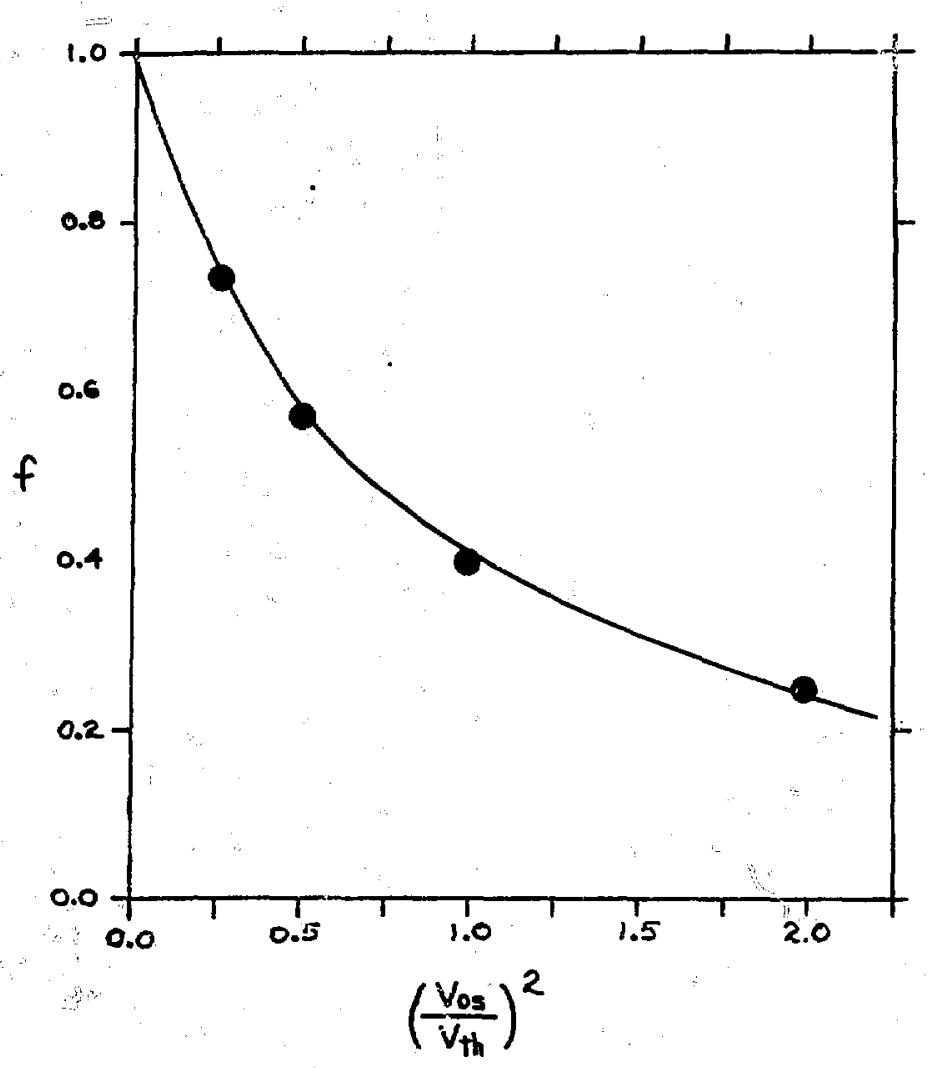

Figure 5-7 Absorption fraction, f, as a function of field strength, $\mathrm{m}_{\mathrm{e}} / \mathrm{M}_{\mathrm{i}}=0.0, \theta=0.5 \mathrm{KeV}, \mathrm{L}=4.8 \lambda$ For purposes of comparison, the solid line is Eq (5-8). 


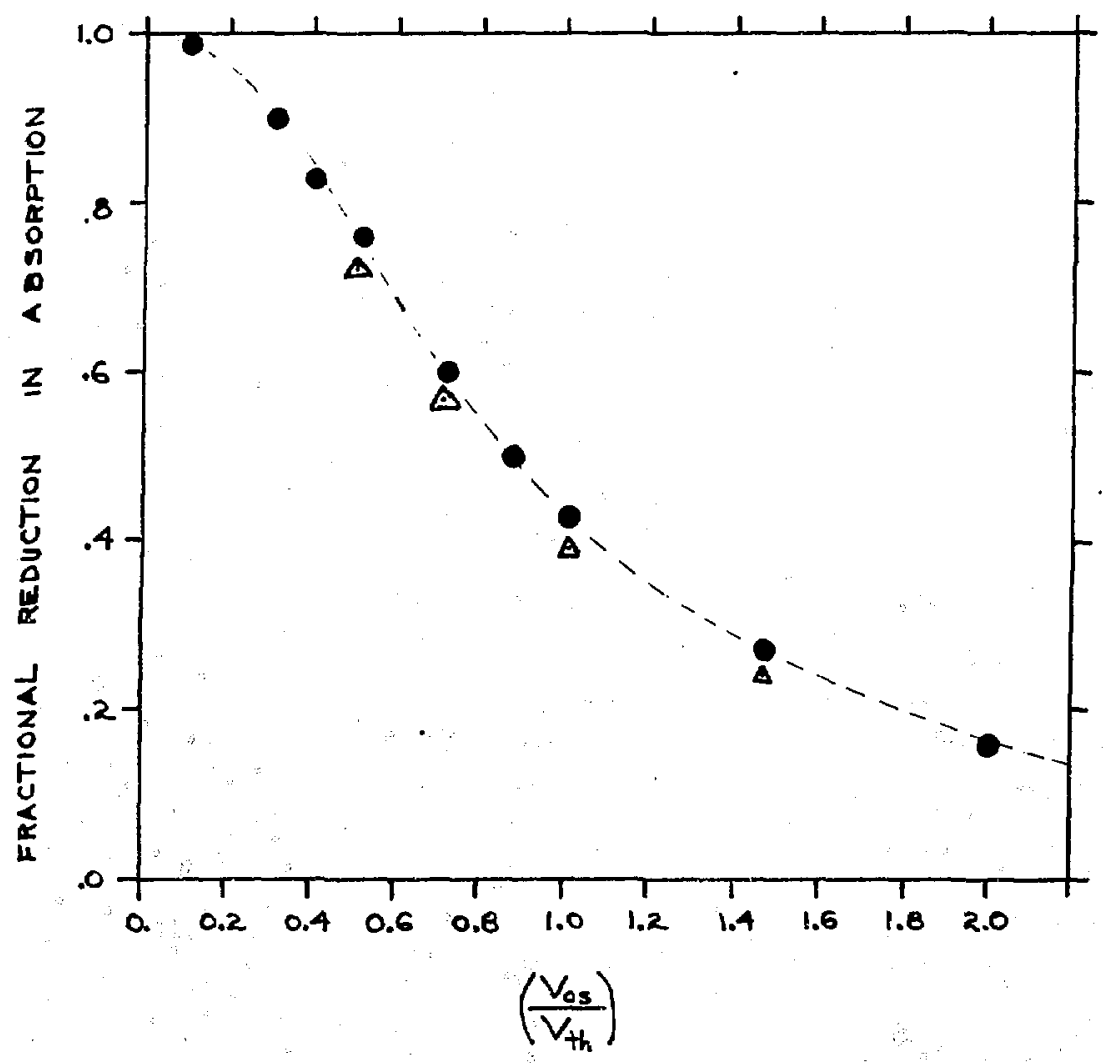

Figure 5-8 Absorption fraction, $f$, as a function of field strength for different scale lengths, $L=21.0 \lambda$ ( ) and

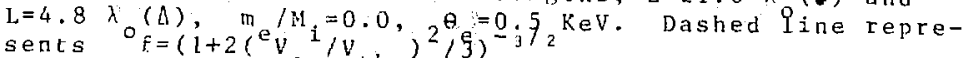




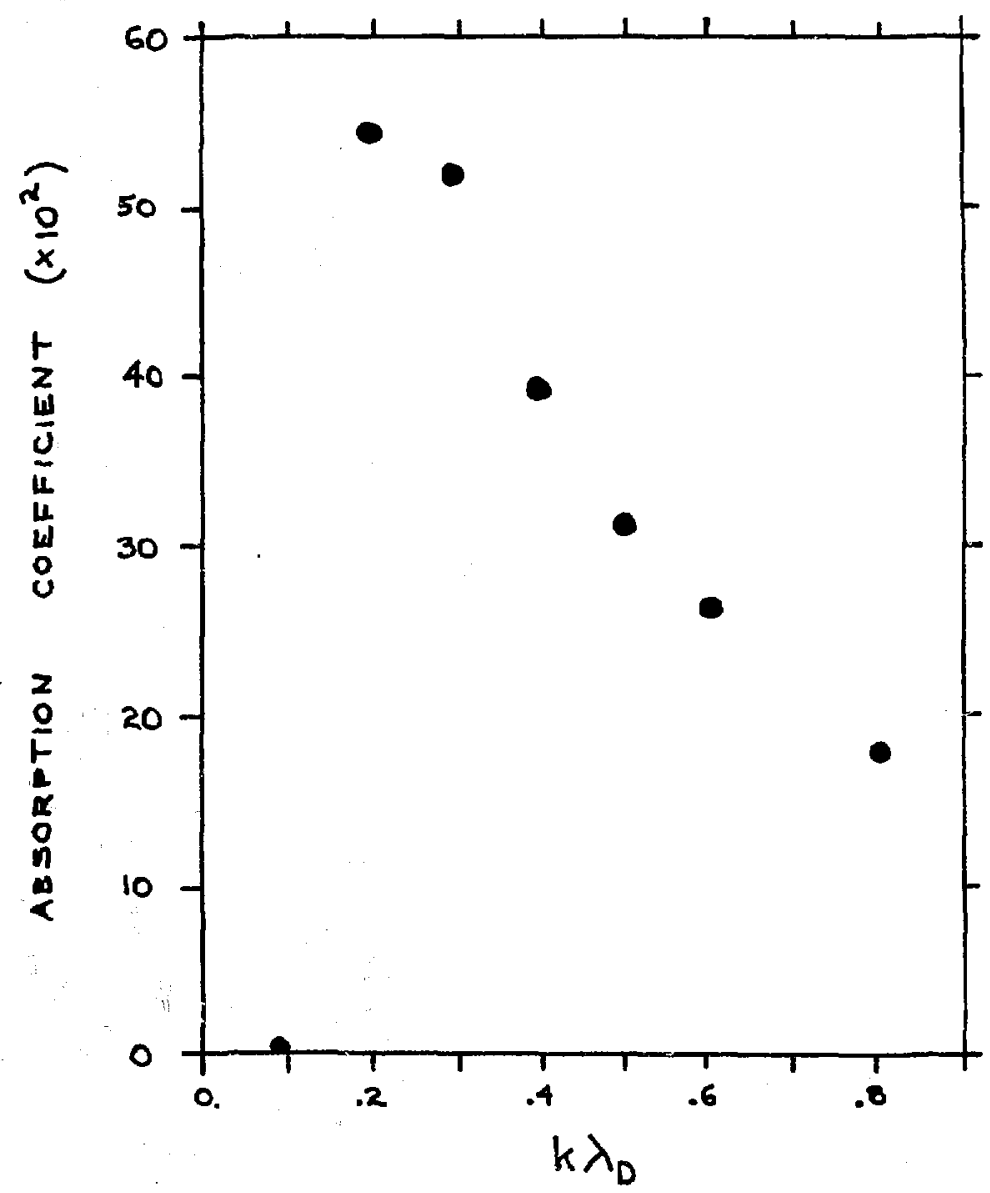

Figure 5-9 Density fluctuation induced absorption as a function of wavelength of the fluctuation, $\Delta \mathrm{n} / \mathrm{n} \mathrm{cr}=.07$, $\mathrm{m}_{\mathrm{e}} / \mathrm{M}_{\mathbf{i}}=0.0, \theta_{\mathrm{e}}=50 . \mathrm{KeV}, \mathrm{L}=21.0 \lambda_{\mathrm{o}}$. 


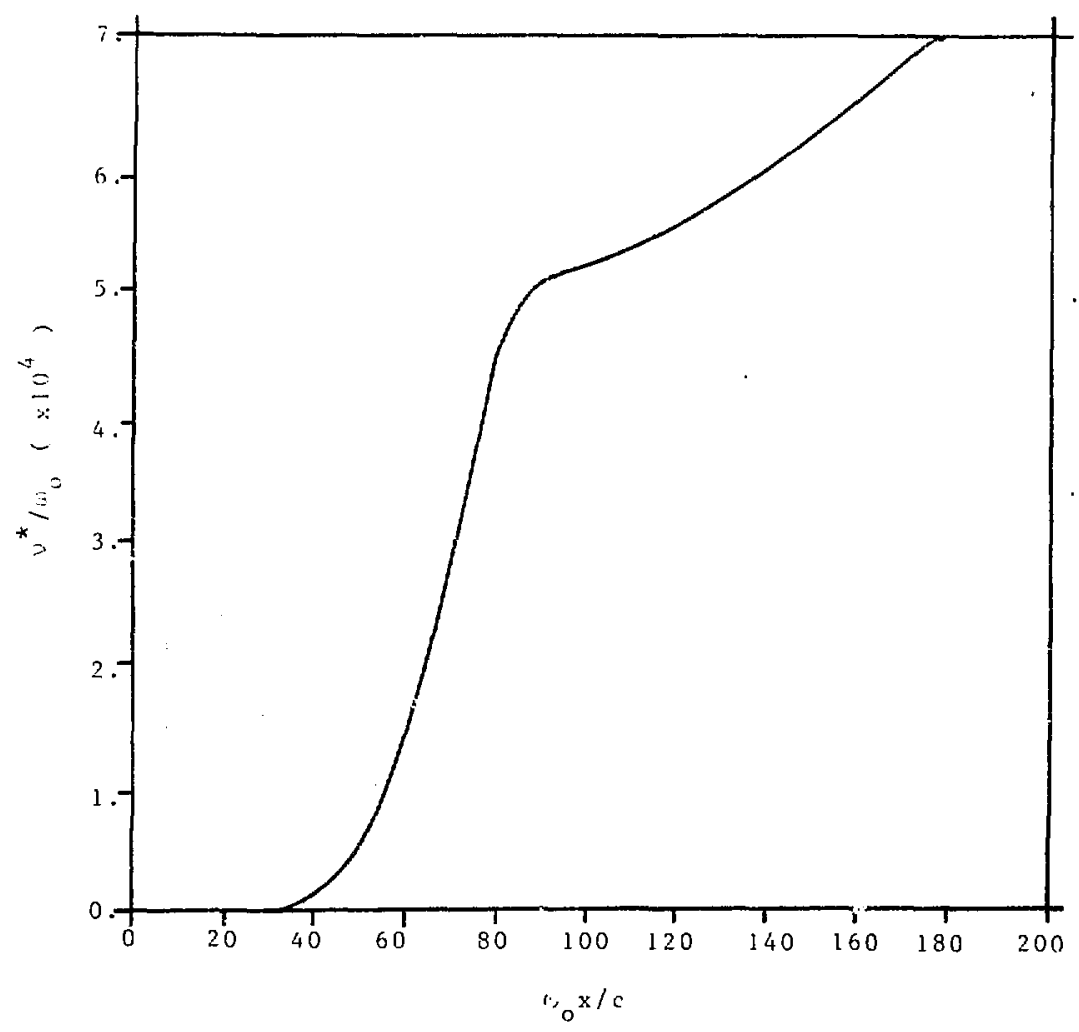

Figure 5-10 Density Eluctuation induced absorption rate, $v^{*}$ as a function of position, same density profile as in Figure 5-11(a), $\mathrm{k}_{\mathrm{D}}=0.5, \quad \Delta \mathrm{k} \lambda_{\mathrm{D}}=0.5, \Delta_{\mathrm{n}} / \mathrm{n}_{\mathrm{Cr}}=.07$. 


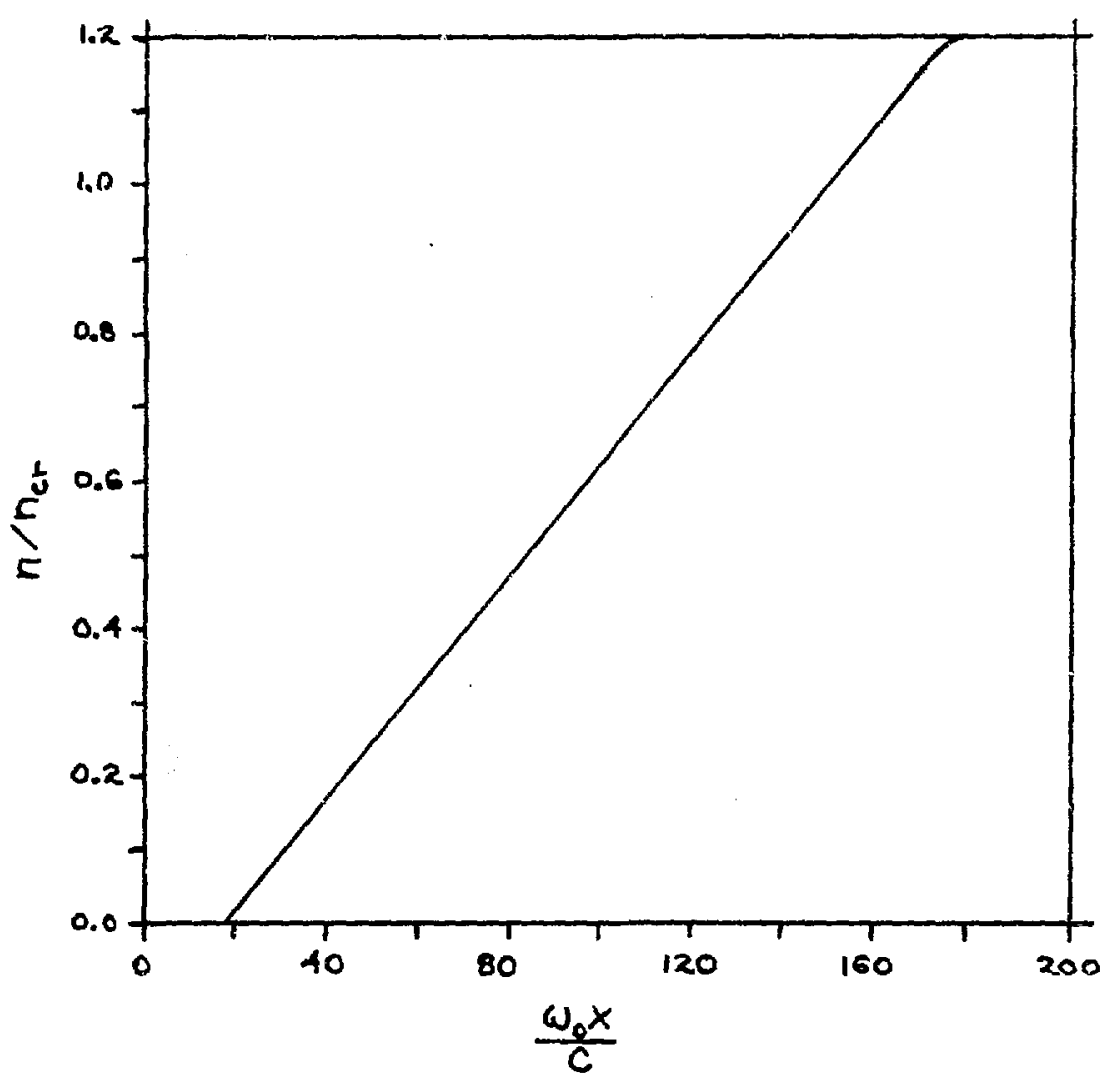

Figure 5-11(a) Density profile used for computing the density fluctuation induced absorption rate, $v^{*}$, in Figures 5-11(b)-(j). Fivctuations are assumed to be uniformiy distributed over the entire profile, $L=21.0 \lambda_{0}$. 


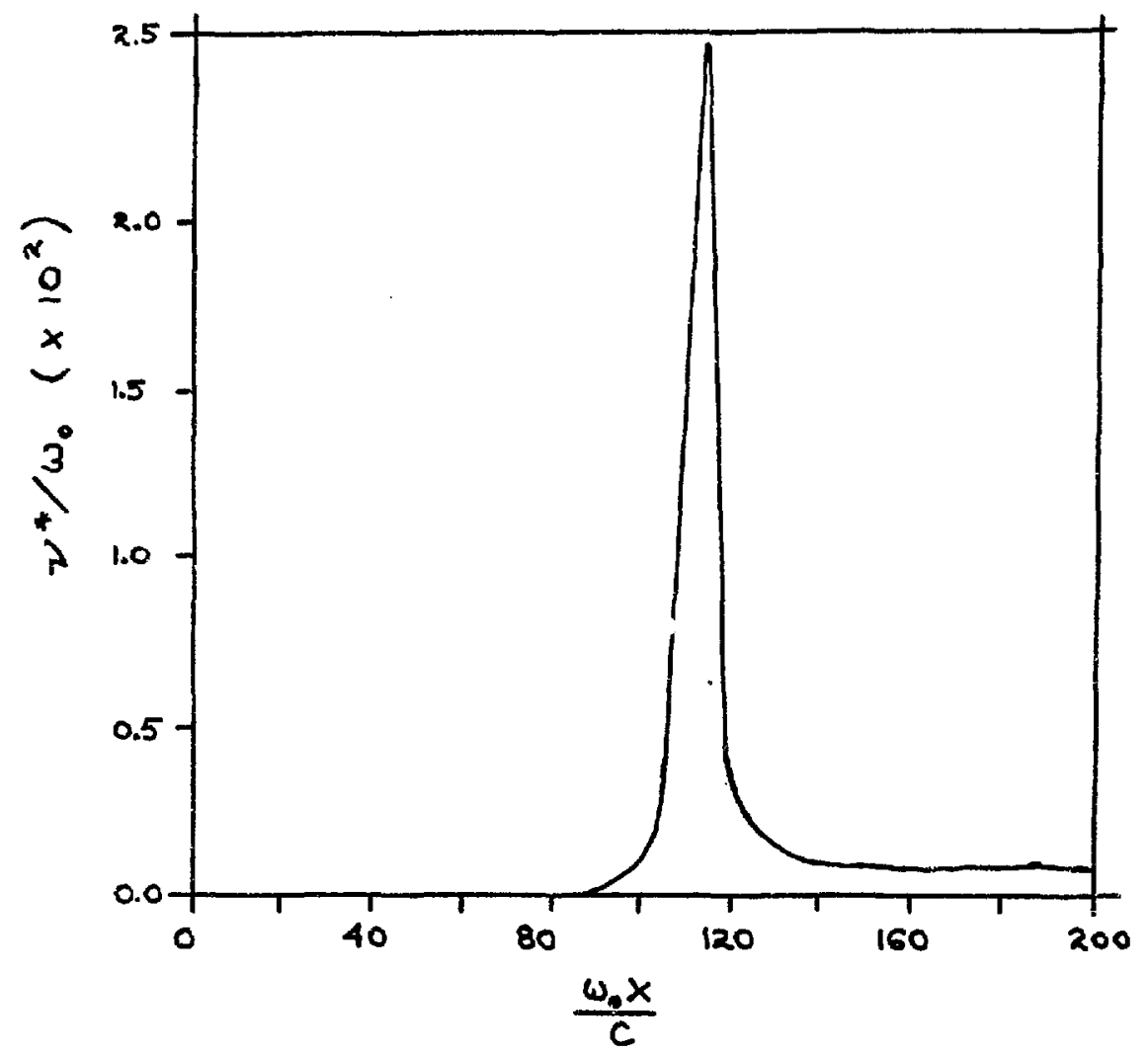

Figure 5-11(b) Density fluctuation induced absorption rate, $v^{\text {* }}$ $\mathrm{k} \lambda_{\mathrm{D}}=0.3, \Delta \mathrm{n} / \mathrm{n}_{\mathrm{cr}}=.0 \%$ 


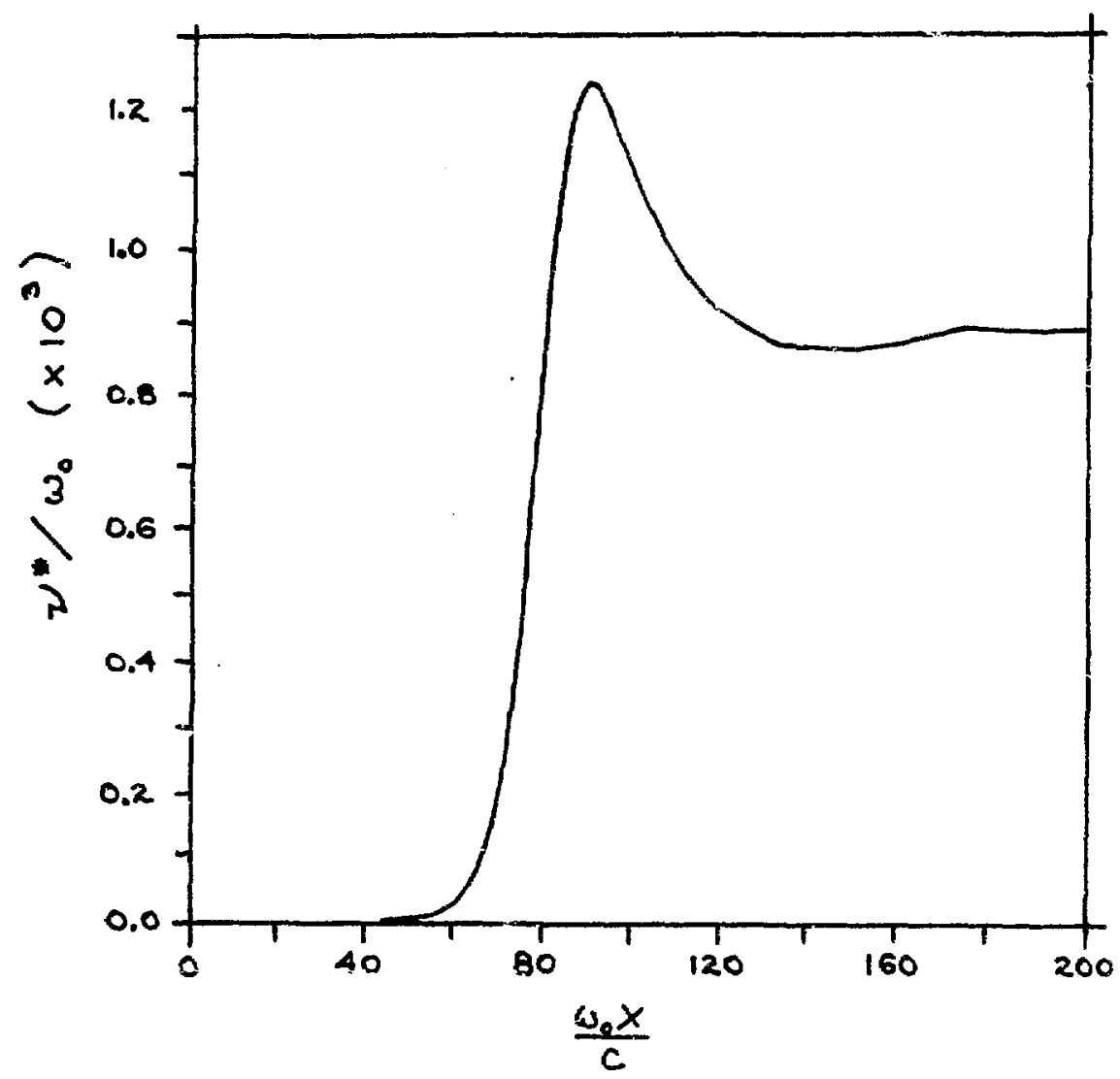

Figure 5-11(c) Density fluctuation induced absorption rate, $k \lambda_{\mathrm{D}}=0.5, \Delta \mathrm{n} / \mathrm{n}_{\mathrm{cr}}=.07$. 
156

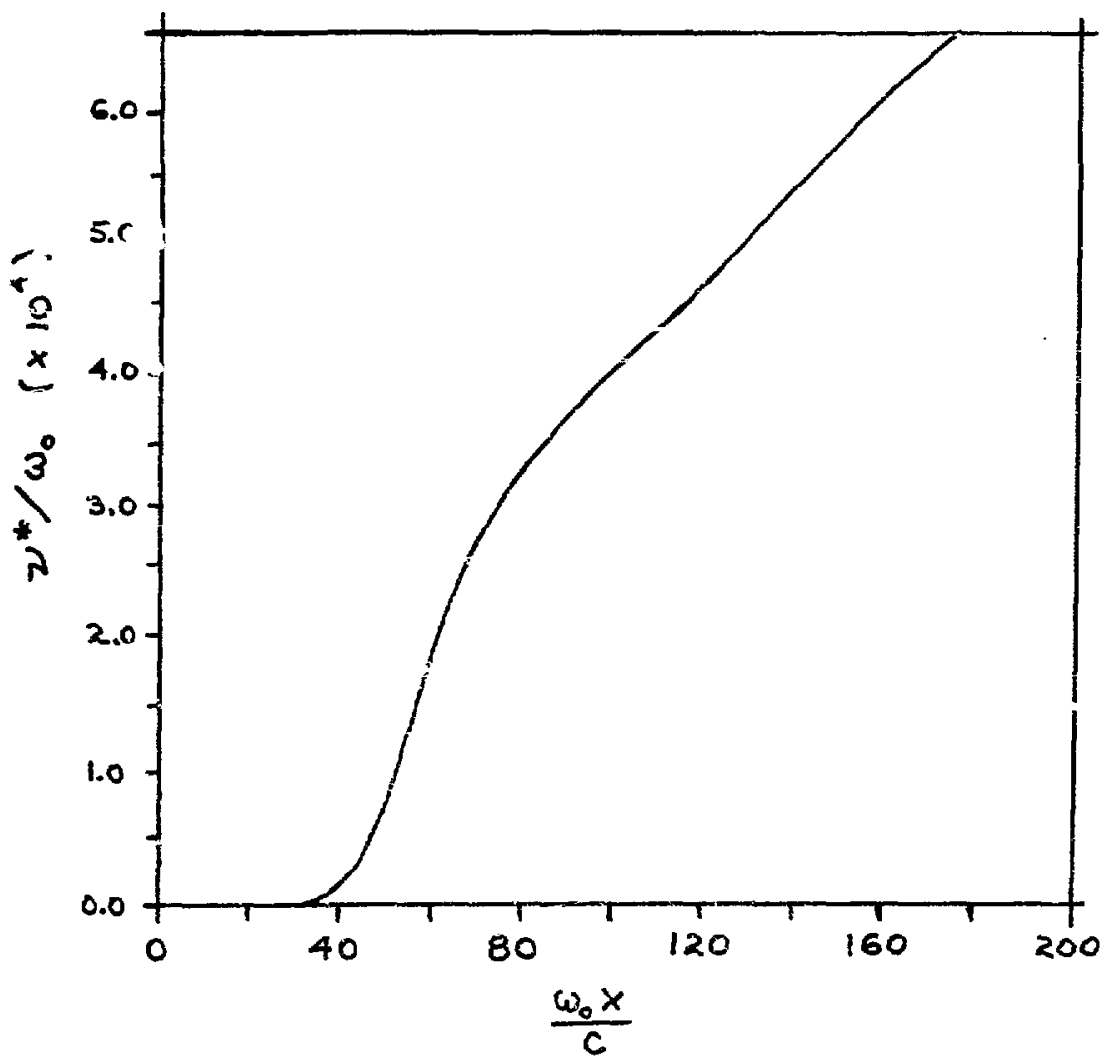

Figure 5-1i(d) Density fluctuation induced absorption rate, $v^{*}$ $k_{\lambda}=0.8, \Delta \mathrm{n}_{\mathrm{D}} / \mathrm{n}_{\mathrm{r} . \mathrm{r}}=.07$. 


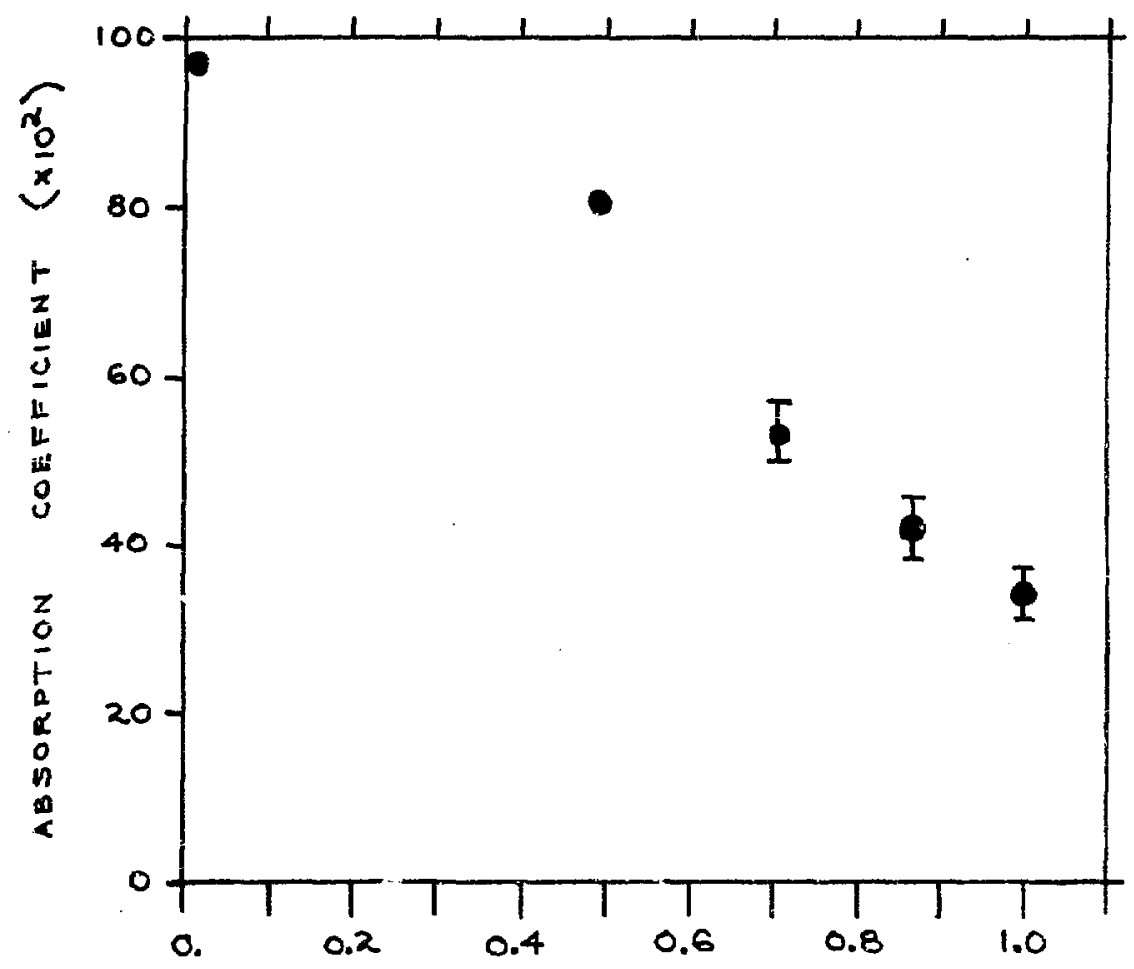

$\left(\frac{V_{0 s}}{V_{t h}}\right)$

Figure 5-12 Absorption as a function of field strength, $\begin{aligned} \theta & =i .0 \mathrm{KeV}, \quad \mathrm{L}=21 \lambda_{0}, \quad \mathrm{k} \lambda_{\mathrm{D}}=0.5, \quad \Delta \mathrm{n} / \mathrm{n}_{\mathrm{cr}}=0.07, \quad \mathrm{~m}_{\mathrm{e}} / \mathrm{M}_{\mathrm{i}}=0.0 \text { ?. } \\ v_{\mathrm{i}} & =0.0025 \mathrm{\omega}_{0} .\end{aligned}$ 


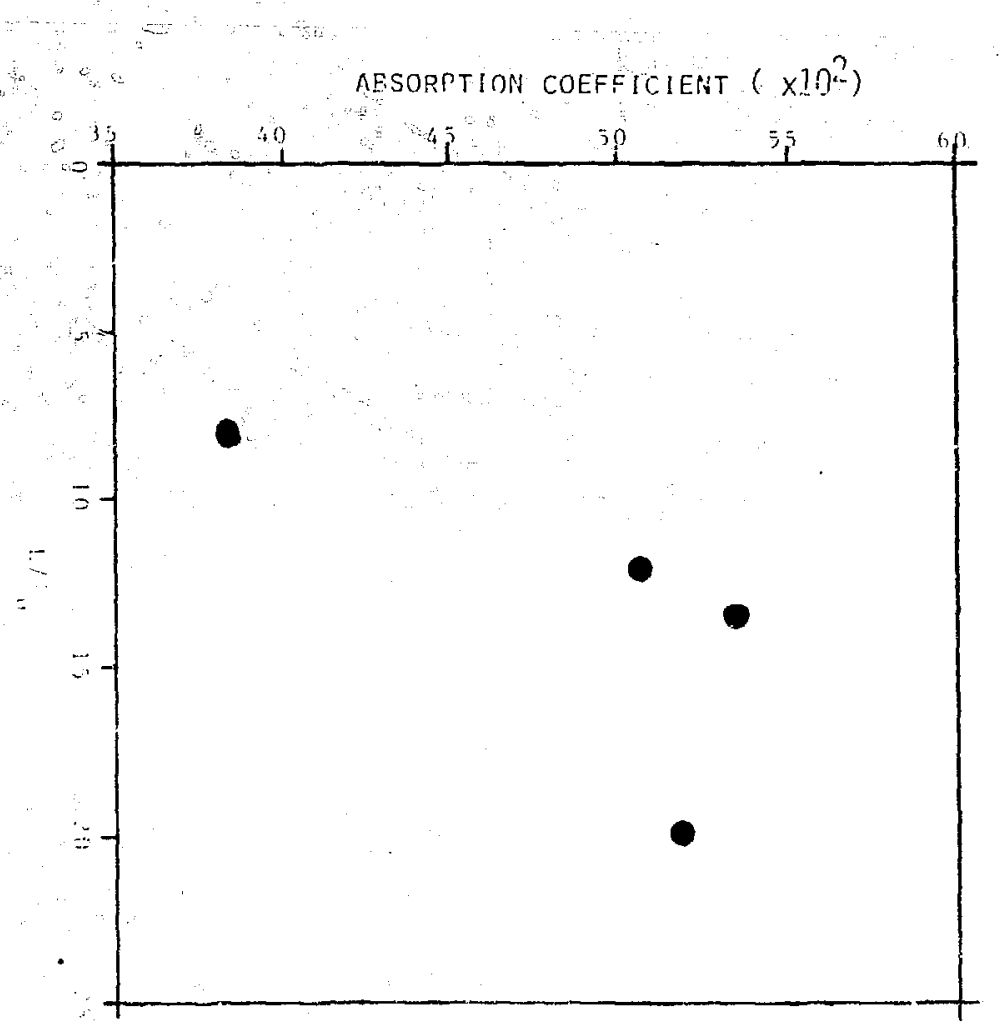




\section{CHAPTER 6}

Conclustons and Recommendations

The numerical method described in this work has been demonstrated to be a last, flextble tool for examinirg a varety of laser plasma problers. The technique of solving the two fluid plasma equations on the plasma time scale, coupled jelf-consistently with Naxwell's equations, has proved useful to studying scatering, modulational, and absorptive phenorina. Ahough the conclusions reached in the course of this work have rotential applications to laser fusion, particularly in the iscas of urderstand ing "bubble" fortatior and self-consistent

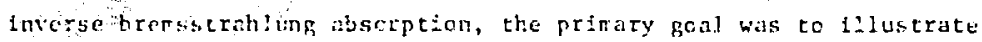

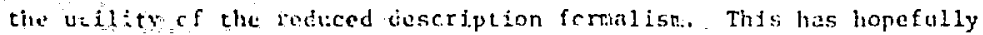
teen aciorip? 1sties.

scueral linitations and flaks in the por cechntque have been lis-

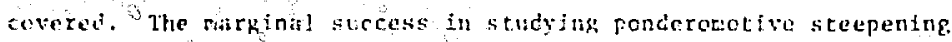
near the orition censity apely showed the difrusiveness of the numet-

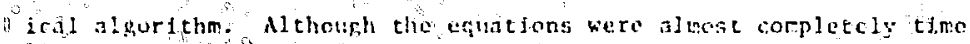

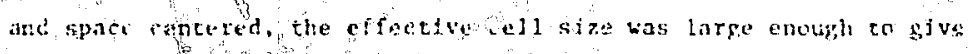
certiderate thestan sroothing. This ray have contributed to the

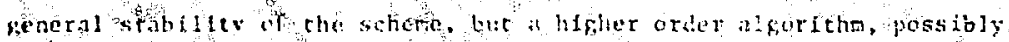

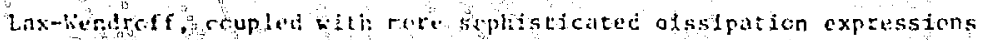

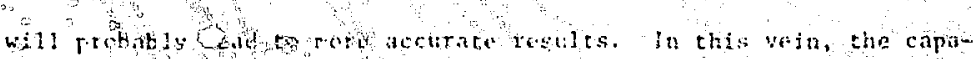

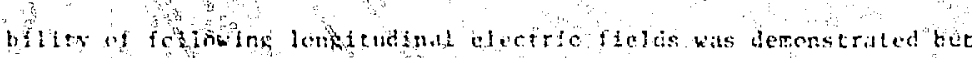

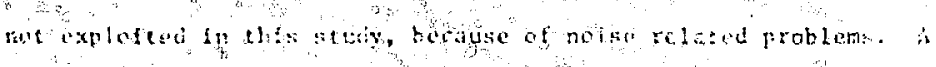


dissipation expressicu tof the electon fludid which more closciy models Landau datping flould open corpletely new classis of protalems for Fur investigation.

The RDT technique can alse be extended hy including, nigher croter woment equations and transport equations appropriate tc other quantities. A temperature equation las been implemented into the schere already, without excessive difficulty. Although the themal comiuction: was usually high enough to justify the isothemal assunption, prob. lems imolving self-consistent heating necd to treat the crerg: balance properly. Asc, dense. high temperature plasnas arc yellknow emitters of high frequency radiation, such as X-rays. This radiation requites a much shovet tine scale than the lisey, and se ic not calculated in tia field equations. Various cochiques for tratsperting suth radiation are knowe, hokever, anc one of these nipto he profitably incorporated 1nte the for equations.

Finally, higler dimensional effects cleirly are iremeant to

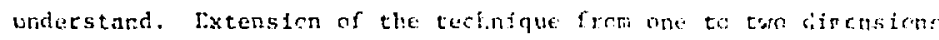

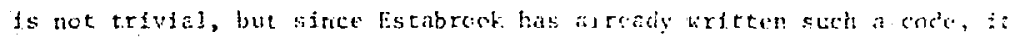
apparenty js feasible. Nhthugh such a numerical toc bould bo bo at

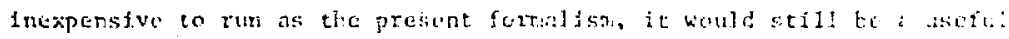

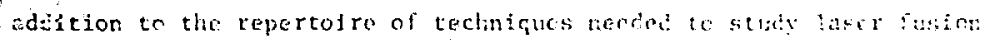
problens.

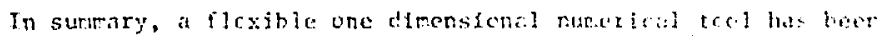

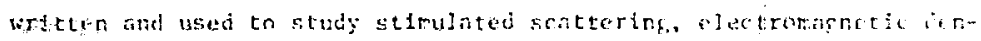
sity profilo modulation, selr-consistert andse hachetrahums

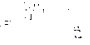


abscrption, shoth/ wavelength ion density induced absorption, steepening near the critical denstey, and elactronagnetic field structure of Tr. waves near the critical density. These were studfed nerely ats representacive types of proh!eme for which the techntaue can be useful. "Fxtensions of the code have furthermere been suggested wicli now Increase the number of applicat/fons even more. The litility of the technique, and fos potentfil for future use, have thus been aris!y ciefoustorted. y

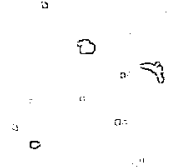
8 
APPENDIX A

The Reduced Description Equations

Derivation of the reduced destription fo: lism is : . aight forward. The complete description is the livalilfo equation Wor the N-particle distribution function,

$$
\frac{d f^{N}\left(\bar{x}_{N}, \bar{v}_{N}, t\right)}{d t}=0
$$

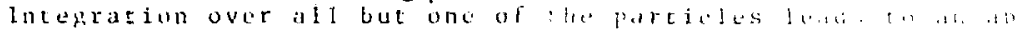
propriate ono particle distribution,

$$
\frac{\partial f}{\partial t}+\nabla \cdot \frac{\partial f}{\partial \bar{x}}+\frac{\bar{E}}{m} \cdot \frac{\partial f}{\partial \bar{v}}=\left(\frac{\partial f}{\partial t}\right)_{c_{0} \| l}
$$

where $f=i(\bar{x}, \vec{v}, t), \vec{F}$ is the force at the phase spat

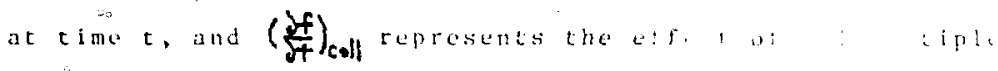
particle interactions on $E$. This level of desctipt, th be

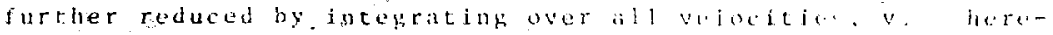

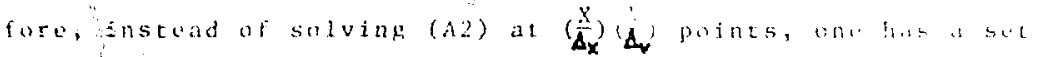

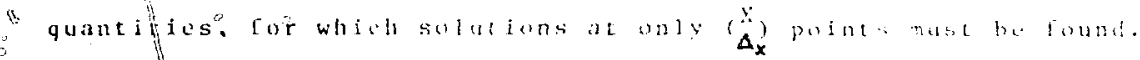
The quaptities are formed by takink vele ity monents at lal.

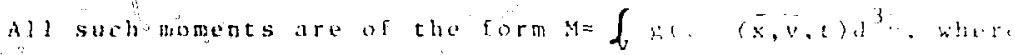
$g=1$ avis $y=n(x, t)$, the nuber density, g=v pives $\because$ nu, the

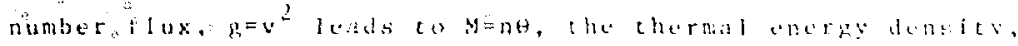
and so, forzli, Explicity,

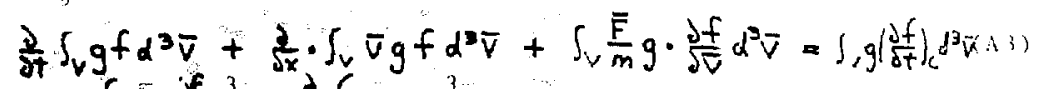

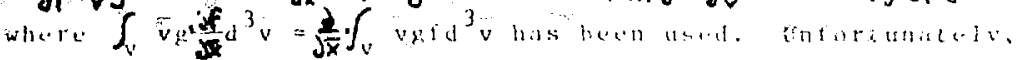

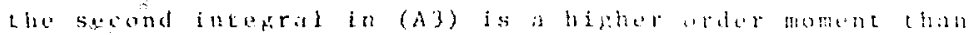

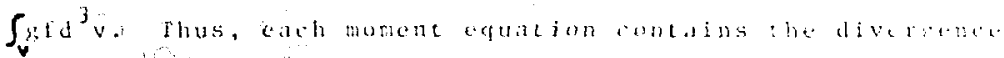

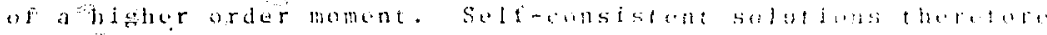

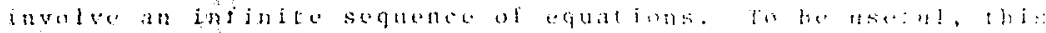

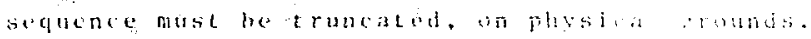


The reduced description employed here consisted of the iirst two moment enations for ions and the first two for a lectrons. The first moment, $y=1$, is just the continitey "quation, (specie notation has been suppressed)

$$
\frac{\partial n}{\partial t}=-\frac{\partial}{\partial \bar{x}} \cdot n \bar{u}+S
$$

where S indicates wiber a source or sink for the specie, i.e. balzation ur recombinatien. Usually, we took $s=0$.

The siond mement equation, $\xi=\bar{v}, i s$ a somentum transfer anuation,

$$
\frac{\partial n\langle\bar{v}\rangle}{\partial t}+\frac{\partial}{\partial \bar{x}} \cdot n\langle\bar{v} \bar{v}\rangle=-\frac{n}{m}\langle\bar{F}\rangle+\left\langle\left.\bar{v} \frac{\partial f}{\partial t}\right|_{\text {coll }}\right\rangle
$$

li we separate $i=u+v$, where $u=\langle y\rangle$ and $\langle\vec{\nabla}\rangle=0$. the divergence. Lerm suparaces inte a convective and a prossure expression

$$
\frac{\partial n \bar{u}}{\partial t}=-\frac{\partial}{\partial \bar{x}} \cdot n \bar{u} \bar{u}-\frac{1}{m} \frac{\partial}{\delta \bar{x}} \cdot \vec{p}-\frac{n}{m}\langle\bar{F}\rangle+\left\langle\bar{v} \frac{\partial f}{\partial t} k_{\text {coll }}\right\rangle(\lambda \sigma)
$$

The averased tarac is derived from Maxwel I's equations It

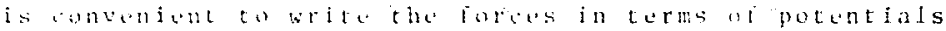

Hbicrution that

$$
\langle\bar{F}\rangle=q\left(-\frac{\partial \varphi}{\partial \bar{x}}-\frac{1}{c} \frac{\partial \bar{A}}{\partial t}+\frac{\bar{u}}{c} \times\left(\frac{\partial}{\partial \bar{x}} \times \bar{l}\right)\right)
$$

a...

$$
\bar{u}_{\perp} \doteq-\frac{e \bar{A}_{1}}{m_{1} c}
$$

$$
\left\langle F_{1}\right\rangle=-q \frac{\partial \varphi}{\partial x}+\frac{1}{2} \frac{q e}{m_{e} c^{2}} \frac{\partial A^{2}}{\partial x}
$$

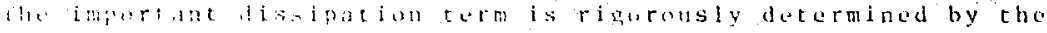

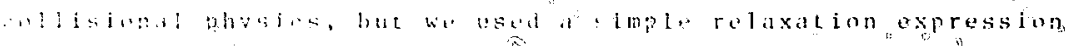

$$
\left.\left\langle\left. v \frac{\partial f}{c t}\right|_{\text {coll }}\right\rangle=-2\right\rangle \bar{u}
$$

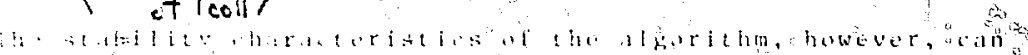

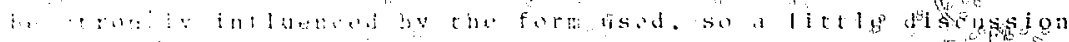

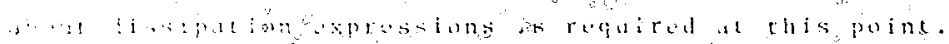


The effect of a constant relaxation rate on an equation like (A6) can be analyzed simply. First, it is clear that flux-perturbations are exponentially damped

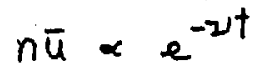

operating: on $\left(\Lambda_{-}\right)$wish $\frac{\partial}{7}$ and on $(A 6)$ with $\frac{\partial}{\partial x}$ leats to

$$
\text { Jn } \propto-2 n
$$

!n other words, all density fluctuations are dameced at

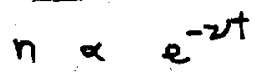

This is probably adequate for ion wayes, since nuther ion Landau damping nor ion collisions exhibit strong wavelength dependence: Eiectron fluctuations, though, exhibit a pronounced damping at short waveleagths. Eq (AB) is, thereiore, inadequate for electron fluids. Marginal stability was achieved

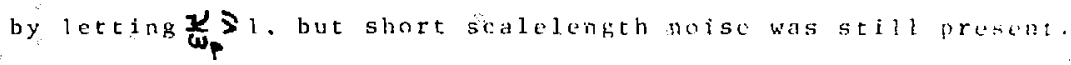
For electrons, two alternative forms may lead to imprusment. viscosity is a well understood fluid dissipation mechanism. "By analogy with viscosity. it is plausible to take

$$
=\frac{\partial n \bar{u}}{\partial t}, \alpha \cdot D \frac{\partial^{2}}{\partial x^{2}} n \bar{u}
$$

This also" leads to

$$
\frac{\partial n}{\partial t} \propto D \frac{\partial^{2}}{\partial x^{2}} n
$$

Thus this type of damping yields a diffusivi stacisting on both flux, nu, and on densitv rluctuations. It has the tesisable property that short wavelengehs aro more damped than long wavelengt ones

$$
n \propto e^{-k^{2} D t}
$$

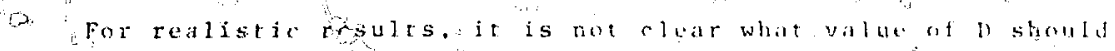
used. Tha diffusion coeffirient must therofore be chosug on 
be used. The diltusion coeflicient must therefore be chosen an physioal grounds to give a desired damping magnitude at a proticular wavelongth.

A veond romonly used form for dissipating gradielits in

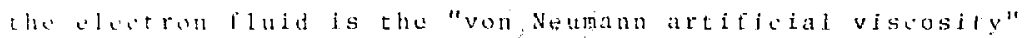

\section{$\frac{\partial n \bar{u}}{\partial t} \propto Q \frac{\partial u^{2}}{\partial x}$}

whore ! is:a sonstant ol order unity. This form is difficult ta and ya besuse bf its intrinsic nonlinoarity. It has proved hishly sutcosstul, thowever, in the salditation of shock phenomena. wor treating waves, (Ali) is not as useful as (AlO). other axpression besides these two could doubtless be dovised, but thege examples wete fole te be jllustrative of the problens

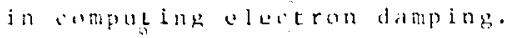

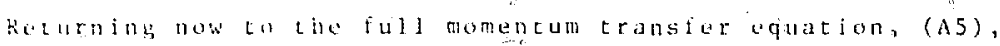

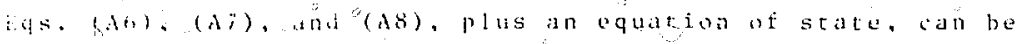

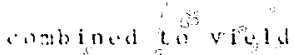

\section{$\frac{\partial n \bar{u}}{\partial T}=-\frac{\partial}{\partial \bar{x}} \cdot h \bar{u} \bar{u}-\frac{\theta}{m} \frac{\partial n}{\partial \bar{x}}+\frac{q n}{m} \frac{\partial \phi}{\partial x}-\frac{1}{2} \frac{q e n}{m c^{2}} \frac{\partial A^{2}}{\partial \bar{x}}-\nu n \bar{u}(A 12)$}

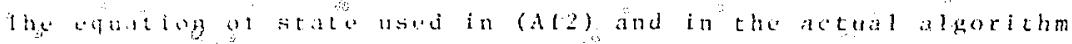

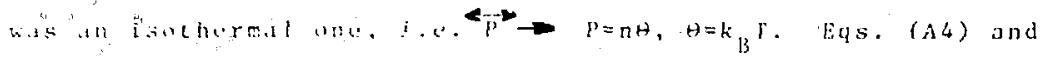

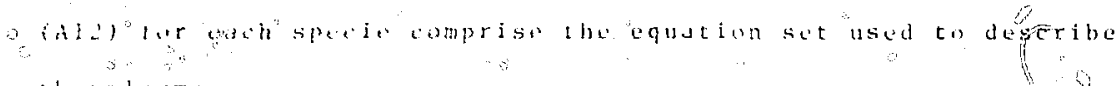
i he platint.

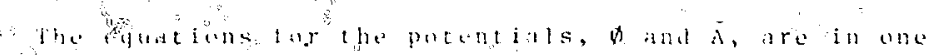

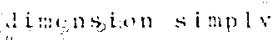

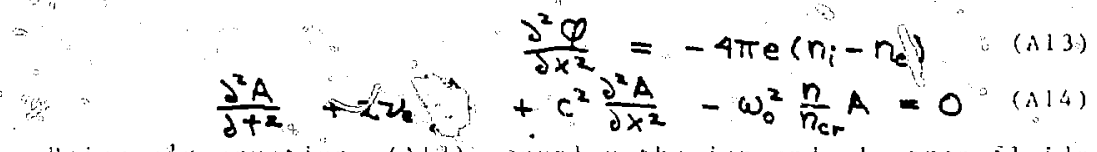

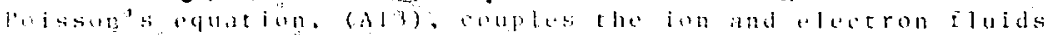

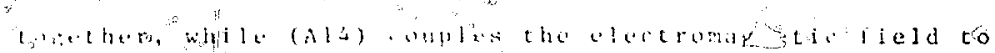


Logether, while $(A ! 4)$ couples the electromagnelic field to the electron fluid. Note that the gauge condition for (Al3) and $(A(4), \nabla \cdot \bar{A}=0$, is triviliy satisfied in slab geometry.

Finally, the number of equations could be even further reduced by assuming the the electrons were inertiaiess, lliat is $\frac{d \bar{u}}{d t}=0$. Then i.t follows that

or

$$
0=-\frac{\theta_{e}}{m_{e}} \frac{\partial n}{\partial x}-\frac{e n}{m_{e}} \frac{\partial P}{\partial x}-\frac{1}{2}\left(\frac{e}{m_{c}}\right)^{2} n \frac{\partial A^{2}}{\partial x^{2}}
$$

$$
\operatorname{en} \frac{\partial \varphi}{\partial x}=-\theta_{0} \frac{\partial n}{\partial x}-\frac{1}{2} \frac{e^{2}}{m c^{2}} n \frac{\partial^{2} A^{2}}{\partial x}
$$

(Al5) can then te substituted into the ion momentum equation

$$
\frac{\partial(n u)_{i}}{\partial t}=-\frac{\partial}{\partial x} \frac{(n u)_{i}^{2}}{n_{i}}-\frac{\theta_{c}+\theta_{i}}{m_{i}} \frac{\partial n_{i}}{\partial x}-\frac{m_{1} n_{i}}{m_{i}}\left(\frac{e}{n_{c} c}\right)^{2} \frac{\partial A^{2}}{\partial x}-\nu(n u)_{i}(A 16)
$$

where we have assumed that the electron and ion gradiertis are virtually identical. Now we proceed to the differencing of the equations, (A4), (A14), and (Al6).

For convenience, the quanticies are normalized so that $c=1, \omega_{0}=1$, and $n_{c r}=1$. The vector potential is normalized to its vacuum alignitude, $A_{0}$, and the field strength paraneter $v_{o s} / v_{t h}=e A_{0} /\left(m c^{2} \theta_{e}\right)^{\frac{1}{2}}$, is represented as $Y$ below. Also let $s_{1}=\frac{\Delta t}{\Delta x}, s_{2}=\frac{\Delta t}{(\Delta x)^{2}} \cdot s_{n}=(1+.5 \nu \Delta t), s_{4}=(1-.5 \nu \Delta t)$, and $s_{5}$ and $s_{6}$ like $s_{3}$ and $s_{4}$ except that $\boldsymbol{Z}_{\mathrm{e}}$ appears, in place of $\boldsymbol{Z}$.

$$
\begin{aligned}
& n_{j}^{i}=n_{j}^{i-1}-.55_{j}\left(g_{j+1}^{i-k_{2}}-g_{j-1}^{i-v_{2}}\right)
\end{aligned}
$$

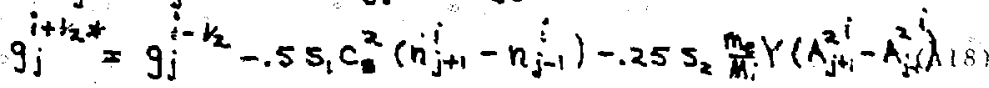

$$
\begin{aligned}
& g_{j}^{i+V_{2}}=g_{j}^{i+s_{2}^{*}}-5 s_{1}\left(\left[g_{j+1}^{i+k^{*}}\right]^{2} / n_{j+1}^{i}-\left[g_{j-1}^{i+k_{2}^{*}}\right] / n_{j-1}^{i}\right) \\
& \dot{A}_{j}^{i+K_{2}}=A_{j}^{i-V_{2}} \frac{s_{s}}{S_{s}}-\frac{\Delta t}{s_{s}} n_{j}^{i} A_{j}^{i}+\frac{B_{2}}{S_{s}}\left(A_{j-1}+A_{j-1}^{i}-2 A_{j}^{i}\right)(A n)
\end{aligned}
$$




\section{APPENDIX B}

Linear Theory of the Stimulated Brillouin/Zero-Frequercy
Instability

This appendix s devoted to a derivation and analysis of the linear theory of the instabilitles discussed in Chapters 3 and 4. The derivation is not in itself originai, and in fact follows the formalism of Nishikawa $54, i$ e. the instability of two coupled harmonic oscillators driven by a large amplitude pump wave. To the reader familiar with Ref. 54, the rotation may lead to confusion. The symbols used are similar enough to provoke recognition, but unfortunately they do nut al ways have the same connotations. The high frequency waves, For instance, are denoted as waye 1 , and the low frequency as wave 2. This is precisely opposite to the notation used by Nishikawa. The reader will hopefully kep this in mind while going through this section. An apology is perhaps also due for the detal included here. Without going through every step, it is hoped that the unfamiliar reader wlll be able to follow the development more easily because of this strategem. orhese instabilities inuolve the coupling of an electromagnetic wave with a secontary electromagnetic wave and an ion density fluctuation. The wave equation for the electromagnetic wave is dírectlyobtainable from Maxwell's equations oritten in terms of potentials, in the transverse gauge, $\nabla \cdot \bar{A}=0$. The equation for $\vec{A}$ i.s the only one needed in this case;

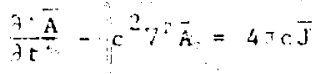

The source torm, $\vec{l}$, in the absence of transverse gradients can 
be accurately expressed as $J=n_{0} \mathrm{e}^{2} \mathrm{~A} / \mathrm{m}_{\mathrm{e}} \mathrm{c}$ in a homogeneous, nonrelativistic plasma. (vector notation will henceforth be suppressed.) The by Fourier analyaing (B 1), one obtains the Fol Lowing linearized equation

$$
\frac{\partial^{2} A_{k_{1}}}{\partial t^{2}}+\left(\omega_{p}^{2}+k_{1}^{2} c^{2}\right) \dot{A}_{k_{1}}+2 \Gamma \frac{\partial A_{1}}{\partial \tau} k_{1}=-\frac{\omega}{n}_{0}^{2} n_{k}^{*}-k_{0} A_{k_{0}}
$$

where $\omega_{p}^{2}=4 \pi e^{2} n_{0} / m_{e}$ is the local plasma frequency. We have taken the instantaneous density to be of the form $n(\bar{r})=n_{0}+n(\bar{r})$, and added a phenomenological damping term proportional to $2 \Gamma$.

The equation for the density fluctuation is deirived from the RDF equations in Appendix $C$, and so is not duplicated here. After Fourier analyzing the ion wave equation, the following results

$$
\frac{\partial^{2} n}{\partial t} \frac{n}{z} k_{2}+\frac{k T}{M_{i}} e k_{2}^{2} n_{k_{2}}+2 \Gamma \frac{\partial}{\partial} \frac{n_{T}}{\partial} k_{2}=-\frac{1}{2}\left(e / m_{e} c\right)^{2}\left(m_{e} / M_{i}\right) k_{2}^{2} A_{k_{2}}^{*}-k_{o} A_{0}
$$

Equations (B2) and (B3) describe the coupled harmonic oscillators.

In Nishikawa's analysis, a standing wave pump was explicitly assumed

$$
A_{k}\left(\omega_{0}\right)=2 A_{k_{0}} \cos \left(\omega_{0} t\right) \text {. }
$$

This is somewhat less general than it need be, nowever. We will instead assume

$$
\begin{array}{r}
A_{k_{0}}\left(w_{0}\right)=\xi_{2} A_{0}\left\{e^{i k_{0} x-i \omega_{0} t}+e^{-i k_{0} x+i \omega_{0} t}+\alpha e^{i k_{0} x+i \omega_{0} t}+\right. \\
\alpha e^{-i k_{0} x-i \omega_{0} t}
\end{array}
$$

For $\alpha=1$. the pump reduces to a standing wave form, while for $x=0$. a traveling wave pumpesults. The ratingale for this form is that in a laser fusion plasma. the incoming laser signai will propagate into the critical density and be reflected. The fraction of incident intensity that arrives at a given 
point will depend on the amount of dissipation which has occurred at higher densities. It can always be expressed as a mixture of traveling and standing waves, sucin that $0 \leq \alpha \leq 1$.

We now assume that the waves have the temporal dependence $A_{k} v^{i \omega_{1} t}$ and $n_{k_{2}} v^{i \omega_{2} t}$. Eqs (B2) and (B3) then becorie

$$
\begin{aligned}
& \left(-\omega_{1}^{2}-2 i \omega_{1} \Gamma_{1}+\omega^{2} k_{1}\right) A_{k_{1}}\left(\omega_{1}\right)=-\frac{\omega^{2}}{\mathrm{n}_{0}}\left(A _ { k _ { 0 } } \left(\omega_{0} j n_{k_{1}}^{*} k_{0}\left(\omega_{1}-\omega_{0}\right)\right.\right. \\
& \left.+\alpha A_{k_{0}}\left(-\omega_{c}\right) n_{k_{1}-k_{0}}\left(\omega_{1}+w_{0}\right)\right\} \\
& \left(-\omega_{2}^{2}-2 i \omega_{2} \Gamma_{2}+\omega^{2} k_{2}\right) n_{k}\left(\omega_{2}\right)=-\frac{1}{2} n_{0}\left(\frac { e ^ { 0 } } { m e } f ( \frac { m } { M } e ) k _ { 2 } ^ { 2 } \left\{A_{k}{ }_{i}\left(\omega_{0}\right) A_{k_{2}}^{*}-k_{o}\left(\omega_{2}-\omega_{o}\right)\right.\right. \\
& \left.+\alpha A_{k_{0}}\left(-\omega_{0}\right) A_{k_{2}-k_{0}}^{*^{0}}\left(\omega_{2}+\omega_{0}\right)\right\}
\end{aligned}
$$

On the right hand side of (p5a), the frequency dependence must be near $\omega_{k_{2}}$ if resonance with tive normal mode is to be attained. This implies two separate matcting condicions

$$
\begin{aligned}
& w_{0}=-\omega_{1}+w_{2} \\
& w_{0}=\omega_{1}+\omega_{2}
\end{aligned}
$$

The dispersion relation is obtained from (B5b) and the two eguations arising from (B5a)

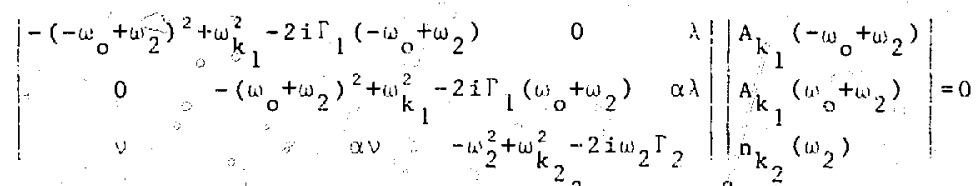

where $\lambda={ }^{2} \mathrm{~A}_{\mathrm{k}} / \mathrm{n}_{\mathrm{o}}$ and $\nu=\operatorname{lin}_{\mathrm{o}}\left(\mathrm{c} / \mathrm{m}_{\mathrm{e}} \mathrm{c}\right)^{2}\left(\mathrm{~m}_{\mathrm{e}} / \mathrm{M}_{i}\right) \mathrm{k}_{2}^{2} \mathrm{~A}_{\mathrm{k}_{0}}$. Since the electromagnetic wave frequency, $u_{0} ;$ is muli greater than the ion frequency, we can use the approximations,

$$
\begin{aligned}
& -\left(-\alpha_{0}+w_{2}\right)+k_{k}^{2}-2 i\left(-w_{0}+w_{2}\right) !_{1}=2 \omega_{0}\left(w_{k_{1}}-w_{0}+w_{2}+i i_{1}\right) \\
& a^{-\left(+w_{1}+w_{2}\right)+w_{k}^{2}-2 i\left(+w_{0}+w_{2}\right) T_{1}=2 \omega_{0}\left(\omega_{k_{1}}-w_{0}-\omega_{2}-i \Gamma_{1}\right)}
\end{aligned}
$$

to arrive at the disperston relation

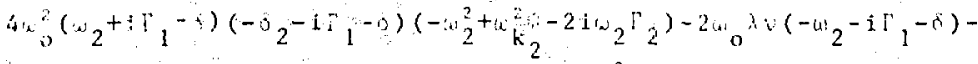

$$
\begin{aligned}
& \left.2 \omega_{0} \cdot 2^{2} \lambda v\left(w_{2}+i\right)_{1}-5\right)=0 \text {. }
\end{aligned}
$$


In this equation $\delta=\omega_{0}^{-\omega_{k}}{ }_{1}$. Eq (B8) can be rentitien in the more useful form

$$
\left(\omega_{2}^{2}-\left(\delta+\Gamma_{1}^{2}\right)+2 i \omega_{2} \Gamma_{1}\right)\left(\omega_{2}^{2}-\omega_{k_{2}}^{2}+2 i_{2} \Gamma_{2}\right)+\frac{\lambda v}{2 \omega_{0}}\left(\left(1-\alpha^{2}\right)\left(\omega_{2}+i_{1}\right)+(1+i) j=0\right.
$$

There are two cases of primary interest, the decay of the incident wave into normal modes of tho system( $\omega_{2}=x+i y s$, and a forced osciliation in which the secondary electromagnetic wave has the same frequency as the pump and the fiensity fluctuation is purely growing ( $\left.\omega_{2}=i y\right)$. The former case will be analyzed first.

Case $w_{2}=x+1 y$. First $l \in t$ us examine the instability atear threshoid. We expect the Erequency, $x$, to be approximately the normal mode of oscillation, the fon doustic wave. This suggests an expansion $x=\omega_{k_{2}}+\Delta$, such that $\Delta<<\omega_{k_{2}}$. It is convenient to intogduce the notation $2 B=\omega_{k_{2}}-\left(\delta^{2}+\Gamma_{1}^{2}\right)$ and $k=1 w$. ihen (59) separates into the following real and imaginary components,

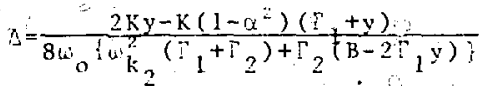

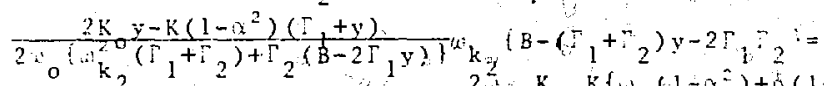

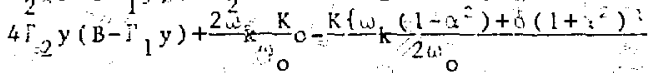

Working to frop order in $t$, we may eliminate for it as a function of $y$ ln (bla ) ond then substiating this value int $(B 10)$. We obtain:

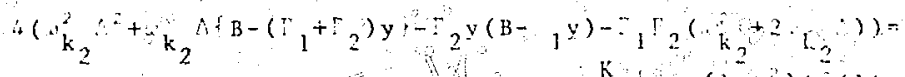

$$
\left.-\frac{k}{2 k_{0} k_{2}\left(1-a^{2}\right)+2(1+i)}\right)
$$

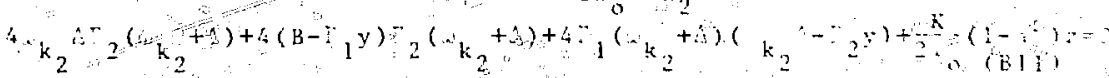
Thresholds may be dbatued by secting y=0 in (bi3). For a standing, wave pump $(x=1)$ a familiar coppession restits 


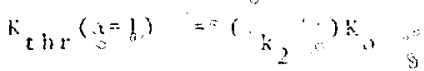

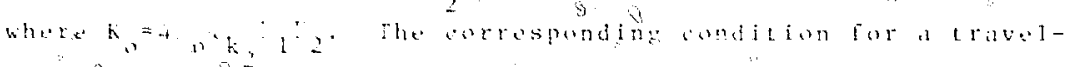

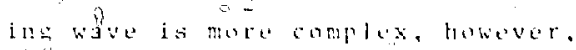

tat

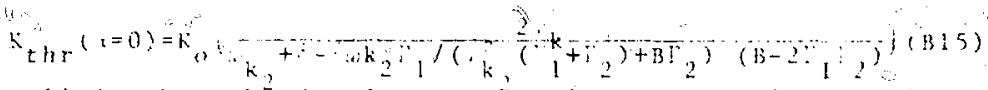

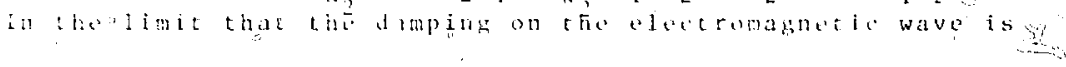

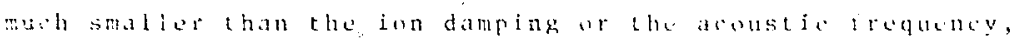

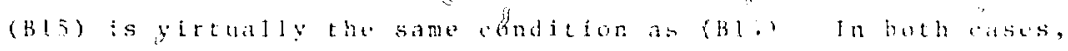

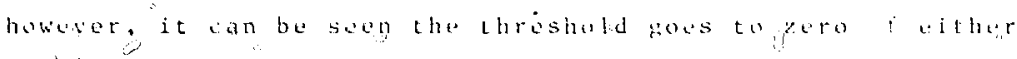

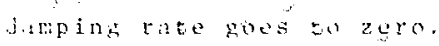

The mrowth rate near threshold is not a parameide wiolinterest in most cases, since it is by detinition closo longer.

Instead we will next alculate the growth rate far above threshold. By this we man that the damping rates are repligible

compared to the growth rates or the mismatch terms. Under those conditions. (B9) "reduces to

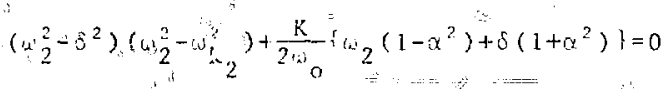

Again letting ${ }_{2}=x+i y$, the real and imaginary components are

$$
\begin{gathered}
\left(x^{2}-y^{2}\right)^{2}-\left(x^{2}-y^{2}\right)\left(\delta^{2}+w_{k}^{2}\right)+w_{2}^{2} \delta^{2}-4 x^{2} y^{2}+\frac{k}{2 \omega_{0}}\left\{x\left(1-x^{2}\right)+\delta\left(1+y^{2}\right):=0(B 17)\right. \\
2 x y\left(2\left(x^{2}-y^{2}\right)-\left(\delta^{2}+w_{k}^{2}\right)\right)+\frac{k}{2}\left(1-x^{2}\right) y=0
\end{gathered}
$$

These two equations can be combined to eliminate $\left(x^{2}-y^{2}\right)$,

so that (B17) becomes

$$
\left(x^{2}-y^{2}\right)=\frac{1}{2}\left(\delta^{2}+\omega_{k_{2}}^{2}\right)-\frac{K}{8 \omega_{0} x}\left(1-u^{2}\right)
$$

$-\frac{1}{4}\left(c^{2}+w_{2}^{2}\right)^{2}+\left(\frac{k}{8 w_{0} x}\left(1-\alpha^{2}\right)\right)^{2}-4 x^{2} y^{2}+\frac{k}{2 \omega_{0}}\left\{x\left(1-x^{2}\right)+\delta\left(1+\alpha^{2}\right)\right\}=0$

It is simple to solve (Bl9). Eor a standing wave, using the exDrassior for $\left(x^{2}-y^{2}\right)$

$$
y=\left(\frac{1}{2} \sqrt{a_{0}} k-\frac{\left(b^{2}+u^{2}\right.}{4} k \frac{1}{2}\right)^{\frac{1}{2}}
$$




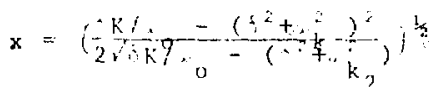

The maximum growth rate is found from $\frac{a y}{4}=0$ in (1320a). The maximum oceurs for $0_{0}=2^{-2 / 3}\left(\mathrm{~K} / \mathrm{\omega}_{0}\right) 1 / 3$, giving

$$
y_{\text {max } x}=\left(\frac{3}{8}\left(\frac{k^{2}}{2 \omega}\right)^{1 / 3}-\frac{1+k^{2}}{4} j^{1 / 2}\right. \text {. }
$$

Fur large pumperers, the growth rate far above chreshojd scales as $k^{1 / 3}$. These results agree with its kawa's equat ions, as chey should under these conditions.

Nishikawa did not consider the growth rate for a traveling wave punp. Eq (B19) then assumes the approximate form

$$
-\frac{1}{4}\left(i^{2}+z_{k}^{2}\right)^{2}+\frac{1}{2},\left(-\frac{k}{s_{1}}\right)^{2}-4 x^{2} y^{2}+\frac{k}{2 w_{0}}\left(k_{2}+k\right)=0
$$

This equation is quadratic in $x^{2}$, with the sciution

$$
x^{2}=\frac{k}{2 w_{0}}\left(\frac{(\omega}{2}+\frac{5)+\sqrt{(i n}+k)^{2}+y^{2}}{2}\right)
$$

There are two limits in which $x$ and $y$ are easily obtainable, (1) $y k_{2}+b$. and (2) yca $k_{2}+\dot{b}$. The latter has only limited since a large growth rate was assumed in deriving (B16). The strorg growth analysis, (1). then proceeds as follows.

Assuming $y>w_{k}+\hat{c}, \quad(B 22)$ becomes

$$
x^{2}=\frac{k}{16 w_{0} y} \text {. }
$$

which can thes be substituted in the earlier expression for $x^{2}-y^{2}$. The resulting solutions are

$$
\begin{aligned}
& y=\left(\frac{K}{\omega^{\prime}}\left\{\frac{2+\sqrt{3}}{16} !\right\}^{1 / 3}\right. \\
& \left.x=\left(\frac{K}{\omega_{0}}\left\{\frac{1}{16(2+3)}\right)^{1 / 2}\right\}\right)^{1 / 3}
\end{aligned}
$$

This solution possesses the same intensity dependence as the standing wave solutions, in the large growth linit, i e. as $\mathrm{K}^{1 / 3}$. The difference in the numerical factors is also small, so that wo may conclude that the instability whirh results from 
the coupling of ion density fluctuations with eletromenteltat waves is insensitive to the standing or traveling and natat of the pump, when the growe rates gruatly excesd the ind

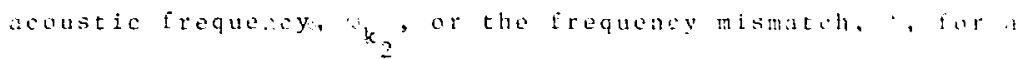
normal mode decas type interaction.

Case a (B9) becomes

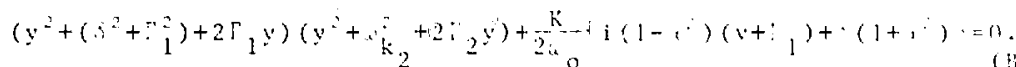
The requirement that $y$ is roal means that this type ot molution is possible only for $a=1$. Thus a purely growing instability can only occur if the pump is purely standing. An! traveling components will lead to a very small, hut initu, frequency component.

Threshold is obtained from (325) by settiris $y=0$,

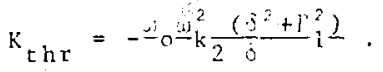

The threshold attains its minimum value at $i=-\ddot{\prime}_{1}$.

$$
\mathrm{k}_{\mathrm{min}}=2 \mathrm{w}_{0} \mathrm{ou}^{2} \mathrm{k}_{2} \Gamma_{1} \text {. }
$$

Note that (B26) is meaningful only for ico. Thereforc, a necessary condition for the purely growing instabilitv is that onlv waves such that $k \tilde{>} k_{\text {pump }}$ are unstable.

Far above threshald we expect the damping rates to has little effect on the instability. Neglecting r." " 2 . and "k2 with respect to y in (B25) gives

$$
\left.y=\left(\frac{-\delta^{2}+\left(\delta^{4}-4 \hat{n} \mathrm{~K} / \omega_{0}\right.}{2}\right)^{\frac{3}{2}}\right)^{\frac{2}{2}}
$$

For a stariding wave pump. The miximum growth rate occurs at $j_{0}=\left(-k / 2 \omega_{0}\right)^{1 / 3}$, 


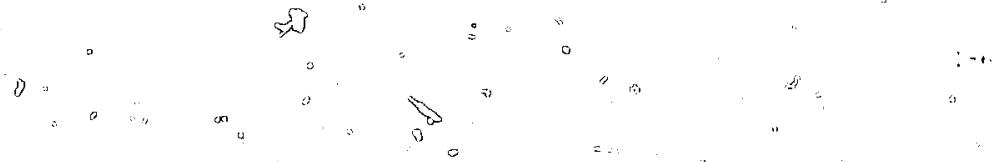

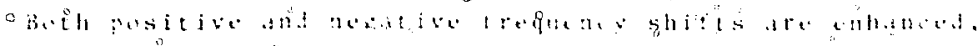

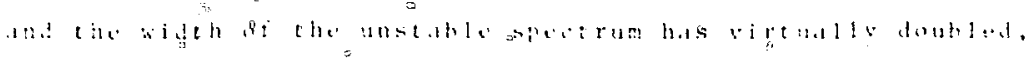

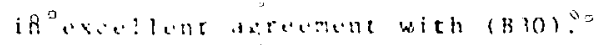

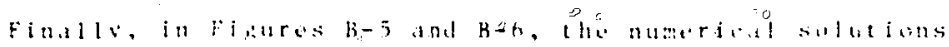

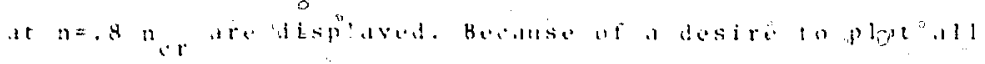

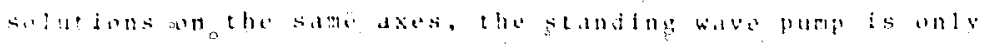

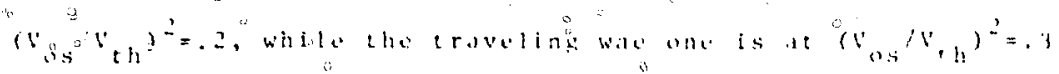

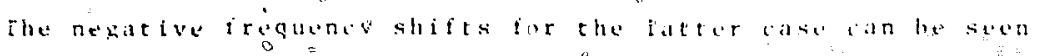

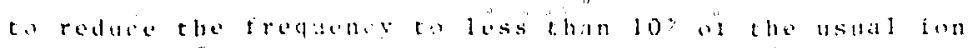

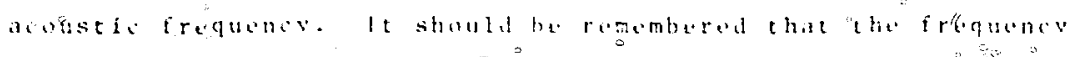

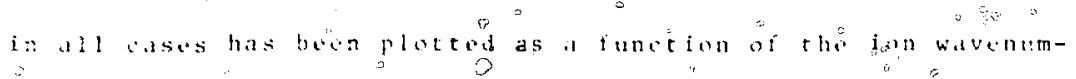

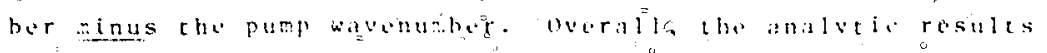

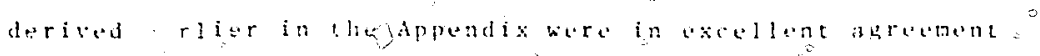
with the numerial solutions. 0
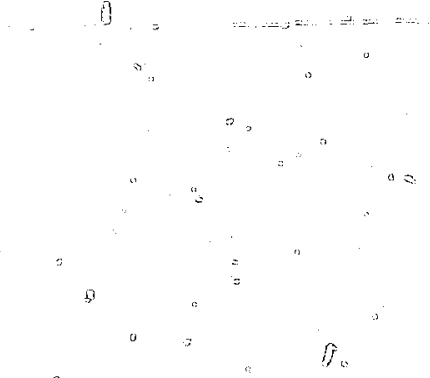

8

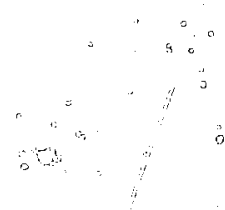


$\therefore 5$

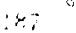

$3^{\circ}$

a

$\because 0$

y

a

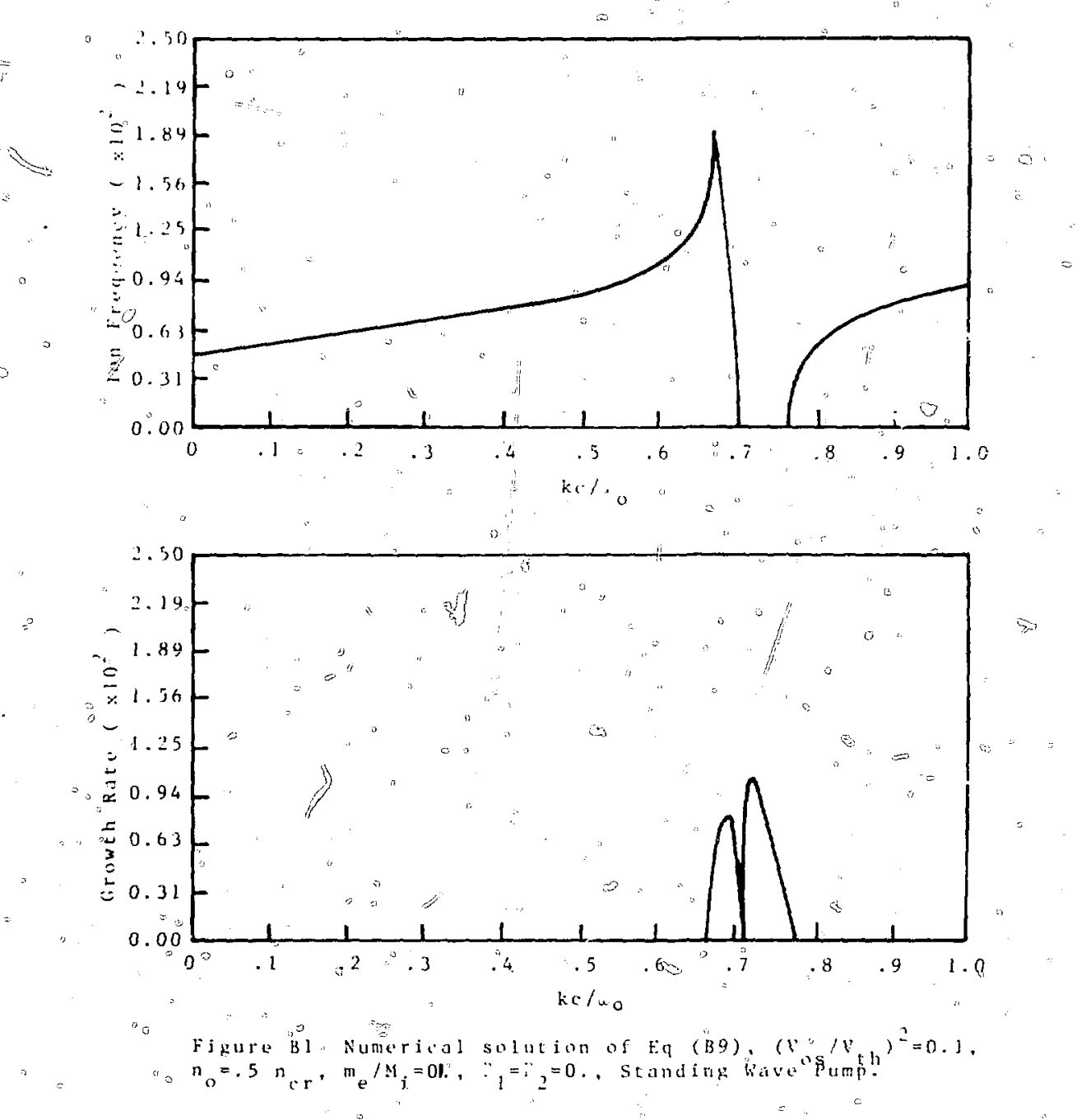




(3)

? $=0$

$=\quad . \quad 0$

अ-

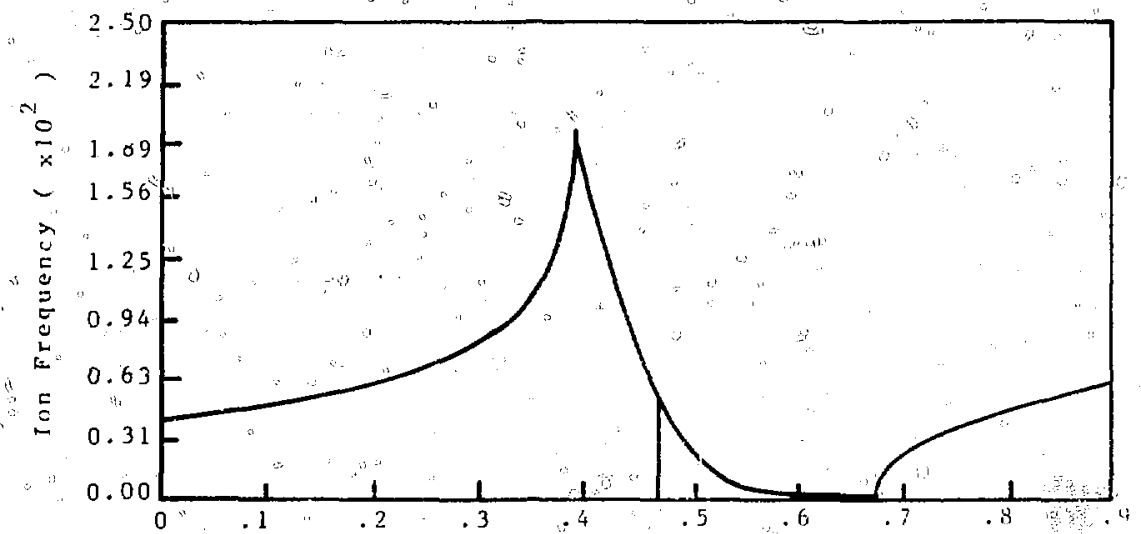

$k \cdot / \cdot \mathrm{a} a$

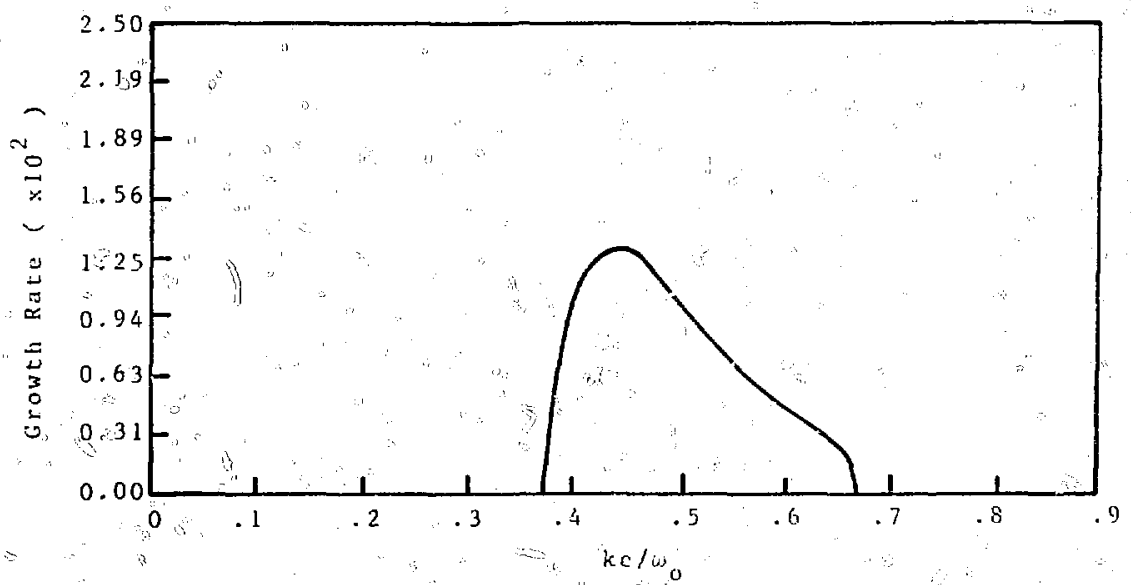

Figure BG Numerical solutions of Eq (B9), $\left(\mathrm{V}_{0 \mathrm{~s}} / \mathrm{V}_{\mathrm{th}}\right)^{2}=0.3$, $n_{0}=8 \mathrm{ncr}, \mathrm{m}_{\mathrm{g}} / \mathrm{M}_{\mathrm{i}}=01, \Gamma_{1}=\Gamma_{2}=0$. , Traveitan Wavespump. 
चा

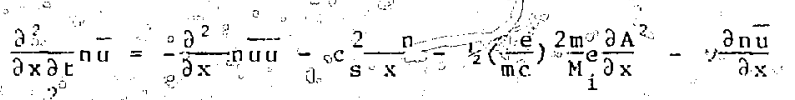

where $e_{s}^{2}=\left(\theta_{e}+\theta_{i}\right) / M_{i}$. Now, note, that.

$$
v \frac{\partial n \bar{u}}{\partial t}=v \frac{\partial n}{\partial t}
$$

and neglect convection in periodic systems on the grounds trat it is the radut ot two ejrot order quantities. Then (C4) and" (C5) lan be copoined co"give

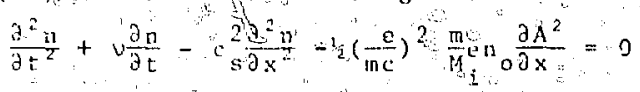

Fourier analysis" of (CG) "yieids

$$
\frac{\partial^{2} n}{\partial t^{2} k}+v \frac{\partial n}{\partial t}+c^{2} k^{2} n_{k}=-2\left(\frac{e}{m c}\right)^{2} \frac{m_{k}}{M_{i}} n_{c r} k^{2} 5 A_{k}, A_{k-k}
$$

For nodes, Equations $(C, 3)^{\circ}$ and (C 7$)$ represent $2 N$ coupled harmonic oscillators, where the coupling functions are derived aituraliy in the process of dering the equations. Similar sets can oc derived to describe the coupling of any two types of waves.

$y+1$ 20 


\section{APFFNDIX D}

Dezivation uf the Ion Factualion lnduced bissipation Rate

A simple, heuristic derivation can be siven for the is:ssipation rate whith ion tluctualions induce in the presence of a high frequency wave. (This follows a derivation miven by lirur..) Consider a density fuctuation of wavenumber, . magnitude. Ba, and a frequeney is much less than that of the high rrequenoy fiold. so that the lattor sees d constant perturbation over any periods. A high frequency electric field causes the inor. mobile electrons to translate with respect to the ions. If - the fiejd (electromagnetis or electrostatic) has the form

$$
\mathrm{E}=\mathrm{E}_{0} \cos \left(\mathrm{s}_{0} t\right)
$$

then the electrons will coliecrively exhibi- the valocity

$$
v_{O S}=-\frac{2 E}{m\{t} \operatorname{osin}\left(u_{0} r\right) \text {. }
$$

This gives an oscillating current.

$$
J=-e n v_{D S} \text {. }
$$

Since the electric field is presumed to be uniform in the rield direction, the continujty equation tor the sectrons . ir.is

$$
-i x)_{0} \delta n(\bar{x}, t)=-\bar{v}_{0 s} \cdot \nabla_{u}(\bar{x}, t)
$$

The field in the plasma generated by this oseillation o. . . is

$$
\nabla \varepsilon \bar{E}=4 \text { ire } \delta \text {. }
$$

Harmonic analysis of (D2) gives

$$
\mathrm{E}\left(k, \omega_{0}\right)=-4 \pi \mathrm{ei} \delta_{n}\left(k, \omega_{0}\right) i s\left(k, \omega_{0}\right) \text {. }
$$

The rate at wich these internal. ficlds do work per unt volume 
is just

$$
\frac{d L}{d t}=-\bar{J} \cdot \bar{E}
$$

How denote the rourier transtorm or sn by snk,

$$
\Sigma_{\mathrm{i}}=\sin _{k} \mathrm{e}^{i \bar{k} \bar{x}} \text {. }
$$

so that ( $(04)$ may be written

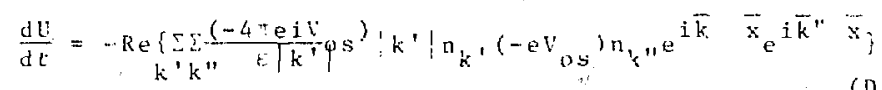

7.f the density fluctuations, $n_{k}$, are normalized with respect

to $\mathrm{n}_{\mathrm{cr}}$, and it is noted that $\mathrm{a}_{0}^{2}=4 \pi 0^{?} \mathrm{n}_{\mathrm{cr}} / \mathrm{m}_{\mathrm{e}}$, (D5) can be Ereatly simplified.

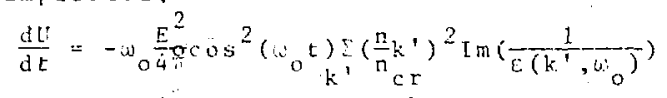

For an eIectrostatic wave, note chat

$$
\frac{d U}{d t}=-\nu^{*} \frac{E^{2}}{8}
$$

Comparison ot (DG) and (107), therefore gives a time averaged dissipation rate of

$$
v^{\prime \prime}=-w_{0} \sum_{k^{\prime}}\left(\frac{n_{k}}{r_{c}}\right)^{2} \operatorname{lm}\left(\varepsilon\left(k^{\prime}, \omega_{0}\right)\right) / \varepsilon^{2}\left(k^{\prime}, u_{0}\right)
$$

For a three dimensional distribution of density factuations, on iy the component aligned with the electric field contributes to (DB), so the expression within th? slimend should rigorously De multiplied ty $\cos ^{2}\left(\bar{k}^{\prime}, e-\bar{E}_{?}\right.$.

The dissipacion rate derived above is for the induced plasma fields, but since. their saurce is the electromagnetic field, (D8) also représents the electromagnetic damping rate as iong as $\nu^{*} \kappa_{\mathrm{k}} \omega_{\mathrm{p}}$ If the inequality is rot well satisied, depletion of the priasma fialds must be accounted for, and (DB) will be only an ifper bound. 


\title{
APJESDX E
}

\begin{abstract}
Application of I thomogenous Calculations to a Scattering Experiment Ferformed at SiRl.
\end{abstract}

The inherent three-dimensionality of SBS should not be obscured by the attention spent on the backscattering mode. This latter is interfating in itselt, since it has the lowesc threshold in a homogeneous plasma. For short dersity scale leng*hs, however, the threshosd for sidascater is lower thar for backscatter. According to Lia and Rosenbluth ${ }^{2}$, the inlomogeneous threshold for Brillouin backscatter is given oy

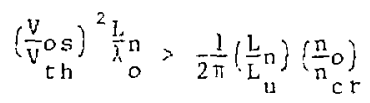

there $l_{n}\left(L_{u}\right)$ is the density (velocity) scalc length. Since the derivatives which ofitue the scale length are in the direction of propagation. oblique incidence increases the scale lengths and decreases the threshold. The same source 22 also derived a threshold condition for Brillouin sidescatter in a spherical plasma, radius of curvature, R, $\left(\frac{V}{v} \circ s\right){ }_{t h}^{2} x_{0}^{L}>\frac{1}{2 \pi\left(k_{o} l_{u}\right)^{1 / 3}\left(1+2\left(\frac{L}{R} n\right)\left(\frac{k}{k} y\right)^{2}\left(\frac{n}{n} c r\right)\left(1+\frac{L}{L} u\right)^{2 / 3}\right.}$

Under common experimental condilions, the sidescatter threshold is much lower than the backscatter. To illustrate this, we will consider parameters typjaal of a series of NRL experiments 15. In the NRL experiments, a subnanosecond 1.06 th laser whs focused onto a slab target. Although the condensed account of the experiments suggested a varisty of interesting pieces of information, the primary point of interest here was that they 


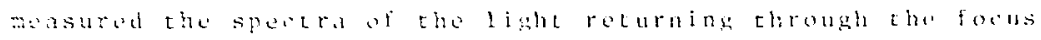
in: lens, Theso measurements were condected at three ditfureat oriantations of the tarect with respect to the laser. the angitale ot the signal collected by rhe lens was virLually independent at the orientation, althougi: a slighe increase with incrasing angle was avident in their publisided data. Sirec no stalisgies were given. it is not possible to infer treands from such small variations 15\%). Thu spectral sharacteristics, however, changed qualitatively as the orientation increasod from $0^{\circ}$ to $24^{\circ}$ io $45^{\circ}$. The spectra whibiced progressibely more struccure, and at $45^{\circ}$ a welidefined asvmetrical red-shift. The purpese of publishing the data was to support a Brilloujn haskscater hypethesis. lising the pubished data, however, and the results of the homogeneous periodic RDF caiculations, it is the intentill here to show that substantial sidescateer rould also have been measured.

The densiby and velocity acale lengths were theoretically estimated to be 30 and 200 b by Ripin. Evaluacing (FI) and (E2) at $n_{0}=.5 n_{e r}$ for normal laser incidencs gives $\left(v_{0 s} / v_{c h}\right)^{2} L / t=.012$ for backscatter threshold. but .002 for sidescatter threshold. For oblique incidence, both scale lengths are increased as $\mathrm{L}_{n}=\mathrm{L}_{\mathrm{n}} / \cos \theta$. The backscatter ihreshold decreases as a simple cosine of the incidence angle. The velocity gradient is in the ion acoustic direction, which is decermined by the wavenumber matching condition. The sidescacter threshold thus 


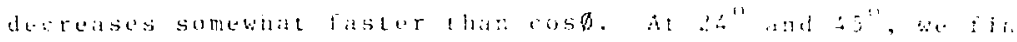

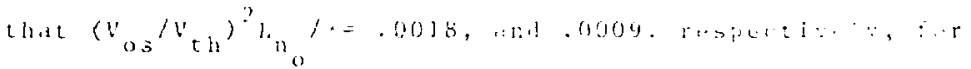

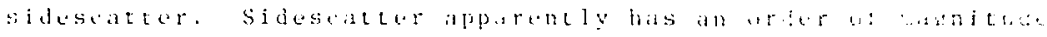

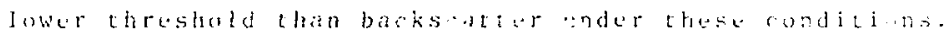

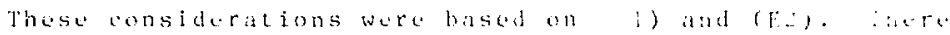
is no particular reason to dou, these eaprosions, bus thes are only threshold conditions. What is usually mure iateresting is the pump strugh at which a large fracting : the laser begins to be scatered. Particle simulations indicate that

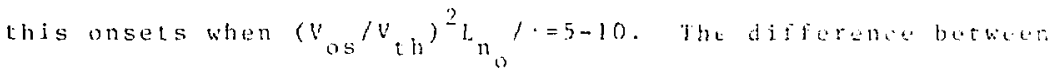
this supression and (El) is two to three orders or marnitude for backsoater. If one can extrapolate the difference fur sidescatter, then the threshold level providos an accurate estinate of the strength of the process. Confirmation of this for Brillouin sidescater or for the ettent of. Iistence ot

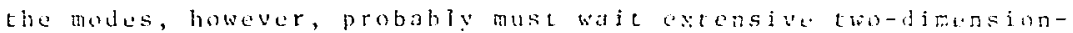
al namerifal simulations. Sidescater nevertheless secus highly competitive with batkscatter in the XRl experiants. Aarking, the hard evidene a mult

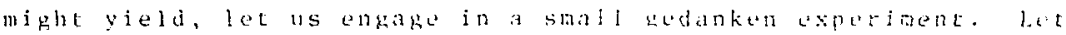
us assume that strong siascater ocour in the plasma. Since thero is no reason to think this would inbibit backsateter, ke may also assume that the rwo modes are sutideiently suparat

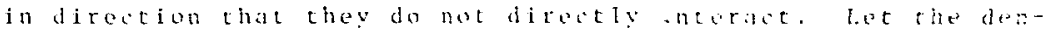

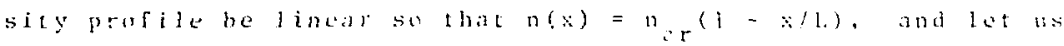

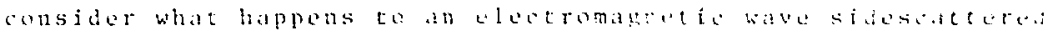




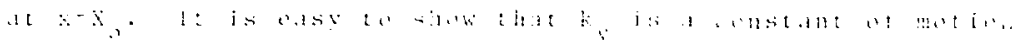

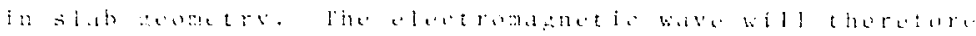

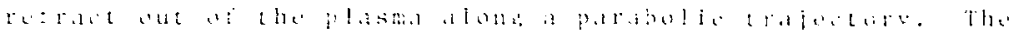

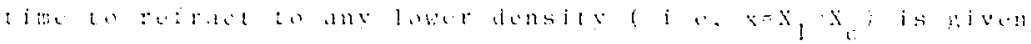
?

$$
\because\left(x_{1}-x_{1} ; 1\right)^{3}
$$

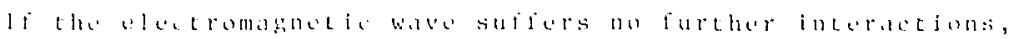

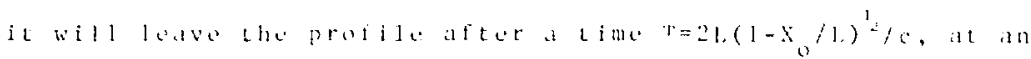
angl: $H=t_{\text {an }}{ }^{-1}\left(k_{y} / h_{x}\right)$, or

$$
\theta=\tan ^{-1}\left(\frac{x}{1-x_{0}}\right)
$$

A suff:oient]y intuse sidescatered wave illa undergo tureher interactions, howiver. There are too distinct factors which influence this. The first is the trajectory, which rs noted above will be approximately parabolic regardless of intnesity. Although this path is independent of the nature of the nen interations, it plays a vital role in determining the viability of wave-wave processes. For parametric processes sspecially, the trajectory determines the rate at which phase misinatich occurs. If we consider the stimulated backscatter instability for this wave, a coherence length, $L$, can thereTore interred,

$$
x_{0}^{L}\left(k_{0}-k_{1}-k_{2}\right) d x=1
$$

where $k_{l}$ is the wavcinumer of a backscatterdd wave, ko that of the refracing sidescattered wave, and $k_{2}$ that of an ion acoustic wave. Intorestingly. the small frequency shift in the secondary wave ma ths that it will retrace the path of the 


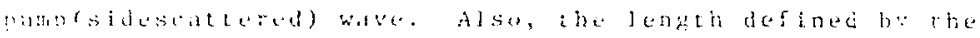

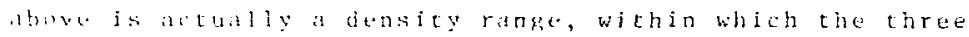
sive aroindierent.

The above integral can be explicitby evaltated for a lifuar density bratient. The y components are assuned a be be peractly resonat initially. In a slab geometry, therefore, cher will always be resonant. The evaluated integral gives

$$
L-x_{0}=1 \cdot\left(\frac{4 c}{\left.3 \omega_{0}\right)^{2 / 3}}\right.
$$

The tinc required for the wave to propagate to this lower density is then

$$
\Delta t=\frac{32}{3 u_{0}}\left(\frac{w_{0}}{c} \frac{L}{c}\right)^{2 / 3}
$$

Finally, the distance the wave will have traveled in the y-direction in this time is

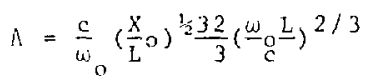

Tha interaction length for stimulated brillouin backscater is thus $L_{1}=\left(\Lambda^{2}+\left(L-X_{0}\right)^{2}\right)^{\frac{1}{2}}$.

Effective backsatter can only occur if the sidescietered intensity along the above interaction pach is sufficiently intense. Inhomogeneous simulations have shown that the product of $\left(\mathrm{V}_{\mathrm{os}} / \mathrm{V}_{\mathrm{th}}\right)^{2}$ and $\mathrm{L}_{\mathrm{n}}$ must exceed 5-10 for strong backscatter to occur. The scale jength there was the full density profile length, and not the interaction one. Taking the lower bound gives a threshold (for strong scatter) ( $\left.\mathrm{os}_{\mathrm{og}} / \mathrm{u}^{2}\right)^{2}=.25$ for the NRl experiments. The actual sidescattered intensity can be estimated only in the crudest fashion, but since its inhomogeneous threshold is an order of magnitude lower than 
backscatere, we expect at least a comparable anount of energy to be sidescatcerod. In the ikt experiment, the Eocused power lensity wis estimates to be $3 \times 10^{15} \mathrm{k} / \mathrm{em}^{2}$ and the cleseron temperacure to be $7 \mathrm{keV}$. This corcespouds to $\left(\mathrm{V}_{\text {os }} / \mathrm{S}_{\mathrm{th}}\right)^{2}=\dot{4}$. Stimulated reflection in the batkwial direction has been measuled to be abut $10 \%$ in many experiments, though mly $5 \%$ in the NRl experiment. Even this amount, though, lends credence to the supposition that the sidescatcered light in the NRL experiment wis intense enough to stimulate strong Brillowin backscater.

Linder the above conditions, strong SBS would be similar to the model mode coupling calculations discussed in Chapter 3, Section 1. Repeated scattering is therefore probable. In fact, if the intensity of the sidescatcering could be determined accurately, those results could plausibly extrapolated to give the magnitude of the spectral broadening. As it is, cialculations at $n_{0}=.5 n_{\mathrm{cr}}$ with modest pump levels led to $8 \%$ relative bandwidth. When this is scaled down to ralistic mass ratios, a bandwidth of $.5 \%$ is still obtained. This compares well with the bandwidths reported in several scattering experiments. infortunately, quantitative comparisons with the repented scattering calculations will be impractical until the magnitude of sidescaltering can be nore accurately gauged.

Findly, the sidescattering hypothes is can reasonably accout for increasing spectral width with increasingly oblique targec irradiation. Ii we supoose that the expansion plume dynamics are dominated by the shab surfare, then the oblique incidence may decually correspond to the incidence angle relative to the plasina normal surface. The scattered spectra were measured from light collected by the foowsing lens, either

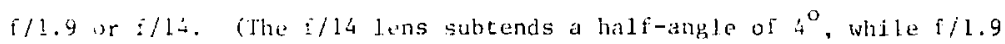


subtends almost $30^{\circ}$.) for nornal incidence, a nar row backscatered signil was measured, which is entirely consistent with stimulated Brilluoin backscatter. Even with the t/.9 lens, the original sidescaterIng event would have been forced to occur above about $n_{0}=8 \mathrm{n} \mathrm{cr}(.995$ ner with the filt lens.). AL $24^{\circ}$ ineidence, the total back-signal was only slightly grtater, some oroadening was cuident, mu the signal was still doninated by the narrow contral peak, here, the $1 / 14$ lens could collect sidescatter originated between . $8-.9 \mathrm{n}_{\mathrm{cr}}$, which sems too high a density for strong sidescatter. If the other lens were used, nowever, densities as low as . 5 ner could have been sampled. This certainly inciudes part of the density regime in which sidescuttering might be expected. The IRL report did not specify which lens was used for these measurements, unfortunately, so the incermediate angle must be regarded is ambiguous for these purposes.

The broadest, most diffuse spectra were measured iron targets tilted $45^{\circ}$ to the laser, but with total energy comparable to the others. Light refracted from between $n_{0}=.4-.6 \mathrm{n} \mathrm{cr}$ would have been collected by the f/lt lens here, and to paraphrase coldilocks, this lits (sidescattering) just (about) right. The reasoning behind this is straightlorward. Scattering processes occurring above $.8{ }^{\prime \prime}$ are doubly weakened because the ion wavelength is increasing, and so decreasing the growth rate, and because the process must compete with a robust ensembie of absorptive mechanisms near the critical densicy. Below about. 25 $\mathrm{n}_{\mathrm{cr}}$ on the other hand, Bailouin scattering thust compete with the stronger Raman back- and sidescattering processes. The logical regime to look for Brillouin scatcering is thus between .3 and $.7 \mathrm{n}$. Ine Eact that the best defined examples ot spectr: 
broadening eccurred at $4^{\circ}$ strongly suggests that sbs was involved, while the inhomgeneous simulations in Ch 3 , Section Il showed that backsvattering was too weak a broadening source to account for the measurements. For a variety of reasons then, Stimulated Brillouin sidescatter, followed by repeated backscattering, must he considered as consistent with the NRL experiments, and at least as attractive as any other broadening mechanism for these conditions. 


\section{APPENDIX F \\ Derivation of Jarnbean have Function}

Equation $(4-5)$ was the nonlinear wave "quation derived in Chapter 4. If a stauding wave is assumed, and the exponential nonlinearity in $(4-5)$ expanded for weak fields, i e, ro $\geq 1$, the following equation results

$$
\frac{a^{2} \mathrm{~A}}{\partial\left(x k_{0}\right)^{2}}+\left(1-a+l_{2} n_{0}^{2} A^{2} a\right) A=0
$$

where $a=2\left(n_{0} / n_{c r}\right) /\left(1+\exp \left(-\frac{1}{2} n_{0}^{2} \Lambda_{\text {max }}^{2}\right)\right.$. If we let $A=c_{1} c n\left(x k_{0}, m\right)$. (Fl) becomes

$c_{1} c n\left(x k_{0}, m\right)\left(2 m-3-2 m c n^{2}\left(x k_{0}, m\right)\right)+\left(1-a+\frac{1}{2} n_{0}^{2} a C_{1}^{2} c n^{2}\left(x k_{0}, n\right)\right) C_{1} c n\left(x k_{q}, m\right)=0$

Eq (F2) is satisfied for two choices for $\mathrm{m}$

$$
\begin{aligned}
& m=a / 2 \\
& m=m_{0}^{2} c_{1}^{2} / 4
\end{aligned}
$$

If the first expression is chosen, identicai satisfaction of (F2) occurs only at $n_{0}^{2}=2 / C_{1}^{2}$, which is also the matching point for the second choice. Since a standing wave always gives $C_{1}^{2} \geq 2$. the exact matching is at least in fair agreement with the assuaption of weak fields, to expand the exponential nonlinearity. The jacobean function, cn, therefore is a solution to the cubic nonlinearity wave equacion only at one field strength, but we expect it to be close to the exact solution for a spread of field strengths around that point. 


$$
\text { Cr: '.'TR } 4
$$

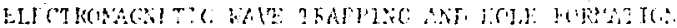

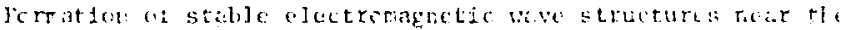

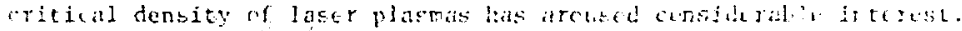

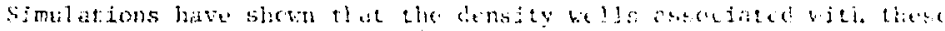

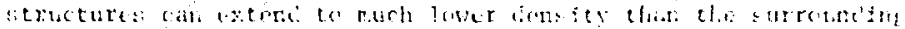

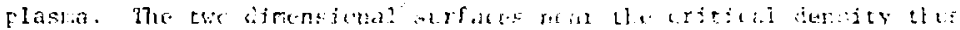

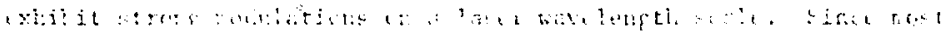

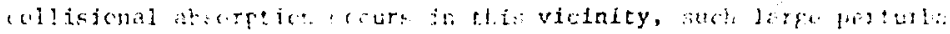

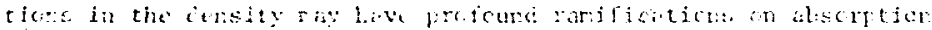

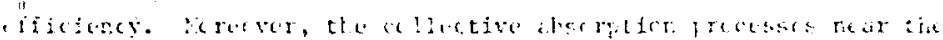

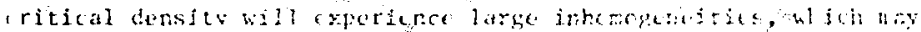

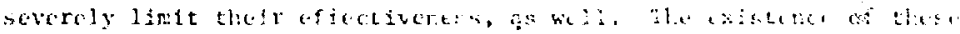

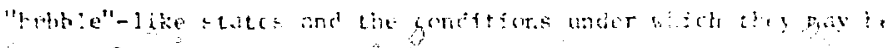

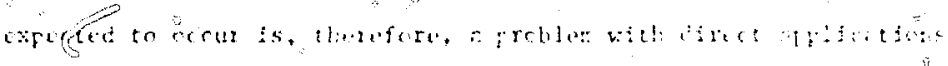

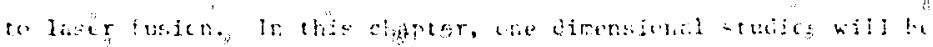

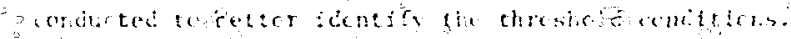

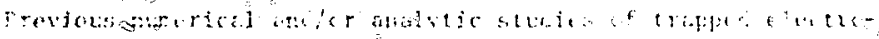

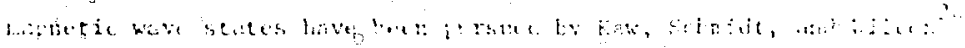

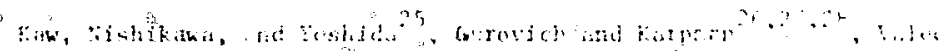

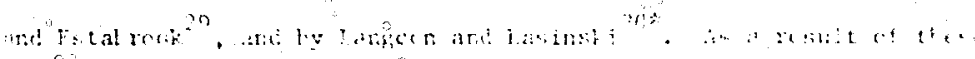

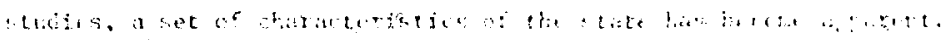

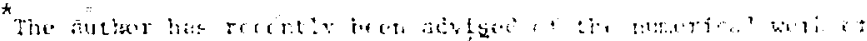

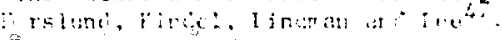


18. F. Shimizu, phys. Rev. Leters 19, $10971996 \%$.

19. J.J.thomson, R.l. Faehl, and W. L.Kruer, Phys. Rev. Leters 31 , $918(1973)$, J.A.thomson, R.J.Faeh], W.L.Kruer, and S.E. Eodnter, Phys. Fluids 1i, $973(1974)$.

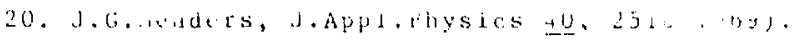

21. W. L.Kruer, Advences in Plasma physics, tol 6, ed. A.Sinon and W. B. Thompsan. (.john h'i ley sons, New iork, 1976). 237.

22. C.s.liu, Marshall N. Rosenbluth, and Roscoe R. Whita. thys.

Fluits 17, 1211(1974), C.S.iu, Aivances in Plasma Physics, Vol 6 , ed. A.Simon and K. B. Thompson, CJohn hiley s sons, Sew Fork, 1976), 121 .

23. W.L.Kruer, E.J.Valeo, and K.G. Estabrook, Phys. Rev. Letters 35. $1076(1975)$.

24. P.Kaw, C.Sohnidt, and T.Wicox, Phys, Fluids 16, 1522(1973).

25. P.kaw, K. Nishikawa, and Foloshida, Phys. Rev. Letters 35 . $88(1975)$.

26. V.Ts.Gurovich. V.I.Karpman, and R.X.Kaufman, Sov. Phys. JETP $\underline{29}, 1063(1969)$.

27. V.Ts.Grovich and V.J.karpman, Sov. Phys. JeTP 30, 798(1970).

28. V.Ts.Gurovich and V.I.karpman, Sov. Phys. JETP 29, $1048(1969)$.

29. E.J.Valeo and K.G.Estabrook, Phys. Rev. Letters 34, 1008(107:).

30. A.B.Langdon and B.F.Lasinski, Bu11. Am. Fhys. Soc. 19, $920(1974)$.

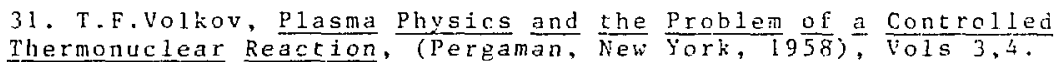
32. J.H.Marburger and R.F.Tooper, Phys. Rev. Letters 35, I001 (1975).

33. C.S.Lai. Phys. Rev. Letters 36, $966(1976)$.

34. V.E.zakharov, Sov.Phys. JETP 35, 908(1972).

35. E.J.vales and H.L.kruer, Phys. Rev. Letters 33,740(1974).

36. J.Denavit, N.R.Pereita, and R.N.Sudan, Phys. Rev. Letcers 33, $1435(1974)$. 
3.. R.h.Harvey and George Schmidt, Phys. Fulds 18, 1395(1975).

H.C.Kim, R.L.Stenzel, and A.Y.Wong, Phys. Rev. Letters 33 , S6́t(i575). R.L.stenze1, A.Y.Wong, and H.C.Kim. Phys. Rev.

$\operatorname{lot} \operatorname{tans} 32,654(1573)$.

39. K. Eidmann and R.Sigel. Phys. Rev. Letcers 34, $799(1975)$.

-0. I.P.Donaldson and I.J.5palding, Phys. Rev. Letters 36. 467 $(1976)$

4. D.W.Forslund, J.M.Kindel, K. Lee, and E.L.Linuman, Phys. Rev. Letters $36,35(1976)$.

A2. 1.F.Seely and E.G.Harris, Phys. Rev. A I, 1064 (1973).

3. J.F. Seely, Phys: Rev. A 10. 1863(1974):

+4. D. Mckinnis and M.V.Goldman, CU \#1012. June 1975(Submitered to Physical keview).

⒌ V.P.Silin, Soy: Phys. JETP 20, 1510(1965).

46. l.s.Gradsiteyn and I.M.Ryzhik, Table of I jegrals, Series, and Products. (Academic Press, New York, 1965).

no klee, D.W.Forslund, J.M.Kindei, and E.L.Lindman, Bull. Am. pits. sice 2 l, $1067(1976)$.

48. I. Dawson and C.oberman, Plys. Fluids 5, $517(1962)$.

49 . C.oberman, Arkon, and J.Dawson, Phys, Fluids $5,1514(1962)$.

$500^{3}$. Dawson and C.Obetman, Phys. Fluids 6, 394 (1963).

51. D.CHontgonery and D.A.Tidman, Plasma Kinetic Theory,

(NoGraw-Hil1, 1nc., New York, 1964), 32 .

52. A.Y.Hong and R.L.Stenzel, Phys. Rev. Leters $34,727(1975)$.

53. Rubert D. Richtmyer and K.W.Morton, DifEerence Methods Eor Initial-Value problems, (Interscierce Publishers, New York, 1957 ).

54. K. Nishikawa... Phys.Soc, jap. 24, 916(1968). 